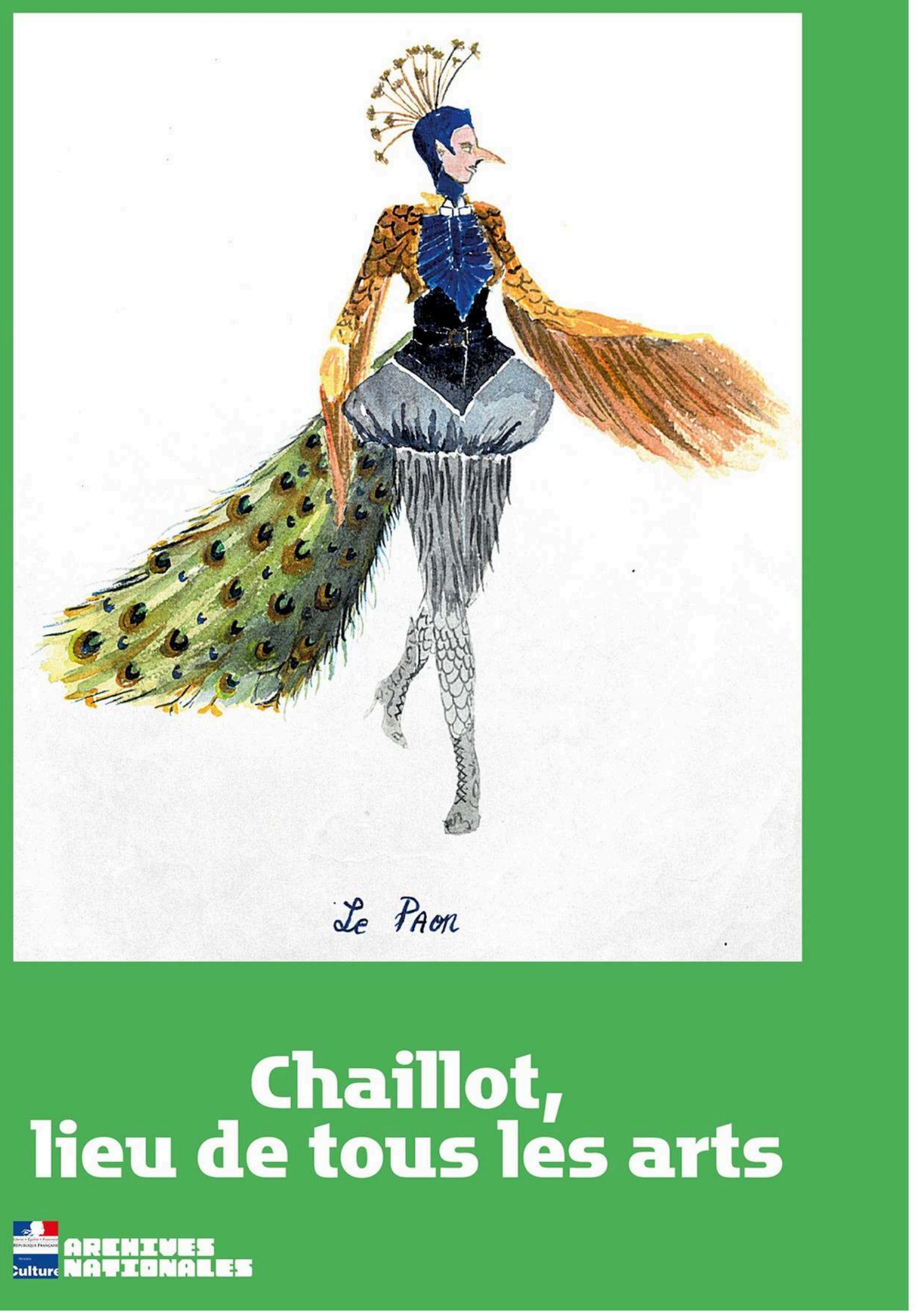




\section{Chaillot, lieu de tous les arts}

\section{Sandrine Gill (dir.)}

DOI : 10.4000/books.pan. 2185

Éditeur : Publications des Archives nationales

Lieu d'édition : Pierrefitte-sur-Seine

Année d'édition : 2020

Date de mise en ligne : 20 avril 2020

Collection : Actes

EAN électronique : 9791036558375

\section{(2) OpenEdition \\ Books}

https://books.openedition.org

\section{Référence électronique}

GILL, Sandrine (dir.). Chaillot, lieu de tous les arts. Nouvelle édition [en ligne]. Pierrefitte-sur-Seine:

Publications des Archives nationales, 2020 (généré le 20 juin 2022). Disponible sur Internet : <http:// books.openedition.org/pan/2185>. ISBN : 9791036558375. DOI : https://doi.org/10.4000/books.pan. 2185.

Ce document a été généré automatiquement le 20 juin 2022.

(C) Publications des Archives nationales, 2020

Conditions d'utilisation :

http://www.openedition.org/6540 


\section{RÉSUMÉS}

Dans le contexte des commémorations des 70 ans de la signature de la Déclaration universelle des droits de l'homme à Chaillot, les journées d'étude du 14 et 15 juin 2018 étaient consacrées à Chaillot, lieu de tous les arts, en partenariat avec Chaillot - Théâtre national de la Danse, la Bibliothèque nationale de France, l'ANR ECHO "[ECrire l'Histoire de l'Oral], les Archives nationales et la mission des archives du Service interministériel des Archives de France, placée auprès du ministère de la Culture. La première journée, aux Archives nationales, était consacrée aux sources archivistiques et à leur exploitation. La seconde journée, à la Bibliothèque nationale de France présentait les diverses facettes du Palais des arts du spectacle : de l'architecture à la musique, de la danse aux perceptions du public...

Des chercheurs, archivistes et étudiants en Études théâtrales mettent ici en lumière les fonds d'archives relatifs au Théâtre national de Chaillot conservés dans différentes institutions partenaires. Aux Archives nationales, les récents versements, exceptionnels, et inédits pour un théâtre national, d'archives audiovisuelles et sonores viennent compléter les archives papier versées par le théâtre dès les années 1960.

Enfin le témoignage oral de Nicolle Daviot, habilleuse au Théâtre national de Chaillot pendant plus de 50 ans, enregistrement réalisé par Sandrine Gill en septembre 2018, est désormais librement consultable aux Archives nationales (versement n²0200119).

\section{SANDRINE GILL (DIR.)}

Cheffe de projet archivage audiovisuel, département des Archives électroniques et des Archives audiovisuelles [DAEAA], Archives nationales 
SOMMAIRE

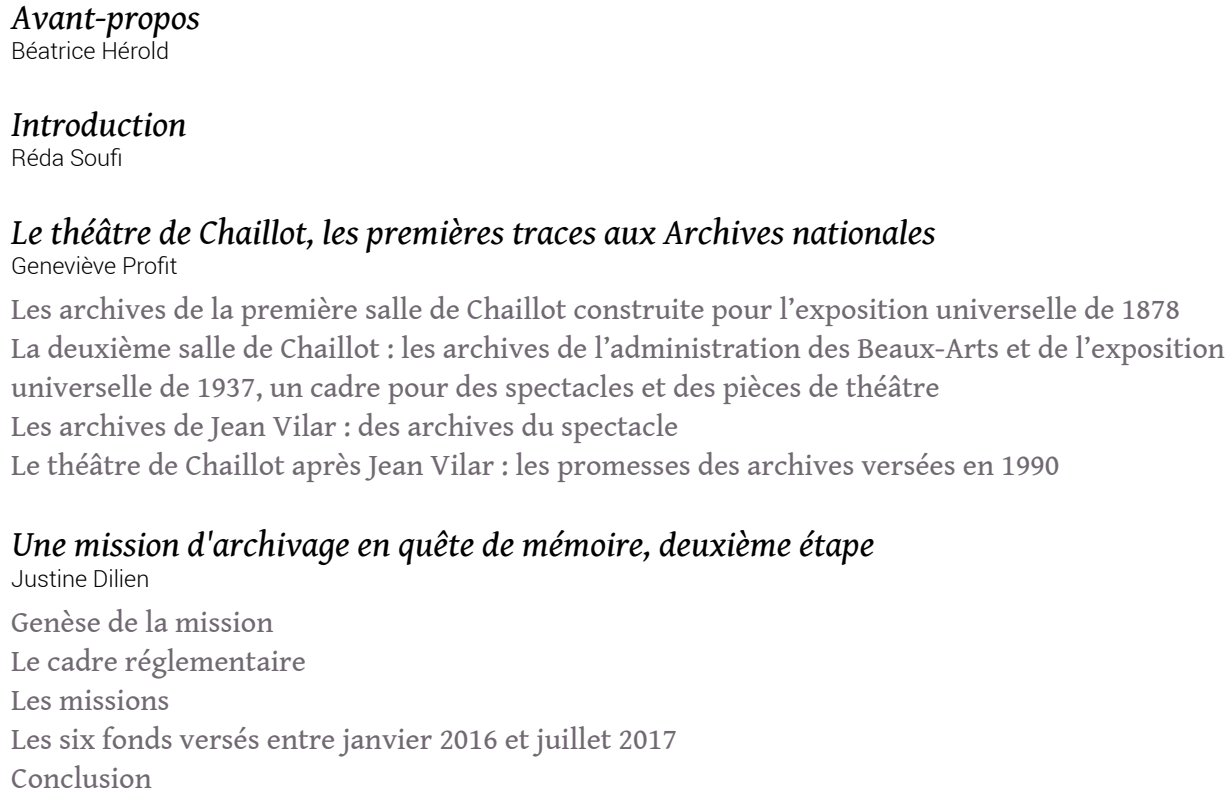

Une mission d'archivage en quête de mémoire, deuxième étape Justine Dilien

Genèse de la mission

Le cadre réglementaire

Les missions

Les six fonds versés entre janvier 2016 et juillet 2017

Conclusion

La mémoire des spectacles du Trocadéro et de Chaillot au département des Arts du spectacle de la Bibliothèque nationale de France

Joël Huthwohl

Le temps du Trocadéro

Le Théâtre national de Chaillot : une documentation et une iconographie abondantes

Les fonds d'archives

De la bobine au fichier numérique, une archéologie des archives audiovisuelles et sonores $d u$ Théâtre national de Chaillot

Sandrine Gill

Entrer au Théâtre national de Chaillot en poussant la porte des Archives nationales

L'archiviste, archéologue au chevet des archives audiovisuelles et sonores

Analyser le processus de création d'archives audiovisuelles, alternative à la démarche du metteur en scène

Complémentarité des modes d'archivage modernes du spectacle théâtral : archives sonores/ archives papier/archives audiovisuelles

Marie-Madeleine Mervant-Roux

Complémentarité des archives audio et des archives papier : reconstituer la genèse du Lorenzaccio discographique de 1956

Complémentarité des archives sonores et des archives audiovisuelles : comment rendre compte de Par les villages?

Cyrano de Bergerac, mis en scène par Jérôme Savary : le bruit du panache

Océane Djabellah-Peillon

Sonorités de la censure dans le Macbeth de Matthias Langhoff

Eva Bleibtreu

L'esthétique de Matthias Langhoff

L'archive étudiée

Démarche adoptée pour l'étude sonore de cette archive audiovisuelle

Observations découlant de la première écoute

Analyse d'un extrait de la mise en scène par l'écoute

Conclusion 
Les Aventures du petit père lapin : Les signes et les sons

Évangélia Pruvot

Les sons : musique, bruitages, voix

L'expérience ECHO

Les archives d'architecture : comprendre ce que fut l'outil du TNP à Chaillot Sandrine Dubouilh

La salle des fêtes de 1878 au prisme des ambitions du théâtre populaire

Le premier Théâtre National Populaire dans ses murs ; les comptes rendus d'exploitation

Un autre regard sur la genèse de la salle de 1937 par l'étude des archives d'architecture

Le TNP de 1952, héritier d'un cahier des charges obsolète

Le scénographe Jacques Le Marquet et le Palais de Chaillot

Anaïs Dupuy-Olivier

I. Jacques Le Marquet, régisseur au TNP

II. La salle Gémier (1963-1967)

III. Les projets de refonte de la grande salle (1963-1972)

Jeanne d'Arc au bûcher au Palais de Chaillot de 1939 à 1942 : une simple reprise?

Pascal Lécroart

Le contexte de la création parisienne au Palais de Chaillot

La reprise à Chaillot en 1942

Le programme de 1942

La réception dans la presse

Aller à Chaillot : des spectateurs d'aujourd'hui parlent et se souviennent Hélène Bouvier

Un lieu et une architecture, une empreinte et une emprise

Direction, troupe, répertoire et scénographie : une cohérence perçue et remémorée

Les effets conjugués de l'accueil du public et de la programmation du TNP

Une expérience personnelle indélébile

Conclusion

Béjart à Chaillot : une anti-histoire de la danse

Guillaume Sintès

Débriefer l'assemblée théâtrale

Daniel Deshays

L'utopie d'une cité du tout-théâtre

Brigitte Joinnault

Trois fois Chaillot

Réflexions sur le projet de cité du théâtre

L'arrivée à Chaillot, un changement d'échelle

Le tout-théâtre 
Avant-propos

Béatrice Hérold 
1 Les journées d'étude "Chaillot, lieu de tous les arts» ont constitué le lieu d'une confrontation paradoxale entre l'éphémère et la trace : l'instant de la représentation théâtrale, qui est le moment singulier d'une rencontre unique entre une œuvre, une troupe, un public, peut-il faire l'objet d'un archivage? La question est ouverte et les réponses plurielles, certains metteurs en scène poussant la logique jusqu'à refuser le filmage des spectacles. C'est néanmoins sur l'initiative et la volonté du fondateur du Théâtre national populaire, Jean Vilar, que les premiers versements de documents en provenance de cette institution vers les Archives nationales ont été effectués en 1966 : il souhaitait voir conservée la mémoire de l'expérience nouvelle qu'il avait conduite depuis la création du TNP en 1951.

Ces rencontres ont été l'occasion également pour les Archives nationales de se décaler de leurs perspectives habituelles et de tendre l'oreille au lieu d'ouvrir les yeux pour mieux saisir une catégorie encore peu exploitée, leurs archives sonores et audiovisuelles. Les travaux menés par les étudiants du master d'Études théâtrales de l'université Sorbonne Nouvelle-Paris 3 sous la conduite remarquable de MarieMadeleine Mervant-Roux, responsable du projet ANR ECHO, invitent en particulier à une formidable leçon sur la dimension sonore et auditive du théâtre : qu'est-ce qu'un document sonore sans visuel? Mais, a contrario, surgit la question suivante, insolite mais si logique, qu'est-ce qu'une image, prise sur le vif d'une scène, dénuée de son? Sans les voix, la musique et les bruits - les pas, les bruitages, les réactions du public ? Le son recèle une puissance évocatrice inattendue et riche d'enseignements et, nouveau paradoxe, il se révèle créateur d'images dans l'esprit de celui qui l'entend.

3 Les différentes contributions réunies ici sont donc pour les Archives nationales un puissant stimulant pour poursuivre le patient travail, parfois archéologique, d'identification et de contextualisation des archives sonores et audiovisuelles, qui sont souvent peu documentées lors des versements. À cet égard, la mise au point méthodologique de Sandrine Gill, cheffe de projet sur l'archivage audiovisuel, est particulièrement novatrice et éclairante. Il convient ici de la remercier pour cela et tout autant pour avoir été l'infatigable coordinatrice de ces journées et de la publication des présents actes.

\section{AUTEUR}

\section{BÉATRICE HÉROLD}

Directrice de l'Appui scientifique aux Archives nationales 


\title{
Introduction
}

\author{
Réda Soufi
}

1 Du Théâtre national populaire au Théâtre national de la Danse, Chaillot s'est toujours pensé comme un lieu unique, celui de toutes les utopies, de tous les possibles, celui de tous les arts.

2 Mémoire des lieux et des espaces, des hommes et des femmes qui y ont travaillé, du public qui s'y est pressé, tous, chacun à leur façon, ont contribué à construire la légende de Chaillot.

3 Alors que nous venons de célébrer le $70^{\mathrm{e}}$ anniversaire de la Déclaration universelle des droits de l'homme proclamée par les Nations Unies dans la grande salle du théâtre le 10 décembre 1948, c'est une autre histoire que nous sommes ici invités à découvrir, à esquisser : celle d'un théâtre qui, dès son ouverture en 1939, a marqué le paysage culturel français par la monumentalité de son architecture, l'ambition politique de son projet, la modernité et la contemporanéité de son identité artistique.

4 Cette histoire, il est possible de la restituer dans toute sa richesse et sa diversité à travers les nombreux fonds d'archives conservés principalement aux Archives nationales et à la Bibliothèque nationale de France et versés par les directions successives du théâtre pour lesquelles la question mémorielle a été une préoccupation constante depuis plus de 50 ans.

5 Programmes de salle, brochures de saison, dessins, croquis, plans, maquettes de décors, photographies, conduites de régie, captations audiovisuelles, bandes son, courriers, notes, registres des recettes de spectacles, contrats, conventions collectives, costumes, mais aussi paroles d'anciens spectateurs ou de comédiens collectées dans le cadre du programme de recherche ECHO de la BnF sur l'oralité de la mémoire à travers les témoignages, tous ces éléments constituent une véritable mine d'or pour la recherche, mais aussi pour le public curieux de découvrir de l'intérieur le fonctionnement d'une « maison » de spectacle.

6 Au-delà des objets, des témoignages, les archives de Chaillot prises dans leur globalité mettent surtout en lumière la rémanence de problématiques dans lesquelles se fondent et se confondent les préoccupations de gestion quotidienne et le déploiement d'un projet artistique au service de la démocratisation culturelle. 
7 La difficulté des conditions de travail est souvent pointée dans les récits de celles et ceux qui ont vécu et pratiqué Chaillot au quotidien, avec son lot d'espaces mal chauffés, mal aérés et tellement peu fonctionnels au regard des nécessités du métier.

8 Mais les archives de Chaillot illustrent avant tout les enjeux, débats, positions politiques et arbitrages qui ont progressivement façonné le lieu.

9 Ainsi en va-t-il des premiers travaux et plans d'aménagement de Jacques Le Marquet, régisseur à Chaillot dans les années 50 et 60 , qui déboucheront sur la construction de la première salle Gémier en 1966; de l'utopie de la cité du théâtre d'Antoine Vitez gouvernée par « un conseil des ministres » et pensée en termes d'espace, de langage, d'organisation du travail, ou encore du projet d'installer à la fin des années 70 l'école Mudra de Maurice Béjart dans le palais.

10 Ce travail d'archivage a récemment été relancé notamment pour répondre à la nécessité de vider de nombreux locaux et laisser place au vaste programme de travaux inscrit dans le nouveau schéma directeur de rénovation du théâtre qui doit aboutir, à l'horizon 2025, à une refonte complète de la salle Jean-Vilar après la restructuration de la salle Firmin-Gémier, achevée en 2017.

11 Dans ce contexte, grâce au soutien et au travail minutieux réalisé par la Mission des archives du ministère de la Culture, Chaillot a pu verser six fonds complémentaires de ceux déjà existants aux Archives nationales, notamment un fonds audiovisuel important composé de 576 vidéos numériques et 1821 supports analogiques et numériques.

12 Entre "tout garder" ou "tout jeter ", au-delà des obligations réglementaires et administratives, la généralisation de l'informatisation et de la numérisation, si elle offre des garanties solides pour la conservation et la préservation des documents, interroge nos pratiques en matière de transmission et d'archivage.

Elle permet, en tout cas, une plus grande circulation des documents et donc leur présentation au public comme en atteste l'exposition sur la place de la danse dans la programmation du théâtre organisée en 2018 par la BnF à partir des collections détenues par le département des Arts du spectacle ou encore le formidable travail iconographique réalisé à l'occasion de la publication de l'ouvrage Chaillot, palais de la danse paru la même année.

La publication des actes des journées d'études Chaillot, lieu de tous les arts vient ainsi compléter ce travail de valorisation des archives pour en faire un ferment actif qui, tout en rejoignant le tissu d'une mémoire collective, n'en garde pas moins la saveur d'une expérience personnelle unique pour celles et ceux qui, un jour dans leur vie, ont franchi les portes de ce «palais offert au peuple». 


\section{AUTEUR}

RÉDA SOUFI

Administrateur général, Chaillot-Théâtre national de la Danse 


\title{
Le théâtre de Chaillot, les premières traces aux Archives nationales
}

\author{
Geneviève Profit
}

1 L'histoire du théâtre de Chaillot est liée à celle de la colline dont il porte aujourd'hui le nom. Il a fait l'objet de nombreuses études ${ }^{1}$, déjà anciennes ou très récentes, tant sur le lieu, sur ses activités artistiques, que sur la politique de ses directeurs.

2 Les Archives nationales conservent sur le sujet des sources écrites, iconographiques, audiovisuelles encore peu connues ou peu exploitées. La question des archives du théâtre est particulièrement délicate pour une activité éphémère par définition, le théâtre, dont l'objet même est de s'éteindre avec les lumières de la scène et de ne rester que souvenir au point que certains metteurs en scène ou directeurs de théâtre ont refusé de laisser photographier ou enregistrer leurs spectacles et n'ont laissé que très peu de traces ${ }^{2}$. Cet article s'intéressera cependant aux archives qui existent et qui ont été collectées depuis longtemps aux Archives nationales, sur support papier, permettant de mieux connaître l'histoire du théâtre de Chaillot. Elles peuvent être toujours interrogées, ou réinterrogées. Elles viennent en amont des sources versées récemment ou les complètent ${ }^{3}$. La problématique de l'image et du son est elle aussi fondamentale pour cette activité de spectacle où la parole et la musique prennent toute leur place.

3 Le théâtre de Chaillot voit sa préhistoire dans l'exposition universelle de 1878 qui s'étendait du Champ-de-Mars à la colline de Chaillot. Au milieu des autres bâtiments qui vont structurer cet emplacement, les théâtres sont un fil rouge que les archives permettent de suivre de la fin du XIX ${ }^{e}$ siècle à nos jours. Les archives en rapport avec les théâtres de Chaillot ont été versées aux Archives nationales par les administrations qui ont présidé au devenir de ces espaces, de ces spectacles et de la politique culturelle. Elles permettent d'étudier de nombreux aspects du théâtre : bâtiments, intentions et règlements, décors durables ou éphémères, techniques, effets, acteurs, textes, spectateurs... Si ces fonds présentent parfois le caractère a priori abstrait des archives de la comptabilité ou du personnel, ils comportent aussi de très nombreuses informations évoquant la réalité au plus près du terrain et de l'opinion. Cinq ensembles permettent d'écrire l'histoire du théâtre de Chaillot. Les deux premiers apportent des 
renseignements sur l'encadrement politique et sur les décisions prises au niveau des ministères. Il s'agit des fonds de l'administration des Beaux-Arts et Palais nationaux ${ }^{4}$ au sein du ministère de l'Instruction publique puis des fonds du ministère de la Culture. Trois autres fonds permettent d'étudier le/les théâtre(s) de Chaillot d'un point de vue pratique, technique et artistique: les fonds du commissariat des expositions universelles de 1878 et 1937 versés aux Archives nationales respectivement en 1890 et 1939 comportent, pour de nombreux aspects, mais principalement en ce qui concerne la construction des bâtiments, des informations très riches et encore peu exploitées par les historiens ${ }^{5}$. Le fonds du Théâtre national populaire (TNP) de 1951 à 1963, entré aux Archives nationales en 1966, présente le théâtre sous la direction de Jean Vilar6. Enfin, une première partie des fonds qui prennent la suite, sous les directions de Georges Wilson (1963-1973), Jack Lang (1973-1974), André Louis Perinetti (1974-1981) et Antoine Vitez (1981-1987) a été versée en $1990^{7}$.

\section{Les archives de la première salle de Chaillot construite pour l'exposition universelle de 1878}

4 La première salle de spectacle sur la colline de Chaillot, construite pour l'exposition universelle de 1878, est l'œuvre de Gabriel Davioud et de l'ingénieur Jules Bourdais. Elle est constituée d'une rotonde qui abrite une grande salle des fêtes de 5000 places et d'une petite salle. Les archives de l'exposition de 1878 conservent trace de cette salle : citons les albums photographiques des vues de l'exposition constitués par le commissariat et qui rassemblent les œuvres, au format de cartes postales, de différents photographes comme Neurdein, Queval, Block, Mauvillain, Nadar. Elles présentent l'extérieur et l'intérieur du bâtiment ${ }^{8}$. 
Figure 1

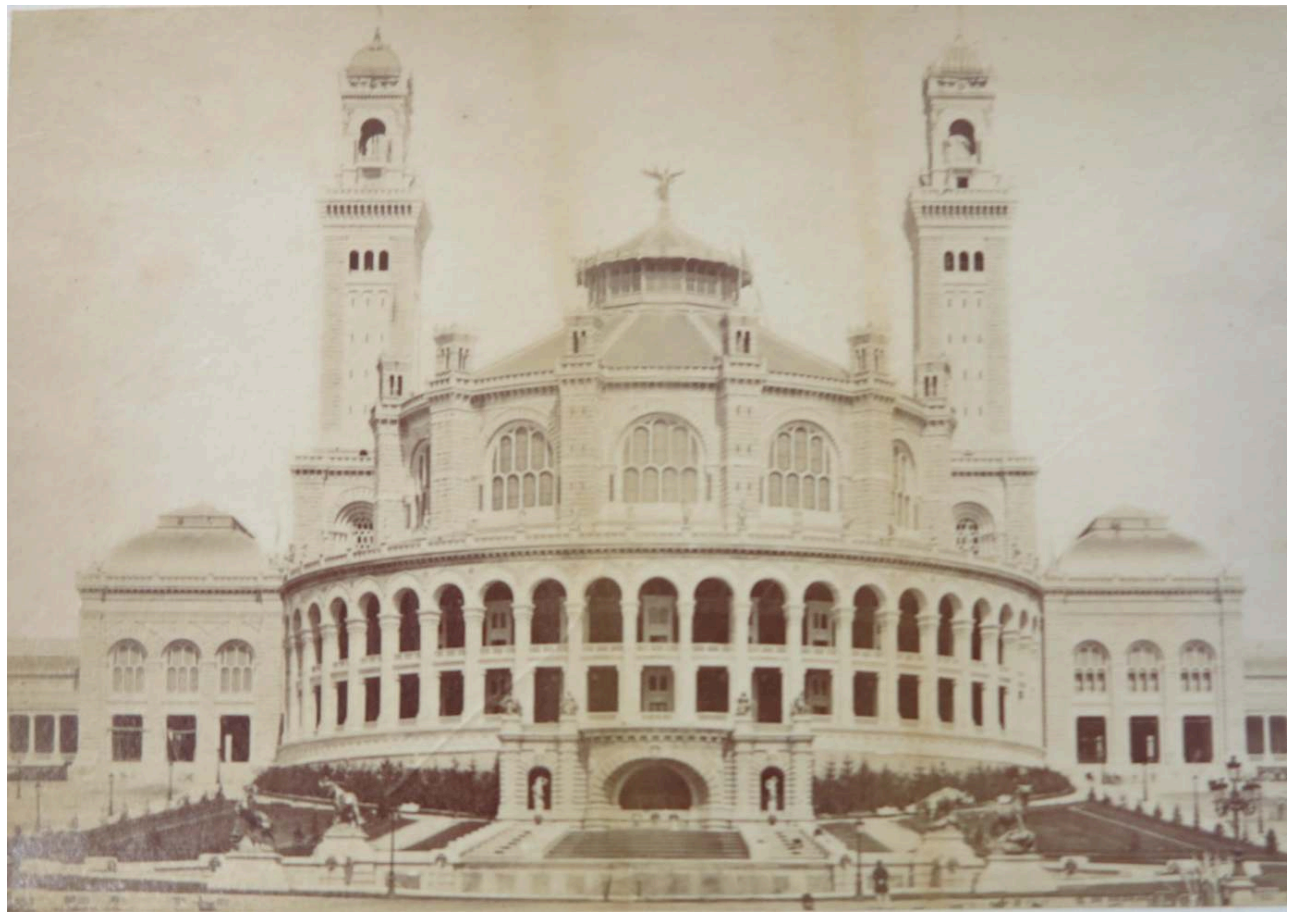

Vue extérieure de la salle de spectacle du Trocadéro, construite pour l'exposition universelle de 1878 Photographie de Nadar, 1878. Arch. nat., F/12/11909.

Figure 2

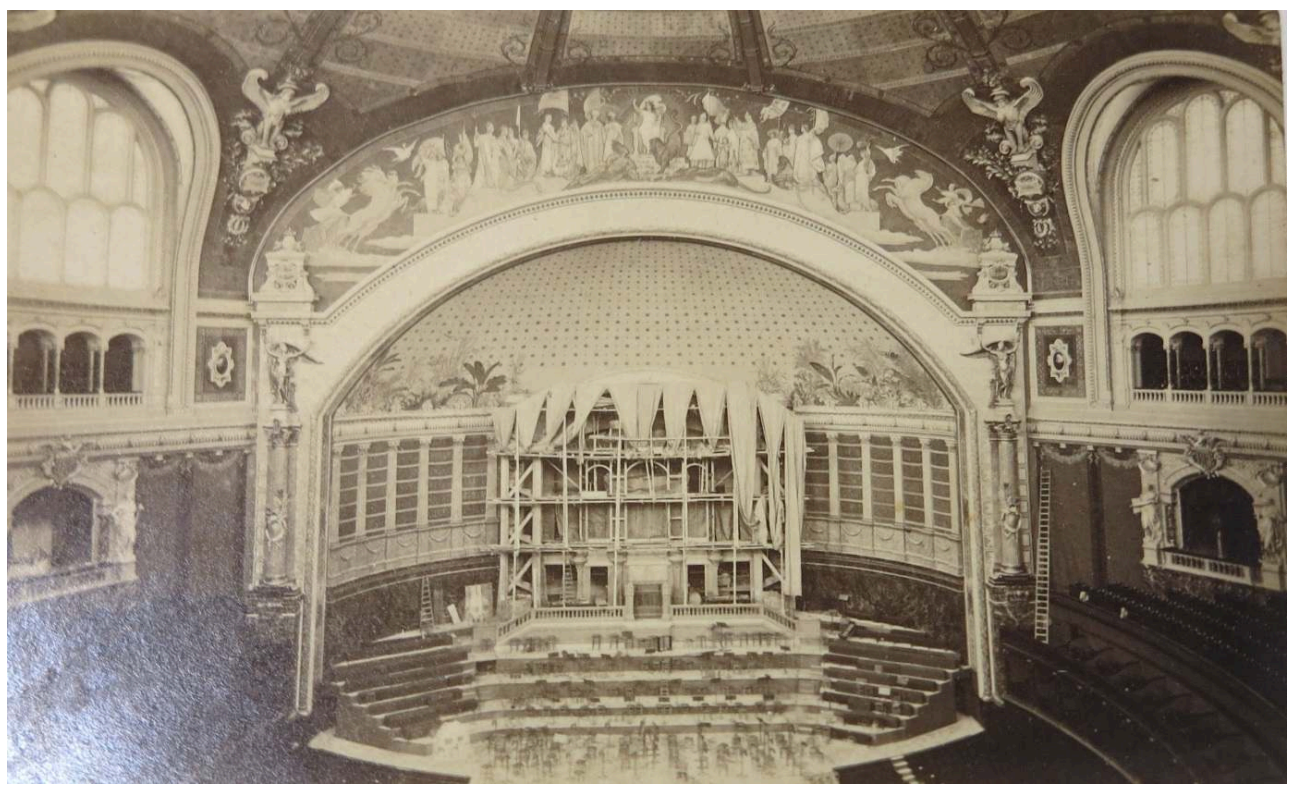

Vue intérieure de la salle de spectacle du Trocadéro, construite pour l'exposition universelle de 1878. Photographie de Neurdein, 1878. Arch. nat., F/12/11909.

Des plans de la salle ovale sont conservés, issus de l'administration des Beaux-Arts9. 


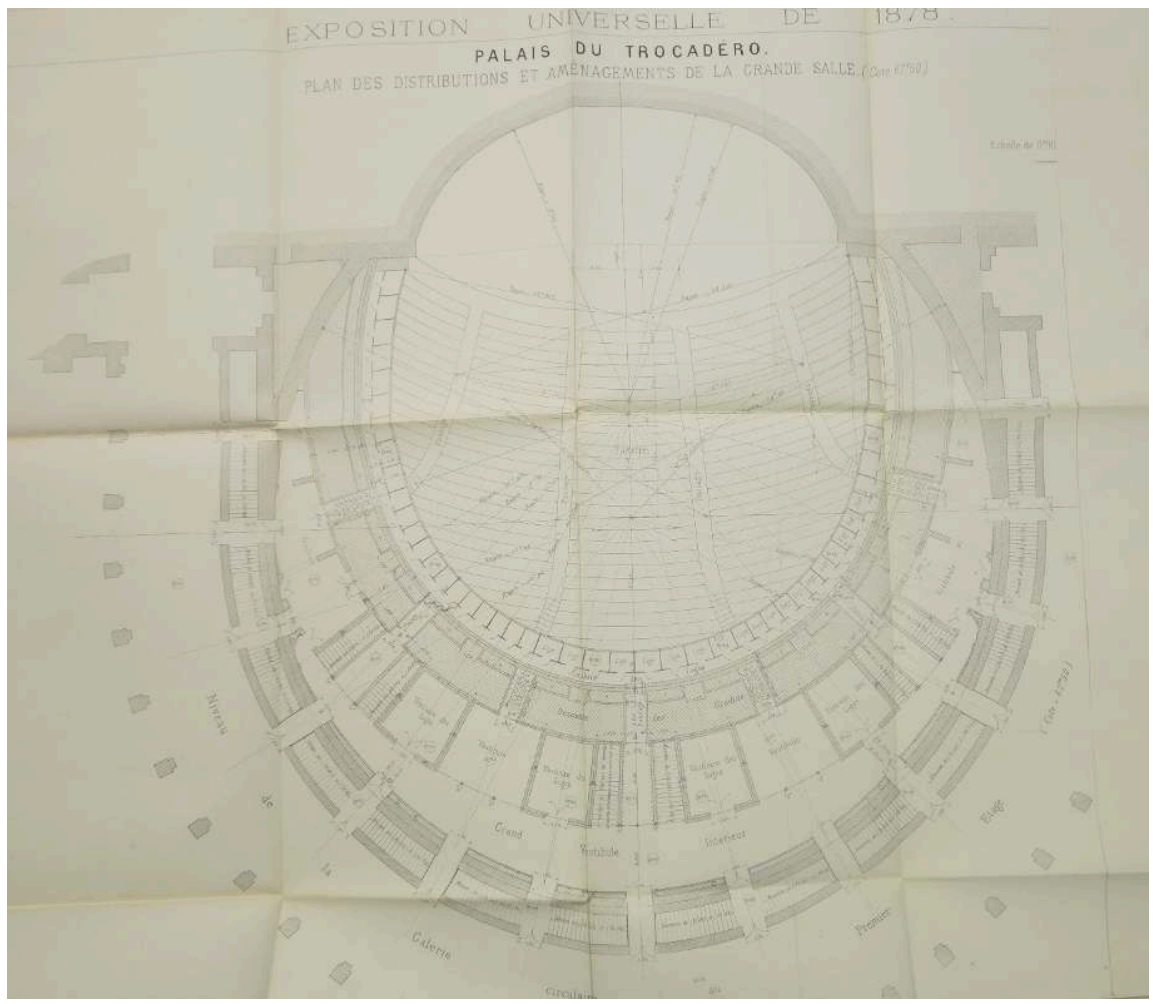

Plan de la salle de spectacles du Trocadero : plan des distributions et aménagements de la grande salle de spectacle construite pour l'exposition universelle de 1878. Arch. nat., F/21/6143.

Il y a également de nombreux dossiers relatifs à l'entretien ${ }^{10}$. On notera notamment la présence d'un courrier de la société Pleyel, Lyon et Cie du 16 février 1911 qui indique que «les derniers travaux proposés pour l'amélioration de l'acoustique de la salle des fêtes du Trocadéro viennent d'être terminés [...]. Pour parfaire cette œuvre, il restera encore à effectuer la correction relative aux ondes sonores réfléchies par la coupole et l'appropriation d'une scène isophonique ». Cette question acoustique dont l'importance est considérable a alors fait l'objet d'études successives. Une commission spéciale a d'ailleurs été mise en place dès 1903: un dossier en présente la composition et le fonctionnement à travers les procès-verbaux de ses séances de 1903 à $1912^{11}$.

7 À partir d'avril 1920 les salles de spectacle abritent un premier théâtre national populaire. Firmin Gémier en est alors nommé directeur et il est concessionnaire des salles. Il inaugure le premier théâtre national populaire dont il reste directeur jusqu'à sa mort en 1933. Ce premier théâtre national populaire ne produit pas lui-même les spectacles, il accueille d'autres théâtres (Opéra, Opéra-Comique, Odéon, ComédieFrançaise) et présente à des tarifs populaires les ouvrages du répertoire. Dans le cahier des charges ${ }^{12}$ on peut lire "Article premier: le directeur du Théâtre populaire a la concession permanente de la grande et de la petite salle des fêtes du Trocadéro et de leurs dépendances. En ce qui concerne plus particulièrement la grande salle, lorsqu'elle ne sera pas retenue pour les besoins du Théâtre Populaire, le directeur devra, avec l'autorisation du ministre, la mettre à la disposition des associations reconnues d'utilité publique. [...] Article $6:$ Le Théâtre national populaire représentera des œuvres lyriques et dramatiques appartenant au répertoire classique et moderne (œuvres françaises et étrangères). Des ballets, des concerts, des séances cinématographiques dont le 
programme devra être composé de films éducatifs, pourront également y être donnés. Article 7 : Le directeur sera tenu de donner au tarif populaire un minimum annuel de cent représentions, dont la moitié au moins au Trocadéro. Les spectacles cinématographiques et les concerts n'entrent pas en compte pour le calcul de ces cent représentations.»

8 Les archives provenant des Beaux-Arts gardent trace de la nomination de Firmin Gémier le 10 novembre 1920 et de son action $^{13}$, ainsi que des programmes de représentations jusqu'en $1930^{14}$.

\section{La deuxième salle de Chaillot : les archives de l'administration des Beaux-Arts et de l'exposition universelle de 1937, un cadre pour des spectacles et des pièces de théâtre}

9 En 1933 le théâtre ferme et les bâtiments (à l'exception des fondations) sont démolis. L'emplacement est retenu pour une partie de l'exposition universelle de 1937. Les nombreuses photographies conservées dans les archives du commissariat de l'exposition témoignent des démolitions puis de la reconstruction des bâtiments nouveaux ${ }^{15}$. Ces photographies ont été commandées par le commissariat pour mémoriser l'histoire du lieu et des travaux accomplis et illustrer le futur rapport sur l'exposition. Elles étaient réparties dans différents dossiers administratifs de l'exposition, mais en ont été extraites, une fois versées aux Archives nationales, pour des raisons de conservation. Elles ont été récemment numérisées et sont aujourd'hui disponibles pour le lecteur sur le site internet des Archives nationales. ${ }^{16}$ 
Figure 4

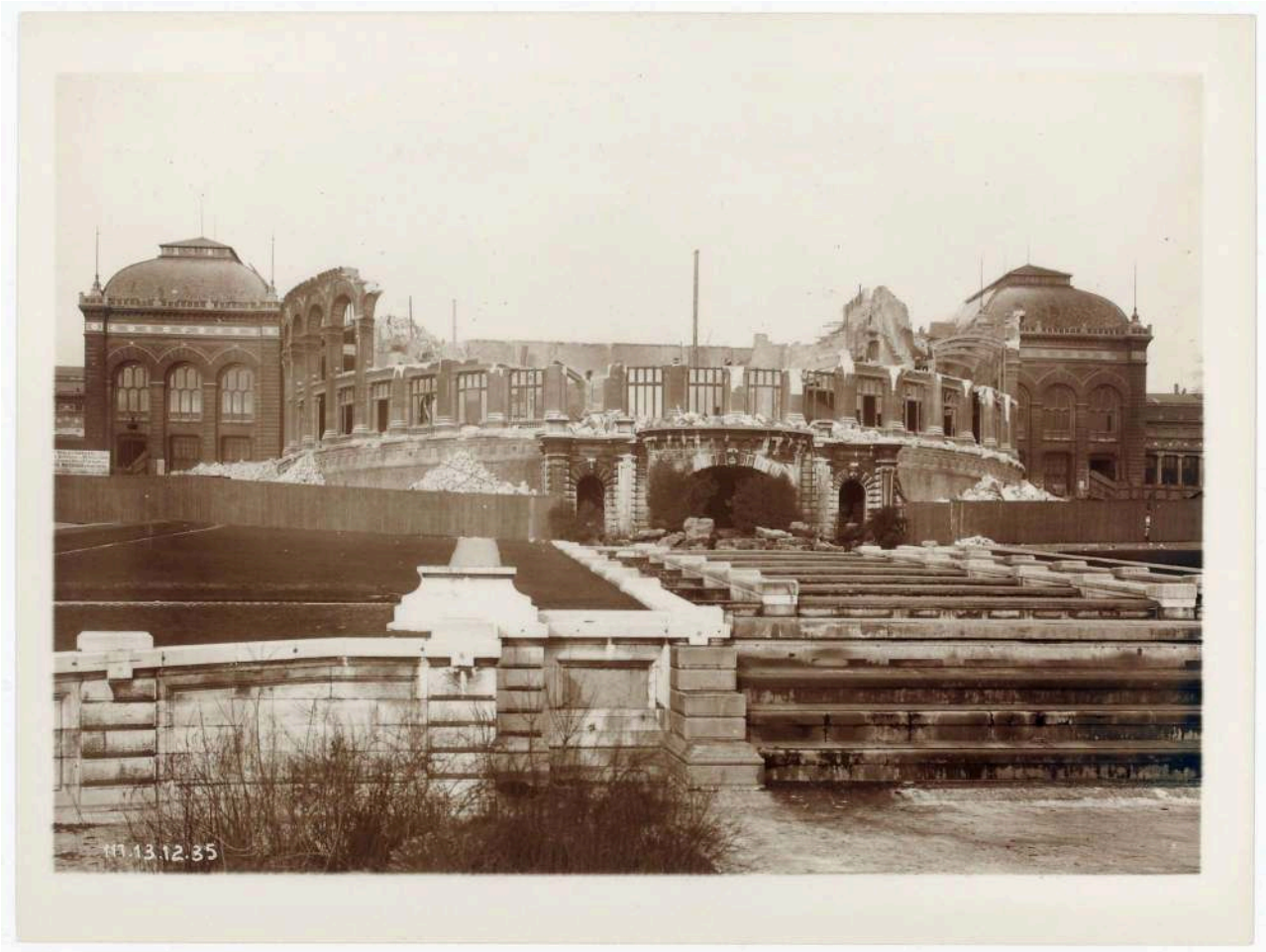

Démolitions des bâtiments de l'exposition de 1878, photographie commandée par le commissariat de l'exposition de 1937. Tirage photographique. Baranger. Arch. nat., F/12/12114.

\section{Figure 5}

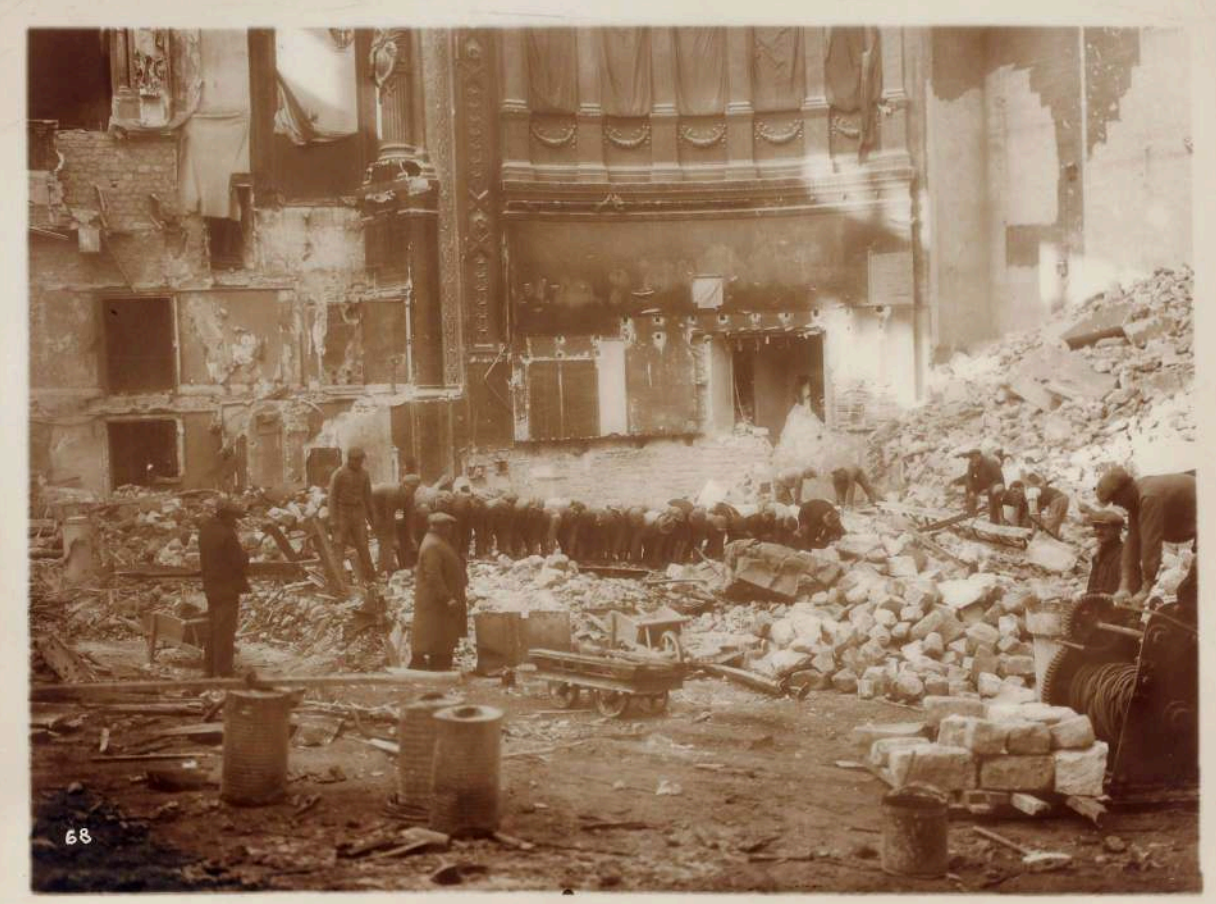

Construction des bâtiments pour l'exposition universelle de 1937, photographie commandée par le commissariat de l'exposition. Tirage photographique. Baranger. Arch. nat., F/12/12114. 
Les archives du commissariat de l'exposition de 1937 comprennent tous les projets proposés pour la construction du nouveau théâtre avec plans et élévations, ainsi que des notes qui commentent les propositions. Si certains projets sont novateurs, intégrant une salle rectangulaire, d'autres sont plus classiques, gardant la forme du théâtres à l'italienne ${ }^{17}$. Les architectes choisis finalement pour le nouveau théâtre de Chaillot sont Léon Azéma, Jacques Carlu et Louis-Hippolyte Boileau, qui ont proposé un projet moderne comprenant une très grande salle où tous les spectateurs sont face à la scène. Pour l'aménagement et la décoration de cette salle, le projet des frères Niermans est retenu. Le théâtre est presque enterré, et laisse dégagée la vue sur la grande terrasse au centre quand on regarde l'ensemble des bâtiments de la colline depuis la Seine ${ }^{18}$. Il est destiné à proposer au public des spectacles polyvalents. Moderne, il ne comprend ni loge, ni décrochement, mais inclut un balcon. Un petit livret résume le projet. En ce qui concerne l'aménagement de la salle de spectacle précédente : « on se demande encore comment on a pu donner des représentations théâtrales dans la défunte salle du Trocadéro qui, outre son acoustique défectueuse et ses dégagements difficiles, sinon dangereux en cas de sinistre, se signalait encore par son absence d'accès spéciaux (entrée des artistes, coulisses, etc.), son manque total de cintres, son impossibilité d'éclairage scénique, la forme de niche de l'espace qu'on réservait à la scène proprement dite, l'insuffisance des loges et des foyers d'artistes... ».

11 La nouvelle salle, au contraire, "sera une salle de théâtre de première catégorie, avec tous les éléments secondaires qu'elle doit comporter : scène praticable, magasins de décor, machinerie moderne, loges d'artistes confortables et hygiéniques, larges dégagements, conditionnement de l'air et forme orthophonique, etc. La fosse d'orchestre a été prévue pour cent cinquante musiciens. Les orgues seront entièrement révisés avant leur installation; ils seront électrifiés et placés d'une façon satisfaisante, tant au point de vue de leur audition que pour l'utilisation scénique de la salle. Les accès du public sont prévus de deux façons : par la place du Trocadéro [...], par les jardins [...]. Une galerie pour piétons utilisant, en partie, un passage dans les carrières situées sous le Trocadéro, a même été envisagée afin de relier la salle à la station de métro. Au-dessus du vestibule d'entrée, côté jardin, une vaste galerie-foyer, située au niveau du balcon du théâtre, pourra être utilisée comme salle des fêtes ou banquets. Des foyers secondaires, un vaste bar et une salle de réunion pour trois cents personnes complètent les annexes de la grande salle.»

Des photographies ${ }^{19}$ qui accompagnaient les archives techniques et les plans présentent des maquettes de la salle et des installations techniques, laissant voir les trappes, les cintres. Elles peuvent être comparées aux photographies conservées par l'administration des Beaux- $\operatorname{Arts}^{20}$ qui montrent le théâtre dans la réalité de sa construction en 1937. 
Figure 6

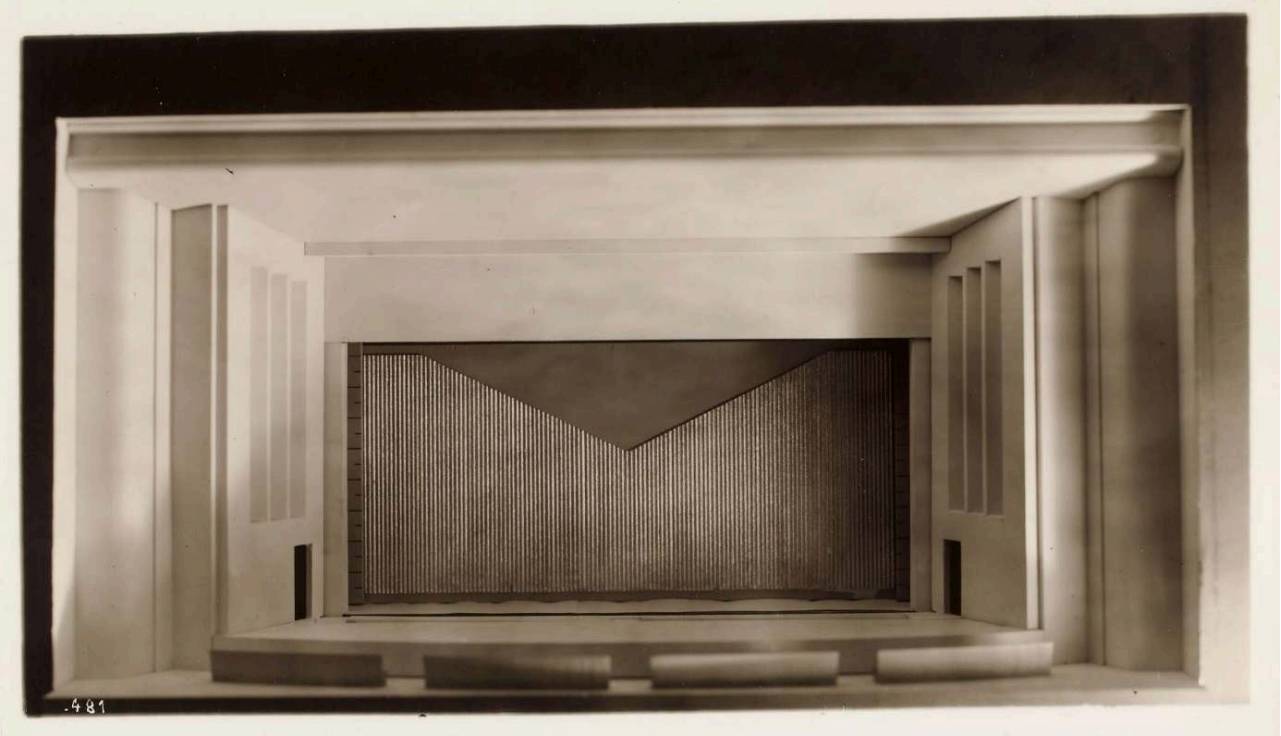

Photographie de la maquette du théâtre : vue générale. Exposition universelle de 1937. Tirage photographique, Baranger. Arch. nat., F/12/12114.

Figure 7

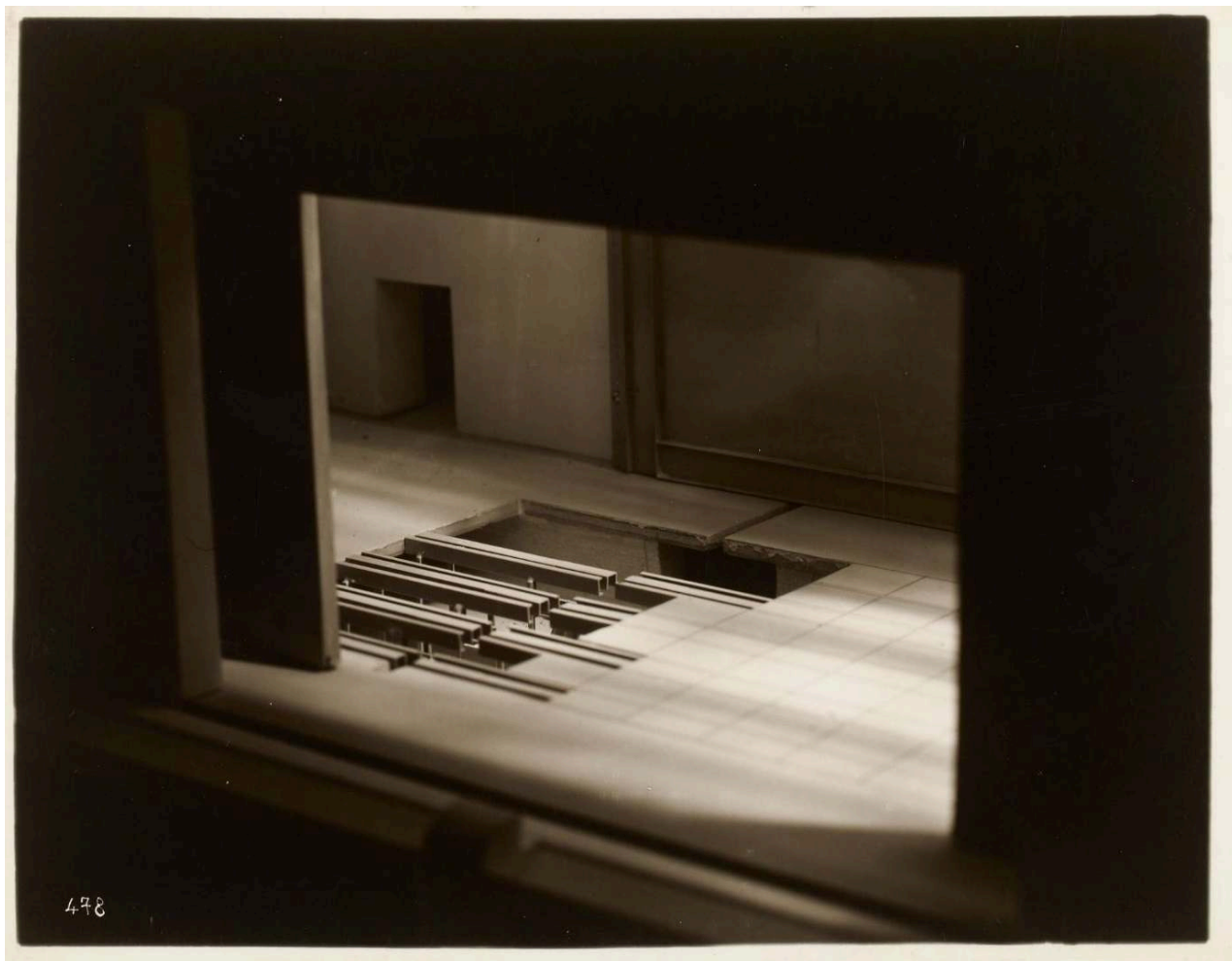

Photographie de la maquette du théâtre : vue de la machinerie. Exposition universelle de 1937. Tirage photographique, Baranger. Arch. nat., F/12/12114. 
Figure 8

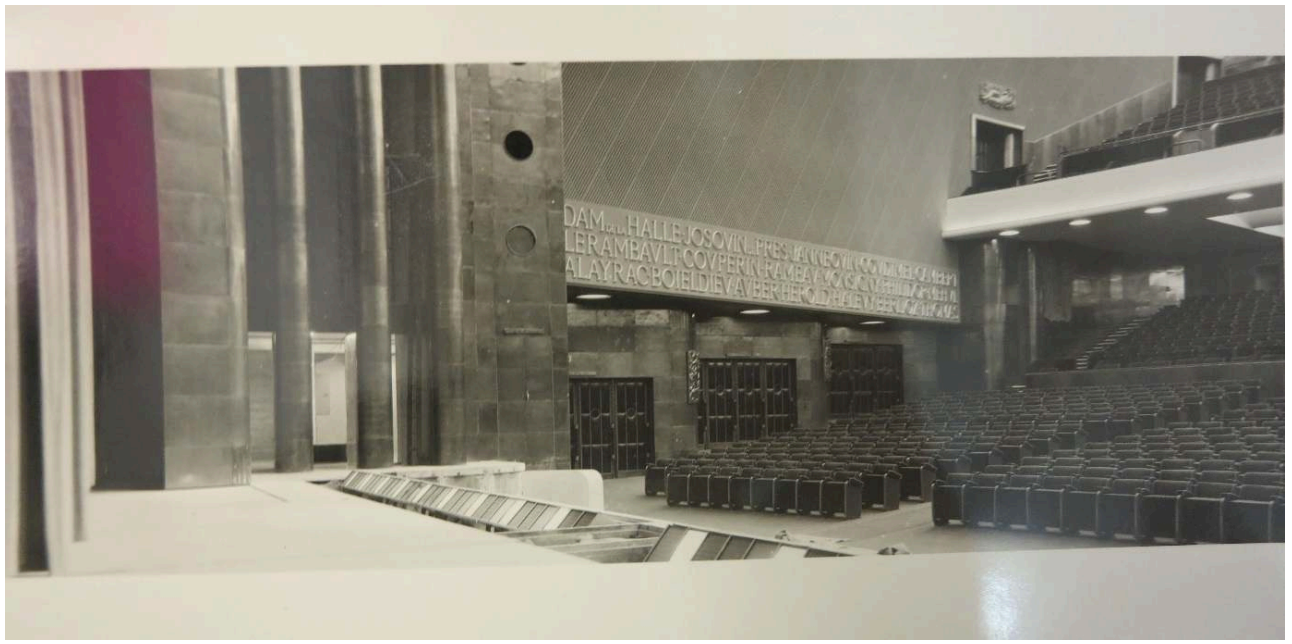

Photographies de l'intérieur du théâtre, 1938, tirage photographique. Arch. nat., F/21/6148.

Figure 9

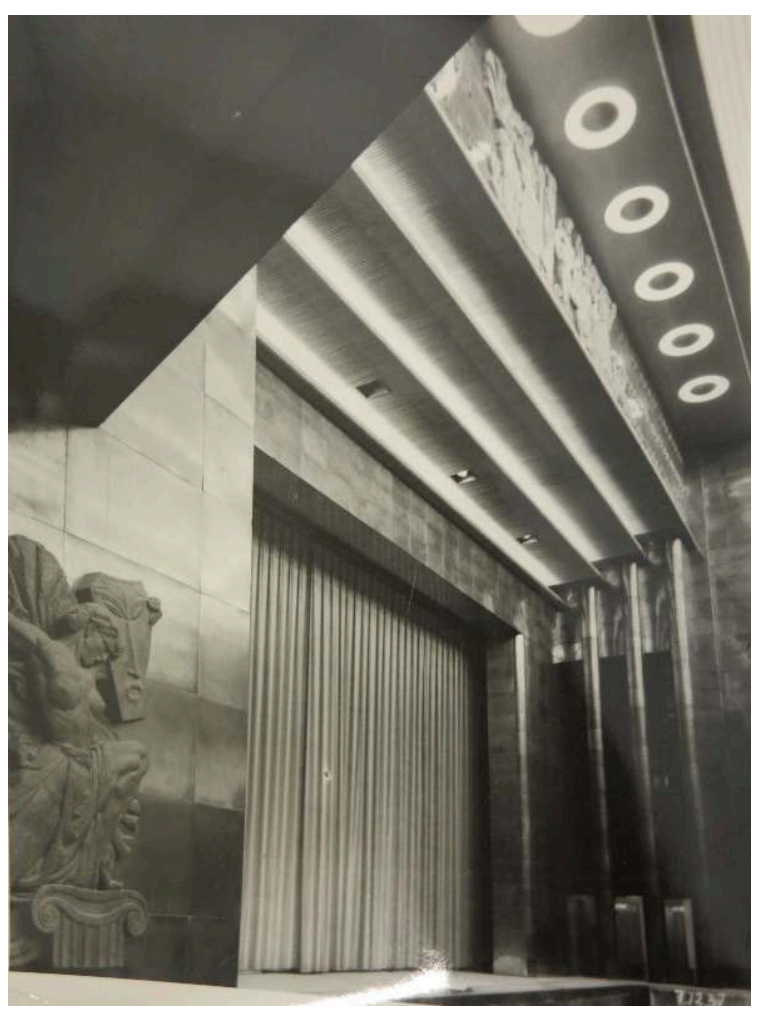

Photographies de l'intérieur du théâtre, 1938, tirage photographique. Arch. nat., F/21/6149. 
Figure 10

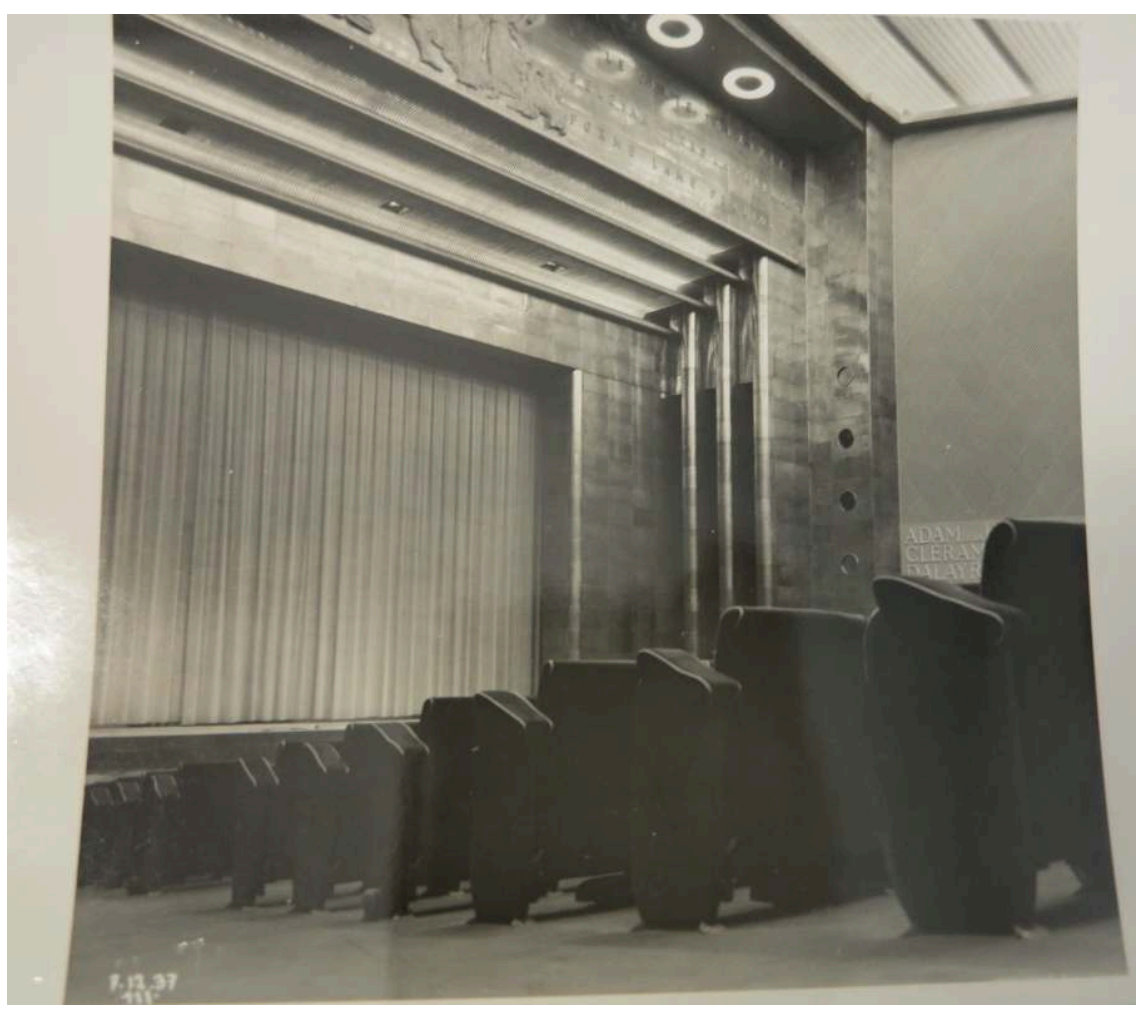

Photographies de l'intérieur du théâtre, 1938, tirage photographique. Arch. nat., F/21/6148.

13 Les archives provenant du commissariat de l'exposition de 1937 conservent donc toutes les traces des travaux du théatre de Chaillot. Elles permettent de connaître de façon détaillée les marchés, les propositions reçues, les choix techniques retenus aussi bien pour le matériel d'éclairage, que pour la maçonnerie, les aménagements et équipements scéniques, ou les choix artistiques : les dossiers comportent par exemple les dessins fait par MM. Muller et Muguet pour la décoration de la salle du théâtre ${ }^{21}$ : il s'agit de bas-reliefs représentant le rythme (un personnage à demi couché jouant de l'harmonica sur un fond de fête populaire représenté par un bal, un manège, une oriflamme, un lampion et des feuilles de chêne) et l'harmonie (une femme à demi couchée et à demi drapée ; à l'arrière-plan, la forêt, la mer et un petit village ; sur le côté droit, un petit personnage jouant du violon), insistant une nouvelle fois sur cette importance de la sonorisation. 


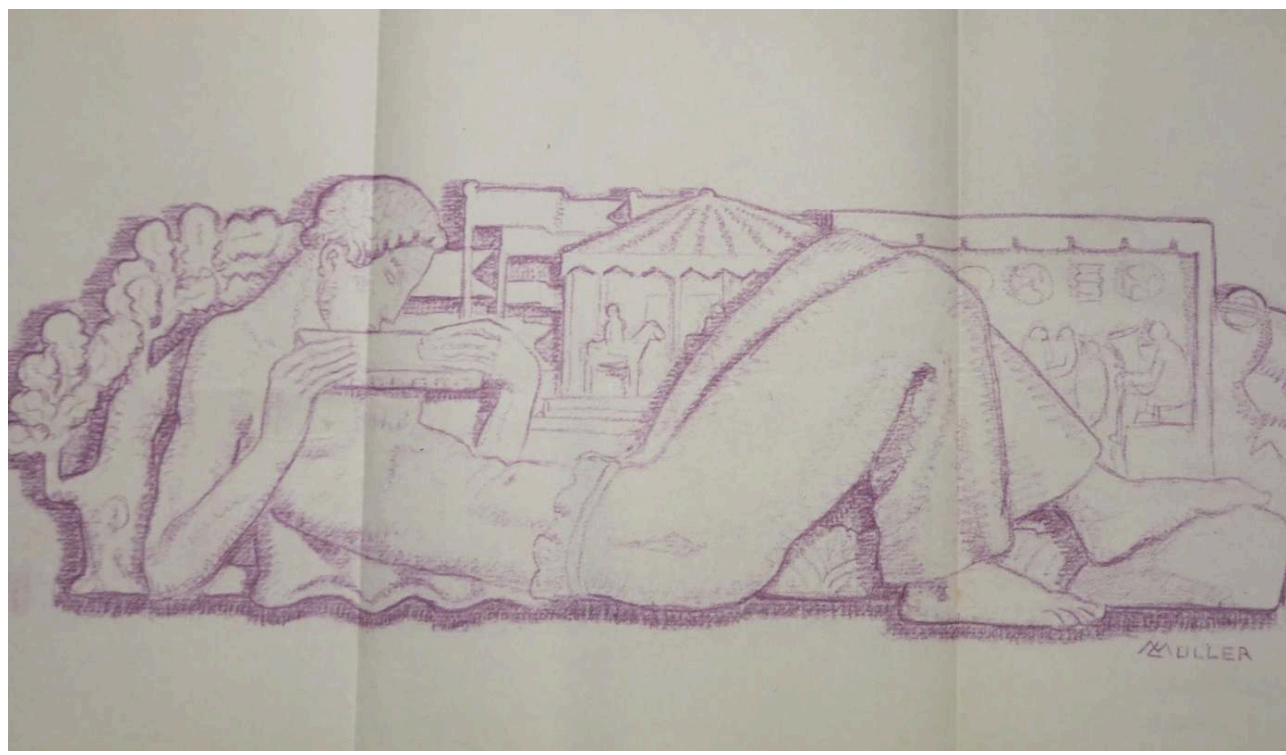

Tirage représentant les bas-reliefs proposés par MM Muler et Muguet pour la décoration du théâtre : le rythme, 1937. Arch. nat., F/12/12184.

Figure 12

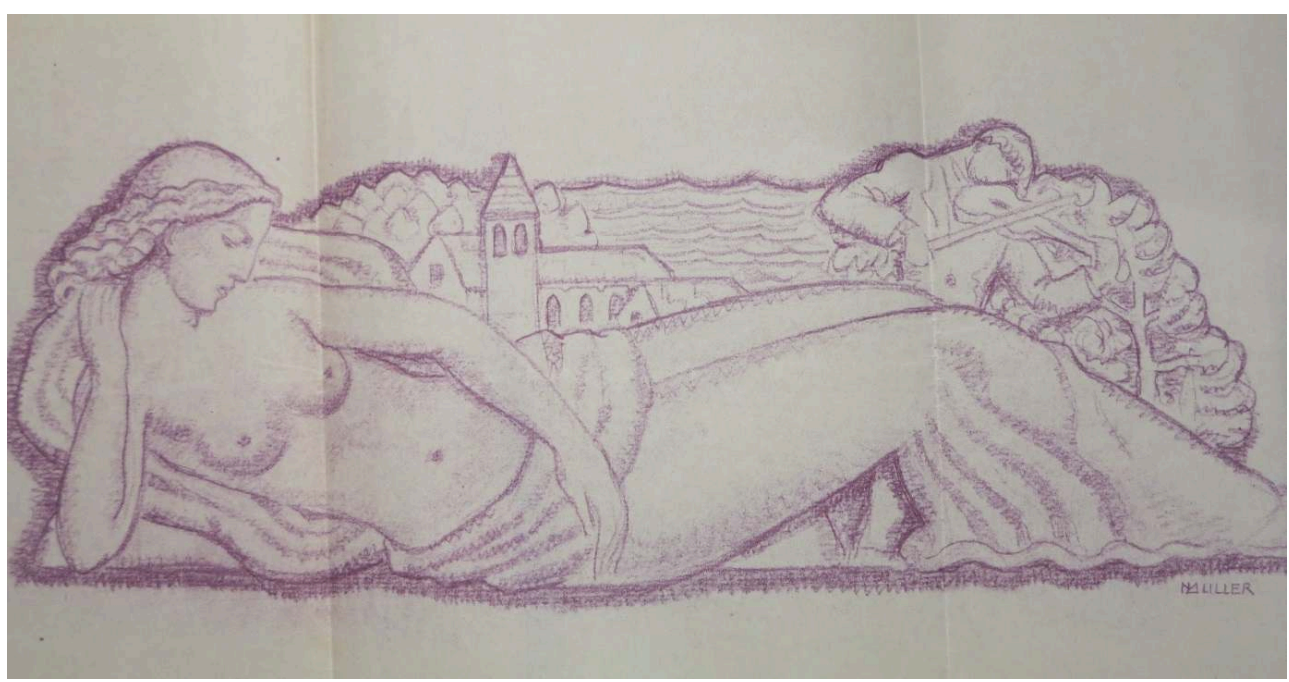

Tirage représentant les bas-reliefs proposés par MM Muler et Muguet pour la décoration du théâtre : I'harmonie, 1937. Arch. nat., F/12/12184.

Une note des frères Niermans adressée à la direction des Beaux-Arts ${ }^{22}$ revient d'ailleurs sur les difficultés posées par l'amélioration de la qualité du son: le théâtre mesure 41 mètres de longueur et 37 mètres de largeur pour un volume de $19000 \mathrm{~m}^{3}$. "Cette salle devait servir à de multiples usages et devait permettre, en particulier, d'avoir une salle d'audition d'orgues et de grand orchestre, parfaite en tous points, et, d'autre part, une salle de théâtre sur plateau de laquelle on pouvait jouer tous les répertoires. [...] Au point de vue acoustique le problème posé a amené les architectes et leur ingénieur conseil acousticien, Jacques Brillouin, à trouver une solution inédite qui s'adapte à un problème nouveau, la sonorité de la salle qui doit être parfaite, ne devant pas être la même pour une audition de grand orchestre, d'orgue ou de comédie ». La solution 
technique choisie est décrite de façon détaillée: elle évite tout écho, permet une audition à toutes les places, une réverbération convenable et, pour certains usages particuliers, rend la salle plus sonore.

On notera que de nombreuses coupures de presse rassemblées de façon thématique témoignent des oppositions qui se sont élevées contre la construction de la nouvelle salle. Une pétition y répond, intitulée « protestation contre les tentatives d'obstruction apportées aux travaux de reconstruction du nouveau Trocadéro actuellement en cours » et dont le premier signataire est Paul Valéry. Elle associe les travaux du théâtre au projet global de rénovation du site du Trocadéro et en particulier aux musées : « les signataires s'associent aux conservateurs des musées pour protester vivement contre les tentatives de dénigrement de l'œuvre entreprise qui risquent de compromettre gravement l'effort poursuivi par les pouvoir publics en vue d'assurer le succès de la grande manifestation internationale prévue pour $1937 »^{23}$. Le directeur de ce nouveau théâtre est Paul Abram.

L'ensemble de ces archives, issues de l'administration, renseigne le lecteur principalement sur le contexte du théâtre, politique, architectural, administratif, financier, technique. Il permet d'évoquer la salle, les lieux et les conditions du spectacle. Mais la pièce elle-même, le jeu, n'apparaît pas. Les archives conservées aux Archives nationales pour cette première période ne permettent pas de connaître le théâtre de Chaillot dans la forme éphémère du spectacle, celle qui fascine le public quand il regarde, avant que l'historien ne souhaite l'étudier avec le recul du temps.

\section{Les archives de Jean Vilar : des archives du spectacle}

17 Après cette première période qui se termine avant la Seconde Guerre mondiale, les archives conservées aux Archives nationales relatives au théâtre de Chaillot nous conduisent en 1951. À cette date, Jeanne Laurent, alors sous-directrice des spectacles, nomme Jean Vilar directeur du Théâtre national populaire, le TNP. En 1966 les archives du théâtre seront confiées aux Archives nationales par Jean Vilar lui-même. La volonté de ce dernier de voir conservée la mémoire du TNP, expérience nouvelle et révolutionnaire, transparaît dans les archives mêmes. La variété et le caractère exhaustif des séries de documents conservés en témoignent, qu'il s'agisse de l'administration, du théâtre, de la mise en scène ou du public ${ }^{24}$.

Les archives administratives du théâtre de Jean-Vilar présentent d'abord des informations sur les bâtiments et leur entretien ${ }^{25}$, sur la comptabilité et les budgets. La série des comptes et bilans semble complète. Les dossiers de paiements du personnel sont tous conservés ${ }^{26}$. Il existe plusieurs types de dossiers de personnel suivant les statuts, les métiers, etc. Tous les métiers sont présents: accessoiristes, buralistes, contrôleurs, costumiers, décorateurs, habilleuses, régisseurs, électriciens, gardiens, machinistes, musiciens, comédiens, etc. La position de Jean Vilar face aux éventuels conflits apparaît dans la correspondance et les notes. Le 18 décembre 1954 par exemple, les 32 ouvreuses du théâtre adressent une pétition à la direction pour se plaindre de la suppression des pourboires, à la suite d'un arrêté du ministre du Travail relatif à leur rémunération et à leur sécurité sociale ${ }^{27}$. La réponse de Jean Vilar le 30 décembre 1954 maintient la décision, mais précise et module les modes de fonctionnement: " la rémunération au pourboire des ouvreuses est supprimée à partir du 2 janvier 1955. [...] Mesdames les ouvreuses s'engagent à ne pas accepter le moindre pourboire, à 
l'occasion de ces représentations, et à continuer d'exercer leur métier avec la plus parfaite conscience professionnelle. [...] Mesdames les ouvreuses seront rétribuées chaque semaine, par partage égal, entre elles, du montant résultant du cumul de la perception d'une somme de douze francs par billet. [...] Dans le cas où, par semaine et par ouvreuse, le salaire brut acquis demeurerait inférieur au salaire syndical considéré pour l'ensemble des représentations de la semaine, ce dernier serait automatiquement acquis. [...] Ayant pris bonne note de votre attitude individuelle à l'occasion de la récente pétition que vous m'avez adressée, je vous saurais gré, Mesdames, de bien vouloir signer individuellement ce document, faute de quoi il me faudrait me rabattre sur une proposition de salaire normale.». Des affichettes feront connaitre cette décision au public.

Figure 13

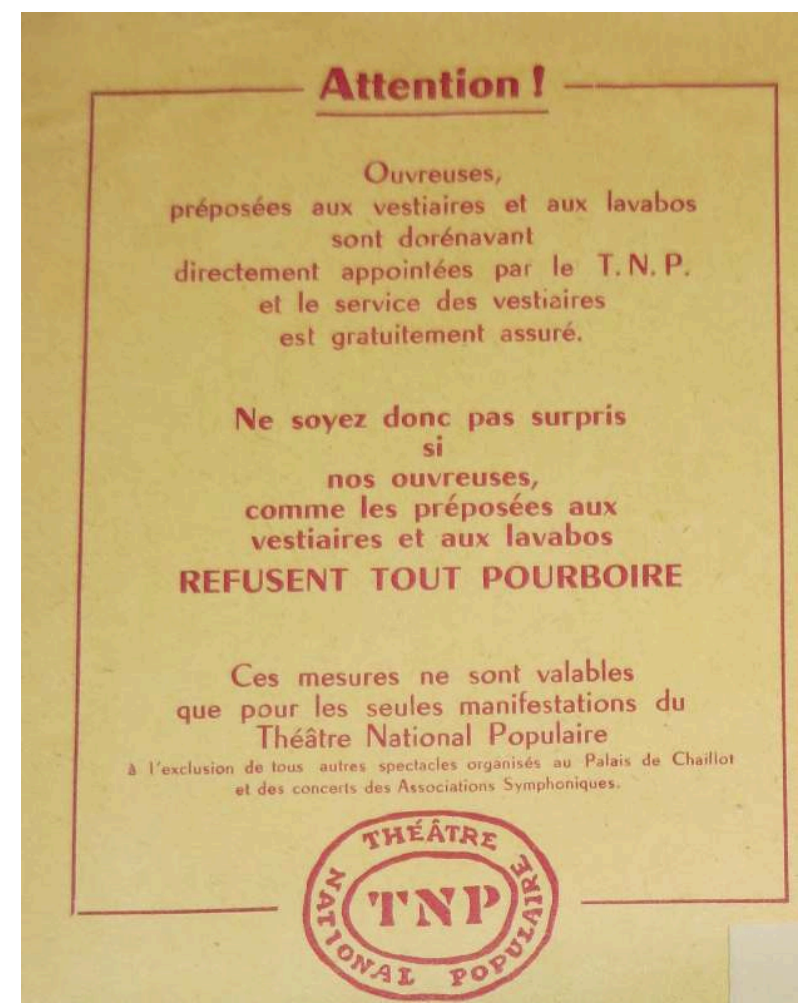

TNP, affichette indiquant que les ouvreuses n'acceptent pas de pourboire, 1955. Arch. nat., 295AJ/257.

Les relations de Jean Vilar avec ses collaborateurs sont très présentes: des dossiers aujourd'hui classés par catégories (chorégraphes, danseurs, choristes, musiciens, comédiens) et au nom des artistes ont été conservés. Le lecteur trouvera par exemple dans le dossier de Maurice Jarre, régisseur de la musique, une lettre du 20 mars 1963 signée par Jean $\operatorname{Vilar}^{28}$ adressée à "Mon cher Maurice bien-aimé ». Il y déplore son absence. Après avoir pris la décision d'arrêter le payement de ses mensualités, il donne son sentiment: "Je trouve anormale ta position. Deux concerts TNP-RPF sans ta présence aux répétitions et concerts... et le reste! Tu n'es pas membre correspondant du TNP. Et d'autre part, passer par mois une demi-heure à Chaillot ne peut expliquer, selon notre accord, un salaire de 202000 anciens francs [...]. Non seulement tu t'es absolument désintéressé des travaux quotidiens de cette régie, mais encore tu te désintéresses des grands événements qui incombent à cette régie. Et c'est nous qui, 
avec du retard bien sûr en assumons les tâches. C'est trop Maurice ». La correspondance fait apparaître ici des relations qui mélangent la bienveillance de l'ami et les obligations du directeur.

Ses relations avec des artistes extérieurs majeurs présentent également un grand intérêt. En 1961 par exemple, il demande à Alexandre Calder de concevoir une oriflamme pour le TNP et pour Avignon ${ }^{29}$. L'échange est rapide : «Je serai très content de faire quelque chose pour Vilar", écrit Calder, "est-ce que l'oriflamme doit dire quelque chose ou être aussi beau que possible? ?. La réponse indique « l'oriflamme doit être ainsi que vous le dites, aussi gaie que possible, mais une liberté totale vous est laissée pour sa composition. Il serait bien, toutefois, qu'elle évoque soit le TNP, soit Avignon, mais ce n'est pas absolument obligatoire ». Des relations de collaboration un peu distantes qui seront nuancées de l'envoi ultérieur d'une carte plus personnelle. Un autre dossier permet de connaître les relations de Vilar avec le peintre Édouard Pignon. De nombreuses autres personnalités artistiques ou littéraires sont présentes, qui ont pu collaborer à tel ou tel spectacle ${ }^{30}$.

21 Les archives de Jean Vilar font donc apparaître à la fois une administration du personnel complexe et un réseau social professionnel, artistique voire amical très développé, qui s'étend même au-delà de la France, quand la troupe du TNP collabore avec le Piccolo Theatro de Milan ${ }^{31}$. Les tournées en France et à l'étranger sont nombreuses et, pour chacune, un dossier a été constitué32. Certains dossiers présentent les activités du TNP au Festival d'Avignon, d'autres évoquent des projets qui n'ont pas toujours abouti. Les spectacles musicaux sont aussi présents car Jean Vilar s'intéressait particulièrement à la musique ${ }^{33}$. Tout est conservé.

Les aspects artistiques des spectacles constituent une grande part du fonds d'archives versé par Jean Vilar. Les dossiers sont classés par pièce de théâtre. Le souci de Jean Vilar de témoigner de ses expériences et innovations théâtrales apparaît clairement quand on considère la masse de documents conservés. Pour qui s'intéresse aux dossiers relatifs à la mise en scène, les indications sont extrêmement détaillées. Ces archives permettraient à elles seules de reconstituer et remonter le spectacle. Les accessoires, la figuration, les indications scéniques sont conservées dans des livrets où textes, indications écrites de mise en scène et de décor et indications scéniques sont associés. Des croquis montrent l'emplacement des acteurs, la mise en scène est explicitée avec des schémas et des indications très précises ${ }^{34}$. Tous les détails sont notés par écrit. La scène est représentée très symboliquement et des flèches indiquent le parcours des acteurs. L'inventaire des accessoires complète l'ensemble. Il existe aussi des listes des acteurs présents pour chaque représentation. 


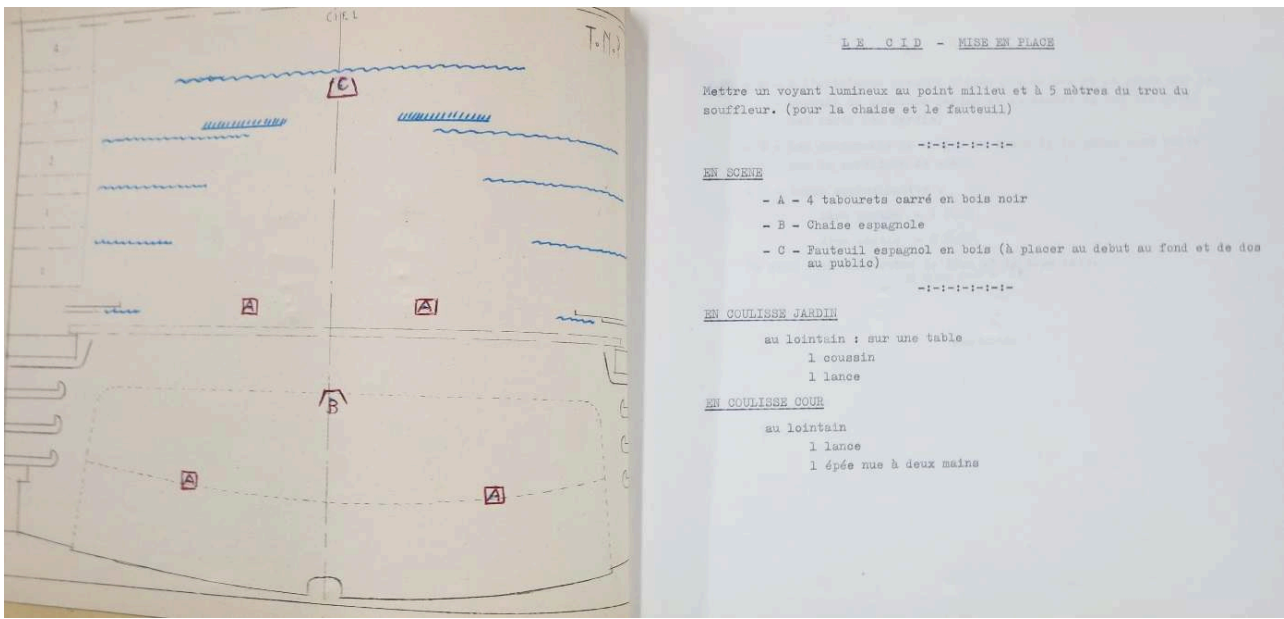

Représentation du Cid, livret comportant les indications scéniques pour la mise en place, 1958. Arch. nat., $295 \mathrm{AJ} / 474$.

\section{Figure 15}

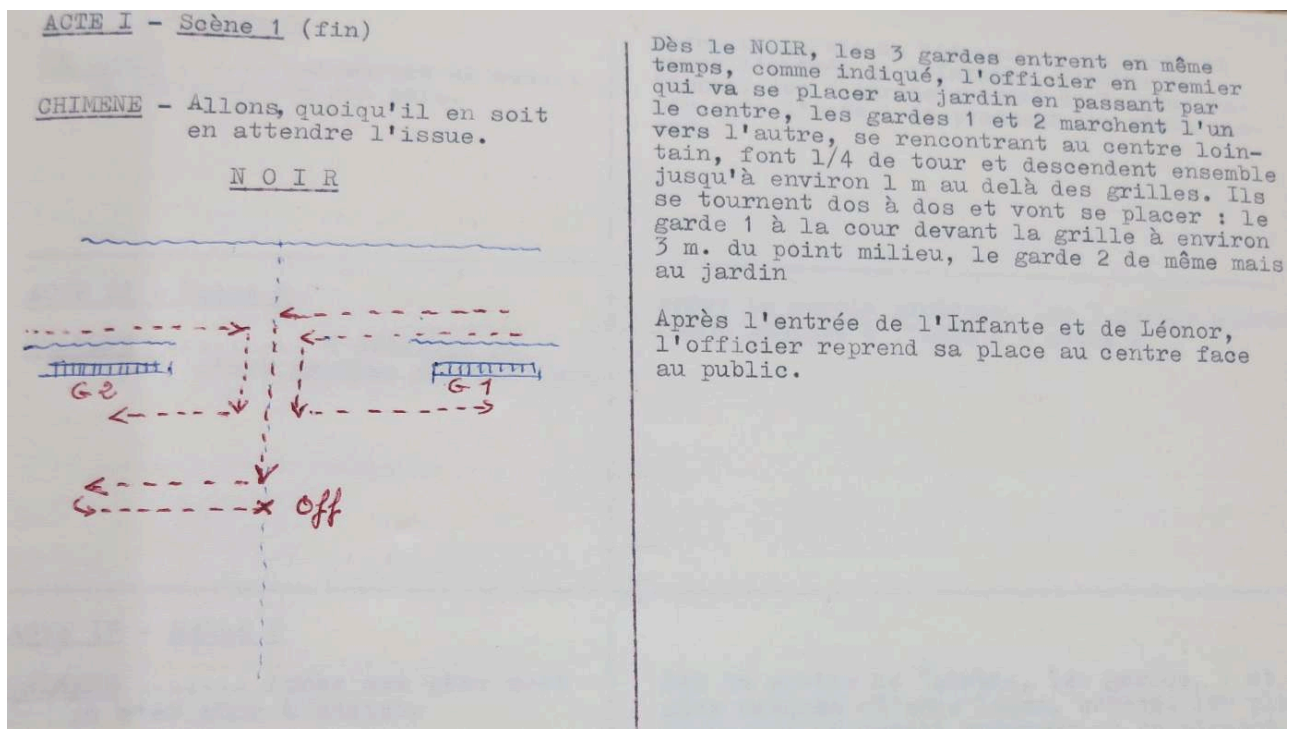

Représentation du Cid, livret comportant les indications scéniques pour le début de l'acte I scène 1 , 1958. Arch. nat., 295AJ/474.

Si ces éléments permettent de connaître la mise en scène en amont de la représentation, dans le travail préparatoire, une autre série de documents complète cet ensemble, les photographies prises principalement par Agnès Varda qui a travaillé pour le TNP à la demande de Jean Vilar, en même temps que Sabine Weiss, Jean-Pierre Lenoir, Mario Atzinger ou le studio Bernand ${ }^{35}$. Elle a photographié les spectacles de Jean Vilar pendant toute la période du TNP, constituant de véritables reportages. Ses prises de vue sont un regard au plus près du spectacle, de son ambiance, des moments forts de la pièce ou de la mise en scène. Mais Agnès Varda s'est également intéressée aux marges de la mise en scène, aux répétitions parfois (Lorenzaccio par exemple). Certaines photographies ont participé de la gloire ou de la légende d'artistes comme pour Gérard Philipe. Les vues d'acteur en costume étaient nombreuses, beaucoup servaient à la communication du TNP et étaient largement publiées. Certains albums 
comportent exclusivement les photographies d'un acteur. Si certaines des photographies existent sous forme de tirages, toutes sont rassemblées sur planchescontacts $^{36}$. Les regarder ainsi, comme une démultiplication des moments du spectacle, permet d'entrer véritablement dans la réalité du travail du photographe et des acteurs : poses, attitudes, mouvements qui semblent suspendus voient parfois leur achèvement dans la photographie suivante ou invitent à faire preuve d'imagination. Ces photographies étaient utilisées pour la communication du TNP, reproduites sur les programmes ou par les éditeurs. Elles pouvaient aussi servir pour la confection de films. Le TNP a été l'objet de demandes de tournages, d'enregistrements et d'émissions dans les media (discographie, radio, télévision, cinéma). En 1957 par exemple un reportage télévisé sur Lorenzaccio est monté par l'ORTF. Un courrier de l'administration des programmes de l'ORTF indique que la retransmission du spectacle sera limitée à 30 minutes et que les acteurs seront payés par cachet. Le dossier comprend la liste des cachets et les modalités d'installation des services techniques au théâtre.

Les « dossiers de pièces $»^{37}$ conservent trace également des rapports avec les media et de la communication autour des spectacles. Il s'agit, pour chaque pièce ou chaque spectacle, de rassembler toute la documentation qui est venue s'ajouter aux représentations: affichettes, programmes, publications du nom des acteurs, cartons d'invitation, extraits de pièces, résumés et photographies. Le dossier de L'Avare de Molière créé en 1962 comporte aussi un prospectus qui invite à venir profiter de la pièce pour les fêtes de fin d'année: « 3 spectacles, 2 apéritifs, 1 nuit dansante pour 1000 francs! Passez les réveillons au Palais de Tokyo ». Le dossier de la pièce de Bertold Brecht, La résistible ascension d'Arturo Ui, en novembre 1960, comprend des extraits de presse : pour Le Parisien, il s'agit d'" un spectacle qui fera date dans l'histoire du TNP. [..] Grande réussite de la troupe tout entière ». Pour Combat, « nous sommes sortis du Palais de Chaillot bouleversés, nous avons retrouvé le Vilar (animateur et comédien) des grandes créations. Certaines entrées d'Arturo Ui, encadré, serré par ses hommes de main, donnent le frisson. Dans sa simplification, à laquelle le travail de Vilar et Georges Wilson se conforme, rien de plus théâtral que ce théâtre créateur d'images percutantes dont les répliques des personnages sont comme la légende ».

Enfin de façon très originale les archives du TNP comportent des dossiers de sondage ${ }^{38}$ intitulés «questionnaires de satisfaction»: le public pouvait remplir, à la fin des représentations, des feuilles de sondage où chacun donnait son avis sur la pièce. Ces avis, de longueur très variable, parfois critiques, mais le plus souvent élogieux, étaient signés et indexés selon la catégorie professionnelle ou sociale des signataires. Ces fiches ont été recopiées avec soin dans des registres qui se présentent par ordre chronologique et par pièce. La volonté de connaitre le ressenti du public et l'image du théâtre apparaît très clairement. Cet ensemble rappelle à quel point Vilar est tourné vers son public et veut faire de son théatre un théatre populaire en même temps qu'il constitue un témoignage du succès de son entreprise. Des dossiers relatifs aux relations avec le monde du théâtre, le monde politique, les associations, les musées, tous les milieux, témoignent de la volonté de Jean Vilar de faire un théâtre populaire et de son implication ou de son influence dans l'ensemble de la vie culturelle de son temps ${ }^{39}$.

Le fonds des archives de Jean Vilar est tourné vers le fonctionnement interne du théâtre, vers les représentations, mais il regarde aussi beaucoup vers l'extérieur, vers tous les publics. 


\section{Le théâtre de Chaillot après Jean Vilar : les promesses des archives versées en 1990}

En 1963 Jean Vilar renonce au renouvellement de son contrat. Il est remplacé par Georges Wilson qui ouvre une salle supplémentaire, la salle Gémier (500 places) destinée à la présentation d'œuvres contemporaines. Le décret 68-906 du 21 octobre 1968 transforme le TNP en établissement public relevant directement de la tutelle du ministère de la Culture. Les missions du théâtre de Chaillot sont alors de «favoriser le renouvellement des formes et des conditions de la création artistique contemporaine ». Vont se succéder à sa tête Jack Lang (1973-1974), André Louis Perinetti (1974-1981), Antoine Vitez (1981-1988), remplacé par Jérôme Savary. En 1990 les versements d'archives qui nous occupent ici s'arrêtent. Le seul fonds versé en $1990^{40}$, qui a permis pendant longtemps de proposer aux lecteurs quelques éléments sur cette période $d u$ théâtre de Chaillot, a pour origine la direction du Théâtre et des Spectacles du ministère de la Culture. Il comporte une très importante correspondance, des dossiers de contrats de comédiens entre 1963 et 1984, des dossiers de budget et de comptabilité, quelques plans du théâtre de Chaillot et de ses modifications et aménagements entre 1972 et $1981^{41}$, ceux des dossiers de production de spectacles entre 1974 et 1984.

Figure 16

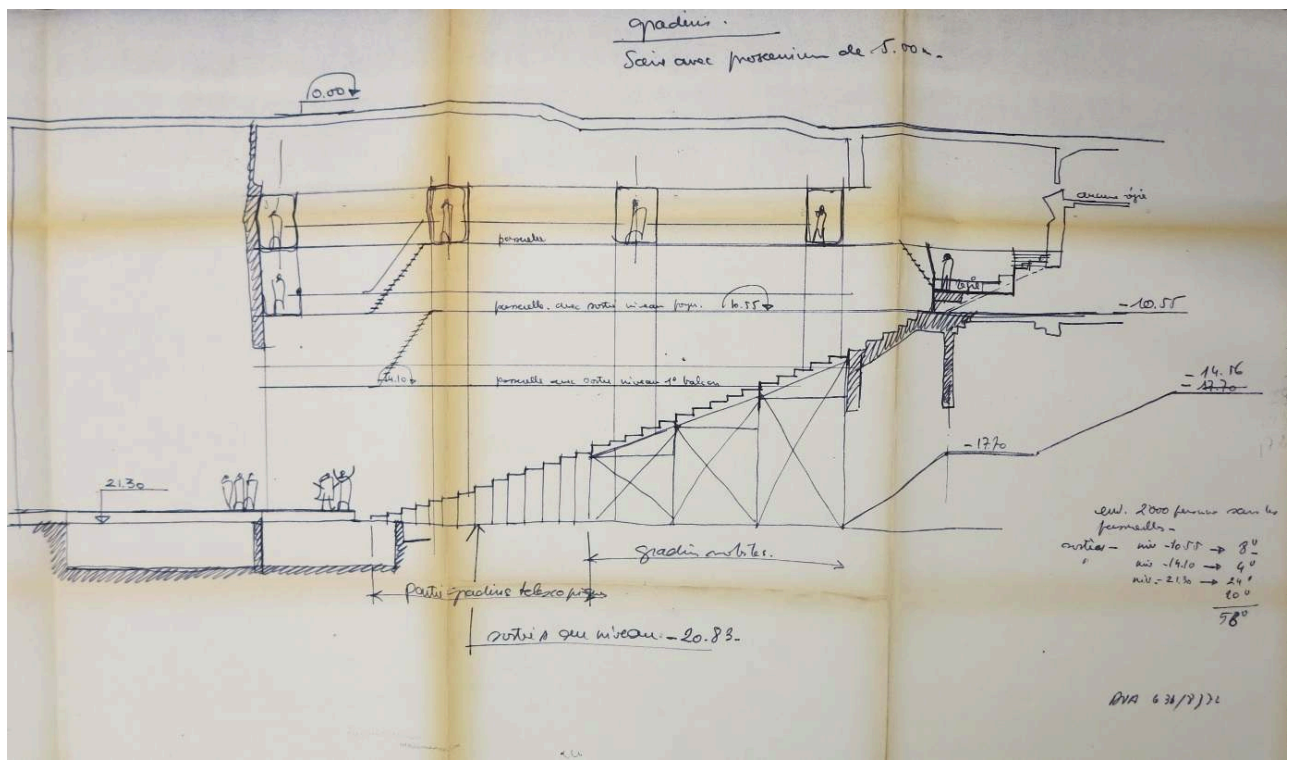

Élévation représentant les projets de modification de la salle de Chaillot, 1972. Arch. nat. 19900195/109.

Mais ces dossiers restent lacunaires. Notons cependant un dossier original relatif à l'enregistrement de vidéos et l'introduction de nouvelles pratiques au théâtre. En 1981, avait déjà été enregistré par le théâtre de Chaillot, L'École des femmes, Tartuffe et Don Juan ${ }^{42}$. En 1983, une brochure ${ }^{43}$ intitulée Office culturel pour la communication audiovisuelle (OCCAV), Vidéo sur scène, 8 mars 1983 résume un nouveau programme d'enregistrements mis en place à la demande et avec l'aide financière de la direction du Théâtre et des Spectacles du ministère de la Culture. L'Occav est alors chargé de préparer et de mettre en œuvre une large politique d'archivage audiovisuel d'œuvres 
de scène notamment en matière théâtrale. Il s'agit d'une expérience de création audiovisuelle qui permet de constituer une mémoire de la création théâtrale hors du cadre traditionnel de retransmission de la télévision. Pour les spectacles retenus, trois caméras électroniques sont disposées dans la salle et enregistrent le spectacle en continuité. Une captation est réalisée en une seule journée sans spectateurs. Aucune modification n'intervient dans le déroulement de la pièce et le jeu des comédiens. Les captations restituent le spectacle tel qu'il est présenté au public du théâtre. Afin de conserver en mémoire l'œuvre et son environnement, elle est toujours enregistrée avec la même approche: prise de vue de l'affiche, du spectacle et interview de l'auteur, du metteur en scène ou des acteurs principaux. Il est prévu que trois vidéogrammes soient tirés en U-matic et puissent être visionnés. Un vidéogramme sera remis au producteur de spectacle, un à la Bibliothèque nationale (maison Jean Vilar), un conservé par l'Occav. Une bande mère constitue le document de la captation originale. Le réalisateur, Paul Robin Benhaïoun, a déjà réalisé plus de quarante dramatiques à la télévision.

En 1982, parmi les huit spectacles enregistrés par l'Occav à Paris, figure au théâtre de Chaillot la pièce Le père d'Auguste Strindberg dans une nouvelle mise ne scène d'Otomar Krejca et dans une traduction de Jacques Robnard. Le 8 mars, au Centre Pompidou, une soirée est organisée, consacrée à la projection d'extraits des spectacles enregistrés et à celle d'un spectacle en entier. Robert Abirached, directeur du Théâtre et des Spectacles, indique dans son discours introductif que «en attendant la création d'une véritable théâtrothèque, les documents [enregistrés] sont déposés à la Bibliothèque nationale (maison Jean Vilar à Avignon) et ils peuvent être diffusés dans le réseau non-commercial ».

\section{Conclusion}

L'ensemble d'archives administratives que cet article a tenté de décrire, d'archives liées au spectacle et à la mise en scène, versé depuis longtemps aux Archives nationales, est donc très précieux. Il était impossible d'en faire un tour exhaustif dans le cadre d'un article de quelques pages, mais le lecteur aura trouvé des pistes pour lui permettre de poursuivre sa recherche dans des directions qu'il n'aurait peut-être pas envisagées. Ces archives peuvent être aujourd'hui complétées par d'autres fonds. Aux Archives nationales, des versements sont venus s'y adjoindre récemment, qui ouvrent de nouvelles approches. Mais que ce soit dans la conception des bâtiments, dans la mise en scène, dans la réception des spectacles par le public, les préoccupations relatives aux questions de diffusion et d'enregistrement du son, puis de vidéos restent récurrentes. La conservation aujourd'hui aux Archives nationales d'archives audiovisuelles du théâtre de Chaillot et leur mise à disposition du public témoignent de cet intérêt. 


\section{NOTES}

1. Voir, par exemple, Emmanuelle LOYER, Le théâtre citoyen de Jean Vilar, Paris, PUF, 1998 ; Pascal ORY et Pascal BLANCHARD, Chaillot, Palais de la danse, Paris, Somogy, 2018 ; Esprit(s) des lieux. Du Trocadéro au palais de Chaillot, catalogue de l'exposition tenue à la Cité de l'architecture et du patrimoine (17 septembre 2011-17 janvier 2012), Paris, 2011.

2. Voir Marion DENIZOT, «L'engouement pour les archives du spectacle vivant », Écrire l'histoire [En ligne], 13-14 |2014, mis en ligne le 10 octobre 2017, consulté le 12 janvier 2019. URL : http:// journals.openedition.org/elh/475; DOI : 10.4000/elh.475

3. Je remercie Émeline Rotolo, chargée d'études documentaires responsable des fonds relatifs au théâtre et au spectacle vivant à la direction des Fonds des Archives nationales. Elle n'a pas hésité à me faire part de ses recherches et de ses connaissances sur l'histoire de l'administration des théâtres successifs du site de Chaillot. Voir aussi les éléments qu'elle a rédigés sur l'histoire du TNP et du théâtre de Chaillot sur le site des Archives nationales (consulté le 22 décembre 2018).

4. Sous-série F/21.

5. Sous-série F/12.

6. Il concerne la direction du TNP par Jean Vilar de 1951 à 1963. Il est aujourd'hui coté 590AJ. Il comprend plus de 900 cotes. Le fonds a été versé par Jean Vilar.

7. Arch. nat., versement 19900195/1-109.

8. Arch. nat., F/12/11909.

9. Arch. nat., F/21/6143.

10. Arch. nat., $F / 21 / 6143-6155$.

11. Arch. nat., F/21/6145.

12. Théâtre national populaire du Trocadéro, cahier des charges, ministère de l'Instruction publique et des Beaux-Arts, 14 mars 1922, Arch. nat., F/21/6144.

13. Arch. nat., $F / 21 / 5269$.

14. Arch. nat., F/21/3984 et F/21/4681.

15. Arch. nat., F/12/12114.

16. https://www.siv.archives-nationales.culture.gouv.fr/siv/IR/FRAN_IR_056106

17. Arch. nat., F/12/12420.

18. Arch. nat., F/12/12833.

19. Arch. nat., $\mathrm{F} / 12 / 12114$

20. Arch. nat., F/21/6148.

21. Arch. nat., F/12/12184.

22. Arch. nat., F/21/6148.

23. Arch. nat., F/21/6148.

24. Aux Archives nationales, le fonds avait été décrit et classé en différents ensembles cohérents entre 1969 et 2005 par Michel Guillot puis par Claire Sibille et, enfin, par Patrick Laharie. Il a été repris en totalité en 2011 par Magali Lacousse et est désormais facilement accessible aux lecteurs. Il porte les cotes $295 \mathrm{AJ} / 1-948$.

25. Arch. nat., 295AL/259-265.

26. Arch. nat., $295 \mathrm{AJ} / 27-248$.

27. Arch. nat., $295 \mathrm{AJ} / 257$.

28. Arch. nat., $295 \mathrm{AJ} / 367$.

29. Arch. nat., $295 \mathrm{AJ} / 498$.

30. Arch. nat., 295AJ/790-794.

31. Arch. nat., $295 \mathrm{AJ} / 622$.

32. Arch. nat., 295AJ/575-620. 
33. Arch. nat., 295AJ/628-634.

34. Arch. nat., 295AJ/474.

35. Voir l'article d'Émeline Rotolo, « Usages techniques et administratifs de la photographie au sein des archives des théâtres nationaux : du répertoire photographique des décors du théâtre de l'Odéon aux traces d'exploitation des tirages photographiques par les services de communication du TNP », actes du colloque La photographie au théâtre, 23-25 novembre 2017 [à paraître].

36. L'ensemble des photographies est conservé sous les cotes : Arch. nat., 295AJ/848-920.

37. Arch. nat., 295AJ/427 à 436.

38. Arch. nat., $295 \mathrm{AJ} / 770$.

39. Arch. nat., 295AJ/795-839.

40. Arch. nat., 19900195/1-110.

41. Arch. nat., 19900195/109.

42. Arch. nat., 19900195/93.

43. Arch. nat., 19900195/93.

\section{RÉSUMÉS}

Les fonds des Archives nationales permettent de connaître des pans entiers de l'histoire du théâtre de Chaillot, depuis ses origines jusqu'à nos jours. Versés aux Archives nationales pour certains depuis longtemps, ils sont issus de l'administration des Beaux-Arts, de l'administration du théâtre par Jean Vilar, du bureau des théâtres du ministère de la Culture et apportent l'éclairage de l'administration sur l'histoire du théâtre, de sa gestion et de ses spectacles. La présentation de ces archives, variées, fera apparaître des sources qui ont permis d'écrire l'histoire d'un lieu de culture où, avec le temps, tous les arts ont pu être présentés. Elle invitera à poursuivre les recherches avec des points de vue renouvelés.

\section{INDEX}

Mots-clés : Théâtre national populaire, Théâtre national de Chaillot, Trocadéro, administration des Beaux-Arts, architecture, politique culturelle, Wilson (Georges), exposition universelle de 1937, Jean Vilar (Jean), Lang (Jack), Perenetti (Jean-Louis), Savary (Jérôme), Neurdein, Nadar

\section{AUTEUR}

\section{GENEVIÈVE PROFIT}

Responsable du pôle Culture - Beaux-Arts au département de l'Éducation, de la Culture et des Affaires sociales [DECAS], Archives nationales 


\title{
Une mission d'archivage en quête de mémoire, deuxième étape
}

\author{
Justine Dilien
}

\section{Genèse de la mission}

1 Avec le lancement des travaux de la salle Gémier en 2013, le volume important d'archives stockées dans le théâtre avait amené la direction à demander conseil à la Mission des archives du ministère de la Culture et de la Communication. Au cours de l'année 2015, Julie Wannecque, archiviste chargée du suivi de l'archivage pour la direction générale des Médias et de l'Industrie culturelle, le service du Spectacle vivant de la direction générale de la Création artistique ainsi que des établissements publics et opérateurs de l'État attachés (France Médias Monde, France Télévision, Institut national de l'audiovisuel, Radio France, conservatoires nationaux, théâtres et opéras nationaux) avait travaillé avec le théâtre pour le conseiller et évaluer le traitement archivistique à effectuer. Elle en vint à préconiser une mission d'archivage de plusieurs mois au sein du théâtre. En janvier 2016, j'arrivais à Chaillot en tant qu'archiviste chargée de traiter l'arriéré papier, les vidéos analogiques et de mettre en place une politique d'archivage ${ }^{1}$.

\section{Le cadre réglementaire}

2 Le théâtre est devenu établissement public à caractère industriel et commercial (EPIC) par le décret du 21 octobre 1968. À partir de ce moment, les archives produites et reçues ont acquis le statut d'archives publiques, encadrées par la législation en vigueur: le Code du patrimoine et la circulaire du Premier ministre du 2 novembre 2001 relative à la gestion des archives dans les services et établissements publics de l'État. L'établissement a la responsabilité de gérer ses archives au quotidien, mais est soumis au contrôle scientifique et technique (CST) de la Mission des archives du ministère de la Culture. De ce fait, les archives du théâtre constituent une partie du patrimoine national. 


\section{Les missions}

3 Pour permettre la préservation du patrimoine de Chaillot, mes missions comportent plusieurs facettes indispensables. La première est primordiale, puisqu'elle consiste à sensibiliser les agents du théâtre à la bonne gestion de leurs archives.

Les documents qu'ils produisent aujourd'hui dans le cadre de leur activité au théâtre deviendront pour la plupart des archives historiques conservées aux Archives nationales et mises à la disposition du public. Ils comprennent mieux les enjeux des archives, qu'ils soient juridiques, financiers ou historiques. En animant des réunions, en les accompagnant dans leurs pratiques d'archivage et en mettant en place des procédures et outils à leur disposition, ils se sentent plus concernés et prennent conscience qu'ils fabriquent la mémoire de Chaillot. Ainsi, les témoignages de leurs activités seront pérennisés, il n'y aura plus de perte de documents importants. Sans eux, les chercheurs n'auraient pas accès à ces archives et il ne serait pas possible d'exercer le reste de mes missions.

5 La deuxième est la collecte des archives dans les différents services. Chaque service a sa spécificité et permet au théâtre de fonctionner. Il y a d'abord tous les services qui composent la direction technique du théâtre (les régies de scène, de coordination), les services administratifs et les services en relation avec le public. Ils produisent chacun des typologies documentaires à la fois différentes et complémentaires. De plus, chaque personne travaillant dans les services a une histoire propre et un attachement particulier aux archives qu'elle a produites. Une personne ayant fait une carrière de cinq, dix, vingt ans et plus, ne réagira pas de la même manière qu'une personne en début de carrière. Elle souhaitera «trop » conserver ou de ne « rien » conserver. Il est important d'être à l'écoute des gens, de se mettre à leur place et faire preuve de diplomatie. C'est si et seulement si une collecte soignée est faite, qu'un classement ordonné est possible.

6 La troisième est le classement qui va de pair avec l'évaluation, le tri et l'élimination réglementaire. En archivistique, on distingue trois «âges»: l'âge courant, temps de production et d'utilisation des archives; l'âge intermédiaire, temps de conservation pour des raisons juridiques notamment ; l'âge historique, lorsque les archives ne sont plus utilisées par le producteur. Dans ce troisième temps, seules les archives essentielles sont conservées. Une fois que les instruments de recherche sont rédigés, ils permettent aux chercheurs et aux producteurs de retrouver les archives facilement. Dès lors, la communication est possible.

7 Les dernières facettes de ma mission concernent les communications internes au théâtre et les demandes de recherches externes. En effet, orienter les personnes vers les différentes institutions qui conservent des fonds du TNP ou du TNC leur permet d'effectuer leurs recherches plus aisément. La valorisation est l'ultime facette qui permet de faire connaître les archives du lieu et donc la mémoire de celui-ci. 


\section{Les six fonds versés entre janvier 2016 et juillet 2017}

\section{Le fonds des affiches (20160150)}

8 Premier versé en mai 2016, le fonds comporte des affiches produites par le service de la communication entre 1988 et 2011. Au nombre de 254 affiches cotées pour 559 exemplaires, 13 dessins et 49 épreuves, elles sont majoritairement de dimensions 40 x $60 \mathrm{~cm}$. Le fonds recouvre les trois directions de Jérôme Savary (1988-2000), Ariel Goldenberg (2000-2008) et Dominique Hervieu (2008-2011). L'arrivée d'une nouvelle direction s'accompagne souvent d'un désir de nouveauté qui passe par le changement de la charte graphique par des agences ou des designers.

Durant les douze saisons de la direction de Jérôme Savary, des affiches-programmes étaient mises à la disposition du public. Ainsi, pour un spectacle, il y avait au recto une photographie ou un dessin représentant ou non les acteurs, majoritairement en nuances de gris avec quelques touches de couleur (bleu, rouge et jaune), superposé par le texte de la distribution. Et au verso, il y avait la partie programme avec le texte expliquant le spectacle. C'est l'agence «Crapule ! Productions » qui réalisa le graphisme des quatre premières saisons. À partir de la saison 1992-1993 jusqu'à la fin de sa direction, c'est l'agence « M ! Frédéric Mei » qui se chargea du graphisme.

Pour les huit saisons sous la direction d'Ariel Goldenberg, il a été décidé de revenir à une affiche ne comportant qu'un recto. Cependant, pour les trois premiers spectacles de la saison 2000-2001, s'étalant du 14 septembre au 23 décembre 2000, le graphisme de « M ! Frédéric Mei » a été conservé. Cela s'expliquait par le fait que les trois spectacles « Top Dogs », «L'amant-La collection » et « Un trait parfait » ont été programmés par la direction de Jérôme Savary. Ainsi, la programmation de Goldenberg ne commence qu'en janvier 2001. C'est la graphiste Corinne Thévenon qui réalisa les affiches de janvier à juin 2001, avec une charte graphique sombre, excepté pour le spectacle de «Le jardin io io ito ito » de José Montalvo et Dominique Hervieu. À partir du festival «Buenos Aires Tango » de mai 2001, jusqu'à la fin de la direction de Goldenberg (2008), c'est le graphiste Michal Batory qui impose un nouveau concept. La couleur prédomine et des objets, animaux ou nourritures sont détournés pour illustrer les spectacles.

11 Les trois saisons de la directrice Dominique Hervieu, habituée de Chaillot, par ses spectacles de la compagnie Montalvo-Hervieu, sous la précédente direction, et par sa fonction d'ancienne directrice de la mission jeune public depuis le début du XXI ${ }^{\mathrm{e}}$ siècle, ont imposé une prédominance de danse au théâtre. Les affiches des quatre spectacles du premier semestre de la saison 2008-2009 sont encore réalisées par Michal Batory. À partir de janvier 2009, à la fin de sa direction, c'est l'agence «La vache noire » qui a la charge de la conception des visuels. Les couleurs vives et les photographies de danseurs caractérisent ses affiches.

Seuls deux intrus se sont glissés dans ce fonds d'affiches de ces trois directions. Une affiche de la saison 1988-1989 portant le logo de la direction d'Antoine Vitez, qui a dû être imprimée avant la nomination de Jérôme Savary. L'affiche du spectacle «iD » du cirque Éloize, de la première saison de la direction de Didier Deschamps a également été versée, en raison des mêmes dimensions et graphisme utilisés par la direction précédente. Des épreuves d'affiches (aussi appelées cromalins) et des dessins permettent de mieux comprendre les étapes de la création visuelle sous la direction de Jérôme Savary. 
Figure 1

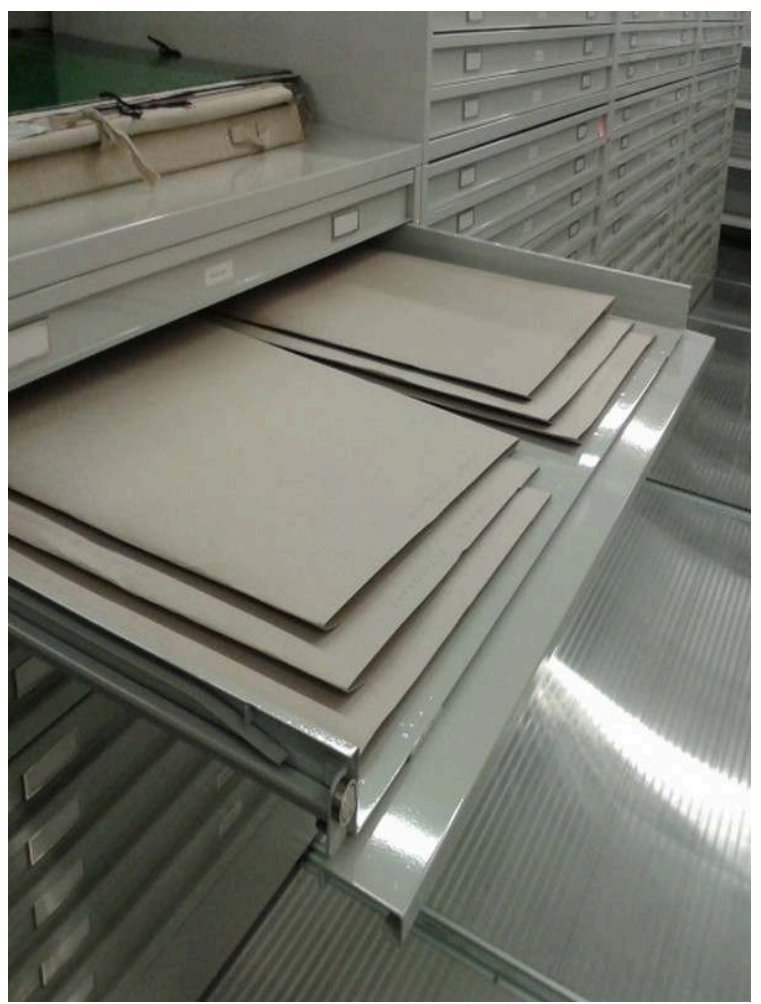

Conditionnement des affiches aux Archives nationales, site de Pierrefitte-sur-Seine Photographie : Nadine Gastaldi

\section{Les fonds audiovisuel (20160438) et sonore (20170371)}

La première partie du classement des fonds jusqu'à leur versement aux Archives nationales en 2016 et 2017 a été réalisée au sein de la régie audiovisuelle du théâtre. Il a consisté à récoler les vidéos et sons sur supports analogiques et numériques présents dans cinq armoires, et à les décrire dans l'instrument de recherche. Sachant qu'il était impossible de lire les vidéos et sons sur supports physiques, les descriptions ont été faites avec les indications figurant sur les boîtes et les recherches documentaires effectuées. La préparation du conditionnement et du transport des 1821 supports physiques des archives audiovisuelles, puis des 368 supports physiques d'archives sonores a été faite avec l'aide d'Olivier Valat et Franck Oslé, techniciens du département audiovisuel des Archives nationales. Les 576 vidéos numériques ont été transférées sur des disques durs. C'est Sandrine Gill qui a repris le traitement des fonds aux Archives nationales, permettant l'affinement du classement et des descriptions qui n'étaient parfois que des hypothèses.

\section{Le fonds habillement (20160646)}

Ce fonds fut versé en mai 2017 et comporte des dossiers de 69 spectacles et un événement hors-programmation. Il s'agit du centenaire du monogramme de la toile Louis Vuitton, manifestation pour laquelle l'entreprise a loué le grand foyer du théâtre et engagé Jérôme Savary pour réaliser le spectacle. Il a été produit par le service 
habillement-couture du théâtre entre 1988 et 2008. Il couvre les trois directions d'Antoine Vitez, de Jérôme Savary et d'Ariel Goldenberg. C'est un petit fonds (5 dimabs et 1 portefeuille pour 1,65 mètre linéaire), mais il est d'une grande richesse. En effet, les dossiers sont constitués de conduites d'habillement, d'inventaires de costumes essentiellement manuscrits, de morceaux de tissus, de dessins de costumes originaux ou non et de photographies majoritairement prises par le service lors d'essayages ou de création de costumes. Les dessins originaux conservés dans ce fonds sont ceux du Mariage de Figaro ou la folle journée, de Patrice Cauchetier, spectacle joué du 5 février au 4 avril 1987, et de Mambo Mistico de Françoise Tournafond, du 19 mars au 23 avril 2005. On retrouve aussi des photographies des marionnettes du Soulier de satin créées par le service à l'image des personnages de la pièce et dont chacune fut cousue par une couturière-habilleuse. Ces archives ont été essentiellement constituées et conservées par Nicolle Daviot, cheffe habilleuse-couturière depuis mai 2011, mais arrivée au théâtre en novembre 1967. En 51 ans de carrière, elle a connu huit directions (de Georges Wilson à Didier Deschamps) et ce fonds reflète en partie la passion de son travail d'habilleuse-couturière.

Figure 2

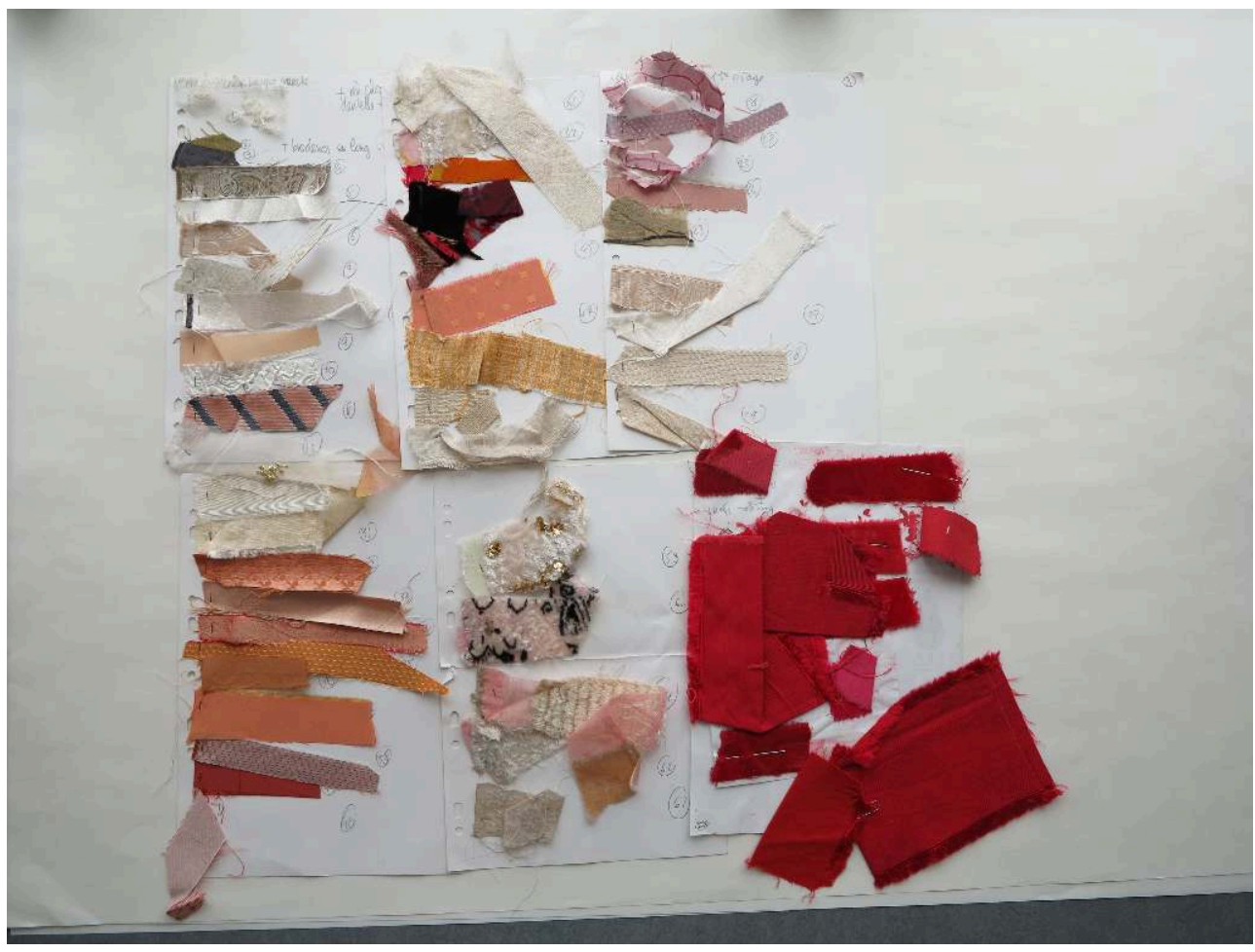

Morceaux de tissus du « Bourgeois Gentilhomme » mis en scène en 1996 (Arch. nat. 20160646/6)

\section{Le fonds de la direction de Jérôme Savary, 1988-2000 (20160552)}

Il fut versé en mai 2017 et concerne les archives papier de tous les services du théâtre, excepté celles du service de l'habillement et des régies audiovisuelles et sonores qui ont leurs propres fonds. Il s'étend de 1975 à 2003 et se compose de 82 cartons d'archives (Dimab) et de 6 registres de comptes (27,4 mètres linéaires). Il nous permet de retracer à la fois l'histoire sociale et artistique du théâtre sous sa direction. La difficulté de 
classement de ce fonds fut de séparer les archives privées des archives publiques. Directeur de l'établissement théâtral public, comédien et metteur en scène pour les spectacles programmés à Chaillot, Jérôme Savary continua à monter des spectacles dans d'autres lieux privés, mélangeant les deux sphères à Chaillot. Des archives dites «artistiques» de spectacles comme La femme du boulanger de Marcel Pagnol avec Michel Galabru, joué en 1985, ont été retrouvées à Chaillot, ou encore La légende de Jimmy de Michel Berger et Luc Plamandon monté en 1990 au théâtre Mogador. Elles ont été extraites du fonds d'archives publiques.

Dans ce fonds, on apprend aussi que l'école voulue par Antoine Vitez au sein du théâtre fut reprise sous Jérôme Savary et renouvelée à son image avec l'introduction de cours de comédie musicale. Chaillot était aussi un lieu d'accueil d'événements extérieurs, telle que la cérémonie officielle de la commémoration du bicentenaire de la Révolution française le 13 juillet 1989, des défilés de mode comme Hugo Boss le 5 juillet 1995, un plateau de télévision pour le passage à l'an 2000, des expositions en l'honneur d'artistes ou des directions précédentes sur Gérard Philipe en 1989, Jean Vilar en 1991 et Antoine Vitez en 1994. Ce lieu pluridisciplinaire perpétuait ainsi la mémoire populaire de ses prédécesseurs. On trouve des "perles", des lettres envoyées par des spectateurs au directeur. Ainsi, dans le chrono de Jérôme Savary (20160552/1), on peut lire sous la plume d'un spectateur étant allé voir D'Artagnan en 1988 qu'il a « l'impression de vivre l'action en même temps, et presque à la place des personnages", et qu'il «se sent comme traversant la Manche, ainsi que galopant sur un cheval ». On peut s'attarder sur des plans (20160552/166) de la tournette du plateau du spectacle d'Irma la douce en 2000, dont l'ancien emplacement est encore visible sous la scène de la salle Jean-Vilar.

Figure 3

Figure 3

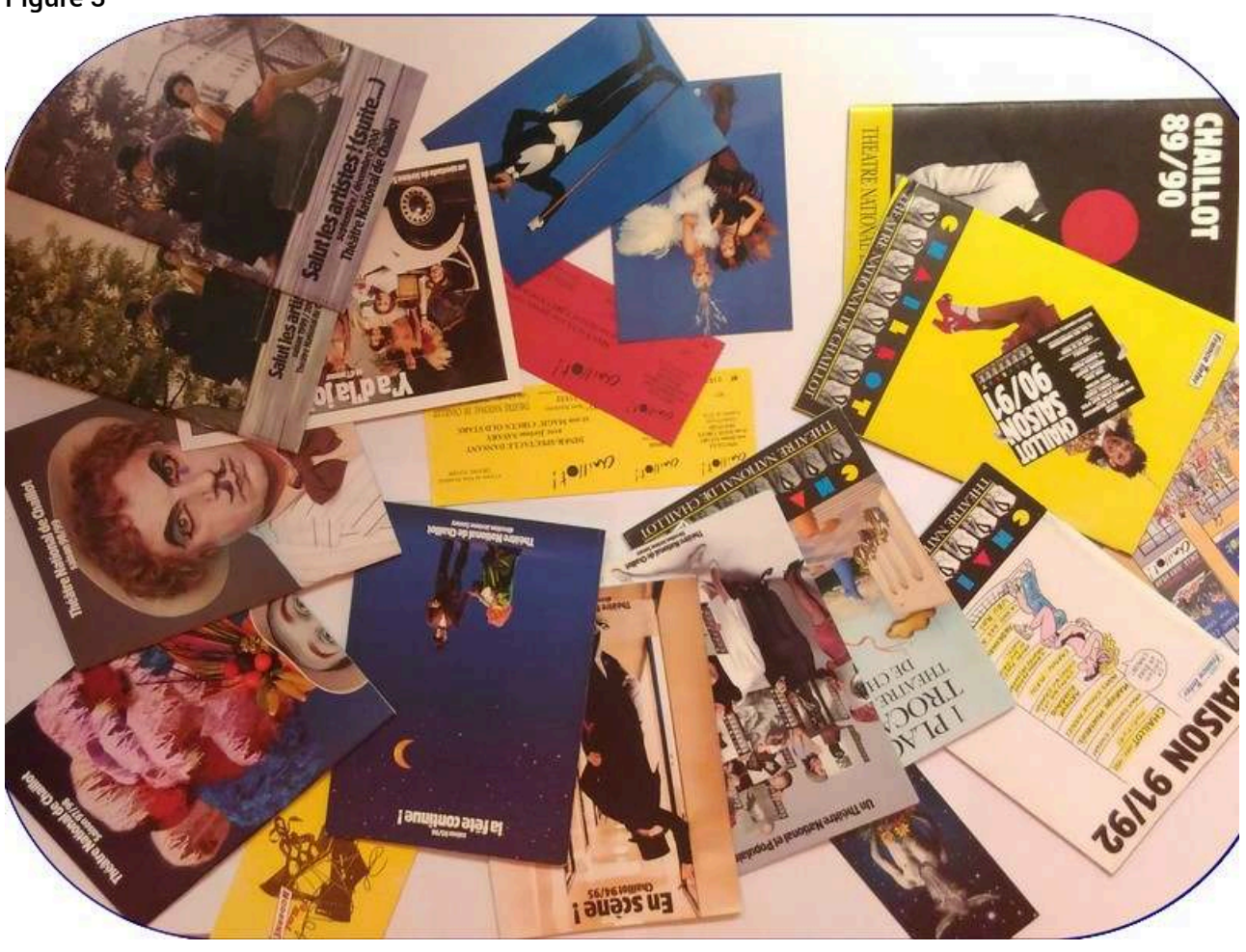

Pêle-mêle de programmes de saisons, visuels et billets d'entrée de la direction Jérôme Savary 
Figure 4

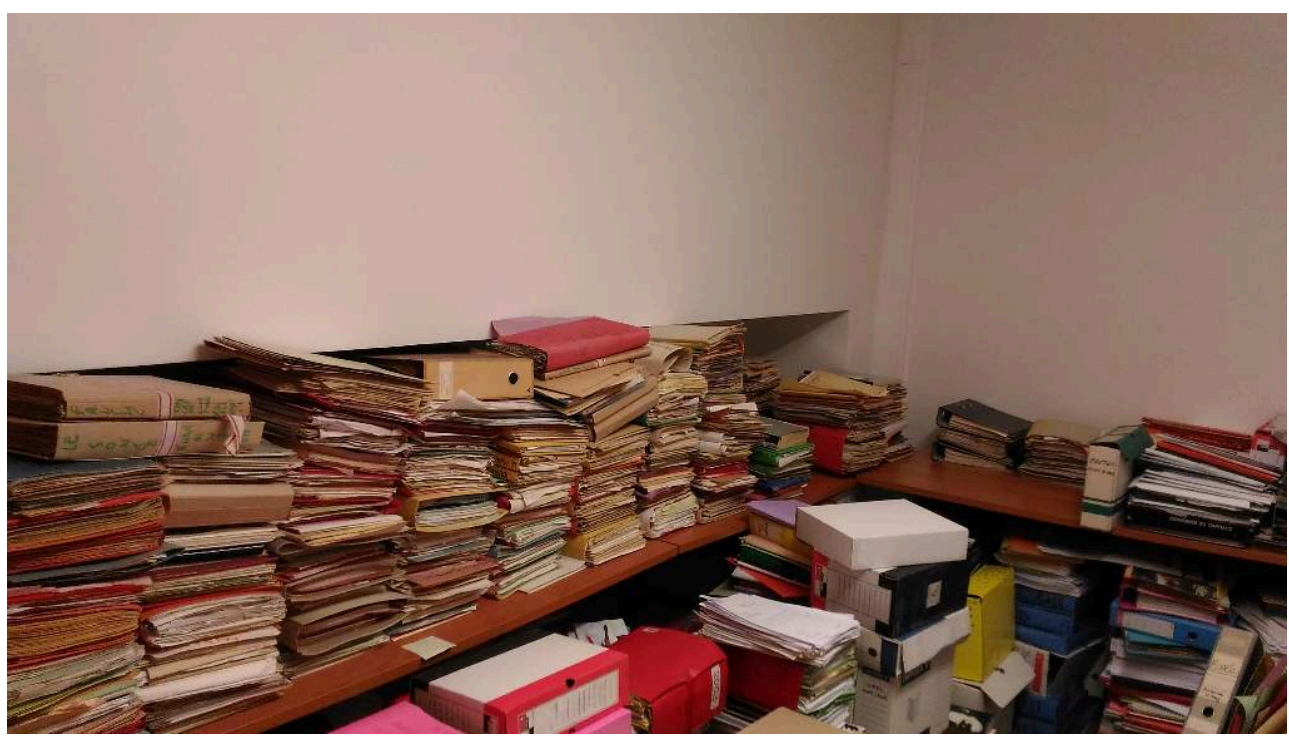

Avant le classement du fonds Savary

Figure 5

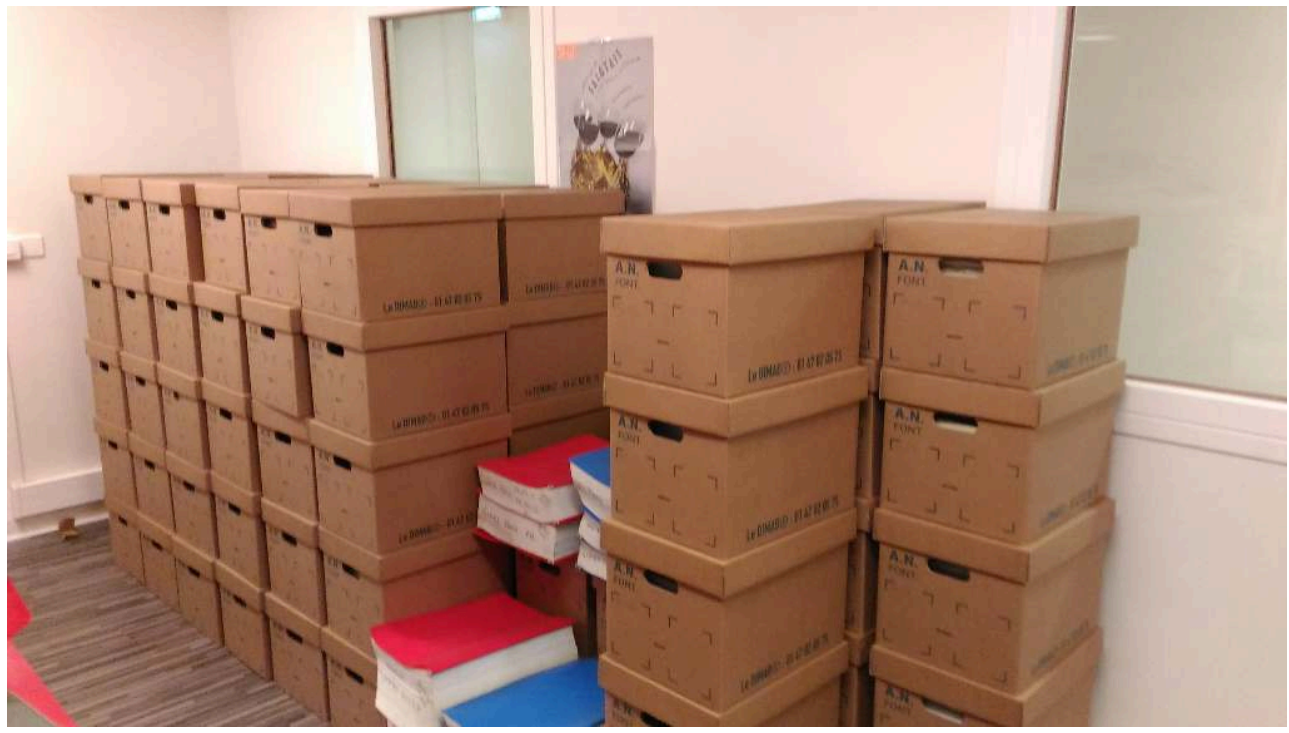

Après le classement du fonds Savary

\section{Le fonds des directions de Jean Vilar (1951-1963), Georges Wilson (1963-1972), Jack Lang (1972-1974), André-Louis Perinetti (1975-1981), Antoine Vitez (1981-1988) [20170180]}

17 Versé en novembre 2017, ce fonds complémentaire de ceux déjà existants aux Archives nationales (295 AJ et 19900195) et à la Bibliothèque nationale de France, se compose de 57 dimabs et 19 planches de photographies collées (17,1 mètres linéaires). Il contient, comme celui de la direction de Jérôme Savary, les archives papier de tous les services, excepté celles du service de l'habillement-couture et des régies audiovisuelle et sonore. 
Restées au théâtre, elles n'avaient pas encore fait l'objet de versement. Ce fonds s'étale de 1941 à 1996 et contient majoritairement des documents administratifs pour la période de Jean Vilar à Georges Wilson. Les archives dites "artistiques" sont plus nombreuses sous la direction d'Antoine Vitez et des dossiers incomplets des spectacles sous les autres directions s'y ajoutent. La date de 1941, antérieure à la direction de Jean Vilar, s'explique par la présence de ce projet de convention collective sur du papier pelure, non signé et en mauvais état, dans un dossier de révision de la convention collective de 1973. Dans ce fonds, on apprend que Chaillot fut un lieu de cinéma d'art et d'essai, avec des présentations de films sélectionnés au Festival de Cannes, de "Chansons au TNP» accueillant des artistes populaires tels que Juliette Greco et Georges Brassens, mais aussi de danse, invitant Maurice Béjart et son Ballet du $\mathrm{xx}^{\mathrm{e}}$ siècle à plusieurs reprises. Mais on comprend aussi l'histoire de la création de l'école, la gestion des événements locatifs gérés par l'administrateur des galas et l'aménagement du théâtre. Antoine Vitez, caméléon dans les murs de Chaillot, se transforme au gré de ses envies en comédien, metteur en scène, poète ou encore enseignant, tout en restant à la direction du théâtre. Les dossiers des spectacles prouvent la diversité des genres artistiques programmés. En consultant ce fonds, on découvrira des trésors gardés cachés dans les méandres du lieu, comme des contrats dactylographiés et manuscrits originaux ou des conventions d'abonnements avec la Société des auteurs et compositeurs dramatiques (SACD) signés et paraphés par Jean Vilar, puis par Georges Wilson (20170180/66 et 67). Il comprend également trois registres comptables manuscrits datant de 1959 à 1984 (20170180/72) de l'administrateur des galas et les dossiers de carrière des directeurs André-Louis Perinetti et Antoine Vitez.

Figure 6

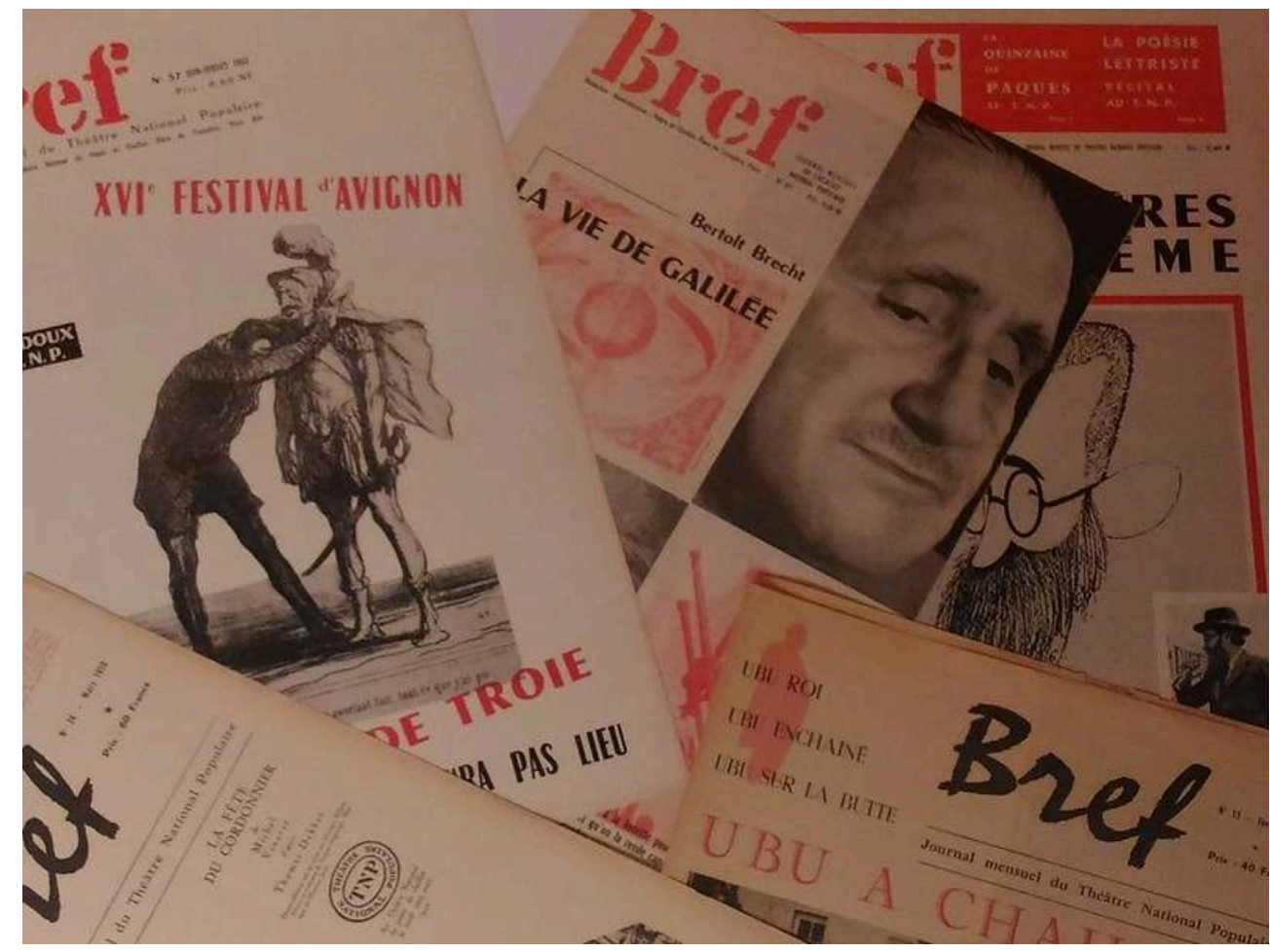

Pêle-mêle des « Bref » sous la direction de Jean Vilar et Georges Wilson 
Figure 7

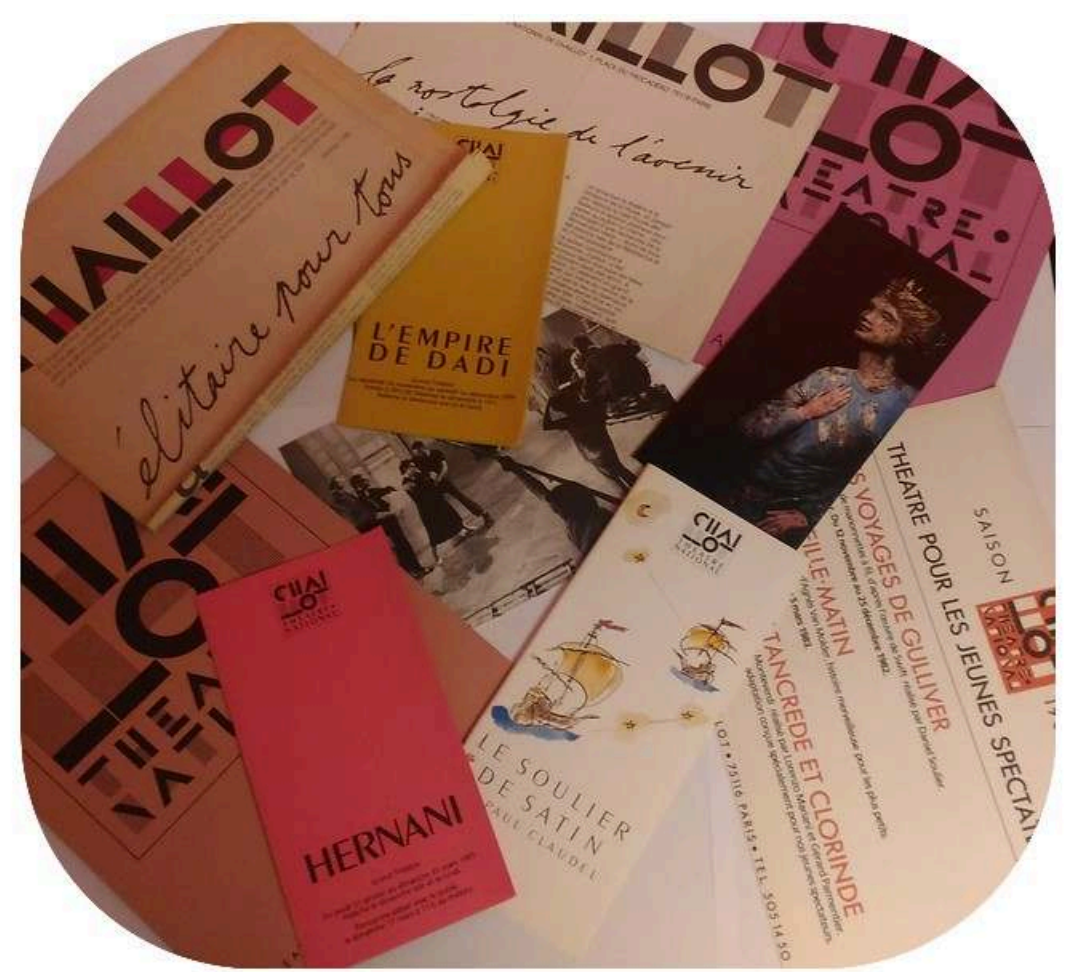

Pêle-mêle des visuels sous la direction d'Antoine Vitez

Figure 8

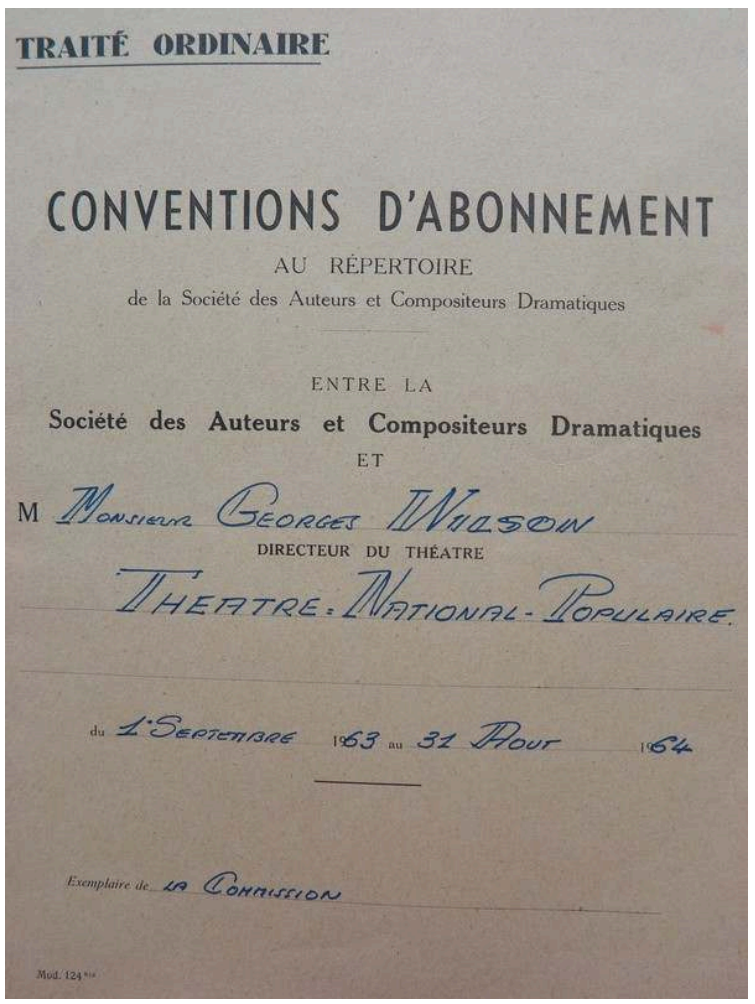

Contrats avec la SACD (Arch. nat. 20170180/66) 
Figure 9
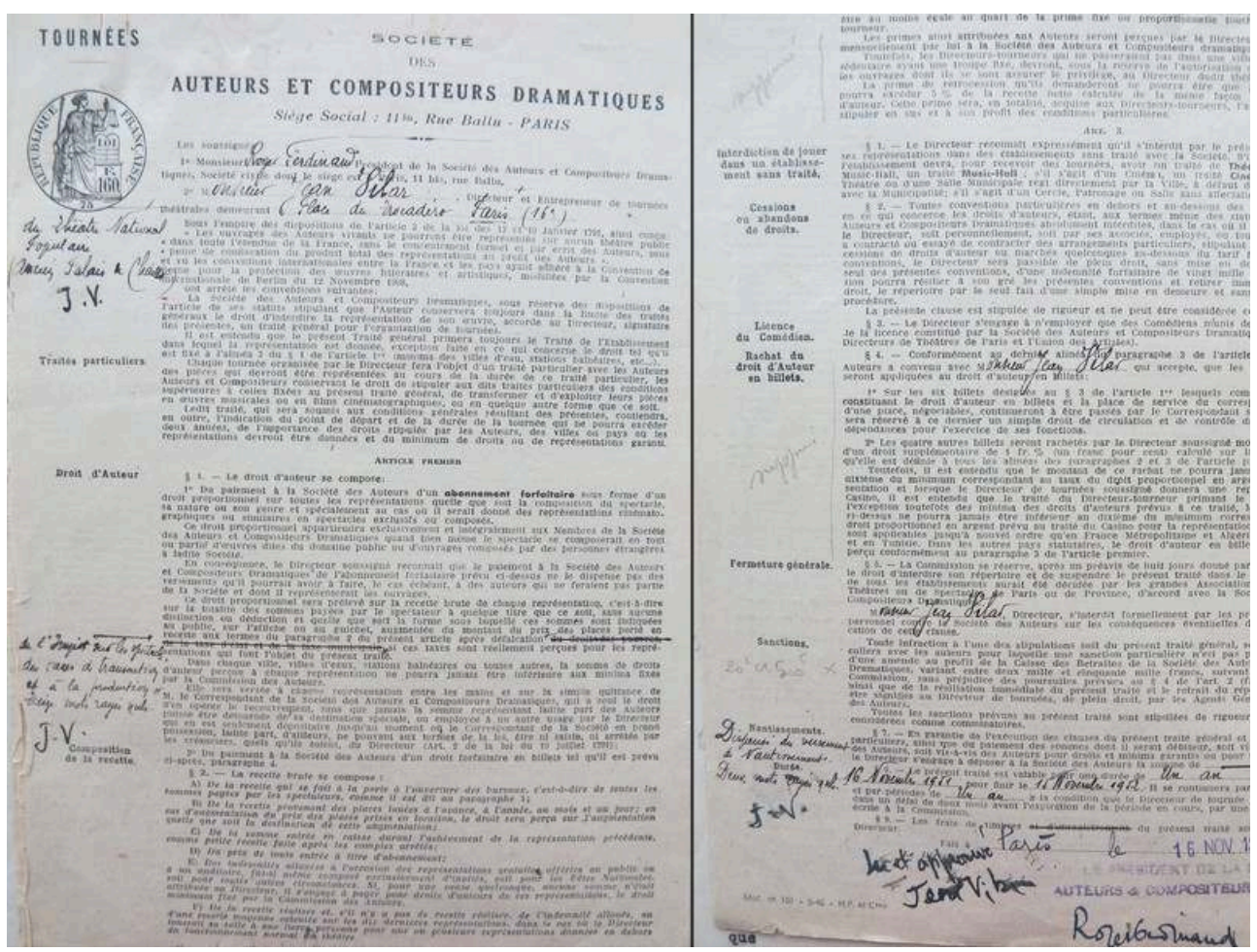

Contrats avec la SACD (Arch. nat. 20170180/ 67)

Figure 10

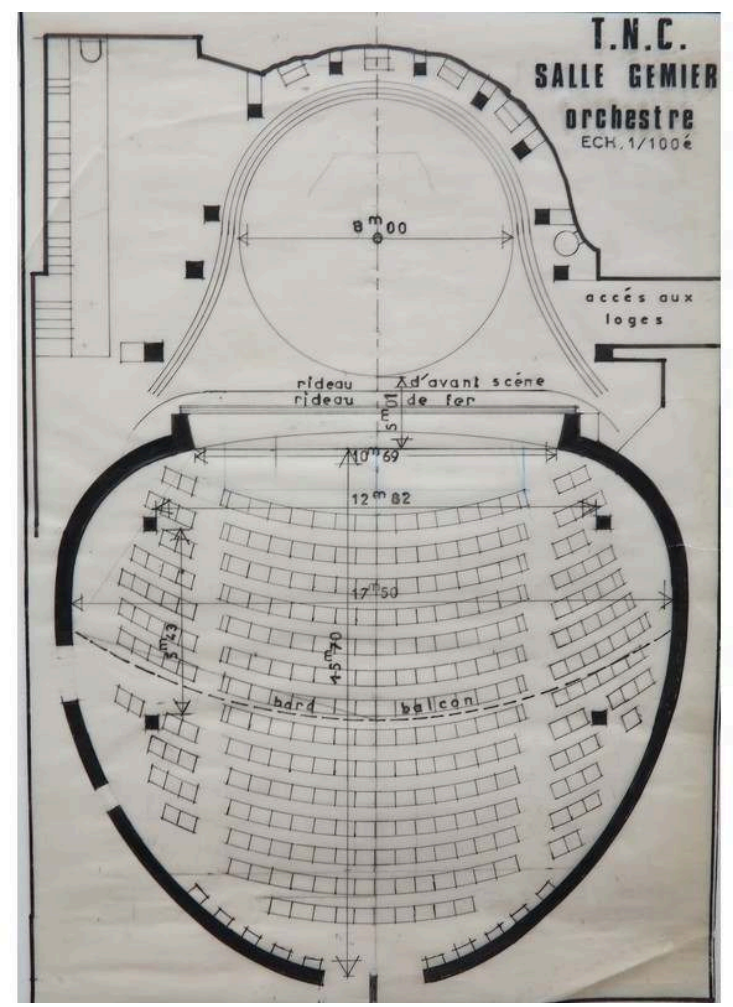

Plans de la salle Gémier (Arch. nat., 20170180/79) 
Figure 11

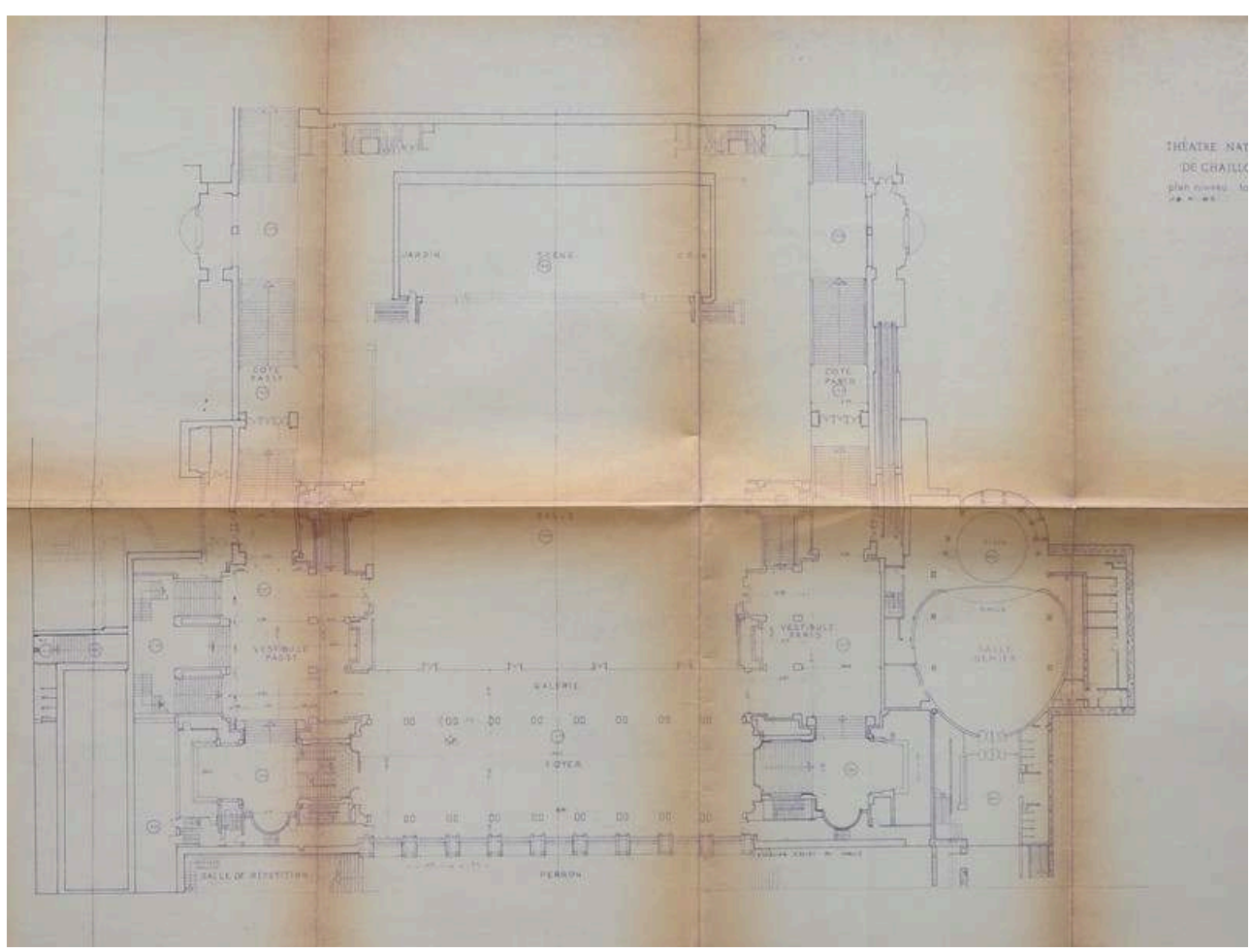

Vue d'ensemble du théâtre de 1985 (Arch. nat., 20170180/79)

Figure 12

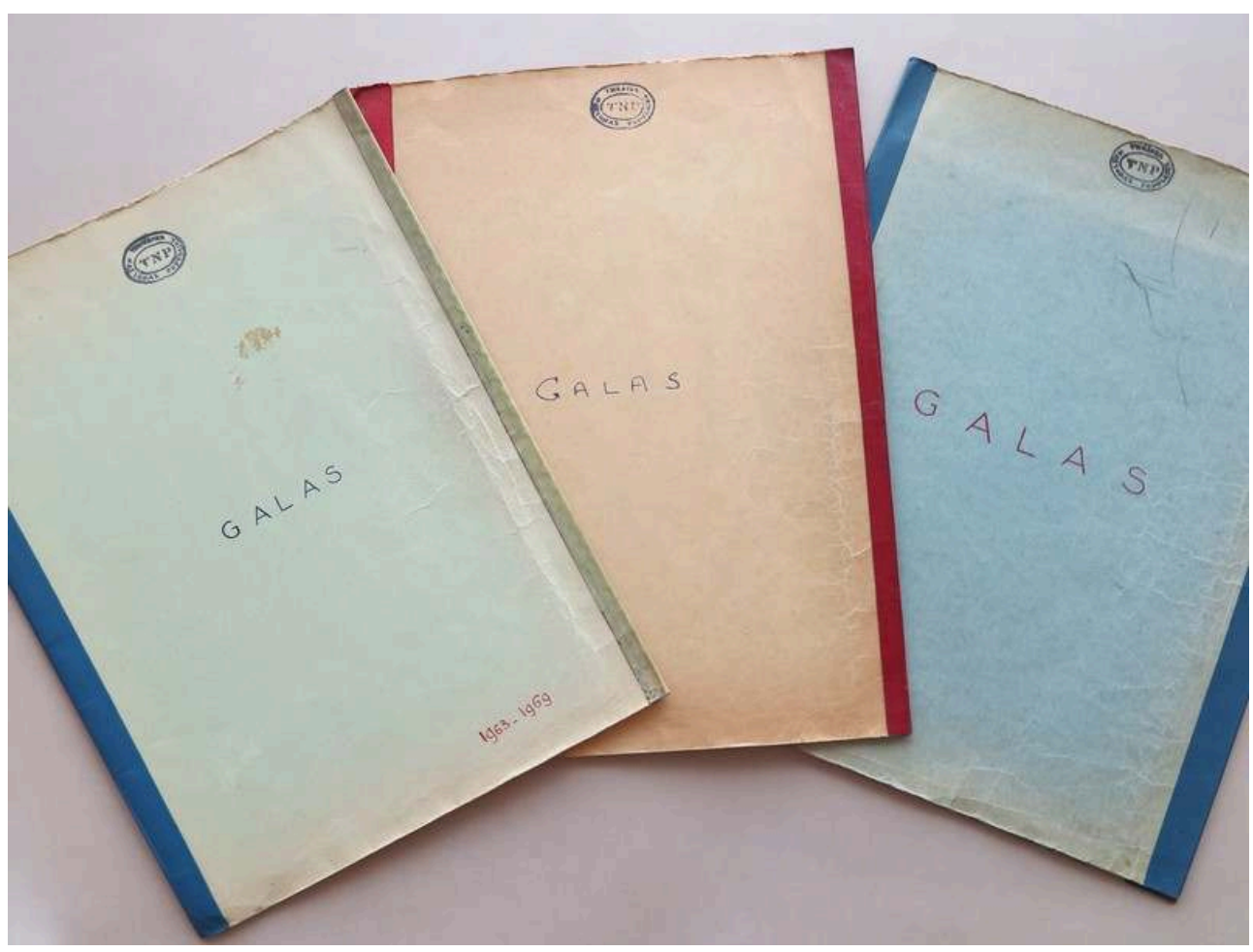

Trois registres de l'administrateur des galas (Arch. nat., 20170180/72) 


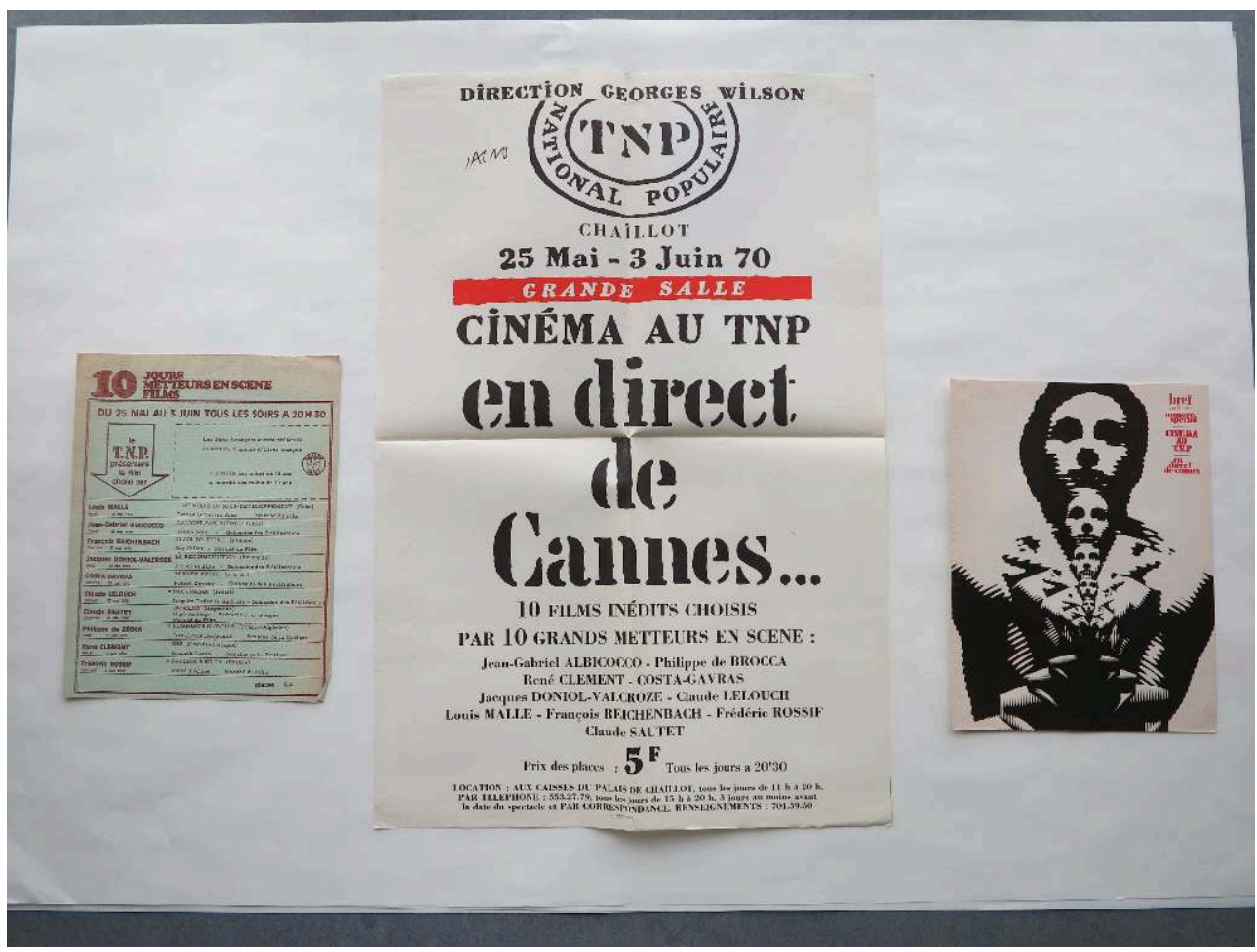

"Cinéma au TNP », « Dix metteurs en scène, dix films » présentés au festival de Cannes du 25 mai au 3 juin 1970 à la grande salle (Arch. nat., 20170180/86)

\section{Conclusion}

Cette mission de dix-huit mois passée au sein du théâtre m'a permis de sensibiliser le personnel à l'histoire qu'il fabrique et de mettre au jour de multiples documents sur des supports variés. Ces archives retracent quarante-neuf ans de mémoire de ce lieu dédié à tous les arts et permettent de suivre l'évolution sociale et artistique de six directions. Revenue à Chaillot de mars à septembre 2018, j'ai pu classer le fonds de la direction Ariel Goldenberg, directeur de 2000 à 2008, et un fonds photographique révélant de magnifiques photographies des spectacles joués principalement sous la direction de Jérôme Savary. Les archives de cet établissement unique, acteur de l'histoire artistique française, sont aujourd'hui partagées avec un très large public international. Elles portent bien leur nom : « élitaire pour tous ».

\section{NOTES}

1. Je remercie la direction de Chaillot - Théâtre national de la Danse, Didier Deschamps, Réda Soufi et Benoît André, pour la volonté d'effectuer ce chantier d'archivage, jamais 
réalisé auparavant, ainsi que mes collègues de Chaillot - Théâtre national de la Danse et, plus particulièrement, Nicolle Daviot, pour leur accueil et gentillesse à mon égard, et sans qui les archives n'existeraient pas. Ma collègue Julie Wannecque, de la Mission des archives du ministère de la Culture, m'a apporté son soutien et ses conseils aguerris durant toute la durée de la mission. J'ai également bénéficié des conseils de Martine Sin Blima-Barru et Sandrine Gill du département de l'Archivage électronique et des Archives audiovisuelles des Archives nationales sur les questions des archives audiovisuelles et sonores. Je suis également reconnaissante envers Émeline Rotolo et Geneviève Profit du département de l'Éducation, de la Culture et des Affaires sociales des Archives nationales, pour leur aide sur le choix des photographies des archives présentées.

\section{RÉSUMÉS}

Entre 2016 et 2018, une mission d'archivage a eu lieu au théâtre national de Chaillot. De la collecte à la communication en passant par la sensibilisation au sein des services, la mission revêt plusieurs facettes pour permettre la préservation du patrimoine de Chaillot. Entre mai 2016 et novembre 2017, quatre fonds papiers (20160150: fonds des affiches, 20160646 : fonds du service habillement, 20160552 : fonds de la direction de Jérôme Savary, 20170180 : fonds des directions Vilar à Vitez) ont été versés aux Archives nationales, parallèlement aux fonds des archives audiovisuelles et sonores.

\section{INDEX}

Mots-clés : Théâtre national populaire, Théâtre national de Chaillot, Trocadéro, Vilar (Jean), Vitez (Antoine), Perenetti (Jean-Louis), Wilson (Georges), Lang (Jack), Savary (Jérôme), Goldenberg (Ariel), Hervieu (Dominique)

\section{AUTEUR}

\section{JUSTINE DILIEN}

Archiviste à Chaillot-Théâtre national de la Danse 


\title{
La mémoire des spectacles du Trocadéro et de Chaillot au département des Arts du spectacle de la Bibliothèque nationale de
} France

\author{
Joël Huthwohl
}

1 Le département des Arts du spectacle de la Bibliothèque nationale de France a pour mission de collecter, conserver, signaler et valoriser la mémoire du spectacle vivant en France. À la fois bibliothèque, centre de documentation, service d'archives et musée, il détient la plus importante collection dans ce domaine en France et une des premières en Europe. L'origine du département remonte à la collection du banquier, polytechnicien et passionné de spectacles Auguste Rondel (1858-1934) qui a fait don à l'État en 1920 de plus de quatre cent mille documents de toute nature sur les arts du spectacle. Ce cœur patrimonial fondateur a continué à être enrichi au fil des décennies et jusqu'à aujourd'hui par tout type de documents et objets gardant la trace de l'histoire des artistes et des lieux de spectacle en France. Les Collections théâtrales ont donné naissance en 1976 au département des Arts du spectacle, désormais installé sur le site Richelieu de la BnF. Dans ce vaste ensemble de plus de 20 kilomètres linéaires, on compte plus de 350 fonds d'archives, depuis celles de théâtres parisiens du xix siècle comme le Théâtre du Palais-Royal à celles de femmes et d'hommes de spectacle plus contemporains comme Eugène Ionesco, Ariane Mnouchkine ou encore Jacques Lassalle. Ce sont des ensembles multi-supports qui réunissent des manuscrits de pièce, des correspondances, des relevés de mises en scène, des documents techniques et administratifs, des maquettes de décors et de costumes, des programmes et affiches, de la presse, des documents audiovisuels, des costumes et accessoires, tout ce qui fait le patrimoine du spectacle vivant.

2 Dans ces collections, les théâtres du Palais du Trocadéro puis du Palais de Chaillot ont naturellement leur place en matière de documentation et d'iconographie comme en 
matière d'archives. La cartographie globale n'est pas facile à dresser car les ramifications sont nombreuses. Certains ensembles sont directement identifiés comme se rapportant à ces lieux et à leur histoire, mais leurs ressources n'épuisent pas les possibilités d'exploration. On peut par exemple penser au dossier iconographique de la danseuse de flamenco Argentina, qui s'est produite au Trocadéro au début des années 1930, ou au fonds d'Armand Gatti pour les manuscrits de Chant public devant deux chaises électriques, créé à Chaillot en 1966 et de la Passion du général Franco, pièce programmée en 1968 et censurée, ou encore au fonds Gérard Philipe pour l'enregistrement sonore des répétitions de Nucléa de Henri Pichette qui a fait l'ouverture du TNP en 1952. Il est donc difficile d'être exhaustif sur le sujet. Une présentation historique et méthodique peut toutefois permettre de mesurer l'étendue des richesses documentaires du département et aider les chercheurs à faire un premier repérage.

\section{Le temps du Trocadéro}

Dans la collection Auguste Rondel, dans la série RT-histoire du théâtre ${ }^{1}$, sont réunis les programmes du Palais du Trocadéro à partir de 1896 auxquels sont jointes, selon la pratique du collectionneur, les coupures de presse ayant trait aux événements représentés. Cette série, discontinue dans les premières années, devient très complète à partir de 1920 et de la création du Théâtre national populaire. Elle forme une épine dorsale à laquelle peuvent se rattacher de nombreux autres dossiers documentaires sur des personnalités ayant marqué l'histoire du lieu, notamment les metteurs en scène et les acteurs dans la série RT et les danseurs et chorégraphes dans la série RO. Pour ces artistes, même méconnus, les dossiers iconographiques de la série ICO-PERIconographie personnalités fournissent des photographies et des défets de presse précieux. En complément, on peut consulter la série WNA, sous la cote WNA-21 pour le Trocadéro, qui commence en 1907 et a été constituée de programmes venant du dépôt légal ou de dons plus récents. Pour cette période du Trocadéro, on mentionnera aussi deux maquettes d'Émile Bertin ${ }^{2}$ : l'une en particulier est un dessin à la plume et lavis pour le 11 novembre 1920, date de l'inauguration du TNP, représentant le podium avec le fond de scène orné de drapeaux. Enfin deux fonds d'archives peuvent permettre un approfondissement, celui de Mario Meunier ${ }^{3}$, secrétaire d'Isadora Duncan, qui fut la première danseuse moderne à se produire au Trocadéro en 1913, et de celui d'Argentina ${ }^{4}$.

\section{Le Théâtre national de Chaillot : une documentation et une iconographie abondantes}

4 Après la mort d'Auguste Rondel en 1934, la constitution d'une mémoire documentaire sur les spectacles en France se poursuit. Une nouvelle série nommée Rsupp-Rondel supplément s'ouvre en 1938, l'année précédant l'ouverture du nouveau Palais de Chaillot. Les recueils de coupures de presse et de programmes du Rsupp sont aujourd'hui numérisés et accessibles dans la bibliothèque numérique BnF-Gallica ou Gallica intra-muros pour les années postérieures à 1946. L'internaute y trouve par exemple deux recueils sur l'inauguration du 10 mars $1939^{5}$ qui remontent en réalité aux travaux de 1937, ainsi que de nombreux articles sur les pièces, ballets et films au programme du théâtre. De 1962 à 1977, la presse et les programmes sont réunis sous la 
cote SW. En parallèle dans la série WNA, sous la cote WNA-21, on trouve une série très complète des programmes, tracts et dossiers de presse de 1938 à aujourd'hui. En résumé, la $\mathrm{BnF}$ possède sans doute la série la plus complète des programmes de Chaillot, généralement associée à des coupures de presse avant 1977. Toutefois, étant donné le nombre important de soirées uniques, notamment les soirées de gala, qui marquent l'histoire du lieu, la collection ne saurait prétendre à l'exhaustivité.

5 La BnF a aussi pour mission de collecter les sites Internet du domaine français. Le " dépôt légal du Web » inclut le monde du spectacle et accompagne ainsi la mutation de la communication des théâtres et des compagnies du papier vers le numérique. Pour Chaillot, la collecte est significative à partir de la saison 2004-2005. Elle est faite plusieurs fois par an ; elle donne ainsi une idée de l'évolution du site et permet d'avoir accès à un grand nombre d'informations sur la vie sur l'actualité du théâtre. Les Archives de l'Internet sont accessibles sur place seulement pour des raisons juridiques liées au respect du droit d'auteur.

En matière d'iconographie, la série ICO-PER, comme pour la période précédente, est riche de portraits en scène ou à la ville des artistes s'étant produits à Chaillot. Par ailleurs, on trouve quelques documents dans la série ICO-THE-scènes de théâtre ${ }^{6}$ avec, par exemple, la photographie plutôt rare d'une lecture, celle de Clovis de France de Max Frantel en 1944 par l'auteur lui-même. De même, la série ICO-ARC-architecture des théâtres ${ }^{7}$ contribue au propos, modestement même si elle réserve une belle surprise avec un tirage de Robert Doisneau immortalisant l'équipe du théâtre sur le plateau autour de Jean Vilar en 1952. Beaucoup plus riches sont les fonds de photographes notamment Bernand, Roger Pic, Daniel Cande et Brigitte Enguérand, qui, chacun à leur époque, ont suivi les spectacles du théâtre. Une partie de ces clichés sont numérisés et accessibles dans la BnF-Gallica. On trouve par exemple près de 80 spectacles photographiés par Daniel Cande de Par les villages de Peter Handke, mis en scène par Claude Régy en 1983, à Nina Stromboli, texte et mise en scène de Jérôme Savary en 1996. La collection de maquettes de décors et de costumes est elle aussi particulièrement intéressante. Elle recèle plus de 80 dessins originaux, depuis les maquettes d'André Boll pour les Molière de 1939 jusqu'aux maquettes de Roland Topor pour Ubu roi en 1992, en passant par les œuvres d'Édouard Pignon pour Mère Courage, celles de Hubert Monloup pour Chant public pour deux chaises électriques d'Armand Gatti, celle d'Ezio Frigerio pour Richard II ou encore de Yannis Kokkos pour Hamlet et La Mouette, sans compter les dizaines de maquettes de Jacques Le Marquet que nous retrouverons plus loin. Notons enfin trois maquettes d'affiches de Maurice Jacno pour des spectacles de 1971-1972. Les affiches elles-mêmes sont conservées en nombre significatif, grâce au dépôt légal et à la collecte régulière et couvrent toute la période de l'après-guerre.

7 Il serait paradoxal d'oublier, avant de passer au domaine des archives, celui de la bibliothèque au sens strict, autrement dit des livres et des revues. Sont à la disposition des lecteurs les textes de pièces - en particulier la fameuse collection du répertoire du TNP -, les ouvrages historiques sur le Trocadéro et Chaillot, les mémoires de metteurs en scène ou d'acteurs, les articles et témoignages depuis les origines du théâtre jusqu'à aujourd'hui puisque les collections s'enrichissent au fil des nouvelles publications. 


\section{Les fonds d'archives}

8 spectacle conserve une partie des archives du Théâtre de Chaillot, principalement pour la période 1963 à 1997.

Les archives pour la période antérieure à 1963 sont peu volumineuses. Elles émanent d'un don de 1997 fait par le service de documentation du théâtre dirigé alors par Jacques Roullet. On y trouve quelques documents sur les spectacles de la direction de Firmin Gémier (1920-1933) : programmes, presse et photographies. Pour les directions de Paul Abram (1938-1940) et Pierre Aldebert (1940-1951), il s'agit, outre quelques documents sur le bâtiment entre 1934 et 1937, d'une série de dossiers sur les décors des spectacles de cette période - plantations et photographies de décors. Enfin on trouve dans cet ensemble le «livre de bord» du théâtre de 1939 à 1953. C'est le registre journalier des représentations. Le dernier registre prolonge la période jusqu'en 1960 et est rattaché aux archives de la direction de Jean Vilar (1951-1963) ${ }^{9}$. Pour cette période, on trouve des documents relatifs à la vie du théâtre et au bâtiment ainsi qu'à la programmation de Chaillot, TNP et hors TNP, classée par spectacle (programmes, photographies notamment). Cependant l'essentiel des archives a été donné par Jean Vilar aux Archives nationales en 1966. Notons en outre pour cette période l'existence de deux fonds primordiaux conservés à la Maison Jean Vilar à Avignon, propriété de l'Association Jean Vilar, mais mis en dépôt à l'antenne BnF sur place. Il s'agit des archives personnelles de Jean Vilar ${ }^{10}$ qui comprennent de nombreux textes et notes manuscrites de Vilar sur la vie du théâtre et sur ses mises en scène, des documents administratifs, des photographies, des programmes et les questionnaires remplis par le public. De même, le fonds Jean Rouvet ${ }^{11}$, administrateur du TNP de 1951 à 1959, réunit une très riche documentation sur le fonctionnement du TNP - administration, budget, personnel, tournées. On pourrait citer aussi les deux fonds Gérard Philipe, celui de l'Association Jean Vilar, consacré à sa carrière théâtrale, et celui de la BnF plus tourné vers sa vie personnelle et celle d'Anne-Marie Philipe, mais contenant par exemple l'enregistrement rare de Nucléa cité plus haut. Enfin, notons que les archives audiovisuelles du TNP pour la période Vilar ont été données plus tard avec celles de son successeur. Elles sont complétées par un ensemble de bandes conservées à la Maison Jean Vilar.

10 À l'inverse de son prédécesseur, Georges Wilson décide en 1972, à la fin de son mandat, de se tourner vers la Bibliothèque nationale. Cette orientation correspond à une dynamique plus globale des années 1970 qui met la BN au cœur du développement et de la préservation du patrimoine théâtral. Sous l'impulsion d'André Veinstein et Cécile Giteau sera bientôt créé le département des Arts du spectacle. Parallèlement, les disciples de Vilar, Armand Delcampe, Paul Puaux et Jean Rouvet, bientôt à la tête de l'Association Jean Vilar, se préoccupent de la sauvegarde de la mémoire de leur patron décédé en 1971 et font appel à la BN pour fonder avec elle la Maison Jean Vilar qui ouvre ses portes en 1979. C'est dans ce contexte que Georges Wilson se tourne vers la BN. Le fonds des archives du TNP sous sa direction ${ }^{12}$ est très complet. Il contient des documents administratifs, des documents de mise en scène, des maquettes de décors, des affiches, des programmes, des photographies et des coupures de presse sur l'ensemble de la période 1963-1972. Il comprend en outre toute la mémoire audiovisuelle du théâtre depuis l'arrivée de Jean Vilar en 1952. Il a été complété en 1976 
par le don de 128 costumes, comme celui d'Edwige Feuillère dans La Folle de Chaillot en 1965 ou de Georges Wilson lui-même dans Le Roi Lear en 1967. Ce fonds entre en résonnance avec le fonds Jacques Le Marquet ${ }^{13}$, chef de la régie construction du TNP de 1954 à 1972, décorateur et costumier, fonds donné à la BnF à partir de 1999 en plusieurs étapes. Jacques Le Marquet signe la plupart des scénographies des spectacles de Georges Wilson. Il est très disert sur le fonctionnement du théâtre à l'époque et apporte un regard de l'intérieur précieux ${ }^{14}$. Comme mentionné dans l'introduction, on trouvera aussi des prolongements documentaires pour cette période dans le fonds Armand Gatti.

11 Les successeurs de Georges Wilson, Jack Lang, directeur du Théâtre national de Chaillot de 1972 à 1974 puis André-Louis Perinetti, directeur de 1975 à 1981, confient à leur tour leurs archives à la $\mathrm{BN}^{15}$, de même qu'Antoine Vitez, directeur de 1981 à $1988^{16}$. Ces fonds contiennent des documents selon la même typologie que le fonds TNP-Wilson. Ils incluent aussi d'importantes archives audiovisuelles. Toutefois la période Vitez n'est pas exhaustive, une partie des documents audiovisuels étaient restés au théâtre et ont été versés récemment aux Archives nationales. Pour les mises en scène d'Antoine Vitez à Chaillot, on notera qu'outre les archives, le département conserve une dizaine de costumes pour les Molière comme celui de Didier Sandre pour Arnolphe ou celui de Nada Strancar pour Elvire, celui de Jean-Yves Dubois pour Hippolyte de Robert Garnier, et d'autres pour Faust ou L'Orfeo. De même, les Arts du spectacle conservent un ensemble important de plus de 170 marionnettes pour les spectacles joués à Chaillot sous la direction d'Antoine Vitez, notamment pour La Tentation de Saint-Antoine d'Alain Recoing ou pour Les Aventures du petit père Lapin de Pierre Blaise. Pour la période Vitez, peut aussi être signalé un ensemble modeste par la taille mais important au regard des liens de collaboration entre Antoine Vitez et la donatrice, Marie Étienne, secrétaire générale de Chaillot au début de son mandat.

Les sources pour l'histoire des théâtres du Trocadéro et de Chaillot au département des Arts du spectacle de la BnF sont donc foisonnantes. Leur intérêt se fonde aussi sur leur complémentarité, complémentarité entre les différents supports - un costume, sa maquette et une photographie du spectacle - et complémentarité entre les différents fonds et séries. Aux résonnances mentionnées plus haut, on peut ajouter d'autres ramifications : les lettres de Jean Vilar à Jean-Louis Barrault entre 1954 et 1959 dans le fonds Renaud-Barrault, les documents sur les tournées du TNP-Villeurbanne à Chaillot et les spectacles de Roger Planchon, ceux sur Les Baracos en 1978 dans le fonds de leur auteur Jean-Jacques Varoujean, etc. L'exposition Chaillot, une mémoire de la danse, présentée à la $\mathrm{BnF}$ de mai à août 2018, a bien montré la diversité et la richesse des collections du département des Arts du spectacle et au-delà celles de la BnF grâce aux photographies d'agences de presse du département des Estampes et de la Photographie, à la presse elle-même conservée au département Droit, économie, politique, sans compter les ressources audiovisuelles publiées au département de l'Audiovisuel, et à l'ensemble des livres et revues, français et étrangers, qui continuent à accroître le nombre de documents accessibles aux chercheurs. 


\section{NOTES}

1. RT-3413.

2. 4-MAQ-8624 et 4-MAQ-8681.

3. COL-148.

4. COL-155.

5. 8-rsupp-590 et 8-rsupp 860.

6. 4-ICO THE- 707 à 714.

7. 4-ICO ARC-23 et FOL-ICO ARC-74.

8. Les archives Firmin Gémier, Paul Abram et Paul Aldebert sont regroupées sous la cote COL-172.

9. COL-71.

10. JV.

11. JR.

12. COL-44, inventaire en salle de lecture.

13. COL-53, inventaire en cours.

14. Voir Anaïs DUPUY-OLIVIER, Jacques Le Marquet, scénographe aux multiples talents (1953-1990). Thèse de l'École nationale des chartes, 2007.

15. COL-300, inventaire en cours.

16. COL-45.

\section{RÉSUMÉS}

Dans la longue histoire du palais du Trocadéro puis du palais de Chaillot, de 1878 à nos jours, les spectacles sous toutes leurs formes ont joué un rôle central. La Bibliothèque nationale de France, et en particulier son département des Arts du spectacle, conserve d'abondantes traces de cette programmation et de ceux qui l'ont faite. Elle est documentée dès l'origine dans la collection fondatrice du département, la collection Auguste Rondel. Les séries de programmes, d'affiches, de photographies, de presse et du dépôt légal du Web prolongent cette documentation jusqu'à aujourd'hui. En outre les Arts du spectacle conservent une partie importante des archives du théâtre de Firmin Gémier à Antoine Vitez, avec notamment des documents audiovisuels. D'autres fonds comme le fonds Jacques Le Marquet et le fonds Jean Vilar déposé à la Maison Jean Vilar à Avignon complètent ce riche panorama de sources à la disposition des chercheurs.

\section{INDEX}

Mots-clés : Théâtre national populaire, Théâtre national de Chaillot, Palais de Chaillot, Palais du Trocadéro, Trocadéro, Gémier (Firmin), Le Marquet (Jacques), Vilar (Jean), Vitez (Antoine), Perenetti (Jean-Louis), Wilson (Georges), Lang (Jack), Rondel (Auguste, Bibliothèque nationale de France 
AUTEUR

JOËL HUTHWOHL

Archiviste paléographe, directeur du département des Arts du spectacle de la Bibliothèque nationale de France 


\title{
De la bobine au fichier numérique, une archéologie des archives audiovisuelles et sonores du Théâtre national de Chaillot
}

\author{
Sandrine Gill
}

Dans le domaine du spectacle vivant, qu'entend-on par archives audiovisuelles? Chaque répétition ou représentation étant par essence unique, liée à une circonstance, un public, un contexte spatial et temporel, l'archivage audiovisuel d'un spectacle, s'il ne concerne pas l'ensemble, sera nécessairement sélectif. Les récents versements aux Archives nationales des enregistrements sonores et visuels du Théâtre national de Chaillot réalisés depuis le début des années 1970 constituent à cet égard un beau cas d'école ${ }^{1}$. Cette démarche d'archivage audiovisuel, commune à d'autres théâtres, permet d'analyser non seulement les objectifs et les choix de l'institution, mais aussi la notion même d'archive. Selon la définition du code du Patrimoine, l'archive est un document d'activité ${ }^{2}$, qui, dans le contexte du théâtre, est réalisé en interne, archivé sur des supports matériels d'enregistrement analogiques ou numériques ou sous forme de fichiers nativement numériques. À la différence des productions professionnelles et à quelques exceptions près, les images ne sont pas montées, retravaillées et, lorsque c'est le cas, le théâtre a aussi conservé la ou les versions brutes issues d'une ou plusieurs caméras. Les représentations ont, dans la plupart des cas, été filmées in extenso, depuis l'installation des spectateurs ou le lever du rideau, au départ des spectateurs et parfois même au démontage des décors. Les archives sonores sont, quant à elles, principalement constituées de bruitages. 
Figure 1

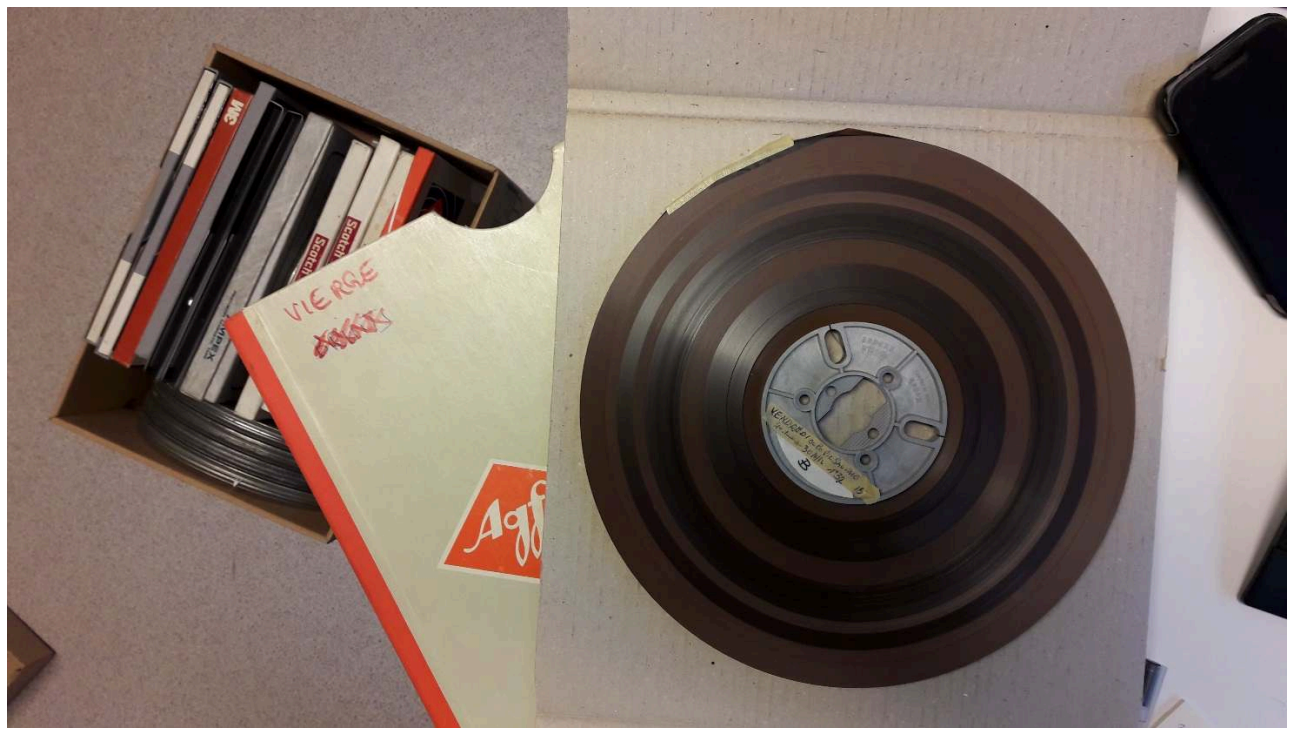

Réception des archives sonores du Théâtre national de Chaillot aux Archives nationales en 2017

Pour les plus anciennes d'entre elles, ces archives datent de l'époque du Théâtre national populaire, sous la direction de Georges Wilson (1963-1973). La pratique se perpétue avec le Théâtre national de Chaillot sous les directions d'André-Louis Perenetti (1974-1981), Antoine Vitez (1981-1988), Jérôme Savary (1988-2000), Ariel Goldenberg (2000-2008), Dominique Hervieu (2008-2011). Une partie des archives de la direction de Didier Deschamps (2011-2014) a déjà été versée. Seule la direction de Jack Lang (1973-1974) n'est pas représentée dans ces versements. Alors même que le filmage du théâtre retient l'attention ${ }^{3}$, il est frappant de constater la permanence de cette politique au fil des années. Cette étendue chronologique est aussi particulièrement significative à l'aune de l'évolution des techniques d'enregistrements audiovisuels et sonores, de l'usage de la pellicule ou de la bande magnétique au fichier nativement numérique. Compte tenu de cette complexité, le traitement archivistique de ces versements a suscité une démarche qui s'apparente aux procédés du monde de l'archéologie: relevés de terrain, inventaires du corpus, études minutieuses des inscriptions sur les supports d'enregistrement, identification et positionnement des strates successives de productions et de copies, repérage des imports, restitution des techniques de conservation dans le temps, autant d'étapes et de procédés méthodologiques de restitution du contexte de production.

\section{Entrer au Théâtre national de Chaillot en poussant la porte des Archives nationales}

\section{Particularité de ces versements parmi les fonds conservés aux Archives nationales}

En 1966, Jean Vilar versait aux Archives nationales les archives papier du Théâtre national populaire, «un symbole, qui recouvre l'esprit d'une époque, son utopie à propos de la culture, qui, en ce temps de grande mouvance, apparaissait comme le meilleur chemin pour trouver sa place dans la société nouvelle, comme le bouclier 
contre toutes les barbaries. ${ }^{4}$." Depuis lors, d'autres versements d'archives administratives sont venus compléter ces premières entrées sur support papier ${ }^{5}$. En revanche, aucun versement d'archives audiovisuelles et électroniques du Théâtre national de Chaillot n'avait été effectué jusqu'à présent. Ils viennent compléter les fonds audiovisuels reçus d'autres administrations et établissements publics ${ }^{6}$.

Les fonds des Archives nationales sont complémentaires des archives du Théâtre national de Chaillot conservées dans d'autres institutions, notamment la Bibliothèque nationale de France ${ }^{7}$, la Maison Jean Vilar, l'Institut national de l'audiovisuel et le Centre national du costume de scène et de la scénographie. Cette dispersion des sources, mais aussi la distinction des filières et des méthodologies d'archivage en fonction des typologies de supports, contribuent à la vision fragmentée de l'activité du théâtre. Les archives audiovisuelles et sonores sont arrivées aux Archives nationales sans documentation associée. Aucun registre ou fichier ne documente la réalisation de ces enregistrements (objectifs, dates, spécificités techniques, auteurs et coauteurs, contrats avec le metteur en scène, les acteurs, etc.) et de leurs copies. Pour un archiviste, cette lacune implique de reconstituer, aussi fidèlement que possible, les conditions de production et de conservation de ces archives qui représentent une masse importante de supports matériels et immatériels (1821 supports audiovisuels et 576 vidéos numériques, 368 supports sonores). Tels quels, ces supports sont quasiment inexploitables. Tout au plus peut-on les rattacher à un spectacle en particulier.

\section{Les conditions de consultation des archives audiovisuelles}

$5 \quad$ Entre l'arrivée de ces versements aux Archives nationales et leur découverte par les premiers « lecteurs », ou plutôt « auditeurs ", le laps de temps a été relativement court. De fait, dès 2017, chercheurs de l'ANR ÉCHO et étudiants en Études théâtrales l'université de la Sorbonne Nouvelle-Paris 3 sont venus écouter et regarder une sélection d'archives audiovisuelles de Chaillot, les exploiter, les interpréter, leur donner un sens ${ }^{8}$. Dans le cadre d'un projet sur le son au théâtre, les étudiants, dirigés par Marie-Madeleine Mervant-Roux et Joël Huthwohl, ont effectué un patient et méticuleux travail d'écoute des vidéos avant de confronter leurs impressions au visuel. Une double initiation au monde des archives et à l'écoute du son au théâtre, une expérience marquante : «j'ai découvert le monde des archives du spectacle vivant que je ne connaissais pas. On est habitué à voir des captations, à appréhender un spectacle dans son ensemble, à analyser la mise en scène en associant le son et l'image, à considérer le jeu de l'acteur à travers son corps et sa voix. Travailler sur les archives sonores du Théâtre de Chaillot a bouleversé ma perception du théâtre et du monde qui m'entoure. ${ }^{9}$ " Pour ces chercheurs et étudiants, le caractère non-professionnel des enregistrements, avec leurs sons "parasites » (réactions du public, effets acoustiques, etc.), a constitué un avantage, laissant entendre des conditions de représentations plus proches de la réalité d'une salle de spectacle.

6 Dans les conditions contraintes d'une salle de lecture provisoire des Archives nationales, le casque sur les oreilles, les étudiants ont entendu une version numérique d'un enregistrement effectué à l'origine sur un autre type de support. Or, à l'ère du numérique, les questionnements de Céline Loriou sur le goût de l'histoire radiophonique pourraient se transposer à l'histoire du théâtre filmé : «la démarche historienne implique de se poser la question des conditions de production de l'archive. 
Pourquoi telle émission est-elle accessible directement sur le poste de consultation, sous forme de fichier numérique, alors que telle autre est consultable sur CD tandis que d'autres encore ne sont pas accessibles car toujours sur bandes magnétiques ou que certaines n'ont même pas été conservées une fois diffusées ? ${ }^{10}$ Prendre en compte les conditions de production des archives audiovisuelles du Théatre national de chaillot nécessite de s'appuyer sur la réalité matérielle ou immatérielle, dans le cas des fichiers numériques, des supports d'enregistrements. D'autres éléments, telles que les dates et circonstances de représentation (en public ou non, répétition, générale, dernière...) et lorsque les informations sont disponibles, les conditions de tournage, permettent de qualifier l'archive, d'en constituer les métadonnées.

\section{Une première phase d'identification et de classement des archives}

7 Les versements du Théâtre national de Chaillot constituent un beau cas d'école, révélateur de la complexité d'interprétation des sources premières. Justine Dilien, archiviste en mission, a d'abord patiemment collecté et identifié les supports et fichiers dans les locaux du théâtre ${ }^{11}$. Sans possibilité de visionnage in situ, l'identification première des sujets filmés ou enregistrés a pu être effectuée sur la seule foi des inscriptions sur les supports ou des titres des fichiers. La connaissance d'informations annexes, telle la campagne de numérisation lancée en 2013 par l'Institut national de l'audiovisuel, a expliqué la présence d'un certain nombre de DVD portant son logo : à l'issue de cette opération, l'Institut a restitué à Chaillot une copie basse définition sur DVD et les supports originaux. Ces archives ont ensuite été classées selon une logique chrono-thématique qui tenait compte des directions, saisons théâtrales et successions de spectacles. Pour cela, Justine Dilien s'est appuyée sur des sources secondaires : listes de programmation, notices des Arts du spectacle de la Bibliothèque nationale de France relatives au Théâtre national de Chaillot et d'autres publications sur le sujet.

\section{La difficulté liée à l'interprétation des supports et des fichiers}

8 Ce classement a fait apparaître une variété de types de supports audiovisuels, tant analogiques que numériques, des bobines de films de $16 \mathrm{~mm}$ et $35 \mathrm{~mm}$ de l'époque du Théâtre national populaire aux DVD pour les périodes les plus récents. À partir de la fin des années 70, la cassette analogique U-Matic, ancêtre de la VHS, semble avoir eu le vent en poupe avant d'être supplantée par la Betacam dans sa version analogique puis numérique. Certains supports numériques, la Dvcam et le DVD, auraient été utilisés pour des fonctions de copies uniquement. Pour les captations sonores et leurs copies, la bande magnétique est le support le plus couramment utilisé, suivi par la cassette audio, le mini-disque, la disquette et, pour les copies, le CD. Quant aux fichiers numériques, présents uniquement dans le versement d'archives audiovisuelles, ils sont de deux natures : captations nativement numériques ou issues d'opérations de numérisation. 
Figure 2

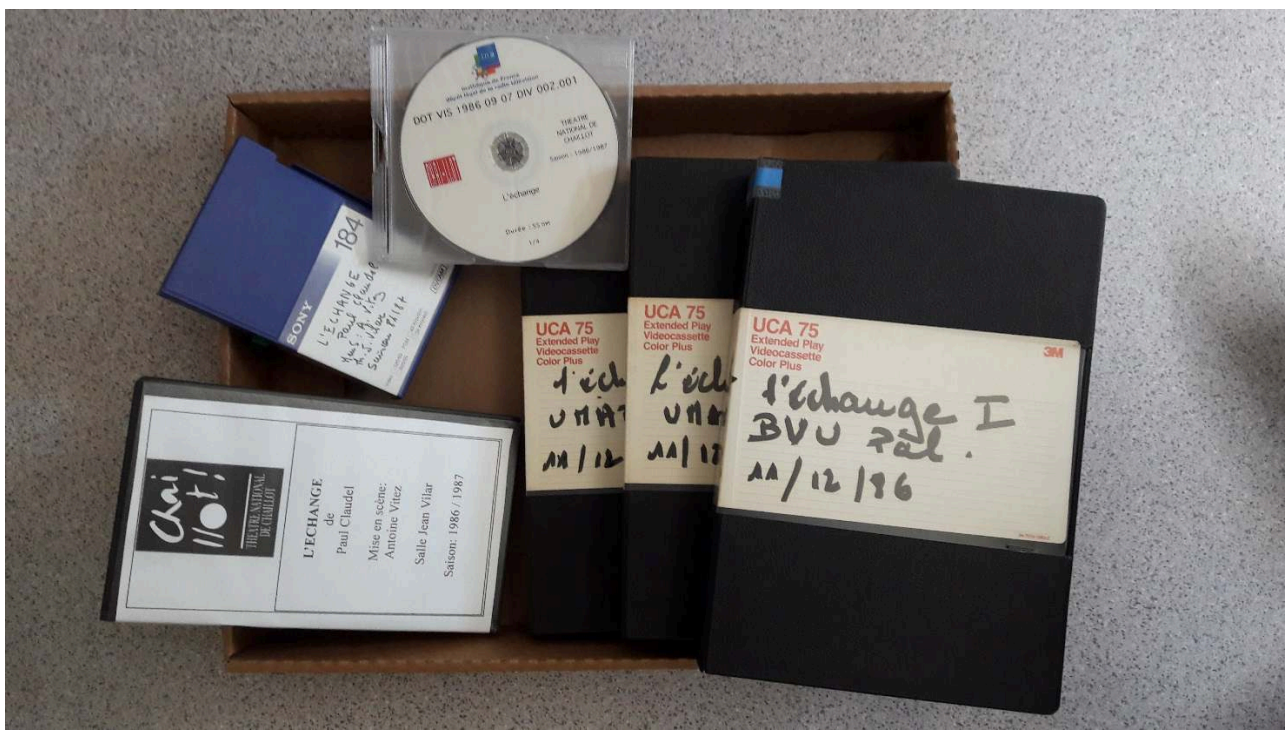

Typologie de supports pour un même spectacle, L'Échange de Paul Claudel, mis en scène par Antoine Vitez en 1986 (Arch. nat. 20160438/58)

9 À ce panorama de supports et fichiers, significatif de l'évolution rapide des techniques audiovisuelles pendant la seconde moitié du XXe siècle, s'ajoute le fait que le théâtre a conservé une multitude de supports pour un même spectacle. Ainsi la pièce Ubu roi d'Alfred Jarry, mise en scène par Antoine Vitez en $1985^{12}$, figure sur quinze U-Matic, une Dvcam, une VHS et deux DVD. Pour Irma la douce, une comédie musicale d'Alexander Breffort mise en scène par Jérôme Savary du 27 avril au 1er juillet 2000 en salle Jean Vilar ${ }^{13}$, la configuration est différente: deux Betacam SP, trois Dvcam, deux VHS, six DVD, quatre fichiers numériques. À quoi ces supports et fichiers correspondent-ils? Pourquoi le théâtre a-t-il conservé l'ensemble? Pour un même spectacle, nous avons un ensemble d'archives audiovisuelles, difficiles à qualifier de prime abord.

\section{L'archiviste, archéologue au chevet des archives audiovisuelles et sonores}

\section{Une méthodologie à la croisée de l'archivistique et de l'archéologie}

Le travail d'identification, de hiérarchisation et de classement des supports s'est poursuivi aux Archives nationales. Ne pouvant s'appuyer sur une expérience similaire de traitement archivistique de versements audiovisuels de théâtre, il est apparu nécessaire de développer une méthodologie spécifique qui, à l'instar d'une fouille archéologique, repartait des sources pour étudier la «culture matérielle " constituée par les supports d'enregistrements. Cette méthodologie peut être déclinée en quatre étapes :

11 1. relever précisément les informations sur les supports,

12 2. les confronter à un visionnage dans la mesure du possible,

13 3. écarter les « intrus » qui ne relèvent pas de la catégorie des archives, 
Figure 3

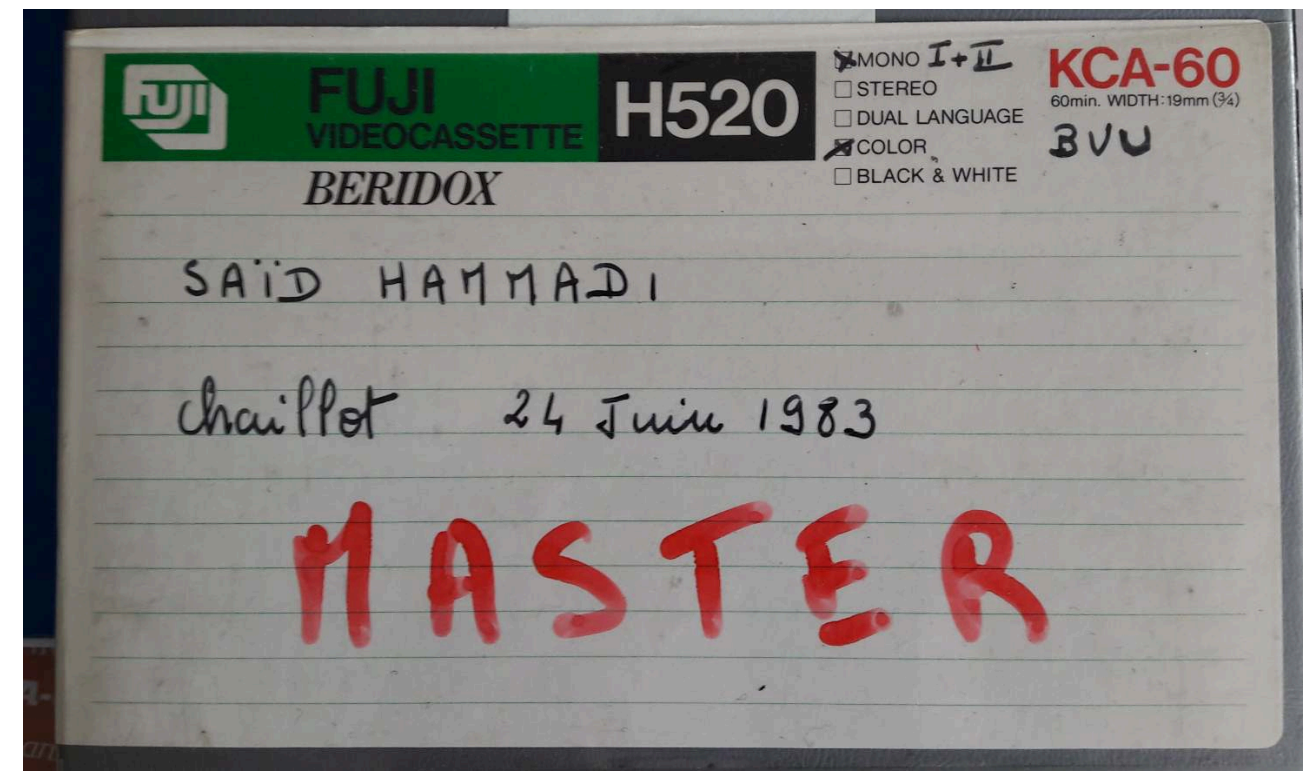

Inscription sur une boite de cassette U-Matic du spectacle Entretien avec Saïd Hammadi, ouvrier algérien de Tahar Ben Jelloun mis en scène par Antoine Vitez en 1982 et 1983 (Arch. nat. 20160438/20)

\section{Relevé de terrain}

Dans un premier temps, le relevé des inscriptions sur les supports est une opération que l'on pourrait comparer à un relevé de terrain archéologique. Il s'agit de noter toutes les informations de nature technique (durée de l'enregistrement ou, à défaut, de la cassette ou du support) et thématique (sujet, type, date, cadre et lieu de la captation). Pour certains spectacles, ces indications s'avèrent très riches, car elles permettent de reconstituer le processus de captation. 
Figure 4

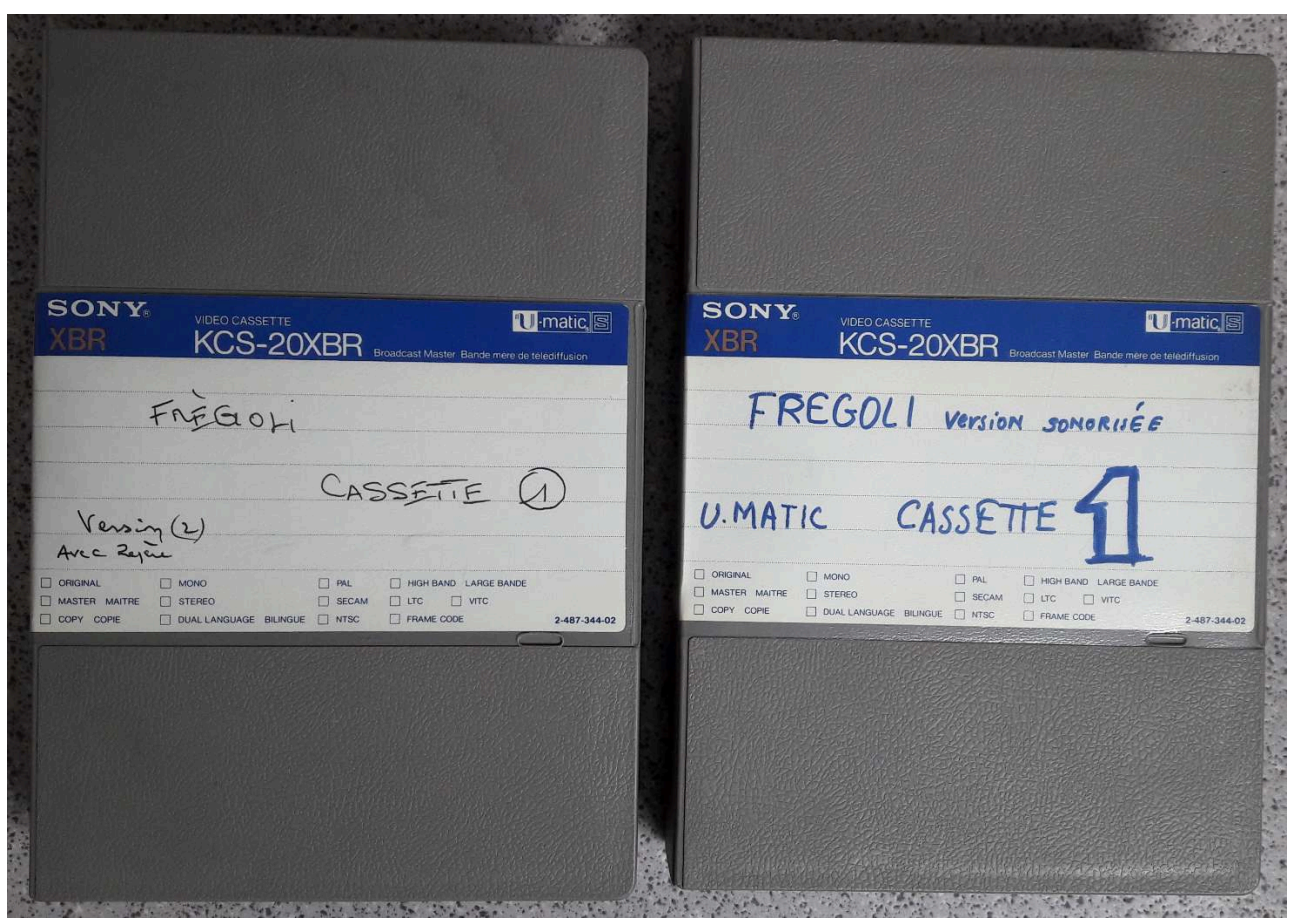

Cassettes U-Matic du spectacle Fregolo de Jérôme Savary, mis en scène en 1991 (Arch. nat. 20160438/91)

Représenté en salle Jean Vilar du 5 avril au 29 juin 1991, le spectacle intitulé Fregoli de Jérôme Savary figure sur quatorze cassettes U-Matic ${ }^{14}$. Le relevé des indications inscrit sur les supports permet de comprendre leur logique et de les classer. Non seulement les répétitions ont été filmées, mais les représentations en public ont fait l'objet de captations distinctes «version 1 », "version 2 », avec ou sans le son. Enfin la version Master a été dupliquée.

\section{Répétitions}

- 1 U-Matic S « Filage », datée du 14 février 1991 : cassette de 20'

- 1 U-Matic S « Filage », non datée : cassette de 20'

Captations du spectacle

- 2 U-Matic « 1re et 2e parties »: cassettes de 20'

- 1 U-Matic «Version 1 » avec une étiquette de la société «Vidéo duplication maintenance »

- 2 U-Matic $S$ « Version 2 avec repère » : cassettes de 20'

- 2 U-Matic S PAL MASTER « son définitif " $n^{\circ} 1$ et $n^{\circ} 2$ : cassette de $20^{\prime}$

- 3 U-Matic $S$ « Version sonorisée 1, 2, 3 » : cassettes de 20'

\section{Copies sur supports analogiques}

- 2 U-Matic PAL MASTER « son définitif » $\mathrm{n}^{\circ} 1$ et $\mathrm{n}^{\circ} 2$ : cassettes de $20^{\prime}$

Par la suite, les captations de ce spectacle ont été sauvegardées sur une cassette Dvcam, support numérique. La durée précise de l'enregistrement figurant sur cette cassette Dvcam n'est pas connue, mais la durée de la cassette, 124 minutes, nous renseigne sur une durée maximale. Enfin la présence de deux DVD INA indique que certains supports d'origine, probablement les deux cassettes U-Matic Master, ont été numérisés. Le théâtre en a extrait deux fichiers numériques de 60 et 43 minutes. 


\section{Visionner certains supports, les comparer avec les données contenues dans les fichiers}

Figure 5

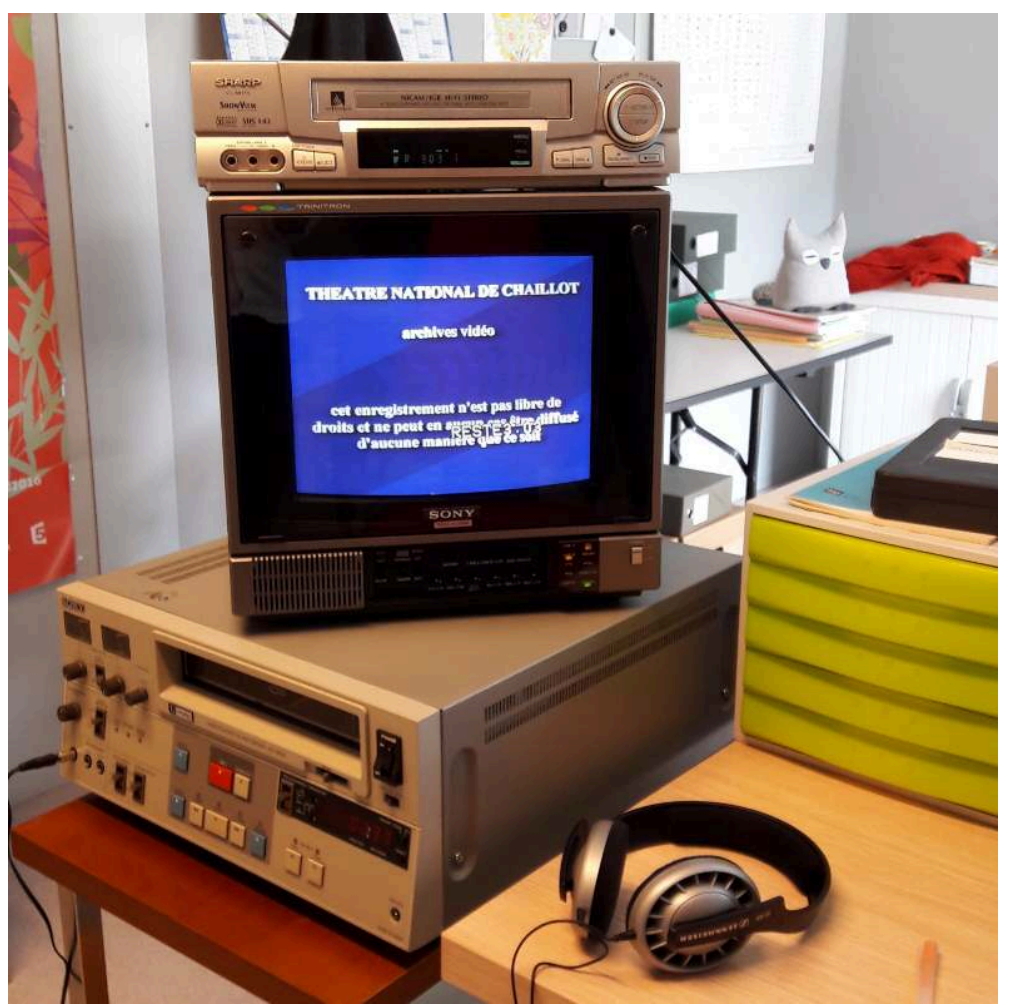

Visionnage de cassettes U-matic et VHS aux Archives nationales, Fontainebleau, 2017

21 Dans certains cas, le visionnage partiel de la captation (début, milieu, fin) s'est avéré essentiel pour identifier un spectacle, corriger une interprétation erronée ou repérer des «intrus ", les copies personnelles sur des supports contenant à l'origine des enregistrements de pièces de théâtre. Ainsi, dans un cas, contrairement à ce qui était indiqué sur l'étiquette d'une cassette, les images étaient celle de l'arrivée du Tour de France. D'autres types de supports ont été éliminés, notamment les copies d'émissions de télévision, conservées à l'Institut national de l'audiovisuel, au titre du dépôt légal. $\mathrm{Au}$ fur et à mesure de ce travail d'identification, l'instrument de recherche était amendé. Cette opération de contrôle par visionnage s'est limitée aux supports les moins endommagés, pour lesquels l'atelier audiovisuel du département de l'Archivage électronique et des archives audiovisuelles des Archives nationales possédait des appareils de lecture : cassettes U-Matic, VHS, Dvcam, DVD. Les bobines de films de 16 ou $35 \mathrm{~mm}$, supports les plus anciens menacés par une dégradation rapide, ont été systématiquement numérisées.

Le visionnage des DVD s'est révélé être particulièrement instructif et a permis de distinguer trois catégories : certains portent la mention manuscrite du TNC et ont été gravés par le théâtre, d'autres ont peu d'indication sinon un titre, enfin un grand nombre d'entre eux sont issus de l'opération de numérisation de l'INA, comme l'indique leur étiquette. La comparaison des DVD INA et des fichiers numériques a mis 
au jour une autre pratique interne : l'extraction des fichiers de numérisation pour une conservation sur un ordinateur ou un disque dur. Les durées d'enregistrement de tous les fichiers numériques versés aux Archives nationales sont identiques aux durées des enregistrements des DVD INA. En tenant compte des supports originaux numérisés par l'INA, on comprend alors que les fichiers numériques sont des copies des copies...

\section{Reconstituer la stratigraphie des enregistrements et des copies}

Afin de restituer l'historique de l'archivage, le relevé d'informations et le visionnage ne suffisent pas en tant que tels. L'histoire des supports audiovisuels, avec leurs dates de production, d'usage et leur finalité (enregistrement, archivage ou copie), s'avère précieuse pour se repérer dans le temps. On sait par exemple que les U-matic furent utilisées de 1978 à 1990 pour l'enregistrement et l'archivage de captations audiovisuelles, tandis que la VHS, en usage de 1980 à 1997, avait pour finalité la copie. En numérique, les DVD servent dès 1995 à sauvegarder des vidéos, tandis que les Dvcam (2000-2005) avaient un double usage: enregistrer et archiver l'audiovisuel. Cette évolution des techniques n'est cependant pas linéaire, les chevauchements de dates entre les cassettes VHS et DVD, par exemple, nous amènent à être prudent dans la reconstitution de l'historique des enregistrements et copies.

Figure 6

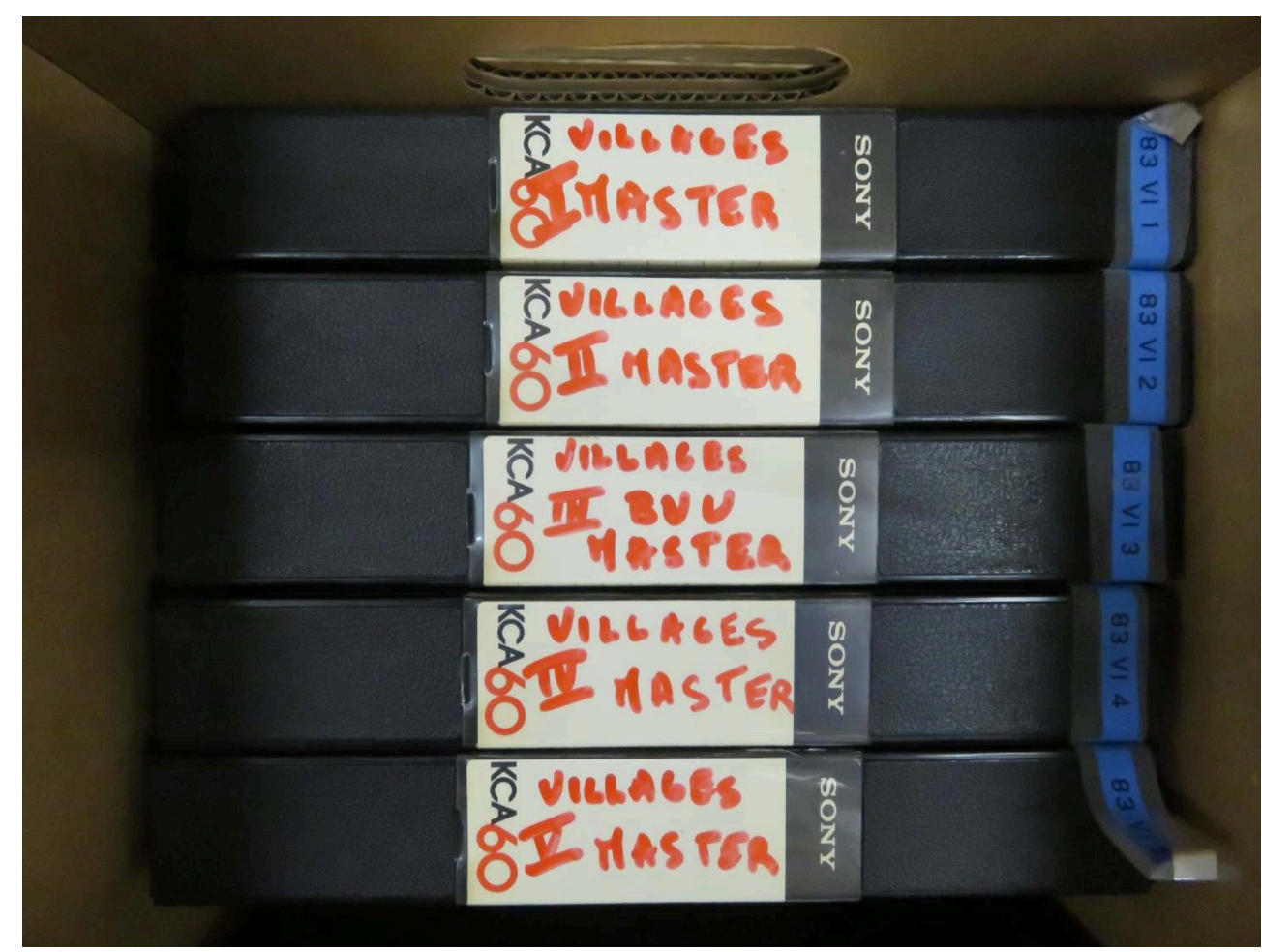

Cassettes U-Matic du spectacle Par les villages de Peter Handke, mis en scène par Claude Régy en 1983 (Arch. nat. 20160438/31) 


\section{Se méfier de l'importance accordée aux originaux}

Si nous avons les originaux, pourquoi garder les copies? Le traitement archivistique des fonds audiovisuels du Théâtre national de Chaillot illustre la fragilité de ce raisonnement. Avec ses nombreux supports analogiques et numériques, complétés par un fichier numérique, l'archivage d'Ubu roi semblait assuré : quinze cassettes U-matic que l'on suppose d'origine, une copie sur cassette VHS, une autre sur une cassette Dvcam, deux DVD produits par l'INA à la suite d'une procédure de numérisation et un fichier numérique. Or l'examen attentif des mentions sur les supports, combiné à des visionnages ponctuels de leur contenu, a conduit à se rendre compte que l'INA n'avait pas numérisé les captations d'origine de la représentation, archivées sur les cassettes U-matic. Ces dernières ayant été endommagées, seuls l'entretien avec Antoine Vitez et une présentation promotionnelle d'Ubu roi avaient été numérisés par l'INA. La cassette VHS, support de copie réputé pour sa qualité médiocre, était quant à elle exploitable et lisible : elle contenait la totalité de la représentation en public d'une durée d'environ deux heures. Support précieux mais fragile dans ce contexte, la VHS a été immédiatement numérisée par les Archives nationales afin de sauvegarder la captation audiovisuelle d'Ubu roi. Éliminer une cassette VHS au prétexte qu'elle ne peut qu'être qu'une copie de mauvaise qualité aurait fait disparaître une archive fondamentale.

\section{Analyser le processus de création d'archives audiovisuelles, alternative à la démarche du metteur en scène}

\section{Conditions de production des archives audiovisuelles}

Une fois le corpus matériel appréhendé dans sa complexité, le sujet même des captations audiovisuelles réalisées par le théâtre peut être analysé. De quoi s'agit-il ? Dans quelles conditions les captations ont-elles été réalisées? Parmi les fonds papier versés pour la première fois aux Archives nationales par Jean Vilar en 1966, la seule référence connue aux enregistrements vidéos concerne le programme mis en place par l'Office culturel pour la communication audiovisuelle (OCCAV) en $1983^{15}$. Un document contenu dans la brochure de l'OCCAV nous informe qu'une captation de la pièce Le père d'August Strindberg, mise en scène par Otomar Krejca et représentée pour la première fois le 18 novembre 1982 en salle Gémier, a été réalisée le 20 décembre. Cette captation ne figure pas dans le sous-fonds audiovisuel du théâtre. Les intentions de ce projet y sont clairement décrites :

«En 1982, avec les captations, l'O.C.C.A.V. innove une nouvelle forme d'utilisation de la vidéo. Trois caméras électroniques sont disposées dans la salle et enregistrent le spectacle en continuité. Une captation est réalisée en une seule journée sans spectateur. Aucune modification n'intervient dans le déroulement de la pièce et le jeu des comédiens. Les captations restituent le spectacle tel qu'il est vu par le public du théâtre.

Afin de conserver en mémoire l'œuvre et son environnement, elle est toujours enregistrée avec la même approche: prises de vue de l'affiche, du spectacle et interview de l'auteur, du metteur en scène ou des acteurs principaux. » 
Réalisées par un professionnel, ces captations de représentations sans public, destinées à constituer une mémoire figée de la création théâtrale, contrastent avec les enregistrements qui composent le fonds audiovisuel conservé aux Archives nationales. Ces derniers proviennent souvent d'une caméra placée au fond de la salle, derrière les sièges les plus hauts. Elle commence à tourner au moment où les spectateurs s'installent, continue pendant l'entracte et s'éteint une fois qu'ils sont sortis, voire une fois les décors démontés ${ }^{16}$. La caméra fixe en fond de scène est le procédé technique le plus couramment employé. Il n'est cependant pas rare de constater le montage de captations réalisées par des caméras disposées à différents emplacements. La captation du Macbeth de Shakespeare, mis en scène par Matthias Langhoff en salle Jean Vilar du 15 février 1990 au 21 mars $1990^{17}$, nous est parvenue sous plusieurs angles de vue, avec des effets de zoom et de plans larges. Le tournage se poursuit parfois hors les salles, comme dans ce film expérimental daté de 1999, contenant un travelling dans un couloir du théâtre associé à un montage d'extraits (chanteuse de jazz, images de guerre, de violences) ${ }^{18}$. Nous n'avons aucune autre information sur le sujet ou le contexte du film.

\section{Des archives d'un théâtre, sous toutes ses formes}

Ces archives témoignent de la vie du théâtre dans des conditions de représentations habituelles. Il n'est pas rare d'avoir plusieurs captations datées pour une même pièce, des répétitions, des enregistrements en public, des reportages en coulisses, mais également des tournées. Pour L'Avare de Molière ${ }^{19}$, mis en scène par Jérôme Savary du 11 mars au 20 juin 1999, le théâtre a conservé une captation d'un filage daté du 27 novembre 1998, mais aussi d'une représentation à Orléans le 21 janvier 1999. Pour sa comédie musicale $\mathrm{Zazou}^{20}$, nous avons aussi bien les captations des représentations en salle Jean Vilar du 24 avril au 30 juin 1990, que de sa tournée au Portugal en 1991.

La vie du théâtre est parfois documentée par des reportages en coulisses, celui du concert d'Annabelle Mouloudji Quartet en mars $1999^{21}$, ou encore de la pièce Le vent dans les saules de Kenneth Grahame mis en scène par Daniel Soulier en salle Jean Vilar du 10 novembre au 30 décembre $1999^{22}$.

Loin de se cantonner aux pièces de théâtre, aux concerts et aux chorégraphies, le fonds audiovisuel révèle des captations d'entretiens, de conférences, des bals organisés à l'issue de représentations chorégraphiques ou des remises de prix. Parmi ces événements, on signalera la captation du bal masqué organisé à la suite de la représentation de Songes de Béatrice Massin le 30 janvier 2010 au Grand Foyer ${ }^{23}$. Plus rares, quelques captations témoignent de l'évolution du lieu. Le reportage filmé sur les travaux de la grande salle du palais du Trocadéro en 1972-1973, avec des vues de démolitions au bulldozer ${ }^{24}$, constitue un précieux complément aux archives papier relatives à l'architecture du Palais de Chaillot. À la différence des spectacles, dont la captation semble avoir été quasi systématique, ces événements annexes ont été filmés et archivés de manière plus sporadique.

Autre catégorie d'archives audiovisuelles, les éléments constitutifs de la mise en scène : des surtitres projetés lors des représentations en langue étrangère pour les archives des années $1960^{25}$, aux incrustations vidéos de Rafael Spregelburd dans les années 2008-2009 dans ses spectacles La Estupidez et La Paranoïa ${ }^{26}$.

31 Enfin des copies de captations reçues d'autres théâtres ont également été conservées par le théâtre, intégrant son fonds d'archives. De l'époque de la direction de Jérôme 
Savary date une copie de la captation Cyrano de Bergerac qu'il avait mis en scène en langue allemande au Bad Hersfelder Festspiele en $1994^{27}$.

\section{Spécificités des archives sonores : écouter autrement le théâtre}

Dans le corpus d'archives sonores, les captations de représentations sont minoritaires : il s'agit essentiellement d'extraits sonores (bruitages, ambiances, compositions musicales) utilisés dans la mise en scène d'un spectacle. Ces archives ne constituent pas une banque de sons réutilisables mais un matériau sonore lié à une pièce spécifique. Deux bandes magnétiques $19 \mathrm{~cm} / \mathrm{s}$ en lien avec L'Atlantide de Pétrika Ionesco, jouée du 26 novembre au 22 décembre 1974, nous sont parvenues ${ }^{28}$. La liste de sons figurant sur la seconde bande magnétique dessine en filigrane une trame sonore de la pièce. Aucune autre archive audiovisuelle de L'Atlantide ne figurant dans les versements du théâtre, cette archive sonore est l'unique témoignage disponible, dans les récents fonds versés, de cette création.

\section{Figure 7}

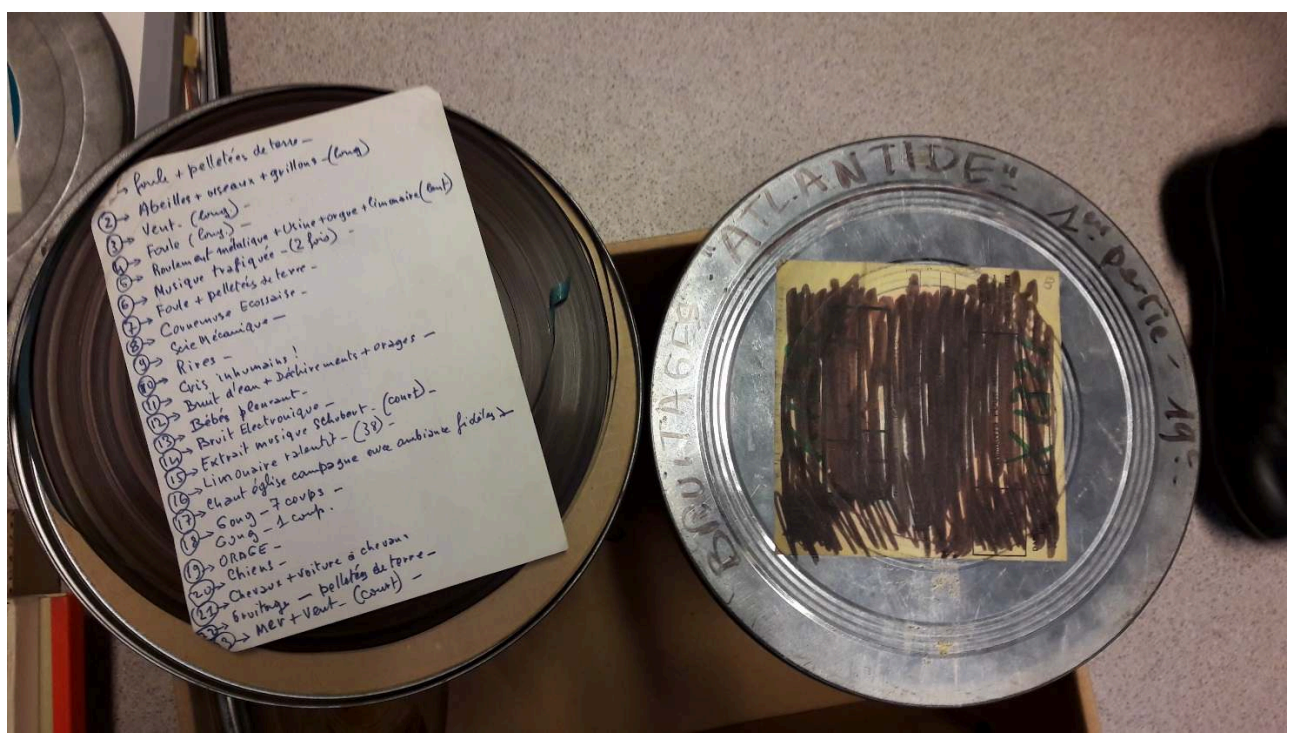

Liste manuscrite des extraits sonores incluse dans la boite de la bobine de la pièce L'Atlantide de Pétrika lonesco mise en scène en 1974 (Arch. nat. 20170371/5)

L'énumération des captations sonores dessine autrement la trame dramatique de cette pièce :

1. Foule + pelletées de terre

2. Abeilles + oiseaux + grillons (long)

3. Vent (long)

4. Foule (long)

5. Roulement métallique + usine + orgue + limouaire (lent)

6. Musique trafiquée (2 fois)

7. Foule + pelletées de terre

8. Cornemuse écossaise

9. Scie mécanique

10. Rires

11. Cris inhumains

12. Bruit d'eau + déchirements + orages

13. Bébés pleurant 


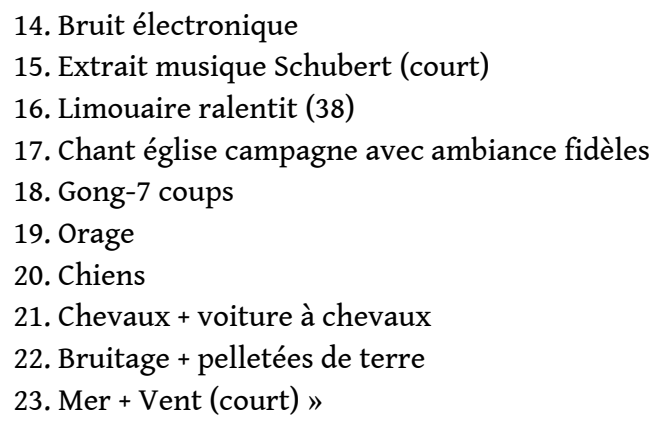

Dans les années 1990, il est toujours courant d'utiliser des bruitages. Pour la représentation d'Andromaque de Jean Racine mise en scène par Marc Zammit ${ }^{29}$, une bande magnétique sonore de $9,5 \mathrm{~cm} / \mathrm{s}$, précisément datée du 22 avril 1992, contient les enregistrements des bruitages suivants :

- bruits de mer

- voix de femmes et pierres

- retour vers la mer

- voix angéliques

- battements sourds »

Figure 8

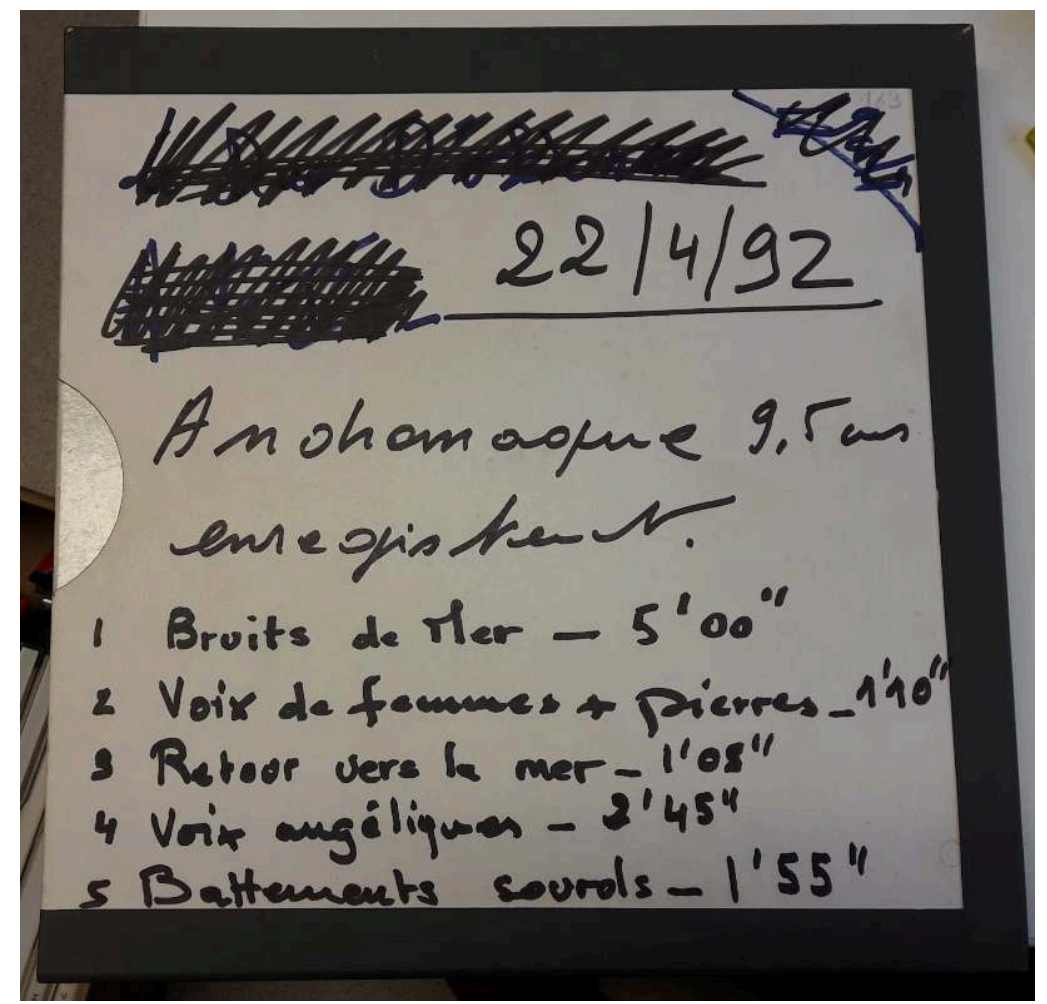

Pochette de la bande magnétique sonore d'Andromaque de Jean Racine mise en scène en 1992 ( Arch. nat., 20170371/55)

De l'Atlantide ou d'Andromaque, seuls les bruitages nous sont parvenus. À l'inverse, l'Entretien avec Saïd Hammadi ouvrier algérien de Tahar Ben Jelloun, mis en scène par Antoine Vitez en 1982 et 1983, n'existe que sous la forme d'archives audiovisuelles ${ }^{30}$. Pour d'autres représentations, des archives complémentaires, audiovisuelles et sonores, ont été conservées. Ainsi Le Soulier de satin de Paul Claudel mis en scène par 
Antoine Vitez en 1987 figure dans le fonds aussi bien sous forme de captation audiovisuelle de la pièce jouée en public $^{31}$, que d'enregistrement sonore d'une répétition du 15 avril ${ }^{32}$. Par ailleurs, la bande magnétique sonore de L'Avare, datée du 15 décembre 1998, laisse présager un travail préparatoire à la mise en scène de Jérôme Savary à partir du 11 mars 1999, conservée également sous forme de captation audiovisuelle ${ }^{33}$.

\section{Poursuivre les campagnes de numérisation pour préserver et communiquer les fonds}

La numérisation des supports physiques demeure à l'heure actuelle la meilleure solution pour préserver les données à long terme et faciliter leur communication. Dans la suite des opérations de l'INA, une première campagne de numérisation des archives audiovisuelles du Théâtre national de Chaillot par les Archives nationales a été lancée en 2017. Elle concernait en priorité les supports les plus fragiles (bobines de films), les captations de représentations publiques qui n'existaient pas dans le fonds sous forme de fichier numérique, certaines captations non identifiées et quelques exemples de captations annexes aux représentations publiques (répétitions). Nombre de pièces de théâtre, numérisées sont ainsi devenues accessibles au public. Parmi les heureuses découvertes, on citera deux bobines de films $35 \mathrm{~mm}$, qui portaient à l'origine la seule mention "Catherine ». Leur numérisation en 2017 a dévoilé la captation de la pièce Catherine, d'après Les Cloches de Bâle de Louis Aragon, mise en scène par Antoine Vitez en 1975 en Avignon dans une production du Théatre des Quartiers d'Ivry ${ }^{34}$. En tant qu'archive, cette captation a pu être reclassée dans l'instrument de recherche dans la catégorie des documents audiovisuels reçus par le théâtre dans le cadre de son activité. Les résultats de cette première campagne ne sont pas exempts de mauvaises surprises : les données contenues sur certains supports trop endommagés n'ont pu être sauvegardées, tandis que d'autres fichiers numérisés, non identifiables au départ, se sont révélés être des captations d'ordre privé, sans rapport avec l'activité du théâtre. La numérisation des archives sonores, prochaine étape, permettra d'affiner leur identification, leur classement et leur mise en consultation.

Pour l'archiviste, les leçons tirées par le traitement des archives audiovisuelles et sonores sont multiples. L'importance des sources premières, matérialisées dans ces versements par une pléthore de supports et des fichiers numériques, est significative d'un aspect peu documenté jusqu'à présent de l'activité du producteur : la volonté de conserver mais aussi de pérenniser, par la pratique des copies sur de nouveaux supports, cette mémoire visuelle et sonore. Dans le processus d'archivage, le relevé des métadonnées descriptives et techniques, aussi incomplètes puissent-elles être dans certains cas, est essentiel à la contextualisation des archives. Sans métadonnées, une cassette U-matic isolée dans un versement tel que celui du Théâtre national de Chaillot ne pourrait être interprétée par un chercheur. Le rôle de l'archiviste, tel un passeur de sens, est d'établir un lien entre la réalité matérielle de la collecte, dans les armoires du théâtre, et la phase de mise en consultation de ces archives en salle de lecture. C'est désormais aux chercheurs, professionnels et amateurs du théâtre de Chaillot, de poursuivre l'interprétation et la valorisation d'un lieu légendaire du monde du spectacle vivant ${ }^{35}$, en croisant les sources disponibles aux Archives nationales et dans d'autres institutions. Les chercheurs de l'ANR ÉCHO et les étudiants en Études 
Théâtrales ont été les premiers à travailler ces archives audiovisuelles. D'autres pistes de recherche sur les films et les sons du théâtre de Chaillot attendent d'être exploitées.

\section{NOTES}

1. En 2016, les Archives nationales recevaient les archives audiovisuelles du Théâtre national de Chaillot (1966-2014) et en 2017 les archives sonores (1967-2011). Les instruments de recherche de ces versements sont en ligne sur leur site Internet: https://www.siv.archivesnationales.culture.gouv.fr/siv/IR/FRAN_IR_055964 (Archives audiovisuelles); https:// www.siv.archives-nationales.culture.gouv.fr/siv/IR/FRAN_IR_056791 (Archives sonores).

2. «Les archives sont l'ensemble des documents, y compris les données, quels que soient leur date, leur lieu de conservation, leur forme et leur support, produits ou reçus par toute personne physique ou morale et par tout service ou organisme public ou privé dans l'exercice de leur activité » (Code du Patrimoine, article L211-1).

3. Dans son article, «Complémentarité des modes d'archivage modernes du spectacle théâtral: archives sonores/archives papier/archives audiovisuelles", Marie-Madeleine Mervant-Roux évoque cette problématique du filmage du théâtre qui a fait l'objet de plusieurs publications, notamment sous la direction de Denis Bablet, Filmer le théâtre, Cahiers Théâtre Louvain, $\mathrm{n}^{\circ} 46$, Louvain-la-Neuve, 1981, et de Béatrice Picon-Vallin, Le film de théâtre, CNRS éditions, coll. Arts du spectacle, Paris, 1997.

4. GODARD (Colette), Chaillot histoire d'un théâtre populaire, Paris, Éditions du Seuil, 2000, p. 21.

5. Voir les articles de Geneviève Profit et de Justine Dilien.

6. Les archives audiovisuelles conservées aux Archives nationales. Panorama sur 35 ans de collecte d'archives audiovisuelles aux Archives nationales, par Martine Sin Blima-Barru, conservatrice du patrimoine, avec la collaboration d'Isabelle Pérez-Bastié, chargée d'études documentaires; intégration des anciens instruments de recherche établis par Agnès Callu et Rosine Lheureux, conservatrices $d u$ patrimoine, instrument de recherche mis à jour en 2016: https:// www.siv.archives-nationales.culture.gouv.fr/siv/IR/FRAN_IR_052868. Ce document distingue trois grandes catégories d'archives audiovisuelles et sonores : les archives orales constituées par les Archives nationales, les archives audiovisuelles produites et collectées par les administrations publiques de l'État, les fonds privés conservés par l'institution.

7. Voir l'article de Joël Huthwohl.

8. Cette initiation à l'étude du son au théâtre s'inscrit dans le séminaire «L'initiation au travail sur archives dans la formation des étudiants en Master d'Études théâtrales: le séminaire «Mémoire des arts du spectacle vivant » de l'université de Sorbonne Nouvelle-Paris 3 dirigé par Aurélie Mouton-Rezzouk, maître de conférences, et Catherine Treilhou-Balaudé, professeure à l'Institut d'études théâtrales, IRET-université de Sorbonne Nouvelle-Paris 3.

9. Citation d'Océane Djabellah-Peillon, étudiante en Master 1 d'Études théâtrales, université de Sorbonne Nouvelle-Paris 3, extraite de l'article de Sandrine Gill et Marie-Madeleine MervantRoux, "Chaillot lieu de tous les arts ", dans Mémoire d'avenir, le journal des Archives nationales, $\mathrm{n}^{\circ}$ 32, octobre-décembre 2018, p 10.

10. LORIOU (Céline) «Faire de l'histoire un casque sur les oreilles: le goût de l'histoire radiophonique », 27 mars 2018, www.gout-numerique.net. Cette publication numérique fait écho 
à l'ouvrage d'Arlette Farge, Le Goût de l'archive, Paris, Seuil, coll. « Points Histoire », 1997 [1 ${ }^{\text {re }}$ éd. : 1989], consacré aux archives papier,

11. Voir l'article de Justine Dilien, op. cit.

12. Arch. nat., 20160438/43.

13. Arch. nat., 20160438/143.

14. Arch. nat., 20160438/91.

15. Arch. nat., 19900195/109.

16. Dans la captation de Britannicus de Jean Racine, mis en scène par Antoine Vitez au Grand Théâtre à partir de novembre 1981, on assiste à l'entracte (Arch. nat., 20160438/21).

17. Arch. nat., 20160438/78.

18. Arch. nat., 20160438/417.

19. Arch. nat., 20160438/130.

20. Arch. nat., 20160438/79.

21. Arch. nat., 20160438/131.

22. Arch. nat., 20160438/134.

23. Arch. nat., 20160438/296.

24. Arch. nat., 20160438/370.

25. Arch. nat., 20160438/371 : surtitres en français de l'opéra en 3 actes de Kurt Weill la Grandeur et décadence de la ville de Mahagonny, mis en scène par Georges Wilson, sur un livret de Bertolt Brecht en novembre 1966.

26. Arch. nat., 20160438/237 : La Estupidez de Rafael Spregelburd, mise en scène du 13 mars au 5 avril 2008 par Marcia Di Fonzo Bo et Élise Vigier en salle Gémier. 20160438/283 : La Paranoïa de Rafael Spregelburd, mise en scène du $1^{\text {er }}$ octobre au 24 octobre 2009 par Marcia Di Fonzo Bo et Élise Vigier en salle Gémier.

27. Arch. nat., 20160438/120.

28. Arch. nat., 20170371/5.

29. Arch. nat., 20170371/55.

30. Arch. nat., 20160438/20.

31. Arch. nat., 20160438/61.

32. Arch. nat., 20170371/37.

33. Arch. nat., 20160438/130.

34. Arch. nat., 20160438/375.

35. «La légende de Chaillot » est le titre de l'introduction de Didier Deschamps dans L'Esplanade, la revue de Chaillot, 2018.

\section{RÉSUMÉS}

En 2016 et 2017, le théâtre national de Chaillot versait aux Archives nationales plus de 1800 supports audiovisuels et 375 supports sonores, analogiques et numériques, ainsi qu'une série de fichiers numériques, représentant environ 450 spectacles. Un fonds imposant, fruit de quatre décennies de captations et d'archivage de pièces, concerts, chorégraphies et d'événements annexes, ainsi que de copies de captations reçues d'autres institutions, une mémoire de l'activité de Chaillot constituée par le théâtre lui-même. Pour l'archiviste, départager les originaux des copies, l'analogique du numérique, les répétitions des tournées, les incrustations multimédia des 
bruitages, relève d'un véritable travail d'archéologue des temps modernes. Au-delà du témoignage de l'adaptation constante du théâtre aux évolutions techniques, ce large corpus de supports interroge la pertinence même de la conservation : une copie de mauvaise qualité pourra se révéler être l'unique survivante d'un original perdu ou détérioré. Par leur complexité et leur richesse, ces archives audiovisuelles et sonores constituent un ensemble unique, inédit aux Archives nationales.

\section{INDEX}

Mots-clés : Théâtre national populaire, Théâtre national de Chaillot, Vilar (Jean), Vitez (Antoine), analogique, numérique, bobine, bande magnétique, VHS, Betacam, U-matic, Dvcam, cassette audio

\section{AUTEUR}

\section{SANDRINE GILL}

Cheffe de projet archivage audiovisuel, département des Archives électroniques et des Archives audiovisuelles [DAEAA], Archives nationales 


\title{
Complémentarité des modes d'archivage modernes du spectacle théâtral : archives sonores/archives papier/archives audiovisuelles
}

\author{
Marie-Madeleine Mervant-Roux
}

En Études théâtrales, le recours à des types d'archives très variés pour l'analyse d'un même objet est une réalité ancienne et familière. Il s'agira ici de formes de complémentarité engageant une catégorie de documents encore quasi inconnue des chercheurs et des étudiants de cette discipline: les enregistrements sonores. Depuis quelques années, ceux-ci sont sortis du long oubli dans lequel ils avaient été tenus, sans avoir pris la place qui pourrait être la leur parmi les matériaux de la recherche. L'une des raisons pour lesquelles on ne les exploite pas davantage, en dehors du désintérêt toujours vérifié pour la dimension auditive générale du théâtre, est qu'ils sont d'un usage difficile. Exigeant du temps et de l'attention (on ne peut pas «feuilleter » ou «visualiser» rapidement un document sonore), fatigants, souvent peu agréables à écouter (les captations de représentations en public, par exemple, comportent de nombreux bruits qui peuvent d'abord être perçus comme parasites par rapport aux sons du spectacle lui-même), ils s'avèrent en outre peu commodes à prendre en note et à décrire. Enfin leur exploitation dans une perspective scientifique pose des problèmes spécifiques ${ }^{1}$. Mais une fois ces obstacles affrontés, leur apport à la recherche s'avère lui aussi spécifique. Je m'arrêterai rapidement sur deux de leurs particularités, car elles fondent en partie les deux modes de complémentarité que je vais successivement évoquer. Première particularité: le fait que le sonore, même enregistré, "vit au présent ", selon l'expression de Daniel Deshays ${ }^{2}$, et dans un espace mal défini, fait oublier facilement que ce qu'on entend en écoutant un document a été " re-produit ", fixé à un moment donné, dans un lieu donné, dans certaines conditions, à l'aide d'un certain dispositif technique et dans une certaine perspective. La trace audible de l'événement a l'évanescence et le charme d'un spectral que l'on se contente souvent de situer d'une façon assez vague. Il convient donc de localiser le plus exactement possible 
le document dans l'histoire (Où l'enregistrement a-t-il été effectué ? Quand ? Par qui ? Comment? Pourquoi ?) et de reconstituer au besoin sa propre histoire, de la prise de son initiale au phonogramme final (copie, montage, nettoyage, etc.). D'où l'importance particulière de tout ce qui peut aider à matérialiser et contextualiser l'archive audio, tout ce qui peut aider à définir les conditions et le dispositif de sa réalisation: programme du spectacle, calendrier des représentations (pour aider à identifier la date de l'enregistrement), archives de gestion (pour l'identification de comédiens dont on ne reconnaît pas forcément la voix, surtout si beaucoup de temps a passé ou lorsque la distribution a connu des modifications), plans de salle, schémas de sonorisation (pour comprendre les phénomènes acoustiques), traces diverses d'un éventuel travail en studio, photographies prises à différentes étapes de la genèse... La seconde particularité des archives sonores est que leur première consultation - leur première écoute - ne pourra plus jamais être reproduite à l'identique. Or, l'omniprésence sociale des images entraîne à penser comme lacunaire tout document sonore, qu'il faudrait impérativement compléter - voire remplacer - par des traces visuelles ou, depuis cinquante ans, audiovisuelles. Dans notre domaine, le théâtre, l'accoutumance à découvrir les spectacles du passé à travers des images (œuvres picturales et photographies dans les ouvrages, captations vidéo dans les cours et conférences, généralisation du Powerpoint dans les colloques) fait éprouver l'absence de celles-ci comme un manque - alors qu'on se lamente rarement sur l'insupportable absence du son lorsqu'on exploite une documentation photographique. Ce qui pousse à privilégier les captations visuelles ou, dans les rares cas où l'on écoute des archives audio de spectacles, à la consultation en amont d'un appareil documentaire. L'expérience acquise en une décennie dans le cadre de deux projets successifs ${ }^{3}$ nous a fait comprendre que pour éviter d'entendre dans un document sonore ce que les informations ou les images connues en amont nous entraîneraient à y trouver (ou à ne pas y trouver), pour éviter de réagir par rapport à ce que nous saurions déjà, ou croirions savoir déjà (du phonogramme, du spectacle enregistré, des artistes, du metteur en scène, etc.), empêchant le son de nous révéler, justement, d'autres réalités encore insoupçonnées, l'idéal est de commencer par l'écoute en aveugle, sans information préalable, l'étude des documents complémentaires ne devant intervenir que dans un second temps. La raison de cette règle, c'est qu'il ne pourra plus y avoir ensuite d' « écoute réduite ${ }^{4}$ » ou du moins " non dirigée » de l'enregistrement sonore. La recherche y perdrait beaucoup. L'écoute non informée, en effet, peut ouvrir des pistes imprévues - et si celles-ci s'avéraient fausses, ce ne serait qu'un moindre mal. Dans la pratique, il est souvent trop tard pour ne rien savoir avant d'écouter une archive sonore, mais le recours aux autres types de documents doit être autant que possible suspendu. La collaboration d'ECHO avec les Archives nationales, traduite dans l'organisation des journées d'étude de juin 2018, m'a permis d'avancer dans l'exploration de chacune des deux formes de complémentarité : documenter l'archive sonore du spectacle pour mieux l'écouter, faire passer l'archive sonore avant les autres types d'archives pour documenter le spectacle). Pour illustrer le premier processus, j'ai choisi de montrer comment le fonds du Théâtre national populaire déposé aux Archives nationales par Jean Vilar en $1966^{5}$ m'a permis de comprendre le processus d'élaboration d'un des titres les plus célèbres de la discographie du TNP : le Lorenzaccio enregistré lors de la «nuit Renault» du 27 novembre 1954. Pour illustrer la façon dont archives sonores et archives audiovisuelles peuvent se compléter dans la tentative - vouée à l'échec, mais nécessaire - de comprendre ce qu'aura été un spectacle du passé, j'ai 
choisi de consulter les enregistrements du spectacle Par les villages créé par Claude Régy au Théâtre national de Chaillot en $1980^{6}$, en commençant par l'enregistrement audio conservé à la $\mathrm{BnF}$, puis en visionnant la captation vidéo récemment déposée aux Archives nationales. L'occasion pour moi de revenir à un metteur en scène dont j'ai étudié l'œuvre avec une équipe de chercheurs il y a quelques années? ${ }^{7}$. La position de Claude Régy à l'égard de l'archivage a longtemps été radicale : «Filmage interdit » ${ }^{8}$. Il nous avait par contre autorisés à réaliser des enregistrements audio de ses spectacles. Ce travail m'a permis de préciser le constat selon lequel une archive audio n'est pas une archive audiovisuelle moins le visuel, mais relève d'une autre catégorie de trace, possédant ses propres fonctions et qu'il existe entre elles, pour le chercheur, une complémentarité potentielle originale.

\section{Complémentarité des archives audio et des archives papier : reconstituer la genèse du Lorenzaccio discographique de 1956}

2 Il existe dans l'histoire des disques de théâtre quelques éditions célèbres. L'une d'elles est le coffret de trois microsillons 33 tours mono contenant, selon les indications figurant sur la couverture, le Lorenzaccio mis en scène et interprété par Gérard Philipe, avec la troupe du TNP, dans la grande salle de Chaillot, le 27 novembre 1954, durant une soirée réservée au personnel des usines Renault. Produit en 1956 [fig. 1], le coffret a connu trois rééditions dans de nouveaux formats : cassettes audio (en 1974), CD (en 2007), livre audio numérique (en 2010). Dès 1956, cet enregistrement a acquis l'aura d'un événement mythologique, dont on peut énumérer les grandes composantes: l'acteur-prince d'une génération dans le rôle-titre, une pièce réputée injouable, très longue, romantique, sentant le soufre, une troupe exceptionnelle composée (dans la distribution de 1954) de Jean Vilar, Daniel Ivernel, Daniel Sorano, Monique Chaumette, Christiane Minazzoli, Mona-Dol, Georges Wilson, Philippe Noiret, Jean-Pierre Darras, Jean Deschamps, et enfin, dans la salle dont la jauge était de 2800 places environ, un public "populaire», les usines Renault constituant alors un bastion ouvrier et syndicaliste. 


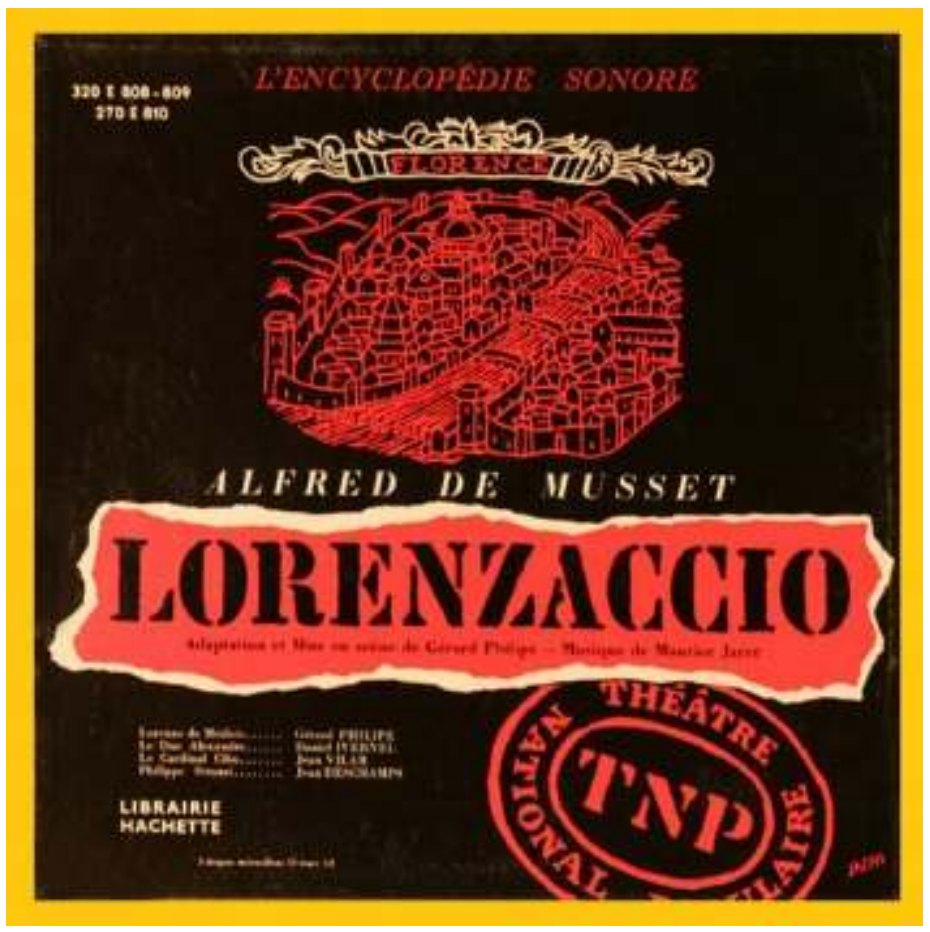

Lorenzaccio, mise en scène de Gérard Philipe (TNP, 1954), couverture du coffret de trois disques 33 tours, L'Encyclopédie sonore-Hachette, 1956 (Référence 320 E 808-809, 270 E 810), conception graphique de Marcel Jacno. Doc. Jacques Hiver. Avec l'accord gracieux de Corinne Juresco et l'aide de l'association des Amis de Marcel Jacno.

3 À l'écoute, ce disque révèle quelques étrangetés, relevées en 2014 par Noémie Fargier, assistante à la recherche dans le projet $\mathrm{ECHO}$, et consignées dans la fiche descriptive qu'elle avait rédigée sur le document: on peut repérer des irrégularités importantes dans l'intensité des sons, en particulier des voix, et dans le rapport entre les voix et les autres bruits de la scène et de la salle. Par exemple dans la scène 3 de l'acte III. Dans le coffret de 1956, celle-ci est séparée en deux éléments : la première partie de la scène se trouve à la fin de la face $\mathrm{A}$ du disque 2, la seconde partie se trouve au début de la face $\mathrm{B}$. [fig. 2 et 2 bis]'. Étant donné la pause non négligeable nécessaire pour le changement de face du microsillon et le redémarrage de l'électrophone, un tel découpage atténuait (et pouvait même supprimer) la perception d'une discontinuité sonore - qui n'échappe pas à une oreille attentive. Il existe une différence dans la façon dont les voix résonnent dans l'espace acoustique alors qu'il n'y a pas de solution de continuité dramatique ou scénique : c'est la même scène qui continue. Juste après l'interruption, il est vrai, un événement se produit : «Lorenzo entre » et un long dialogue commence entre celui-ci (Gérard Philipe) et Philippe Strozzi (Jean Deschamps), mais ce dernier ne bouge pas de la place où il est assis et rien ne justifie la transformation globale du son. L'autre bizarrerie est la présence de "gros plans » vocaux. "À certains moments, avait noté Noémie Fargier, les comédiens paraissent proches, et l'enregistrement de leur voix est saturé ». On pense d'abord aux micros installés sur la scène de Chaillot, dont les comédiens se rapprocheraient à certains moments. Contrairement à ce qu'on a cru longtemps, en effet, la présence de micros est attestée dès l'ouverture du TNP en 1952. On en a plusieurs témoignages, entre autres dans un document permettant d'entendre les répétitions de L'Avare enregistrées en 1952 sur le grand plateau par Michel Polac ${ }^{10}$, ou dans de nombreuses réponses aux questionnaires proposés par les responsables du 
TNP et remplis par les spectateurs après les représentations ${ }^{11}$. Citons ces deux remarques :

«Au début un peu surpris par les différences d'acoustique lors du déplacement des acteurs devant les micros, mais on s'y habitue vite. » (Orchestre A, questionnaires de novembre-décembre 1953).

« Il conviendrait de signaler un défaut de sonorisation de la salle. En effet, la voix de Jean Vilar, cependant belle, grave, au timbre étendu, me parvenait très mal. Les amplificateurs ne devaient, sans doute, pas être bien réglés, car c'est la première fois que cela se produit pour moi. » (Spectateur installé " au-delà de la série A ", même source).

Figure 2

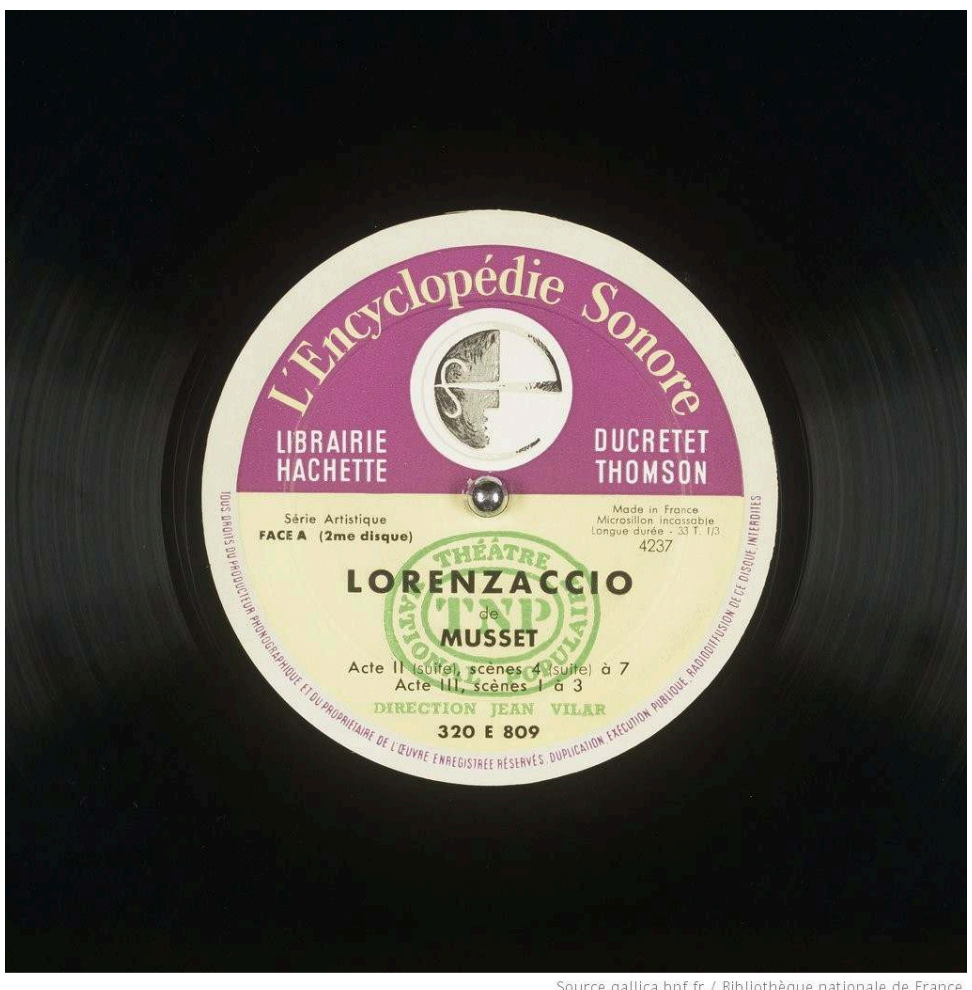

Lorenzaccio, L'Encyclopédie sonore-Hachette, 1956. Face A du disque 2 (BnF, Gallica)

https://gallica.bnf.fr/ark:/12148/bpt6k88081256/f3.media 
Figure 2 bis

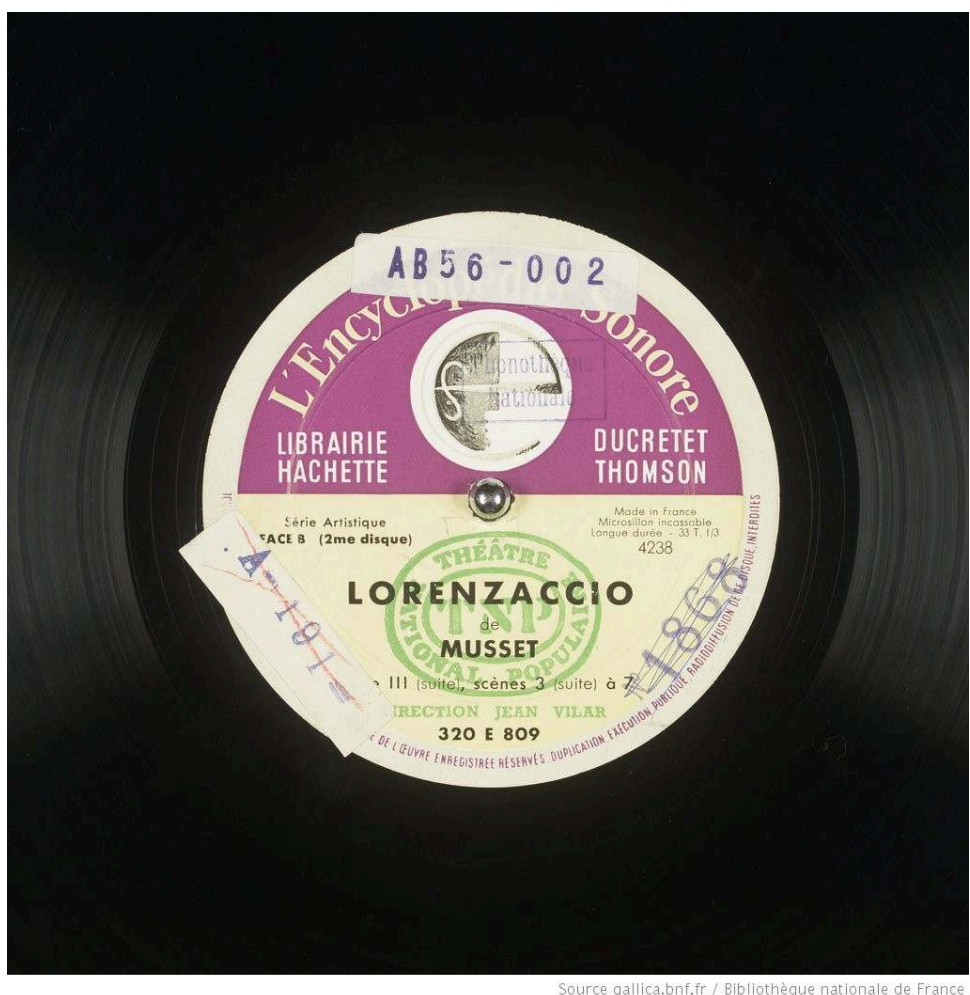

Lorenzaccio, L'Encyclopédie sonore-Hachette, 1956. Face B du disque 2 (BnF, Gallica)

https://gallica.bnf.fr/ark:/12148/bpt6k88081256/f3.media

4 La sonorisation, responsable de certaines gênes, ne peut expliquer les variations brusques d'intensité de la voix en dehors de tout mouvement sensible des acteurs. La raison doit être cherchée ailleurs.

5 C'est là qu'interviennent les documents papier (administratifs, financiers, techniques) du TNP conservés aux Archives Nationales.

6 Ces documents nous apprennent d'abord que l'enregistrement du 27 novembre 1954 n'était ni le seul ni le premier enregistrement du spectacle.

7 Une série de trois enregistrements de représentations avait été organisée par le TNP à Chaillot avant décembre 1953, avec la firme Ducretet-Thomson, dans le but d'éditer un disque. C'est Jean-Wilfrid Garrett, ingénieur son à la radio, qui s'est occupé du montage et de la copie aux studios Geneix. Nous disposons d'une lettre de Jean-Wilfrid Garrett à Jean Rouvet, l'administrateur du TNP, datée du 7 décembre 1953, accompagnée par le devis des "enregistrements et montages du "Cid" et de "Lorenzaccio"». Pour Lorenzaccio : " 1 séance d'étude et installation du matériel et 3 séances d'enregistrement des représentations publiques, soit $20000 \mathrm{Fr}$ [note déjà réglée du 28 mai 53], 4 séances de montage, [sur machine stéréo TNP] soit $20000 \mathrm{Fr}$, une séance de copie [en $38 \mathrm{~cm}$ monopiste], soit $500 \mathrm{Fr}$ ». À ces frais s'ajoutent les sommes dues aux studios Geneix. "Une séance de copie à 14000,4 bandes vierges, 18000 ». Une somme de 40500 ("enregistrements primaires de Lorenzaccio») a déjà été versée, les bandes magnétiques (9) utilisées pour enregistrer le spectacle ont aussi été réglées. 
Une lettre datée du 29 mai 1954 est envoyée au TNP par Jean Chesman, de la part de M. Valoussière, directeur de Ducretet-Thomson, incluant un "ajout au contrat " (contrat non retrouvé à ce jour) :

«Les bandes sont achetées dans l'état. Cependant Gérard Philipe, Monique Chaumette, Jean Deschamps, Georges Wilson ont l'amabilité de se plier au travail complémentaire nécessaire pour parfaire la qualité des bandes (LeCid et Lorenzaccio). Devant le résultat plus que satisfaisant obtenu par la prise directe (en représentation) de Richard II et Don Juan, nous demandons à réenregistrer Le Cid et Lorenzaccio, lors de la reprise des représentations à Chaillot en novembre prochain. Deux prises sont nécessaires. »

Cette lettre nous apprend que certains passages du Lorenzaccio, concernant quatre comédiens importants, doivent être réenregistrés, et cela en public, pour ne pas créer un trop grand décalage acoustique avec les trois prises réalisées dans la grande salle en 1953 pendant des représentations (des prises effectuées en studio ou dans la salle vide de spectateurs étaient exclues). On comprend aussi que les conditions d'enregistrement ont été améliorées et que le travail devrait se faire le plus vite possible, c'est-à-dire en novembre (1954). Le 24 novembre, Jean Deschamps (comédien, interprète de Philippe Strozzi dans le spectacle, par ailleurs très engagé dans "L'Encyclopédie sonore", collection créée par Hachette-Ducretet-Thomson), écrit à Jean Rouvet sur papier à entête du TNP « Mes camarades [...] sont d'accord pour l'enregistrement de Lorenzaccio ce soir jeudi [sic] (première prise) et celui du Cid samedi (2 prises) aux conditions prévues l'an dernier lors des deux enregistrements défectueux. Le syndicat des musiciens est en outre prévenu et un accord est intervenu pour leur paiement. » Une note de Rouvet à Vilar, datée du même jour, éclaire et précise ce courrier: les « camarades » sont les comédiens, les enregistrements sont destinés à "L’Encyclopédie sonore ». Vilar donne son accord. On ne sait pas si la deuxième prise de novembre 1954 a bien eu lieu, mais il est probable que oui et qu'elle a été effectuée durant la nuit Renault, le samedi 27. Mais ce n'est pas tout. Un autre enregistrement du spectacle avait été réalisé par la radio le 19 mars 1953 pour l'émission " Théâtre et Université ». Il a été diffusé le 25 mars (d'après le document de l'INA), précédé d'un débat avec Jean Vilar et Gérard Philipe, débat qui avait en réalité eu lieu après le spectacle. Ce qui est intéressant, c'est que cet enregistrement ne devait être diffusé qu'une fois, selon la demande fermement exprimée par Vilar à M. Gilson, directeur des services artistiques de la RDF, dans une lettre datée du 7 mars 1953. Dans le même courrier, Vilar demandait qu'un enregistrement du spectacle encore antérieur, effectué à Avignon en juillet 1952, fût définitivement effacé. Il n'a pas eu gain de cause et celui-ci devait être diffusé contre son gré durant l'été. Le 21 mars, Vilar, résigné, se félicite «que cette retransmission ait lieu au mois d'août, à un moment où les auditeurs sont moins attentifs ».

On comprend que l'enregistrement de ce Lorenzaccio a toujours été problématique.

11 Une nouvelle et sans doute dernière prise sera effectuée au début de l'année 1955 à Chaillot. Le document qui nous l'apprend est une lettre de Vilar à Nadine Berger (comédienne, épouse de Roger Mollien, entrée au TNP le 8 novembre 1954), datée du 10 janvier 1955: «Une représentation supplémentaire de Lorenzaccio doit être programmée le 29 janvier, en raison de la nécessité de refaire l'enregistrement de l'œuvre (pour le disque) ». Nous avons la confirmation que les techniciens qui réalisent la version phonographique du spectacle pallient les défauts d'une prise en insérant des éléments d'une ou de plusieurs autres prises. 
12 On peut donc se demander ce qui provient précisément de l'enregistrement du 27 novembre 1954 dans le coffret édité en 1956. Il faudrait pour le savoir retrouver les archives techniques du travail final réalisé en studio. Nous connaissons en tout cas l'origine de la discontinuité acoustique et du surgissement illogique de gros plans sonores. Les difficultés rencontrées expliquent sans doute aussi les coupes clairement indiquées sur la jaquette : «Ne contient pas : acte 3 , sc. 5 ; acte 4 , sc. $2,4,7$; acte 5 , sc. 2-5») ainsi que (non indiquée) l'interversion des faces comportant respectivement les scènes 5 et 6 de l'acte 4, bizarrerie reproduite dans les cassettes de 1974. Cependant, si, malgré tous ces défauts, ce disque est encore aujourd'hui prenant, voire addictif, c'est que quelque chose de la nuit Renault y a été conservé, une vérité plus importante que l'appartenance exacte de tous les fragments du montage à l'enregistrement du 27 novembre 1954. Écoutons encore Noémie Fargier dans la fiche d'écoute déjà mentionnée :

«La densité sonore est importante. La volubilité des dialogues laisse peu de place au silence, et les scènes sont entrecoupées et quelquefois accompagnées de musique. De nombreuses scènes de conflit, verbal ou physique, portent l'intensité sonore à son paroxysme. Les spectateurs applaudissent à chaque fin de scène, et après des moments de bravoure (tirade, bataille...).»

13 Les réactions du public et le rythme général font que les commentateurs utilisent parfois l'image du « ring ». L'auditeur est pris dans l'énergie et la tension qu'il peut y avoir lors d'un match de boxe en public. Parmi les très nombreux bravos internes, citons par exemple ceux qui éclatent à la fin du dialogue entre le cardinal Cibo (Jean Vilar) et la Marquise (Christiane Minazzoli), acte I, scène 3 , après une longue réplique passionnée de la Marquise exprimant sa haine contre Alexandre de Médicis, duc de Florence. Sympathie avec les propos de la Marquise? Admiration pour le jeu de la comédienne ? Réaction réflexe à sa sortie (qui suit immédiatement sa tirade) ? Quant aux applaudissements de fin, ils sont nourris, puissants, ponctués de cris, régulièrement réanimés par la musique qui accompagne les saluts, ils semblent ne pas vouloir cesser. Ce qui est assurément demeuré de la représentation du 27 novembre 1954 et autorise à présenter officiellement ce document sonore comme l'enregistrement effectué lors de la «nuit Renault» est la trace de la part active, improvisée, dynamique et dynamisante, du public dans l'événement.

\section{Complémentarité des archives sonores et des archives audiovisuelles : comment rendre compte de Par les villages?}

14 Depuis Filmer le théâtre, un dossier organisé par Denis Bablet en $1981^{12}$, un certain nombre d'études ont paru sur les enregistrements audiovisuels des spectacles de théâtre, de la captation sommaire à la recréation cinématographique en passant par le tournage en direct pour la télévision. On trouve des réflexions de fond sur le sujet dans l'ouvrage Le film de théâtre, paru en $1997^{13}$. Ce qui suit ne prétend pas embrasser l'ensemble de cette grande thématique. La réflexion ne porte que sur une question très précise: l'éventuelle complémentarité des enregistrements audio et des enregistrements audiovisuels d'un même spectacle. Il s'agit d'une première approche et elle s'exerce sur un exemple très particulier : étant donné les relations entretenues par Peter Handke, l'auteur du texte, et par Claude Régy, le metteur en scène du spectacle, 
avec l'image, Par les villages n'est pas un exemple neutre par rapport à mon sujet. Écoutons Claude Régy parlant de ce spectacle au moment où il l'élabore et Peter Handke plus récemment sur la relation du langage à l'image :

"Comme aux origines du théâtre, on retrouve la force du récit: on délivre une matière globale directement de l'auteur au public, dans une mise en scène ouverte, dépliée comme un livre ${ }^{14}$.»

«Ceux qui ne parlent pas [...] doivent ressentir et imaginer avec autant d'intensité que celui qui parle. Tous doivent être celui qui parle ${ }^{15}$. "

«Pour être ouvertes, [les images scéniques] sont le plus souvent minimales. Inspirées par le texte, elles ne le représentent pas. Elles sont d'un ordre virtuel. De la nature des images que nous voyons quand nous lisons ${ }^{16}$."

«Et peut-être n'y a-t-il rien de plus frappant, de plus profond que ces simples motsimages ${ }^{17}$."

15 En refusant (comme d'autres metteurs en scène) le filmage de ses spectacles, alors que les vidéos puis les films de théâtre constituent un ensemble de plus en plus riche et de plus en plus utilisé par les chercheurs, Claude Régy nous incite à formuler une interrogation que notre travail sur la dimension sonore et auditive du théâtre, et plus particulièrement notre travail sur les archives "seulement" sonores des représentations nous a conduits à nous poser : ces archives que nous commençons à mieux connaître sont considérées par Régy comme moins fausses, moins nocives, donc, que les archives dites audiovisuelles. Que pourraient être les éventuelles fonctions de ces dernières dans un dispositif où elles ne seraient pas a priori survalorisées, c'est-àdire implicitement définies comme meilleures parce que plus complètes?

Notre réflexion s'est fondée sur l'écoute ou le visionnage partiel de deux documents :

17 - d'une part, un enregistrement sonore du spectacle conservé à la BnF, dans le fonds Chaillot: deux bandes magnétiques (les supports d'origine ont disparu), reconditionnées sur des bobines de $27 \mathrm{~cm}$ de diamètre, d'une durée respective de $1 \mathrm{~h} 58^{\prime} 55^{\prime \prime}$ et de $2 \mathrm{~h} 04^{\prime} 40^{\prime \prime}$ (en tout : 4 h 03' 40"), numérisées en deux fichiers de format WAV. L'enregistrement n'est pas daté. S'il a été réalisé le même jour que l'entretien figurant dans le dossier comportant aussi deux bandes-son du spectacle, il s'agit de la représentation du 11 décembre 1983. Le spectacle s'est joué dans le "grand théâtre » de Chaillot $^{18}$ du 24 novembre au 23 décembre 1983. La musique est de Tristan Murail. La bande sonore a été réalisée et dirigée par Claude Pavy.

18 Cet enregistrement a été écouté et fiché dans le cadre d'ECHO (l'écoutante, Pascale Caemerbeke, a signalé « un souffle sur la bande»), mais n'a pas fait l'objet d'une étude de notre part. J'avais partiellement écouté l'enregistrement, j'en ai réécouté de larges extraits pour préparer cette contribution;

- d'autre part, une captation audiovisuelle du spectacle conservée aux Archives nationales, dont j'avais visionné une partie avant de décider du présent exercice et de réentendre l'archive audio. Je n'ai pas consulté les supports analogiques d'origine ( 5 cassettes U-Matic couleur BVU [Broadcast Video Umatic: une qualité de résolution un peu meilleure des années 1980] de 60' chacune d'après la description de l'inventaire en ligne). Ceux-ci avaient été copiés au Théâtre national de Chaillot sur des supports numériques: plusieurs copies, plusieurs formats - dont des DVD (des copies basse résolution de la version numérisée) réalisés par l'INA et les fichiers MP4 que j'ai consultés (des copies des fichiers contenus dans ces DVD effectuées par le Théâtre), faisant respectivement 56',61',59',60' et 18', ce qui donne une durée totale de $4 \mathrm{~h} 14^{\prime}$. 
La différence de 9' avec l'enregistrement sonore s'explique en partie par le fait que chaque fichier reprend l'enregistrement un peu en amont de la fin de celui qui précède.

Après avoir écouté trois extraits de l'enregistrement audio (de 45", 59" et 30"), j'ai visionné les extraits correspondants de la captation vidéo (de 44", 1' et 26"). Dans les deux premiers extraits, Hans est interprété par Miloud Khetib, la vieille Femme est interprétée par Muni. Ces extraits, que 20 secondes seulement séparent, se situent à presque 3 heures du début de la représentation. Nova, qui apparait dans le troisième extrait (situé à presque 4 heures du début de la représentation), est interprétée par Claude Degliame. La traduction est de Georges-Arthur Goldschmidt. La musique est de Tristan Murail. La bande sonore a été réalisée et dirigée par Claude Pavy.

Commençons par une remarque sur le document sonore. Malgré le souffle qui affecte toute la durée de la bande magnétique, cet enregistrement, parce qu'il efface ce qu'on appelle les « corps » et leurs topographies scéniques, nous fait éprouver physiquement que ce qu'on appelle "le texte ", ce sont des voix (des timbres, des rythmes, des suites de mouvements ténus), ce sont aussi des mots qui sont des "mots-images", et que voix et mots entendus nous mettent à l'écoute et nous affectent dans nos corps.

Intéressons-nous maintenant au document audiovisuel.

D'abord pour préciser à quel type de document nous avons à faire. Au début de la captation, sans doute effectuée par le régisseur ou un membre du personnel du théâtre, l'image bouge, le cadre n'est pas stable. Quoique les deux premiers extraits soient déjà loin du début du spectacle, les mouvements de caméra sont encore tâtonnants, le travelling avant puis le panoramique vers la droite et le retour au personnage de Hans ont quelque chose de formel, d'artificiel, d'explicatif, ils constituent une sorte de «bruit » visuel et ne contribuent pas du tout à la saisie de ce qui se passe d'important: Hans parle. Alors que le monologue de Nova, qui clôt Par les villages, est fermement filmé. Tout se passe comme si ce spectacle, composé presque uniquement de très longs monologues, avait donné au fur et à mesure au caméraman un geste plus adapté. Cependant, même si le film est de moins en moins maladroit, cette maladresse étant sans doute moins gênante qu'une maîtrise inadéquate, jamais il n'offre une entrée directe dans une expérience qui serait de l'ordre de l'expérience du spectateur. Les documents audio-visuels, puisqu'ils sont "audio " autant que "visuels", devraient pourtant pouvoir nous faire entrer eux aussi dans l'espace quasi tactile de l'audition vocale-verbale évoquée plus haut. Force est de constater qu'ils ne s'y prêtent guère, suscitant automatiquement un tout autre mode d'expérience, supprimant le jeu, le flottement entre l'entendre et le regard qui caractérise l'art du théâtre, un jeu devenu aujourd'hui si vital qu'il est méthodiquement travaillé par de grands créateurs contemporains comme Robert Wilson. La disjonction structurelle des canaux sonore et visuel dans le cas de l'archive que l'on devrait dire « audio-auditive » restaure quelque chose de ce jeu fondamental.

Ce gain majeur semble avoir pour contrepartie la perte totale de ce qui est à voir. Mais c'est faux. Il y a du visuel dans une telle audition. Ce que j'appelle parfois le «quasivisuel», désignant ainsi ce que nous "voyons» lorsque nous écoutons un enregistrement sonore, est sans doute bien plus proche du regard théâtral que l'image fournie par l'audio-visuel, qui en outre nous leurre en nous faisant penser que nous assistons - ou presque - au spectacle, ce que ne saurait faire la captation sonore, indubitablement et presque insolemment lacunaire. "Entrer dans le théâtre par 
l'oreille, c'est mieux comprendre - justement parce qu'on entend sans voir - que le théâtre est un art de l'imagination autant que de la perception ${ }^{19}$. "

Revenons maintenant au document audiovisuel et à ce que peut lui apporter le fait de ne plus être d'emblée ni le plus beau ni le plus fort. Visionné après le document sonore et en relation avec lui, il révèle ses vertus propres.

$\mathrm{Si}$, après avoir écouté le troisième extrait de l'enregistrement sonore, 30 secondes $\mathrm{du}$ long monologue de Nova "parlant» (est-ce exactement "parler»?) du milieu de la musique composée par Tristan Murail, on visionne l'extrait vidéo correspondant, ce qu'on voit sur le plateau est celle qui émettait la voix que chacun/chacune de nous avait préalablement entendue et éprouvée, chacun/chacune de nous ayant été en relation directe avec elle, dans un univers créé par la musique mais polarisé par elle, par cette récitation timbrée, lentement rythmée, très lointaine et très proche. Nous suivons maintenant en l'entendant à nouveau, en continuant à l'éprouver, le jeu de la comédienne, sa performance mémorielle et physique. L'image audiovisuelle restitue le travail théâtral dont nous avons vécu l'effet. Sa fonction essentielle est ici documentaire.

En présentant ces deux exemples, mon objectif est de contribuer au renouvellement de l'usage des archives dans le champ des Études théâtrales par un recours de plus en plus pertinent et fructueux aux enregistrements sonores, eux-mêmes de mieux en mieux documentés. Il est aussi, plus largement, de pointer les limites d'une pensée qui oppose de façon simpliste la vision et l'écoute. Prendre sérieusement en compte la dimension sonore et auditive du théâtre signifie reconsidérer de façon critique les présupposés théoriques qui ont pu la faire oublier.

\section{BIBLIOGRAPHIE}

Le son du théâtre - XIX ${ }^{e}-\mathrm{XXI}$ siècle. Histoire intermédiale d'un lieu d'écoute moderne, Jean-Marc Larrue et M-M Mervant-Roux (dir.), Paris, CNRS éditions, 2016. (Versions papier et numérique)

L'Echo du théâtre 2. La scène parle. Voix, acoustiques et auralités (seconde moitié du XX $X^{e}$ siècle), Jeanne Bovet et Marie-Madeleine Mervant-Roux (dir.), RSL [Revue Sciences/Lettres] 6/2019, Paris, ENS, 2019. https://journals-openedition-org.inshs.bib.cnrs.fr/rsl/1294

«Études théâtrales et Sound Studies. Vers une histoire aurale du théâtre », in L'histoire culturelle au prisme des studies, Sophie Croisy, Lise Guilhamon, François Robinet et Brigitte Rollet (dir.), Diogène, Revue internationale des sciences humaines, 2017/2, $\mathrm{n}^{\circ}$ 258-259-260, Paris, PUF (Presses universitaires de France), juin-septembre-décembre 2017 [2019], p. 154-167.

«Les archives sonores de L'Amante anglaise (Paris, TNP, 1969). L'enregistrement contre la mémoire ? ", in Mary Noonan, Joëlle Pagès-Pindon (dir.), Marguerite Duras. Un théâtre de voix, Leyde (Pays-Bas), Brill, 2018, pp. 75-91. 


\section{NOTES}

1. MERVANT-Roux (Marie-Madeleine), «Peut-on entendre Sarah Bernhardt ? Le piège des archives audio et le besoin de protocoles », dans Sociétés et représentations, $\mathrm{n}^{\circ} 35$, Archives et patrimoines visuels et sonores, dirigé par Évelyne Cohen et Marie-France Chambat-Houillon, Paris, Publications de la Sorbonne, printemps 2013, p. 165-182. DOI : 10.3917/sr.035.0165.

2. DEShaYs (Daniel), Pour une écriture du son, Paris, Klincksieck, "Cinquante questions », 2006, p. 45.

3. «Le son du théâtre ( $\mathrm{XIX}^{\mathrm{e}}-\mathrm{XXI}{ }^{\mathrm{e}}$ siècles) », PICS [Projet international de coopération scientifique] réunissant une équipe du CNRS (ARIAS) et une équipe du CRI [Centre de recherche sur l'intermédialité] de Montréal, et le projet ANR ECHO, l'un et l'autre organisé en collaboration avec le département des Arts du spectacle de la BnF, dont les fonds audio s'inscrivent dans un large ensemble d'autres documents.

4. Selon le terme utilisé par Pierre Schaeffer, qui en faisait un principe méthodologique, un exercice d'hygiène auditive.

5. Fonds du Théâtre national populaire. Direction Jean Vilar, 295AJ/1-295AJ/948.

6. Le texte est de Peter Handke, dans une traduction de Georges-Arthur Goldschmidt.

7. Claude Régy, Les Voies de la création théâtrale, vol. 23, études et témoignages réunis et présentés par Marie-Madeleine Mervant-Roux (ouvrage accompagné d'un DVD-ROM conçu par Éric Vautrin), Paris, CNRS Éditions, coll. Arts du spectacle, 2008.

8. Claude Régy a depuis accepté certaines tentatives de filmage (pour des éléments de spectacles, pas pour des intégrales, à ma connaissance).

9. On peut écouter ces deux extraits à la BnF.

10. "Le micro sous les planches. Répétitions de L'Avare», document INA (diffusion les 9 et 16 novembre 1952).

11. Ces questionnaires ont été proposés de 1952 à 1963. Les spectateurs déposaient les questionnaires remplis au théâtre, dans les boites prévues à cet effet, ou les renvoyaient au TNP par la poste quelques jours plus tard. L'ensemble est conservé aux Archives nationales sous la cote 295AJ/770-295AJ/786. Je remercie Pascale Caemerbeke, assistante à la recherche dans le projet ECHO, d'avoir dépouillé de très nombreux questionnaires et d'avoir ainsi facilité et préparé le travail des chercheurs. Voir son étude: «Le son du TNP de Jean Vilar: les questionnaires des spectateurs (1952-1963) », L'Écho du théâtre. Dynamiques et constructions de la mémoire phonique, XIX ${ }^{e}-\mathrm{XXI}{ }^{e}$ siècles, sous la dir. de Hélène Bouvier et Marion Chénetier-Alev, Revue Sciences/Lettres [En ligne], 5|2017, mis en ligne le 2 octobre 2017, URL: http:// journals.openedition.org/rsl/1191; DOI : 10.4000/rsl.1191.

12. Bablet (Denis) [dir.], Filmer le théâtre, Cahiers Théâtre Louvain, ${ }^{\circ}$ 46, Louvain-la-Neuve, 1981.

13. Picon-Vallin (Béatrice) [dir.], Le film de théâtre, Paris, CNRS Éditions, coll. Arts du spectacle, 1997.

14. Régy (Claude) [à propos de Par les villages] Espaces perdus, Paris, Plon, 1991, p. 90-91.

15. Ibidem, p. 94-95.

16. Ibidem, p. 156.

17. Handke (Peter), La perte de l'image [Der Bildverlust oder Durch die Sierra de Gredos, 2002], traduit de l'allemand par Olivier Le Lay, Paris, Gallimard, 2004.

18. Cette salle n'est plus celle dans laquelle s'était joué Lorenzaccio, détruite et entièrement reconstruite dans les années 1970.

19. Extrait du texte de présentation du site Entendre le théâtre. Un voyage sonore dans le théâtre au XXe siècle (dir. M.-M. Mervant-Roux et Joël Huthwohl). Élaboré par ECHO et réalisé par les éditions multimédia de la BnF, il est en ligne depuis février 2020 sur le site de la BnF (rubrique Classes) :

http://classes.bnf.fr/echo 
C'est cette vision inhabituelle du théâtre que nous avons voulu proposer aux lycéens et à leurs enseignants, aux étudiants (en particulier en études théâtrales), aux élèves des conservatoires d'art dramatique, aux amateurs et pratiquants amateurs de théâtre de toute la francophonie.

\section{RÉSUMÉS}

À partir de deux exemples d'archives sonores écoutées et exploitées dans le cadre du projet ECHO (celles du Lorenzaccio d'Alfred de Musset mis en scène par Gérard Philipe (TNP, Palais de Chaillot, 1952-1954) et celles de Par les villages de Peter Handke mis en scène par Claude Régy ("grand théâtre ", Théâtre national de Chaillot, 1983), nous examinerons comment l'étude des enregistrements audio peut fructueusement s'articuler à celle d'autres types d'archives (manuscrits, imprimés, ou captations audiovisuelles) et comment les fonctions supposées des unes et des autres peuvent s'en trouver redéfinies.

\section{INDEX}

Mots-clés : Administratives (archives) ; Audiovisuelles (archives) ; Philipe (Gérard) ; Régy (Claude) ; Sonores (archives) ; Théâtre national de Chaillot ; TNP (Théâtre national populaire)

\section{AUTEUR}

\section{MARIE-MADELEINE MERVANT-ROUX}

Directrice de recherche émérite au CNRS (UMR THALIM), responsable du projet ANR ECHO 


\title{
Cyrano de Bergerac, mis en scène par Jérôme Savary : le bruit du panache
}

\author{
Océane Djabellah-Peillon
}

1 Le théâtre est, étymologiquement, le lieu où l'on voit. Le théâtre est un lieu, un moment où l'on vit quelque chose. Les artistes sont chargés de transporter le spectateur dans un monde nouveau, une fiction, et ainsi le faire se détacher de la réalité. Tout en marquant, s'il est possible, le spectateur dans sa vie. Pour cela, les spectateurs à la fois observent, écoutent, et réagissent à ce qui se joue devant eux. Ils participent, quoique leur posture soit celle d'une attitude passive. Assister à une représentation est une expérience à part entière, que l'on ne peut jamais véritablement retrouver dans une captation, aussi qualitative soit-elle. Qu'en est-il si l'on ne regarde pas, mais que l'on écoute le théâtre? Comment entamer un travail de recherche à partir de rien d'autre que du son?

2 On a déjà écouté du théâtre avec Marguerite Duras notamment, avec La Musica qui est écrite pour la radio. Mais le tout est pensé pour la radio, n'a pas besoin d'être vu pour être compris et apprécié. Les archives sonores consultées dans le cadre du projet ECHO nous invitent à considérer le son du théâtre non pas en tant que techniciens, musiciens ou comédiens mais en tant que chercheurs. Il s'agit cette fois d'un théâtre qui n'est pas prévu pour la radio, dont le son est la partie sonore d'une archive audiovisuelle. Nous sommes invités à percevoir le théâtre sous un nouvel angle.

3 Comment décrypter une archive en n'utilisant que son ouïe? Ne peut-elle pas partiellement occulter des éléments, en voulant se concentrer sur un aspect particulier?

$4 \quad$ Le son est-il suffisant dans une recherche archivistique? Appréhende-t-on réellement de façon objective une pièce aussi connue et interprétée que Cyrano de Bergerac?

5 Les questionnements surgissant à l'écoute d'une archive sonore sont-ils toujours les mêmes lorsqu'on y ajoute le visuel ? Les bruits qui concernent la salle ne sont-ils pas parasites pour l'archiviste, quoiqu'ils renseignent sur l'accueil du spectacle?

6 L'archive audiovisuelle de Cyrano de Bergerac, texte d'Edmond Rostand mis en scène par Jérôme Savary au Théâtre national de Chaillot durant la saison 1997-1998, se trouve 
dans le fonds du TNC aux Archives nationales sous la cote 20160438/113. La consultation s'est faite sur le site de Fontainebleau, sur un poste informatique, après numérisation des enregistrements originaux: deux cassettes VHS de 180 minutes chacune. La captation a été réalisée par le régisseur du théâtre, pour l'équipe artistique ou un visionnage professionnel, ce qui explique que la bande n'ait pas la qualité d'une production commerciale.

7 La numérisation des deux cassettes VHS a donné quatre fichiers informatiques. Pour décrypter en détail deux des quatre fichiers, 19 heures de consultation ont été nécessaires.

8 Le visuel du spectacle existe donc, qui nous permettra d'aider à la recherche archivistique dans un second temps. Les conditions de consultation des archives sonores sont particulières: dans une salle calme, avec des écouteurs, pour une concentration et une perception des sons et des bruits optimales. Ainsi, il fut aisé de saisir distinctement ce qui se passait sur scène et dans la salle, et de localiser la plupart des bruits.

9 La captation (datée) a été réalisée pendant la représentation du 13 janvier 1998, à la fin de l'exploitation, en présence du public. Le 13 janvier étant un mardi, il s'agit donc d'une représentation donnée en soirée et en semaine. Pas de publics scolaires, ce qui explique le peu de bruits que l'on perçoit du public. Des toux principalement, des applaudissements généreux à la fin des tirades et des monologues célèbres.

10 La surprise, à la première écoute, est la voix de Cyrano. Une voix bien connue, celle de Francis Huster. Et malgré tout, le souvenir de divers Cyrano célèbres hante l'esprit lorsqu'on commence à écouter, davantage encore en n'ayant pas d'image à associer à cette voix. Difficulté majeure pour un acteur qui joue Cyrano : faire oublier les Cyrano précédents. Celui qu'a interprété Gérard Depardieu, quoiqu'il fût cinématographique ; celui de Philippe Torreton au Théâtre de l'odéon aussi. Deux Cyrano pleins de force dans la voix, et dans le corps. La voix de Francis Huster que l'on pourrait qualifier de «nasale », il faut considérer ici que le comédien porte un nez imposant quoiqu'il soit fixé à même la peau. Appendice qui gêne probablement le comédien si ce n'est dans sa diction au moins dans la projection du son.

11 L'écoute nous prive en effet d'un attribut essentiel au personnage : son "panache». Difficile d'écouter Cyrano, quoique le texte soit une œuvre en lui-même, puisqu'il est un personnage que l'on voit et que l'on aime à voir promener son épée et sa poésie, qui nous « touche».

12 Passé ce premier étonnement, d'autre surviennent, liés à la technique cette fois. On perçoit des tremblements de voix, des grésillements aussi, à plusieurs reprises et par vagues, probablement dus à la mauvaise qualité du support, et donc de l'enregistrement, qui n'était pas prévu pour une diffusion télévisuelle. Mais une fois seulement, le spectacle est tronqué. De 1'35" à 1'37" du premier fichier, Francis Huster/ Cyrano se fait couper la parole par la bande-son, comme si un technicien avait mis sur pause l'espace de ces deux secondes. C'est la seule anomalie de cette captation, que l'on peut retenir malgré tout.

La gêne majeure à l'écoute de ces archives se trouve davantage dans les déplacements des comédiens sur scène. Les bruits de pas sont presque omniprésents, jusqu'à le prendre sur les paroles. Ces bruits laissent penser que les comédiens portent, a priori, des talons (et donc des costumes d'époque), et qu'ils évoluent sur du parquet, que l'on 
peut entendre grincer sous leurs pas. Il semble creux par endroits, d'après les nuances des sons produits par les déplacements.

Le parquet sur le plateau est-il d'origine ou a-t-il été installé spécifiquement pour la mise en scène de Jérôme Savary? Pourquoi perçoit-on autant de bruits dans une archive de de théâtre? Entend-on le même son que les spectateurs? Ce son hypertrophié des pas sur le plancher est-il propre à l'archive ? Est-ce une volonté de la mise en scène?

La tirade des cadets de Gascogne, en particulier, met en avant ces bruits de pas (cf. Annexe). Cependant, la diffusion d'un extrait de cette tirade lors des journées d'étude a révélé que les sons ne sont pas perceptibles sans un casque, sans être véritablement immergé dans l'archive. Les bruits de pas sont ici plus agréables à l'écoute que d'autres bruits de pas parasites du spectacle, puisqu'ils sont chorégraphiés sur les paroles de Cyrano/Francis Huster. Cela n'empêche pas quelques pas parasites pendant la tirade, venant du fond de scène, simultanément avec des bruits de grincement de ce qui semble être du parquet. Ainsi, les « ogne » déclenchent des petits pas, un changement d'appui des cadets qui, à l'écoute semblent se situer derrière Cyrano. Sa voix est clairement distincte des autres sons. Quelquefois, le changement d'appui est un saut. À chaque « trou », un cri bref ou des « vivats » des cadets.

16 Les questionnements, survenus lors de la consultation des archives sonores sur le site de Fontainebleau, ont rendu nécessaire de faire appel à d'autres types d'archives. Heureusement, le spectacle vivant laisse, malgré tout, des traces derrière lui. Dont les archives papier. Celles du service technique du TNC ne sont pas encore consultables. Celles du service habillement sont disponibles et consultables par dérogation. Elles ont révélé des informations d'une grande aide dans la résolution des problématiques suscitées par l'écoute des enregistrements.

17 Le dossier « Habillement » est un dossier complet, qui regroupe tous les documents qui ont été nécessaires au spectacle (fiches de mensurations des comédiens, marques de mèches de cheveux, échantillons de tissus, fiches de coordonnées de l'équipe artistique, feuilles de mise, cartes des entreprises contactées pour la location et la fabrication des costumes et des accessoires). La facture de l'entreprise Pompei, enseigne de location et de fabrication de costumes et accessoires, a été particulièrement utile dans la recherche de la source des bruits de pas des comédiens sur la scène. Elle correspond à une commande de fabrication sur mesure de bottes de mousquetaires pour Francis Huster (Cyrano de Bergerac), Alexandre Brasseur et Jean-Marc Thibault. Est également indiquée la fabrication sur-mesure de bottes en agneau pour Cristina Realli (Roxane).

« Mimo » est le bottier qui travaillait dans l'entreprise Pompei dans les années 1990 et qui a accepté un entretien téléphonique. Les bottes en cuir sont de fabrication artisanale. Elles ne sont pas conçues pour faire plus ou moins de bruit sur scène, mais avec des talons propres à ce genre de bottes. Celles fabriquées pour Cristina Realli sont en agneau car les chaussures en cuir pour femmes sont fabriquées avec un matériau plus souple que pour les chaussures d'hommes. La présence de talons explique que l'on entende facilement les pas des comédiens sur la scène. Mais pourquoi résonnent-ils au point de couvrir les voix de temps à autre? Les indications fournies par l'archiviste du Théâtre national de Chaillot, Justine Dilien, ainsi qu'une visite du lieu avec Cédric Bosch, inspecteur de salle, ont permis des combler les dernières lacunes. Au TNC sont conservées les captations des spectacles de cette époque. Aujourd'hui, les plateaux des deux salles du théatre ne sont pas faits ni recouverts de parquet. Cependant, sur les 
vidéos de Cyrano de Bergerac, ainsi que sur celles d'un spectacle joué en alternance, dans la salle Jean Vilar également, Le Voyage de Gulliver, on voit du parquet. L'exploitation du Voyage de Gulliver s'est terminée avant celle de Cyrano de Bergerac. Ce spectacle était joué à la fois avec des comédiens et des marionnettes. Ils évoluaient sur un tapis qui recouvrait presque entièrement le parquet. Malgré ce tapis, qui devait sans doute les éviter, on entend, sur la captation, les bruits des pas. Le parquet avait été posé pour Cyrano de Bergerac, avec probablement la volonté d'un bruit authentique de parquet de théâtre (bruit augmenté par les conditions d'enregistrement).

Revenons à la captation de Cyrano de Bergerac. La caméra se trouvait au niveau de la régie, au fond du public. On peut observer tous les spectateurs avant de voir la scène. Mais on peut aussi distinguer des sortes de "points » sur le bord de la scène. Ce sont les micros, installés à la rampe, pour une prise de son au plus près des comédiens... tellement près qu'ils sont fixés sur ce fameux parquet, captent ainsi directement les pas des acteurs et les tremblements qu'ils provoquent avant de capter leurs paroles. La visite du TNC avec Cédric Bosch nous a permis d'observer qu'aujourd'hui, effectivement, aucun parquet n'est présent sur la scène, ce qui était déjà le cas dans les années 1990. Sous la scène, un espace permet la création de trappes à l'envi. Ce qui explique un bruit plus fort par moments dans l'enregistrement, et par endroits : ces trappes, qui une fois ouvertes formeront les tranchées du siège d'Arras, provoquent un bruit creux lorsqu'un comédien marche dessus.

Le bruit des pas des comédiens sur scène, grossi par les micros, est ponctuellement handicapant pour le chercheur-auditeur de Cyrano de Bergerac, car on n'entend pas toujours tout ce qui se passe. Le public n'entend pas ces bruits et tremblements, sauf au moment de la tirade des Cadets et si l'on tape du pied sur scène. C'est l'archive sonore qui attire l'attention sur cet élément et conduit aux détails techniques de la mise en scène et de la scénographie.

L'archive sonore ne correspond pas fidèlement à un spectacle, ni même à sa captation. Mais elle permet de mettre en avant un ou des éléments de ce spectacle qui ne seraient en aucun cas remarqués si la consultation démarrait par son visionnage. Cette recherche archivistique sonore a permis, d'une part, de découvrir d'autres bruits du théâtre que la voix des comédiens, de s'éloigner $\mathrm{d}$ jeu des comédiens, de la recherche du ton juste, et d'autre part, de prendre sous un autre angle une des caractéristiques du panache de Cyrano de Bergerac, qui n'est pas qu'un nez ou qu'un visage, mais bien un personnage, de pied en cap.

\section{ANNEXES}

\section{Tirade des cadets de Gascogne}

Carbon

Puisque ma compagnie est, je crois, au complet,

Veuillez la présenter au comte, s'il vous plait. 
Cyrano, faisant deux pas vers De Guiche, et montrant les cadets

Ce sont les cadets de Gascogne

De Carbon de Castel-Jaloux ;

Bretteurs et menteurs sans vergogne,

Ce sont les Cadets de Gascogne !

Parlant blason, lambel, bastogne,

Tous plus nobles que des filous, Début enregistrement $n^{\circ} 2$

Ce sont les Cadets de Gascogne Quelques pas sur place

De Carbon de Castel-Jaloux : Pas en groupe à la fin du vers

๔il d'aigle, jambe de cigogne,

Moustache de chat, dents de loups,

Fendant la canaille qui grogne,

CEil d'aigle, jambe de cigogne, Groupe de cadets change de pied d'appui

Ils vont, coiffés d'une vieille vigogne

Dont la plume cache les trous! Cri bref, général des cadets

๔Eil d'aigle, jambe de cigogne, Cri en chour

Moustache de chat, dents de loups!

Perce-Bedaine et Casse-Trogne Cri de quelques cadets

Sont leurs sobriquets les plus doux ;

De gloire leur âme est ivrogne !

Perce-Bedaine et Casse-Trogne, Cri de quelques cadets

Dans tous les endroits où l'on cogne

Ils se donnent des rendez-vous...

Perce-Bedaine et Casse-Trogne Cri de quelques cadets

Sont leurs sobriquets les plus doux !

Voici les cadets de Gascogne « Gascogne » signal pour un pas des cadets

Qui font cocus tous les jaloux!

ô femme, adorable carogne,

Voici les cadets de Gascogne Un pas des cadets à la fin du vers

Que le vieil époux se renfrogne :

Sonnez, clairons! Chantez, coucous !

Voici les cadets de Gascogne Un pas des cadets. Répètent les paroles

Qui font cocus tous les jaloux !

ROSTAND Edmond, Cyrano de Bergerac, 1897, acte II, scène 7. 


\section{RÉSUMÉS}

Décrypter une archive sonore est une expérience à part entière, davantage encore lorsqu'elle est la version sonore d'une représentation théâtrale. Les sons perçus dans ces archives sont non seulement ceux de ce qui se passe sur scène (voix des comédiens, bruits de la technique, musique, bruits de pas sur le plateau) mais aussi ceux de la salle (mouvements, rires, applaudissements, discussions). C'est à partir de questionnements survenus à l'écoute de Cyrano de Bergerac, mis en scène au Théâtre national de Chaillot par Jérôme Savary lors de la saison 1997-1998, que mes recherches archivistiques ont réellement commencé. Croiser ces archives sonores avec des archives papier a été nécessaire pour comprendre certains bruits de scène, leur origine et leur utilité dans le spectacle. L'écoute permet une attention plus grande à des détails non sensibles pour un public "voyant". Les archives viennent combler cette lacune des sens, apporter des réponses. Le croisement des questionnements, des impressions, et enfin des archives permet au chercheur de reconstituer la scène, et de changer sa vision du bruit qui, de parasite, devient révélateur du parti pris de la mise en scène, voire intéressant après quelques recherches et plusieurs écoutes. Le tout est de questionner, pour poser un nouveau regard sur ces archives, pour les faire revivre.

\section{INDEX}

Mots-clés : Théâtre national de Chaillot, Rostand (Edmond), Huster (Francis), Savary (Jérôme), Cyrano de Bergerac

\section{AUTEUR}

\section{OCÉANE DJABELLAH-PEILLON}

Étudiante en Master 1 - Études théâtrales, université de la Sorbonne Nouvelle-Paris 3 


\title{
Sonorités de la censure dans le Macbeth de Matthias Langhoff
}

\author{
Eva Bleibtreu
}

[O]n sépare toujours les sens - ça, c'est notre regard, ça notre oreille -, mais en réalité, la division est artificielle.

[L]e texte est plus que le mot parlé. Le manque de mot aussi, le silence, ou le mouvement peuvent être le texte. Mais cela aussi doit être audible ${ }^{1}$.

1 L'archive à partir de laquelle la présente étude a pu être réalisée est constituée d'un ensemble de captations audiovisuelles d'une des représentations en public du Macbeth mis en scène par Matthias Langhoff, créé au Théâtre national de Chaillot (grande salle) le 15 février 1990 et joué jusqu'au 21 mars de la même année ${ }^{2}$.

2 L'analyse repose sur une approche du spectacle par le son, comme le projet ÉCHO m'a invitée à le faire, et s'attache à étudier la façon dont le metteur en scène élabore un univers sonore propre à produire un discours dramaturgique. Nous verrons que c'est au travers d'une véritable stratification de sons de différentes natures (enregistrements divers, musique jouée en direct, bruitages volontaires et identifiables, bruits a priori anodins) que le metteur en scène parvient à faire ressentir au spectateur ce qui ne saurait être dit.

\section{L'esthétique de Matthias Langhoff}

3 Approcher un spectacle par le biais auditif s'est révélé porteur dans le cas de Matthias Langhoff. Celui-ci pense en effet ses créations avant tout dans leur aspect sonore. Il attribue une fonction dramaturgique importante à la fois aux éléments audibles - ceux que l'on considèrera et remarquera facilement en tant que spectateur (comme la musique) - et aux éléments eux aussi perceptibles, mais qui se laissent vite oublier (comme le son des pas des comédiens évoluant sur scène ou le bruit d'objets qu'ils manipulent). Les seconds, que l'on pourrait considérer comme découlant d'une nécessité physique et non d'une recherche créative, sont en réalité, pour la plupart, prévus par la mise en scène et chargés d'une fonction dramaturgique précise. 


\section{L'archive étudiée}

\section{Les documents (nature, type de support, localisation)}

4 L'archive étudiée provient de la partie audiovisuelle du fonds du Théâtre national de Chaillot conservé aux Archives nationales (versement 20160438). Elle est identifiée par la cote 20160438/78 et classée dans la section "Saison 1989-1990 " (20160438/72-20160438/80) du répertoire dédié à la direction de Jérôme Savary.

5 Les supports analogiques originels sont trois cassettes Betacam SP ${ }^{3}$ PAL Master I, II, III, de 90 minutes chacune. Il s'agit d'un ensemble de captations audiovisuelles de l'une des représentations en public du spectacle. Elles ont été réalisées par la régie du Théâtre de Chaillot. La date n'est pas précisée.

6 Les cassettes ont été numérisées par le Théâtre national de Chaillot. Il en est résulté 1 DVCAM DUB (184 minutes), puis, à l'occasion de la campagne de numérisation de

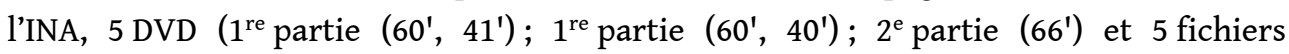
numériques correspondant aux DVD. Ce sont ces fichiers que nous avons consultés pour nos recherches. Les quatre premiers contiennent deux versions différentes de la première partie du spectacle (avant l'entracte) : une version réalisée à partir d'une seule caméra, en plan fixe, avec son mono, et une version montée en post production, filmée avec plusieurs caméras et comportant un son stéréo ${ }^{4}$.

\section{Le contenu de l'archive}

7 Les enregistrements commencent six secondes avant le début du spectacle (on entend les spectateurs discuter entre eux, on en voit quelques-uns s'asseoir) et se terminent une minute après le spectacle (avec les applaudissements, au baisser du rideau). L'entracte ne figure pas dans l'enregistrement.

La balance des couleurs, la luminosité et les contrastes sont standard et restituent une image correcte pour le premier plan, mais peu lisible pour l'arrière-plan. Un phénomène notamment dû au jeu d'éclairage évolutif, avec des zones d'ombres et des zones lumineuses ${ }^{5}$.

Les captations offrent généralement un plan d'ensemble du plateau, souvent visible jusqu'au fond de scène, la prise de vue étant effectuée de la salle, avec quelques plans montrant de plus près un ou plusieurs comédiens

La qualité de l'archive est très bonne. Le spectacle a été enregistré dans son intégralité.

\section{Démarche adoptée pour l'étude sonore de cette archive audiovisuelle}

L'analyse s'est déroulée en plusieurs étapes.

Une première écoute du son seul a été accompagnée d'une prise de notes sous forme d'écrits, mais également de schémas et dessins. Il n'y a pas eu, à ce stade, de manipulation autre que la pause de l'enregistrement. Ainsi nous avons souhaité rendre compte d'un premier relevé des éléments audio qui retenaient notre attention. Un tableau recensant les différents critères de description avait été fourni par le projet 
ECHO et nous a permis d'affiner l'écoute (nature des sons, intensité, localisation spatiale des sons entendus).

13 La durée de l'écoute a été d'environ six heures pour le premier extrait d'une durée de 60 minutes (en raison d'arrêts très fréquents de l'enregistrement pour une prise de note importante) et d'environ trois heures pour les quatre autres extraits (les extraits 3 et 4 n'ont été écoutés que partiellement car ils diffèrent peu des extraits 1 et 2 d'un point de vue sonore; les extraits 2 et 5 ont été écoutés avec une prise de notes simultanée à l'écoute et sans arrêt de l'enregistrement).

Une seconde écoute a ensuite été réalisée avec découverte de la composante visuelle de la captation.

Enfin ont suivi l'écoute et l'analyse répétée d'extraits précis, de 3 à 5 minutes chacun, pour lesquels ont été réalisées des partitions qui permettaient de recenser les différentes couches de sons entendus à un instant $\mathrm{T}$. La méthode employée pour l'écriture de ces partitions a été improvisée, inspirée en grande partie de l'écriture musicale ${ }^{6}$.

\section{Observations découlant de la première écoute}

16 Les résultats du premier relevé ont fait ressortir plusieurs éléments. Il y a tout d'abord dans ce spectacle une grande présence d'éléments sonores, diégétiques et extradiégétiques, avec l'utilisation de musique en direct (des instruments comme le saxophone, les grelots, la flûte traversière), de musique enregistrée (aux sonorités moins claires que celles des instruments en direct), de bruitages enregistrés (sons d'avions mitrailleurs volant à basse altitude, sonnette, téléphone, vent, grillons) et de bruits ou bruitages effectués en direct (on frappe sur une surface en bois pour obtenir le bruit produit par les coups donnés à la porte du château).

Il y a également une forte présence des bruits de pas des comédiens. Celle-ci n'est pas continuelle. Certains déplacements très bruyants se font remarquer plus que d'autres.

Les bruits soudains sont nombreux sur le plateau (chocs et impacts, chutes d'objets, claquements de porte).

Puisque les bruits sur scène étaient considérables, je me suis intéressée à la manière dont ces bruits agissaient sur la compréhension d'une scène et sur le discours dramaturgique en général.

Dans le relevé initial de la première écoute, un motif a retenu mon attention : celui restituant les pulsations rythmiques entendues, traduites par un groupement de trois barres verticales plus ou moins espacées entre elles. Ce motif revenait à deux moments du spectacle.

21 Tout d'abord, il apparaît dans la scène du portier (Acte II, scène 3), lorsqu'on frappe à la porte (ce qui est indiqué dans les didascalies du texte de Shakespeare). Le roi Duncan vient juste d'être tué et, à l'aube, des seigneurs écossais toquent inlassablement à la porte du château de Macbeth dans l'attente que le portier -saoul - vienne leur ouvrir. Cette scène a notamment été analysée par Thomas de Quincey en 1823 dans un court essai resté célèbre nommé "Sur le heurt de la porte dans Macbeth » (« On the Knocking at the Gate in Macbeth»). Dans cet essai, de Quincey explique que ce son a pour fonction dramaturgique de faire ressurgir tout l'effroi du meurtre. La didascalie shakespearienne aurait donc pour fonction de générer un sentiment d'effroi chez le 
spectateur en employant non pas un outil faisant appel à sa compréhension, mais un outil faisant appel à ses sensations.

Le motif des "barres verticales ", c'est-à-dire des coups, est ensuite repris dans une seconde scène où la présence de ceux-ci n'est plus justifiée par le texte. Il s'agit de la première scène de l'Acte IV. C'est cette seconde scène que nous avons choisi d'analyser dans le détail puisque les coups ne trouvaient pas d'explication dans le texte et qu'il s'agissait là d'un parti pris de mise en scène significatif.

Dans cette première scène de l'acte IV, Macbeth vient de monter sur le trône et Banquo, compagnon de Macbeth, se demande si ce dernier n'a pas lui-même tué le roi Duncan pour prendre son titre. Le soliloque de Banquo est alors interrompu par l'entrée du couple Macbeth. Les deux hommes dialoguent sur un mode purement conventionnel et on comprend que chacun maintient les apparences vis-à-vis de l'autre.

\section{Analyse d'un extrait de la mise en scène par l'écoute}

L'extrait sonore auquel nous faisons référence a été étudié à partir du second fichier numérique nommé «TNC_1990_Macbeth_2.MP4 » d'une durée totale de 40 minutes (allant de 3'52" à 4'58").

L'extrait s'ouvre sur un son de cloches d'église évoquant le couronnement de Macbeth. Le dispositif de mise en scène prévoyait des haut-parleurs disséminés dans toute la salle côté public ${ }^{7}$. Le son de cloche diffusé baigne donc l'ensemble de l'espace. Il est assez intense; il ne couvre pas la voix des acteurs mais gêne l'écoute du discours du comédien Gilles Privat (interprète de Banquo).

26 À la diffusion du son de cloche s'ajoute le bruit de coups secs et très forts allant par trois, qui reprennent le son des coups à la porte entendus lors de la scène du portier. Ces coups sont diégétiques puisque le personnage de Banquo les entend et s'en trouve gêné. À mesure que les coups se répètent, Gilles Privat prononce ses répliques en accélérant et décélérant le débit ou en marquant des arrêts en milieu de phrases, comme pour anticiper l'arrivée des coups prochains et se faire entendre malgré l'environnement sonore hostile.

Dans la seconde partie de l'extrait, lorsque Banquo et Macbeth interagissent ensemble, Gilles Privat et Olivier Perrier adoptent des intonations singulières. Olivier Perrier/ Macbeth laisse ses sections de réplique en suspend et n'abaisse pas la voix en fin de phrase. Gilles Privat/Banquo délivre quant à lui sa dernière réplique dans un seul souffle, presque sans variations d'intonation et avec un débit qui dénature le phrasé. La parole est comme forcée des deux côtés. Le spectateur connait alors le fond de la pensée de chacun des deux protagonistes et sait qu'il s'agit là d'un discours cordial en apparences seulement. Le travail de diction des comédiens prévu par la mise en scène traduit les enjeux sous-jacents de la scène.

Enfin, un dernier détail a attiré mon attention. Juste avant l'entrée en scène du couple Macbeth et tandis que le personnage de Banquo est a priori seul, deux derniers coups sont donnés et l'on reste en suspens, cherchant de l'oreille le troisième qui viendrait achever le motif ternaire répété déjà plusieurs fois. Dans l'écoute, puisque l'oreille a été habituée à recevoir le motif en trois temps, lorsque le troisième coup manque, l'oreille continue de le chercher et elle finit par le trouver dans un son similaire de substitution. Ce troisième coup venant clore le motif est généré par le son d'un objet manipulé sur le 
plateau. La mise en scène prévoit en effet qu'Olivier Perrier/Macbeth entre en scène en portant une grosse malle qu'il fait tomber sur une chaise, côté jardin. Le bruit vient compléter les deux coups précédents.

À l'écoute, le son des coups répétés et celui produit par le choc de la malle sont de même intensité. Bien que de natures différentes, ils sont appréciés par l'oreille à un même niveau.

Ainsi, c'est Macbeth qui vient clore le motif sonore. Par ce geste volontaire et très marqué où il laisse tomber la malle sur la chaise, le comédien (mais également le personnage) profère un son semblable à celui des coups qui oppressaient jusqu'alors continuellement la parole du personnage de Banquo. Cette oppression est d'autant plus soulignée que ces coups donnés sont une réelle agression pour l'oreille du spectateur. On ressent physiquement un choc à l'écoute de ce son.

31 L'état émotif que décrivait Thomas de Quincey dans son essai - état émotif dans lequel nous plaçait la scène du portier (juste après le meurtre du roi Duncan) - est restauré ici par ce motif sonore des coups à la porte; Matthias Langhoff réemploie une charge émotionnelle très spécifique. Dans cette seconde scène où le motif sonore n'est pas justifié par la fable, le parti pris de mise en scène semble être de créer un rapprochement avec la scène précédente en exploitant le ressenti épidermique et la mémoire auditive du spectateur.

Banquo, compagnon de bataille de Macbeth, émet des doutes sur ce dernier et le suspecte du régicide attribué aux fils du roi Duncan. Les coups à la porte, répétés sous une forme spectrale, répondent aux tentatives du personnage de faire entendre la vérité- sa vérité - au sujet du roi Macbeth. Tandis qu'il met en mots ses doutes, le personnage de Banquo est oppressé par le son des cloches et le son des coups. Les éléments sonores semblent interagir avec le personnage et prolonger par le son l'oppression politique qui trouvera pour ultime formulation l'assassinat du personnage dans les scènes suivantes.

Ici, la mise en scène nous donne à entendre un prélude mortuaire en employant différents éléments sonores chargés de sens et d'effets. Ce sont là de véritables stimuli nerveux qui éveillaient déjà à l'époque, au sein du spectateur qu'était de Quincey, un effroi qu'il mit lui-même du temps à expliquer.

\section{Conclusion}

L'esthétique de Matthias Langhoff se caractérise par une volonté de produire, en se servant de tous les constituants de la mise en scène, un contre-point au texte, afin d'en révéler toutes les subtilités et possibilités d'interprétations.

Dans le contexte de la scène étudiée, les différents sons intervenant en entrelacs avec les répliques des comédiens agissent comme de véritables partenaires de jeu et sont vecteurs de ressentis émotionnels précis. En employant par deux fois le même motif des coups, Langhoff tisse un lien entre deux scènes pourtant distinctes, proposant des clefs de lecture nouvelles à la pièce. Par ailleurs, le son étant avant tout une vibration, celui des coups opère une agression auditive et transmet au spectateur un choc physique concret. Le soliloque de Banquo est accompagné de cette partition sonore qui donne à entendre et à ressentir l'oppression de plusieurs façons différentes et 
simultanément. C'est au travers de tous ces éléments sonores que la censure de Macbeth sévit.

Prendre la dimension sonore de l'archive comme objet d'étude à part entière et rendre à la partition sonore du spectacle toute son importance dans la lecture dramaturgique de l'œuvre ont révélé des subtilités de mises en scène d'autant plus intéressantes que Matthias Langhoff travaille ses spectacles dans l'idée qu'un son vaut autant qu'un mot ou un geste.

37 Par l'assimilation simultanée des informations données par le texte et de cette expression sonore du non-dit, Langhoff produit un discours dramaturgique permettant au spectateur de ressentir l'intrigue et de l'assimiler à la fois par compréhension logique et par une expérience émotionnelle elle-même d'origine sensorielle.

\section{ANNEXES}

\section{Autres archives disponibles sur la représentation du spectacle au Théâtre national de Chaillot}

Sur ce spectacle, il existe d'autres archives, certaines disponibles à la consultation, comme la centaine de diapositives couleur prises par le photographe Daniel Cande lors de la répétition générale en 1990. Les photographies sont consultables en version numérique sur le site Gallica-BNF ${ }^{8}$ et conservées au département des Arts du spectacle (site Richelieu). On pourra notamment y apprécier le travail de scénographie et les costumes plus en détail, avec un rendu des couleurs précis.

Est également disponible l'affiche-programme du spectacle conservée en deux exemplaires aux Archives nationales?.

Parmi les archives librement communicables, on pourra notamment découvrir aux Archives nationales le budget de production, la conduite de régie, la revue de presse et un ensemble de documents administratifs ${ }^{10}$. 

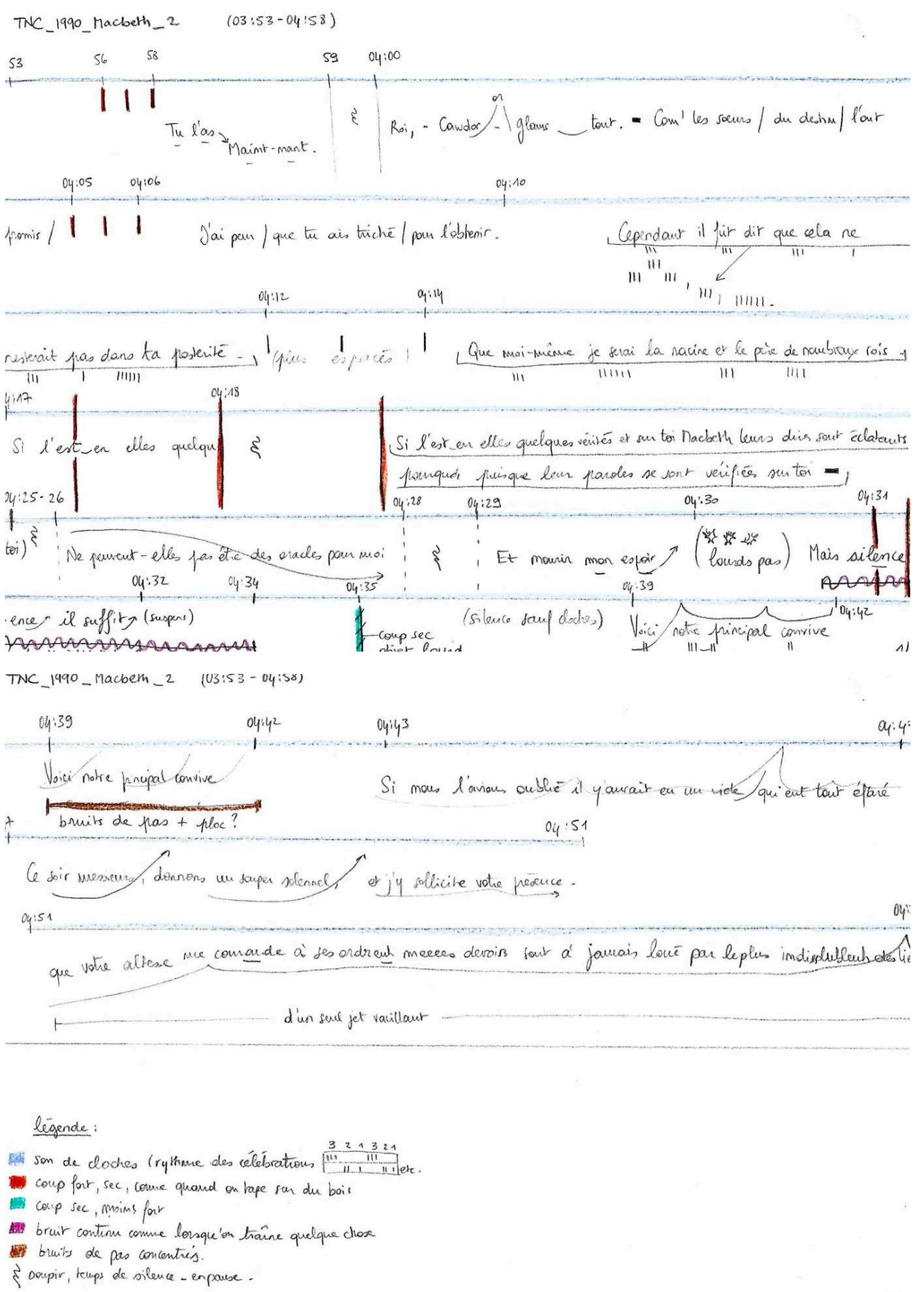

Les couleurs indiquent la nature des sons (le bleu pour le son de cloche, l'orange pour un coup sec donné sur une surface en bois).

Les indications de secondes signalent les arrivées d'un nouvel événement sonore. Ces interventions peuvent être de natures différentes : l'entrée d'un personnage (bruit de pas, nouvelle voix, indication dans le texte), un déplacement sur scène (sons de pas très marqués, variation dans la localisation spatiale de la voix), une séquence musicale (musique en direct ou diffusée), un changement de scène marqué d'un rupture audible (passage d'une ambiance sonore à une autre), une réplique particulièrement travaillée 
d'un point de vue vocal (variation dans le débit, l'intonation et le rythme de la diction) ou encore un silence notable (il y a en a peu dans ce spectacle).

L'intensité vocale des comédiens a été restituée par un tracé de ligne.

Le rythme de l'élocution a été restitué par des groupements de traits correspondant aux différentes syllabes du texte.

\section{NOTES}

1. Matthias Langhoff, extrait de son entretien avec Marie-Madeleine Mervant-Roux, "Sans l'écoute il n'y a pas le texte et sans le texte il n'y a pas le théâtre ", Théâtre(s) en Bretagne, n²0, Presses universitaires de Rennes, 2004, p. 13.

2. Traduction de Jean-Michel Déprats, musique de Hugh Levick, son de Paul Bergel. Le spectacle était coproduit par le Théâtre de Vidy-Lausanne et le Théâtre national de Chaillot.

3. Le Betacam est un format d'enregistrement vidéo professionnel sur bande magnétique développé par Sony à partir de 1982. Les cassettes, dont la bande fait $1 / 2$ pouce de large, comme la VHS, existent en deux tailles: S et L. C'est le premier format analogique professionnel permettant d'enregistrer sur une caméra de manière séparée les signaux de luminance (sur une piste) et de chrominance (sur une autre). La définition est de 300 lignes. En 1987 est développée la Betacam SP qui augmente la définition horizontale à 400 lignes (jusqu'à 700 lignes pour les versions les plus récentes), la fiabilité (bandes métal), la bande passante et la qualité sonore (le nombre de pistes audio passe de 2 à 4). Le Beta SP (pour Superior Performance) devient le standard pour la plupart des chaînes de télévision jusqu'à la fin des années 1990 (définition rédigée à partir de Wikipédia).

4. «La deuxième série de " ${ }^{\text {re }}$ partie" semble être une autre captation, distincte de la première série, avec beaucoup de mouvements de caméra désordonnés, comme un premier repérage.» Précision accompagnant le descriptif de la cote 20160438/78 dans l'inventaire en ligne sur le site des Archives nationales.

5. Pour une description approfondie de la mise en scène, voir ASLAN (Odette), " Macbeth », dans Langhoff, textes réunis et présentés par Odette Aslan, Paris, CNRS Éditions, coll. Arts du spectacle, série Les voies de la création théâtrale, vol. 19, 1994, p. 252-271.

6. Voir en annexe la partition réalisée dans le cadre de l'extrait choisi pour la présente étude.

7. ASLAN (Odette), op. cit., p. 261.

8. https://gallica.bnf.fr/ark:/12148/btv1b9064275m

9. Archives nationales, $20160150 / 1$, pièce 22.

10. Les références de ces sources complémentaires sont indiquées dans l'instrument de recherche du sous-fonds audiovisuel du Théatre national de Chaillot, 20160438 (https:// www.siv.archives-nationales.culture.gouv.fr/siv/IR/FRAN_IR_055964)

\section{RÉSUMÉS}

L'esthétique du metteur en scène allemand Matthias Langhoff se caractérise par la profusion sur scène d'une multitude de stimuli visuels et auditifs qui ne sont pas directement liés à l'intrigue 
du récit présenté. Bruits, paroles et musiques s'entremêlent, se prolongent et se répondent, invitant le spectateur à entrer dans un intime dialogue réflexif avec l'objet scénique où aucune clé de lecture univoque ne lui est offerte. Cette mise en dialogue des éléments génère un discours dramaturgique donnant à entendre ce qui ne saurait être dit.

INDEX

Mots-clés : Théâtre national de Chaillot, Langhoff (Matthias), Macbeth

\section{AUTEUR}

\section{EVA BLEIBTREU}

Étudiante en Master 2 Recherche-Études théâtrales, université de la Sorbonne Nouvelle-Paris 3 


\title{
Les Aventures du petit père lapin : Les signes et les sons
}

\author{
Évangélia Pruvot
}

1 Le théâtre de marionnettes est un théâtre de signes, on y montre des objets que l'on anime et auxquels on prête vie. Ils symbolisent et représentent ce que l'on souhaite montrer, dire, raconter. Les objets marionnettiques signifient quelque chose grâce à un ensemble de signes que chacun doit décoder selon ses propres codes. Par exemple, on est souvent amené à associer des voix aux objets que l'on voit.

Comment écouter un spectacle qui, a priori, est un spectacle visuel?

Dans le cadre du projet ECHO, j'ai d'abord écouté à la Bibliothèque nationale de France l'archive sonore numérisée des Aventures du petit père lapin. Le support d'origine était une cassette U-matic conservée sous la cote NUMAV-045031. Aux Archives nationales j'ai pu écouter puis regarder une deuxième archive, cette fois-ci audio-visuelle, de ce spectacle ${ }^{1}$. Dès les premières minutes d'écoute je me suis rendu compte que les deux archives, celle de la BnF et celles de la BnF n'avaient pas été enregistrées le même jour.

Les Aventures du petit père lapin a été créé en 1985 au Théâtre national de Chaillot par Pierre Blaise, du Théâtre sans toit. C'est un spectacle de 45 minutes qui était joué dans le Grand Foyer.

5 J'ai écouté l'archive sonore de la BnF trois fois en entier en prenant en note les sons au fur et à mesure, ce qui représente quatre à cinq heures d'écoute. Puis j'ai réécouté certains passages plus précisément en notant tous les sons, qu'ils proviennent du public ou de la salle. Enfin j'ai consulté les autres archives concernant ce spectacle à la BnF : des diapositives, des photographies, des coupures de presse, des feuilles de salle ou des documents préparatoires, pour ensuite visionner l'archive conservée par les Archives nationales, plusieurs mois après ma première écoute.

\section{Les sons : musique, bruitages, voix}

6 Le protocole que l'on nous avait demandé de suivre fait qu'à la première écoute, je ne savais absolument pas à quoi m'attendre, je n'avais pas vu d'images, je ne savais pas du 
tout de quoi il était question. Je savais juste que c'était un spectacle de marionnettes. Grâce aux sons que j'ai entendus, j'ai vite compris qu'il y avait, dans les déplacements des personnages, quelque chose de l'ordre d'une course-poursuite. Dans mes notes, j'ai écrit: "peut-être course-poursuite». J'ai pu confirmer cette intuition grâce aux didascalies du texte du spectacle présent dans les archives de la BnF.

7 L'univers sonore est très important dans ce spectacle. La musique prend en charge le rythme et supporte le sens de certaines situations. La musique des courses-poursuites est à l'image du reste des sons : elle est saccadée, vive, assez joyeuse, très bondissante, elle structure toute la pièce et lui donne une teinte malicieuse. Les sonorités de celle-ci sont très reconnaissables.

Les bruitages produisent des images précises, qui peuvent s'avérer incorrectes lorsqu'on les confronte aux vraies images du spectacle, mais qui ne sont pas pour autant complètement détachées du propos général de celui-ci. Ils sont créés synthétiquement, ce sont des sons électroniques, qui évoquent ceux des cartoons. Ils semblent presque faux et ne sont pas réalistes, cependant ils accompagnent l'action et les gags.

9 On peut s'intéresser aux mouvements des marionnettes en écoutant et en regardant un extrait d'une course-poursuite. On entend bien que la musique est rythmée et vive. Grâce à une astuce technique, on s'aperçoit que cette vivacité sonore est accentuée par les déplacements extrêmement rapides des marionnettes. Les marionnettes avaient en effet été fabriquées en trois ou quatre exemplaires. Cela permettait de faire une sortie à une extrémité du castelet et une entrée à l'opposé à la seconde suivante. Les apparitions et les changements de plans pouvaient donc être très rapides. À certains moments, les personnages semblent même se téléporter. Cette technique est utilisée la plupart du temps durant les courses-poursuites où certains déplacements sont accordés avec la musique.

Les voix des quatre interprètes, Pierre Blaise, Grégoire Callies, Jeanne Vitez et Nicolas Vidal, qui ne visent pas à être réalistes, viennent renforcer l'effet produit par la musique synthétique et les sons qui ne sont pas réalistes. Tous les interprètes teintent leur voix et ces voix, même si elles ne sont pas réalistes, nous amènent à imaginer et à visualiser des corps. Or ce spectacle est un spectacle de marionnettes et non de corps. Cela nous reporte au titre que nous avons choisi, au lien entre les signes et les sons dans ce spectacle, et à la question suivante : est-ce que l'écoute d'une archive sonore d'un spectacle de marionnettes est pertinente?

11 A priori, les voix font surgir les corps, mais je me suis demandé si, dans ce cas-là, les voix teintées ne tendent pas à affaiblir l'effet de surgissement des corps produit par l'effet vocal général. Les intonations des voix nous aident à comprendre l'action et donc les mouvements et les déplacements. Ces voix altérées semblent donner chair aux marionnettes et enrayer le surgissement des corps eux-mêmes. Des voix plus réalistes pourraient trop réaffirmer la présence des corps des interprètes. Les textures des voix sont caractérisées pour chacun des personnages et rendent difficile l'identification des interprètes lors de l'écoute de l'archive.

12 Les enfants sont très sensibles aux intonations prises par les différents personnages. Dans les deux enregistrements, on les entend réagir : ils rient beaucoup ou parlent aux marionnettes pour les prévenir d'un danger. On peut se demander quel effet ces voix arrangées provoquent sur eux. Ces jeunes enfants n'ont probablement jamais été au 
théâtre. On peut émettre l'hypothèse que ces voix si particulières les renvoient à une culture dont ils maîtrisent les codes, celle des dessins animés.

Le montage des voix et des images est consubstantiel aux arts de la marionnette. Les spectateurs ont besoin de décoder ce qu'ils voient en agençant les sons avec les objets et en acceptant de faire avec cette bizarrerie. Je me suis demandé comment l'enfant met en relation les images et les sons et aussi comment il les superpose. L'hypothèse que je propose est que l'univers sonore mis en place par les voix, la musique et les bruitages, ainsi que l'univers visuel avec les décors et les marionnettes aux couleurs vives et chatoyantes, emportent les enfants dans l'univers du spectacle.

Ce spectacle de marionnettes à gaine tient véritablement le spectateur par les oreilles et par la vue. Le son est très travaillé et l'une des premières choses que j'ai écrites dans mon cahier lors de ma première écoute est : "ça fonctionne ». Par "ça fonctionne ", j'entends que l'on saisit ce qui se produit au plateau et que l'on est pris par la trame narrative, un peu comme si l'on nous racontait une histoire avant d'aller dormir. Les images apparaissent dans notre esprit et sont immédiatement très concrètes.

\section{L'expérience ECHO}

Faire cette expérience a tout changé dans ma manière d'aller voir un spectacle et de l'écouter. Je fais beaucoup plus attention au son que je ne le faisais auparavant. Je travaille sur les arts de la marionnette et dans le cadre de mes recherches il m'arrive maintenant de ne pas regarder l'image mais simplement d'écouter, afin de visualiser ce que j'entends.

16 Cette expérience m'a fait me questionner sur le rapport entre les sons et les images et sur l'importance du son dans les arts de la marionnette. Spontanément on se dit que la marionnette, c'est visuel parce que c'est un objet avec une certaine qualité plastique, quelque chose que l'on peut toucher. Et pourtant la conclusion que l'on semble pouvoir tirer de l'écoute de ce spectacle de marionnettes à gaine est que la marionnette est un art autant sonore que visuel.

Grégoire Cailles, qui a joué dans ce spectacle, $\operatorname{dit}^{2}$ que, lorsqu'il crée un spectacle de marionnettes, il enregistre, puis écoute le spectacle pour pouvoir ajuster le rythme. Il semblerait que le rythme soit important pour les marionnettes et qu'il ne s'improvise pas. On entend si cela fonctionne ou pas à l'oreille. Grégoire le dit, parfois c'est à une ou deux secondes près qu'on arrive à entendre si le rythme est juste ou s'il ne l'est pas. Il ajoute qu'avec l'image, on est troublé, que l'on n'arrive plus à bien entendre. On est emporté par elle et on ne peut plus déterminer si le rythme est juste car elle parasite notre écoute.

18 À propos du lien entre le son et les images, François Lazaro, marionnettiste, du Clastic Théâtre, raconte une histoire ${ }^{3}$ : il explique que, pour un spectacle, ils avaient besoin du bruit d'une claque, qu'ils ont passé beaucoup d'heures à enregistrer d'abord le son d'une vraie claque, puis de mains qu'on tape entre elles, puis le son d'une claque sur une jambe, un bras, un ventre, et que, lorsqu'ils écoutaient ces sons, aucun ne ressemblait au bruit que fait une claque quand on l'entend directement. Il explique qu'ils ont fini par taper partout où ils le pouvaient, sur une table, un tabouret, un mur avec un verre, un livre, avec tout ce qu'ils avaient sous la main. Pour que finalement 
aucun des sons enregistrés ne semble juste. Il raconte qu'en essayant d'écouter les sons avec l'image, tous les sons fonctionnaient et avaient l'air d'être un bruit de claque.

Cette histoire me fait penser que la force de captation des images est très grande et que si l'on est habitué à voir des images, on est très mal habitué à véritablement écouter les sons qui nous entourent. L'expérience de François Lazaro montre que c'est grâce à l'image que le son a pris sens. Ce n'est qu'avec l'image qu'il est devenu significatif. Cependant, mon expérience avec Les Aventures du petit père lapin a semblé montrer l'inverse ou du moins quelque chose de plus nuancé.

La conclusion que j'en ai tiré est que si l'archive sonore de ce spectacle est facile à écouter, c'est que le spectacle renoue avec deux traditions qui facilitent l'écoute. D'une part, il renoue avec la tradition marionnettique. Dans les années 1980, les marionnettistes sortent de derrière les castelets, ils arrêtent de jouer cachés et ils commencent à expérimenter la manipulation à vue ou le corps-castelet ${ }^{4}$. Or Les Aventures du petit père lapin est une mise en scène avec castelet. Les codes de la marionnette à gaine, qui sont plutôt respectés, en font un spectacle très rythmé. La deuxième tradition avec laquelle renoue ce spectacle est celle de la tradition orale, car Les Aventures du petit père lapin est inspiré de contes et légendes qui étaient transmis oralement dans les plantations du sud des États-Unis 5 . Ces histoires ont probablement évolué au fil du temps, tant dans les mots utilisés pour raconter les péripéties des animaux que dans le choix des péripéties elles-mêmes, ainsi que dans la structure rythmique de chaque histoire, afin de pouvoir créer au mieux des images très précises. On peut faire l'hypothèse que les marionnettes de cette mise en scène viennent peutêtre seulement proposer une version des images qui sont traditionnellement proposées par ces contes.

21 Le fait que ce spectacle soit inspiré de contes oraux et qu'il s'inscrive dans une tradition marionnettique pourrait expliquer pourquoi les sons seuls fonctionnent si bien et pourquoi l'archive sonore semble presque se suffire à elle-même.

Les Aventures du petit père lapin est un spectacle destiné à un public de plus de cinq ans. Le décor est une forêt dans laquelle des animaux se jouent des tours. Le spectacle raconte leurs péripéties en une succession de courtes scènes. Il n'y a pas vraiment de progression, ils se tendent des pièges dans une scène, puis un autre dans la suivante et chaque scène se termine par une course-poursuite sur un thème musical qui revient à chaque course-poursuite. La structure est la suivante: scène, course-poursuite en musique, scène, course-poursuite en musique, etc.

\section{NOTES}

1. Les Aventures du petit père lapin de Pierre Blaise le 5 juin 1985 au Théâtre national de Chaillot (Archives nationales, 20160438/46).

2. Entretien avec Grégoire Callies réalisé le 5 décembre 2018 au Théâtre Roublot-Pilier des Anges à Fontenay-sous-Bois.

3. Entretien avec François Lazaro réalisé le 7 juin 2018 au Clastic Théâtre à Clichy. 
4. Entretien avec Pierre Blaise réalisé le 12 décembre 2018 au Théâtre aux Mains Nues à Paris.

5. http://theatresanstoit.fr/spectacles/les-aventures-du-petit-pere-lapin/ [consulté le 27 octobre 2018].

\section{RÉSUMÉS}

Les Aventures du petit père lapin par le Théâtre sans Toit, joué en 1985 au théâtre National de Chaillot, raconte les péripéties des animaux de la forêt et des pièges malicieux qu'ils se tendent les uns aux autres. La pièce est structurée par des courses-poursuites qui séparent les différents moments du spectacle. Les marionnettes circulent dans le décor disposé en haut du castelet sur plusieurs plans. Les sons - qu'ils soient des bruitages, des voix ou de la musique - ont une place centrale dans l'esthétique de ce spectacle mais aussi dans la compréhension des signes qui le composent. Les arts de la marionnette sont un théâtre de signes et l'écoute de l'archive permet de prendre conscience de l'importance de l'environnement sonore d'un spectacle marionnettique.

\section{INDEX}

Mots-clés : Théâtre national de Chaillot, marionnette, Blaise (Pierre), Les Aventures du petit père lapin

\section{AUTEUR}

\section{ÉVANGÉLIA PRUVOT}

Étudiante en Master 1 - Études théâtrales, université de la Sorbonne Nouvelle-Paris 3 


\title{
Les archives d'architecture : comprendre ce que fut l'outil du TNP à Chaillot
}

\author{
Sandrine Dubouilh
}

1 En 1952, le Théâtre national populaire désormais sous la direction de Jean Vilar se réinstalle à Chaillot. Déjà la première salle des fêtes, réalisée par Davioud et Bourdais en 1878, avait accueilli en 1920 le tout premier Théâtre national populaire, plaçant Firmin Gémier aux commandes d'un improbable vaisseau de presque 5000 places, conçu essentiellement pour les concerts. La salle reconstruite en 1937 à l'occasion d'une nouvelle exposition universelle ne sera guère plus favorable au théâtre. Dans un vaste rectangle plaçant les spectateurs les plus éloignés à quarante et un mètres de la scène, est aménagé un parterre gradiné surmonté d'un balcon pouvant accueillir une capacité totale de 2774 spectateurs, dans une configuration frontale encore peu répandue alors.

Bien qu'il s'exprime peu sur l'architecture de la salle de spectacle du Trocadéro, on perçoit dans certains commentaires de Jean Vilar combien l'exploitation de celle-ci a pu être difficile. Ainsi, lors d'une tournée en Allemagne, Vilar consigne dans ses notes ses impressions sur le théâtre de Krefeld fraîchement reconstruit et dont il admire les vastes espaces : «Le jour inonde les couloirs, les loges, les bureaux et le sous-marinier que je suis (U-boat "Chaillot" immobilisé par 25 mètres de fond) se promène avec délice dans cette lumière ${ }^{1}$ ». Car la décision de raser le corps central de l'ancien palais, construit par Davioud et Bourdais pour dégager la perspective sur la Tour Eiffel et créer ainsi le belvédère que nous apprécions encore aujourd'hui, implique la reconstruction de la salle de spectacles sous la dalle du Trocadéro.

Mais sa situation en sous-sol n'est pas le seul problème que pose l'installation au Palais de Chaillot d'un théâtre permanent. Certes, Jean Vilar a prouvé à Avignon sa maitrise d'un lieu difficile, non conçu pour accueillir des spectacles². Mais pour une troupe installée quotidiennement dans les murs, la relation avec le lieu ne se limite pas à l'outil de création et concerne bien l'ensemble du bâtiment. Or, que l'on se penche sur la première ou la seconde salle, ni l'une ni l'autre n'a été pensée pour accueillir une exploitation de ce type. Gémier occupera la salle des fêtes quarante-deux ans après son 
inauguration, Vilar la salle de spectacle, quinze ans après son achèvement. Ces délais suffisent à nous mettre sur la voie des écarts entre usages projetés et réels, source de très nombreux désagréments pour les occupants des lieux. Que pouvons-nous retracer de cette histoire à partir des archives à notre disposition et que pouvons-nous espérer saisir des relations entre le lieu et ses occupants à partir de ces sources? Inversement, qu'a apporté à notre connaissance de l'histoire architecturale de ce site l'intérêt porté à celle de ses occupants? Tels sont les enjeux des recherches menées depuis 2014 sur la salle de 1937 dans le cadre du programme de recherche $\mathrm{ECHO}^{3}$ et dont cet article va esquisser quelques éléments de l'enquête et des résultats.

Les archives d'architecture à notre disposition concernent aussi bien des documents iconographiques, très souvent incomplets, que des documents textuels, indispensables à la compréhension des premiers. Ces textes sont variés, émanant de la maitrise d'ouvrage (la commande) et de la maîtrise d'œuvre (les architectes), sans oublier les dossiers de marché avec les entreprises. D'autres sources textuelles à prendre en compte sont celles laissées par les commentateurs que ce soit la presse ou les usagers. Tout en regrettant le caractère souvent lacunaire des traces, y compris pour un monument aussi célèbre que le Palais de Chaillot, nous nous rangerons ici aux propos de Siegfried Giedion affirmant dans Espace, temps, architecture que "L'architecture est une indication infaillible de ce qui s'est véritablement passé à une époque donnée ${ }^{4}$ ». En effet, si le recoupement de ces sources nous permet d'approcher la compréhension de la genèse de ces deux salles et de documenter la vie du TNP dans ses murs, les salles du Palais de Chaillot ont aussi la particularité de traduire certaines aspirations fortes de leur temps. La salle des fêtes de 1878 est une expérience, unique sur notre sol, de grande salle populaire. Celle de 1937 explore les contraintes de la polyvalence. Et bien qu'on ne l'étudie pas ici, la salle transformable de 1975, réalisée par Valentin Fabre et Jean Perrottet, s'inscrit au cœur des réflexions sur l'expérimentation scénographique. Chacune d'entre elle a pour ainsi dire la valeur d'un prototype, explorant des configurations encore marginales, que d'autres salles réaliseront ultérieurement avec beaucoup plus d'efficacité dans des formats plus modestes.

\section{La salle des fêtes de 1878 au prisme des ambitions du théâtre populaire}

Lorsque la décision de raser l'ancienne salle des fêtes est prise en 1935, une pétition est lancée pour venir au secours de l'édifice réalisé par Davioud et Bourdais. Pourtant, les critiques se sont rapidement déchaînées contre ce palais que César Daly assimilera à " une toile de fond ", un "décor ${ }^{5}$ ", et Huysmans à " un ventre de femme hydropique couchée la tête en bas, élevant en l'air de maigres jambes chaussées de bas à jour et de mules d'or ${ }^{6}$ ». Quant à la salle des fêtes elle-même, on peine à trouver des éloges la concernant. Il est cependant très difficile d'oublier cette première salle quand on étudie la genèse de celle de 1937, tant sur le plan de l'architecture que celui de son utilisation par le premier Théatre Populaire. Mais il est surtout impossible de couper ces deux réalisations des contextes très particuliers qui les ont vu naître, à savoir les deux expositions universelles. Les premiers documents à considérer pour comprendre leur genèse et incidemment les limites de leur exploitation, ce sont donc ceux relatifs à la commande. 
Il n'a jamais été question, aussi bien en 1878 qu'en 1937, d'édifier un théâtre. Il suffit de se plonger un peu dans les articles de presse de 1876 pour comprendre combien l'exposition universelle prévue deux ans plus tard, dédiée aux progrès de l'industrie, est un choix politique. Le contexte est très tendu, nous sommes entre deux guerres, mais les canons sont encore chauds et près à servir à la frontière Est de l'Allemagne. L'Empire de Bismarck perçoit la manifestation française comme une provocation : estce bien le moment de convoquer toute la planète à faire la démonstration de sa puissance industrielle? La jeune troisième république française n'a-t-elle pas mieux à faire ? Les articles de la presse allemande témoignent sans détour de cette légèreté qui frôle selon eux l'indécence :

Une exposition universelle présente une signification lorsqu'elle est la résultante d'une longue période de paix, d'un travail producteur non interrompu, du bien-être général et de la concorde des peuples. Quand la faillite ravage le monde des affaires, quand la méfiance arrête toutes les transactions, quand les fabriques sont fermées et l'industrie dans la ruine, quand une crise politique terrible tient en suspens le monde entier, le goût et l'ardeur manquent pour de semblables fêtes ${ }^{7}$.

7 En France même, la tenue ou non de cette exposition est un sujet de polémiques et d'attaques plus ou moins directes. Les tensions intérieures restent vives et les Bonapartistes aiment faire circuler dans leurs journaux le bruit de l'annulation de l'exposition. Les animosités vont bon train entre presse républicaine et presse conservatrice. Les partisans de l'ancien régime ne manquent pas de rappeler le succès de l'exposition universelle de 1867 et prédisent à celle de 1878 un échec à la hauteur de l'inconséquence des nouveaux dirigeants: "Nous autres conservateurs, nous comprenons le patriotisme d'une façon toute autre que les républicains et qui doit leur paraître singulière. Nous voulons que tout ce que fait la France soit à l'abri des moqueries, voire même de la critique de l'étranger. Nous voulons que notre génie national apparaisse toujours à l'univers, comme le flambeau du progrès et de la civilisation ${ }^{8}$ ». Néanmoins, l'exposition universelle sera bel et bien un succès, avec une fréquentation légèrement supérieure à la précédente ${ }^{9}$ et marquée par quelques réalisations architecturales remarquables, telles que le Palais du Champ-de-Mars, triomphe de l'architecture métallique. Entre ce bâtiment et le Trocadéro se déploient les pavillons exotiques et le bâtiment de Davioud et Bourdais lui-même n'était pas supposé rester en place. Il doit sa pérennité à la médiocrité du sous-sol obligeant à des fondations solides, favorables à la construction d'un bâtiment en dur. Dans la précipitation de cette commande où les architectes n'ont eu que vingt-quatre jours pour rendre leurs esquisses, le pavillon central du Trocadéro abritant la salle des fêtes devient l'opportunité de réaliser l'Orphéon voulu par Haussmann et étudié par Gabriel Davioud pour la place du Château-d'Eau, actuelle place de la République. De ce projet idéal, réduit de moitié, subsiste donc un grand auditorium de 4665 places réparties en parterre, loges couvertes, loges découvertes, amphithéâtre et tribunes dans un plan circulaire, adossé à une scène atrophiée. Les spectateurs les plus éloignés, situés dans les places les plus hautes, sous les baies, sont à plus de soixante-dix mètres de la scène ; le diamètre de l'auditorium atteint quant à lui les cinquante-trois mètres. Ce gigantisme est emblématique des réflexions de l'époque sur une salle populaire qui permettrait d'accueillir toute la population de Paris, un lieu de paix civile bien nécessaire après les violences de la Commune. Plusieurs projets non réalisés en étudient les principes, à l'instar de celui d'Edmond Viel, représentatif de ce courant de pensée : 
Créer un opéra populaire! Répandre au sein des masses ces trésors qui leur ont été si longtemps interdits ; agrandir, élever les intelligences, ouvrir de vastes horizons, faire naître les plus pures, les plus nobles jouissances au profit des déshérités de l'art ; quelle belle aspiration, allez-vous dire, mais aussi quelle folie utopique ${ }^{10}$ !

L'Orphéon de Gabriel Davioud, dont les premières esquisses remontent à $1863^{11}$, est ainsi présenté comme un projet d'opéra populaire par Alphonse Gosset dans son Traité de construction des théâtres, paru en 1886. Gosset note que la salle réalisée au Trocadéro ne reprend pas toutes les caractéristiques de ce projet de papier pensé pour 9000 spectateurs, notamment la voûte, alors soigneusement étudiée par Davioud et Bourdais selon un profil plus écrasé, suivant une ligne parabolique supposée très favorable à l'acoustique ${ }^{12}$. Gosset souligne également le choix d'une salle en gradins, « de la forme demandée par $M$. Lachèz, très développée en face de la scène et très resserrée au cadre, de façon à bien retenir et répercuter sur les spectateurs les sons émis à l'orchestre et au proscenium ${ }^{13}$ ». Théodore Lachèz, dans son Acoustique et optique des salles de réunion publique ${ }^{14}$, préconise en effet, premièrement pour le confort visuel, que les spectateurs soient assis en face de la scène sur des gradins, une disposition que bien des contemporains perçoivent alors comme plus favorable sans pouvoir vraiment s'y résoudre. Notant l'exemple du Festspielhaus de Bayreuth, dont le plan trapézoïdal est alors une innovation, Gosset traduit bien cette réserve : "mais nous doutons que jamais nos yeux, peut-être trop mondains, s'habituent à passer trois ou quatre heures entre les deux longs murs de cet amphithéâtre, du reste grandiose ${ }^{15}$ ».

\section{Le premier Théâtre National Populaire dans ses murs ; les comptes rendus d'exploitation}

9 La configuration du Palais du Trocadéro, contemporain à quelques années près de l'opéra Garnier, a donc quelque chose d'inédit, en phase avec les aspirations de son temps. Si le programme du concours de l'exposition universelle ne valorise pas cette vocation de salle populaire, celle-ci s'impose quelques années plus tard. On en trouve trace lors de la séance du 26 décembre 1903 au Sénat où se prépare le budget de 1904. Le rapporteur du budget évoque les aménagements nécessaires à la salle du Trocadéro " en vue d'y donner des concerts véritablement populaires ${ }^{16}$ ". Le rapporteur note que "la salle du Trocadéro se prête parfaitement à cet objet", mais que des " améliorations " sont nécessaires, notamment sur le plan des conforts acoustiques et thermiques, deux écueils qui grèveront lourdement son exploitation, notamment par le Théâtre populaire. La rétrocession du bâtiment à la Ville de Paris une fois l'exposition achevée pose de fait problème sur cette part des investissements à consentir par l'État, peu concerné par la question du chauffage puisque l'exposition se déroulera durant la saison estivale. Les architectes optent pour une solution supposée réduire les besoins en combustible. Le renouvellement de l'air est assuré par des cheminées situées de chaque côté de la conque d'orchestre. L'air pur est puisé dans les carrières en sous-sol, frais en été et chauffé en hiver par les piliers des carrières, et conduit en haut de la voûte. L'air vicié est extrait au pied de chaque fauteuil par un ingénieux système intégré à la confection de ceux-ci ${ }^{17}$. À cela s'ajoutent deux imposantes chaudières situées dans les sous-sols ${ }^{18}$. Malgré cela, l'utilisation du Palais avant son attribution au Théâtre national populaire reste très limitée en dehors des périodes estivales. Quant à l'acoustique, le sujet est tellement problématique qu'il suscite, d'une part, l'intervention spontanée de Gustave Lyon, ingénieur polytechnicien, d'autre part, la 
constitution d'une commission sur l'acoustique chargée de suivre les préconisations de Lyon et de veiller à leur mise en œuvre ${ }^{19}$. Lorsque Firmin Gémier s'installe au Trocadéro, Gustave Lyon a procédé à des transformations dans la salle, ajoutant des étoffes absorbantes sur les parois et devant les baies, dont il se dit très satisfait. On mesure cependant sans peine combien cette salle conçue pour les concerts d'orgue, dépourvue de scène et d'équipements scéniques, offrant des proportions inhabituelles pour le théâtre clos, a dû être peu adéquate à cet usage. La première saison du TNP en 1920-21 accomplit pleinement les attentes du cahier des charges, programmant plus de 120 séances. Mais cet usage intensif attire les réserves de la Préfecture de police s'opposant en juin 1921 à la poursuite de ses activités « dans l'état actuel de la scène du Trocadéro, scène rudimentaire conçue pour des concerts et des réunions ne comportant qu'un plateau à peu près nu, et non pour des manifestations théâtrales qui nécessitent des éclairages compliqués, des décors en étoffes et autres matières inflammables ${ }^{20} »$. La construction d'une véritable scène et de ses dépendances a donc été la première nécessité pour le Théâtre national populaire, ce que rappelle Gémier dans son compte rendu d'exploitation de 1929 revenant sur ses premières années où il " a dû agencer complètement la salle du Trocadéro où rien n'a été prévu pour une exploitation de ce genre", des transformations qui concernent «la construction et l'aménagement des loges d'artistes, rideaux, châssis et décors de scène, l'installation cinématographique ${ }^{21} »$. Gémier demandera longtemps la construction d'un magasin de décors où stocker son matériel.

Mais les questions de confort concernent tous les usagers. Le compte rendu d'exploitation de l'année 1930, égrène une fois encore la liste de tous ces ajustements nécessaires et demandés chaque année, allant de "l'établissement en sous-sol de lavabos et w.cl. en nombre suffisants ${ }^{22}$ ", à la construction d'une galerie couverte pour permettre aux spectateurs des tribunes, les moins fortunés, d'être à l'abri pour gagner leurs places, sans oublier l'éventualité d'étendre à la salle de spectacle le chauffage au mazout dont bénéficient les musées situés dans les ailes adjacentes. Au manque de confort en général, s'ajoutent aussi les difficultés de gestion de ce palais aux concessions multiples. Ainsi, le compte rendu de l'année 1922 expose les paradoxes de cette situation qui prive le TNP de la gestion du buffet-bar et des sanitaires : " Il paraît difficilement admissible qu'un théâtre populaire ne possède pas de W.C. gratuits ${ }^{23}$ », un état de fait entraînant les plaintes de spectateurs, tandis que la disposition des locaux complique le partage de gestion des espaces et leur surveillance, le TNP déplorant de nombreux vols. Évoquer le TNP dans ses murs, c'est donc bien prendre la mesure de cet ensemble.

11 Jusqu'aux mois précédant la décision de sa démolition, le pavillon central du Palais du Trocadéro fera l'objet de travaux d'amélioration et de consolidation. Mais en dépit de sa disparition, l'histoire de cette première expérience du Théâtre national populaire dans les murs du Palais va laisser des séquelles sur la nouvelle salle de 1937.

\section{Un autre regard sur la genèse de la salle de 1937 par l'étude des archives d'architecture}

12 La transformation du Palais du Trocadéro se profile à l'occasion de l'exposition universelle des «Arts et techniques appliqués à la vie moderne» de 1937. On notera que le contexte international n'est guère plus pacifié que celui de 1878, mais, ici, l'enjeu 
architectural s'impose sur les questions diplomatiques. Comme dit plus haut, la démolition du palais de Davioud et Bourdais fait des remous et le projet des auteurs de cette proposition, Carlu, Boileau et Azéma, suscite des réserves des milieux professionnels eux-mêmes ${ }^{24}$. Les archives de Jacques Carlu recèlent un document à la fois riche par les informations qu'il nous délivre et pour ce qu'il traduit de l'intérêt porté par l'architecte à l'opinion publique: quarante feuillets recensent ainsi les articles favorables et défavorables au projet pour les années 1935-36 25. Ce recensement concerne la presse quotidienne et spécialisée en France et à l'étranger. Aux 630 articles jugeant positivement le projet, s'opposent les 235 qui le regrettent ou le condamnent. Bien que les grandes étapes de cette décision aient été plusieurs fois exposées dans les ouvrages consacrés à l'histoire de cette opération ${ }^{26}$, il n'est pas si aisé de comprendre à quel moment le projet initial de camouflage intégral du Trocadéro, objet du concours d'idée de 1935, a basculé vers la démolition du pavillon central abritant la salle des fêtes. Sans revenir sur les phases préliminaires de ce concours et les réponses apportées, nous allons nous attacher plus particulièrement au cas de la salle de spectacles elle-même. Sommairement évoquée, l'histoire semble assez simple : Carlu, Boileau et Azéma avaient été désignés lauréats du projet de camouflage avec leur devise baptisée «Bleu, blanc, rouge ». Cet appel à concurrence comportait deux parties, une sur le bâtiment dans son ensemble, l'autre sur la salle de spectacle. Sur cette seconde partie, la devise «Phi Phi » d'Édouard et Jean Niermans, associés à Moyne et Tantot, attire l'attention. Leur projet prévoit, conformément aux recommandations du concours, de moderniser la salle dont l'intérieur serait entièrement rhabillé, cela ayant pour effet de masquer les grandes baies intérieures et incidemment de supprimer les places des tribunes. En revanche, la très grande hauteur sous la voûte (33 mètres) est conservée et la scène, bien qu'agrandie, serait restée selon toute probabilité inconfortable. Lorsque la décision de reconstruire la salle est prise, les frères Niermans sont appelés pour cette opération. Cependant, lorsqu'on se penche sur les archives de la période 1935-1936, on se rend compte que Carlu, Boileau et Azéma ont fait bien plus que de définir pour leurs confrères les données géométriques du projet, autrement dit sa localisation en sous-sol, ses dimensions et ses accès. Le principe global d'une salle en gradins surmontée d'un balcon est établi par eux dès 1935. Un calque au fusain émanant de l'atelier de Carlu, daté du 26 octobre $1935^{27}$, montre une vue de la salle avec le public assis face à la scène et un cadre de scène monumental qui n'est pas sans évoquer celui qui sera effectivement réalisé. On peut aussi rapprocher le dessin de la salle de Chaillot de l'auditorium de l'Eatons College de Toronto, réalisé par Carlu six ans auparavant, où l'on trouve, dans un format beaucoup plus modeste, une organisation semblable de l'espace. Il n'est donc pas évident d'établir quelles furent les marges de manœuvre d'Édouard et Jean Niermans quant à la conception de la nouvelle salle de spectacles. On notera ici combien les sources journalistiques peuvent également nous induire en erreur. Le programme du concours demandait explicitement que la jauge de la salle des fêtes fût conservée. Les articles de presse font état successivement d'une salle de 4000 places puis de 3500 et, finalement, la salle réalisée en comptera 2774 . L'écart est considérable. Mais si l'on compare les plans publiés par Le Génie Civil en mai 1938, donc à un moment où la construction est très avancée, on s'aperçoit que ces plans ne sont pas ceux du bâtiment alors presque achevé. Le balcon y est beaucoup plus profond et cette phase du dessin correspond sans doute à une étape du projet où la jauge envisagée était en effet plus grande que la salle définitive. Tenter une approche 
génétique par l'iconographie ne va donc pas toujours de soi tant il est difficile de rassembler et d'ordonner toutes les phases d'un même projet.

13 Mais le recoupement des plans, s'il peut nous faire douter du rôle d'Édouard et Jean Niermans quant à la disposition du public et au caractère général de la future salle, nous montre au contraire leur importance sur la partie scénographique et acoustique où la grande salle de Chaillot présente des innovations intéressantes. Outre le traitement des parois et des plafonds témoignant d'une réelle prise en compte des contraintes posées par les salles à grande capacité pour véhiculer la voix et éviter les échos, on trouve, de part et d'autre des gradins et au-dessus du cadre de scène, des chambres de réverbération. Elles doivent leur existence à l'orgue, héritage du premier Trocadéro de 1878, conservé et modernisé. L'orgue exigeant une grande réverbération, ces espaces sont en fait des caisses de résonnance que le chef d'orchestre peut choisir d'ouvrir ou de fermer selon les besoins. Les parois sont couvertes d'un tissu qui masque les volets pivotants, eux-mêmes munis d'éléments absorbants ou réfléchissants. Si aujourd'hui ce type de dispositif qu'on appelle le couplage est exploité dans certaines salles de concert comme la Philharmonie de Paris ou l'auditorium de Lucerne, à l'époque, c'est une option inédite. On doit son existence à l'acousticien Jacques Brillouin, étrangement absent des archives liées à ce projet, mais dont le rôle est attesté par divers documents de presse. Le choix de ce parti pris acoustique est lié à la polyvalence de la nouvelle salle, qui n'est donc pas un théâtre conçu pour la voix parlée, mais bel et bien un auditorium où la vocation musicale étant celle qui génère le plus de contraintes s'impose sur toutes les autres.

Jacques Brillouin s'exprime dans La Revue musicale sur les raisons de ce choix ${ }^{28}$, évoquant comme modèle les vases d'airain décrits par Vitruve dans le livre cinq du De Architectura. L'examen des plans successifs permet de comprendre comment la réalisation des chambres de réverbération s'est concrétisée au fil des étapes du dessin du projet. Carlu prévoyait initialement que l'orgue fût morcelé en trois parties, les grands tuyaux mesurant plus dix mètres de haut se seraient ainsi positionnés de part et d'autre du cadre, tandis que le reste de l'orgue aurait pris place sur la scène. Outre la nécessaire électrification de l'instrument, ce morcellement génère des réserves d'air appelées "local des orgues", précisément là où seront placées les chambres de réverbération quand le choix d'installer l'orgue sur scène et en un seul morceau s'imposera $^{29}$. L'existence de ces chambres de réverbération sur les flancs de la salle, de même que la nature des parois à cet endroit, éclaire également l'interprétation d'une photographie de la salle lors de la session de l'ONU de 1951, conservée dans les archives de Jacques Carlu. On y voit des régies, installées sur les parois latérales et ouvertes sur l'auditorium; il s'agit sans doute des régies de traduction. Leur positionnement correspond très exactement aux chambres de réverbération. La photographie n'est pas très explicite et peut donner des doutes quant au fait qu'il s'agisse bien de la salle de spectacles et non d'un auditorium construit dans le bâtiment éphémère réalisé pour cette session ${ }^{30}$. Toutefois, un article de presse faisant état de cette installation de l'ONU à Chaillot lève le doute, Carlu expliquant que «nul pays - même Genève - n'offrait les possibilités du théâtre de Chaillot dont l'ampleur et les dispositions conviennent particulièrement aux exigences de l'ONU. Grâce à ses parois latérales, à son vaste foyer, l'expérience a prouvé qu'on pouvait en faire une salle d'assemblée absolument unique pour des services complexes et des installations techniques perfectionnées ${ }^{31} »$. Outre ces régies, les photographies de la salle occupée par l'ONU montrent des 
transformations importantes qu'il faudrait encore confirmer par d'autres sources. Concernant l'outil mis à la disposition du TNP un an plus tard, il ne fait aucun doute que la salle avait été restaurée dans son état original. Mais ses premières années d'existence ont peu donné l'occasion de l'utiliser telle qu'elle avait été conçue. Les chambres de réverbération semblent être rapidement tombées en désuétude. À défaut de pouvoir dire si ces chambres ont servi ou non, notons que sur une série de plans exécutés avant la démolition en 1972, on lit très clairement à l'emplacement des chambres de réverbération "dépôt costumes et projecteurs ${ }^{32}$ ». Car, pour être vastes, les locaux du TNP à Chaillot n'en sont pas moins insuffisants à leur exploitation en théâtre régulier de création.

\section{Le TNP de 1952, héritier d'un cahier des charges obsolète}

15 Si l'installation du premier Théâtre national populaire dans la salle des fêtes de 1878 relève d'un imprévu, d'une opportunité immobilière à saisir, en revanche, la marginalité apparente de la place du TNP dans la construction de 1937 est plus étrange. En effet, le concours pour la modernisation de la salle n'oublie pas de reloger le théâtre populaire, mais sans revenir sur son mode de fonctionnement d'alors. Ainsi, une note précise en 1935 au sujet de la salle de spectacles que le théâtre ne devant pas «être exploité quotidiennement, mais seulement trois à quatre fois par semaine, il y aurait lieu de prévoir un personnel réduit au strict minimum et capable, néanmoins, d'assurer la marche de l'établissement ${ }^{33}$ ». À aucun moment il n'a été pensé ou prévu qu'une troupe régulière de création s'installerait dans ces murs. Cet état de fait semble être l'héritage de l'installation du premier TNP au Trocadéro en 1920. On envisageait premièrement d'y programmer les spectacles de la Comédie-Française, de l'Odéon, de l'Opéra et de l'Opéra-Comique, et non d'y créer des spectacles. Une note anonyme, conservée dans les documents relatifs au cahier des charges de ce premier Théâtre national populaire, détaille cette hypothèse :

\footnotetext{
Le Théâtre National Populaire peut être réalisé par l'accord et la collaboration des quatre théâtres subventionnés sur les bases suivantes.

Chacun de ces quatre théâtres subventionnés donne une représentation par semaine. Le programme est constitué par une pièce de son répertoire, ou de toute autre façon, et l'interprétation fournie par sa troupe.

Les représentations ont lieu le mercredi soir, le jeudi en matinée, le samedi soir, le dimanche en matinée.

Un roulement est établi entre les quatre théâtres de manière à leur assurer successivement le profit des représentations les plus avantageuses.

Pour chacune de ces représentations le théâtre organisateur joue à son compte [déduction faite de toutes les taxes et redevances]. Les Théatres Nationaux peuvent, en sus de leur représentation hebdomadaire, obtenir la salle pour d'autres représentations dans les mêmes conditions tarifaires ${ }^{34}$.
}

Le cahier des charges effectif et soumis à Firmin Gémier sera moins étroit quant à la programmation du Théâtre populaire. L'article 9 stipule que les théâtres nationaux "sont autorisés à traiter avec le Directeur du Théâtre Populaire pour réaliser ou faciliter les réalisations de ce théâtre. Le Directeur pourra, en outre, d'accord avec les directeurs des Théâtres Nationaux, choisir dans le répertoire de ces théâtres les ouvrages susceptibles d'être momentanément prêtés $[. . .]^{35} \%$. Ce cahier des charges, décliné en vingt-cinq articles, n'envisage aucunement les activités de création du 
Théâtre populaire, tenu de programmer au minimum cent représentations par an, réparties en œuvres dramatiques et lyriques, auxquelles peuvent s'ajouter ballets, concerts et «films éducatifs ${ }^{36}$ ». Les clauses financières pesant sur le directeur du Théâtre populaire ne manquent pas d'intérêt, mais nous nous limiterons ici à constater que ce directeur d'un théâtre public est en fait bien plus un chef d'entreprise, engagé sur ses fonds personnels, percevant certes une subvention annuelle, mais insuffisante en soi et donc pouvant être complétée de fonds privés par la constitution d'une société. Comme on le voit, ces premiers pas de l'État dans la démocratisation culturelle sont encore très marqués de la culture entrepreneuriale héritée du siècle précédent.

Ce que l'on comprend surtout c'est que le Théâtre populaire est donc perçu comme un lieu de diffusion, pouvant accueillir des spectacles et en donner à l'extérieur. Cependant, ce type d'exploitation pose des problèmes scénographiques et des coûts qui ne sont aucunement pris en compte. Un courrier adressé au ministre par Émile et Vincent Isola, alors propriétaires de nombreuses salles à Paris, dont l'Opéra-Comique qu'ils dirigent, propose, tout en se félicitant de la nomination de Firmin Gémier à la tête de l'Odéon, d'assurer en exclusivité la centaine de représentations attendue au Théâtre populaire, à condition d'en récupérer l'intégralité des recettes, précisément pour compenser les coûts d'adaptation de leurs spectacles à la scène du Trocadéro ${ }^{37}$. On pourrait penser que ces quinze années d'exploitation de la salle des fêtes en théâtre régulier aient permis de tirer des leçons pour le projet de 1937, mais il n'en est rien. La place du Théâtre national populaire dans ce projet de reconstruction n'est pas décisive pour la réalisation architecturale de l'outil mis à sa disposition. Le 15 novembre 1938, un arrêté signé par Jean Zay donne concession à Paul Abram, alors directeur du Théâtre national de l'odéon, pour l'exploitation de la salle de Chaillot. L'outil est neuf, sur le point d'être livré, mais Paul Abram prend immédiatement contact avec les architectes pour définir un programme d'aménagement « indispensable pour l'ouverture à réaliser dans un délai maximum de deux mois ${ }^{38} »$. Ces travaux, approuvés par Carlu, demandent des modifications ou des finitions diverses, des équipements scénographiques au niveau du plateau, des cintres, mais aussi des ajustements plus lourds tels que la modification des casiers à décors, la réalisation de cheminées de contrepoids, ou la diminution de l'avancée du proscenium, cela obligeant à en revoir le gros œuvre et l'habillage côté salle. On sait que, quinze ans plus tard, Jean Vilar demandera au contraire à Camille Demangeat de construire un proscenium pour franchir le cadre de scène. C'est un fait que le concours de 1935 ne prévoyait rien pour la scénographie, l'occasion de donner corps aux expérimentations alors en vogue n'est pas saisie. Pensons par exemple à la scène tripartite conçue par Auguste Perret pour l'exposition des Arts Décoratifs de 1925, ou à d'autres projets non réalisés visant à sortir de l'outil scénique traditionnel ${ }^{39}$. Dès lors, la simplicité de la scène projetée en 1937 sera soulignée par quelques commentateurs et fera écrire à un rapporteur chargé de l'étudier, dans un courrier adressé au président du service des Fêtes et des Spectacles, qu'il a «le regret de [lui] dire que cette scène, telle qu'elle est prévue, aurait pu être

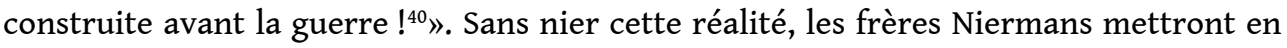
avant des innovations scénographiques certes plus discrètes mais autrement plus durables telles que la fosse d'orchestre recouvrable, le cadre de scène rétractable de dix-neuf à huit mètres d'ouverture, et une paroi permettant de réduire la jauge en coupant la salle sous le balcon; des dispositions là encore inédites et qu'on trouve désormais dans bien des théâtres contemporains. 
me on le voit, les allers-retours entre les documents d'architecture, les commentaires laissés par les usagers ou la presse, recoupés avec une vision plus large des contextes, nous donnent des salles de spectacle du Trocadéro une vision plus riche que les traditionnelles conclusions à l'échec. Le Trocadéro, ce sont aussi les petites salles. La salle Gémier, ouverte en 1967, fut un audacieux théâtre d'essai logé dans l'ancien bar fumoir ${ }^{41}$, mais le premier Trocadéro aussi avait sa petite salle, faisant l'objet d'un cahier des charges et d'une concession spécifique. Il y avait en fait deux salles, situées symétriquement au premier étage des pavillons bordant le corps central, identifiées comme des salles de concerts, l'une d'entre elles deviendra salle de cinéma où l'on fit les premières expériences de cinéma bruité en 1910. Entourée de portesfenêtres, cette salle de 200 places était ironiquement réputée être « la salle de cinéma la plus sûre de Paris $^{42} »$. On en mesure la spatialité quand on se rend aujourd'hui au premier étage du Musée de l'homme. Car ne perdons pas de vue que la première particularité des théâtres qui se sont installés à Chaillot est d'être partie d'un tout, ce vaste palais dont il est impossible d'espérer retracer toute l'histoire et dont Henri Weitzmann nous a laissé un portrait affectueux, celui d'un usager peu soucieux des querelles esthétiques :

Le Trocadéro pour moi, c'est l'enfance, le bonheur. J'étais Alice au pays des merveilles : le Trocadéro était ma maison, les jardins mon jardin et tous les gens qui s'y promenaient, je les croyais invités par mes parents, comme les spectateurs de la grande et la petite salle.

[...] Mes parents étaient concessionnaires du buffet du palais du Trocadéro. Dès l'âge de trois ans on me mettait sur un pouf dans la salle et je fus nourri de tout ce qui se passa dans cette période, des spectacles aux manifestations politiques, Jaurès par exemple que j'entendais sans rien comprendre mais qui prenait aux tripes le petit enfant que j'étais ${ }^{43}$.

De même, les spectateurs du TNP de Vilar quelques années plus tard ne tariront pas d'émotions en dépit des imperfections de l'outil.

\section{NOTES}

1. VILAR (Jean), Mémento du 29 novembre 1952 au $1^{\text {er }}$ septembre 1955, Paris, Gallimard, 1981, p. 111.

2. Dans «Un lieu théâtral : Avignon », paru dans les actes du colloque de Royaumont, Le Lieu théâtral dans la société moderne, Jean Vilar revient sur cette expérience, expliquant tous les problèmes à la fois techniques et symboliques posés par l'utilisation de la cour du Palais des Papes pour le théâtre. (Paris, CNRS, 1963, p. 153-159).

3. Écrire l'Histoire de l'Oral, L'émergence d'une oralité et d'une auralité modernes. Mouvements du phonique dans l'image scénique (1950-2000), programme financé par l'Agence nationale de la recherche, sous la direction de Marie-Madeleine Mervant-Roux.

4. GIEDION (Siefried), Espaces, temps, architecture, Paris, Denoël-Gontier, 1978 [1968], p. 46.

5. DALY (César), «Les deux palais de l'exposition considérés dans leur rapport avec l'art », dans Revue générale d'architecture et des travaux publics, n XXXIV, 1878, p. 1999.

6. Cette citation d'Huysmans est consignée dans un article intitulé «Le Trocadéro de 1878 ", conservé dans le fonds Carlu (Institut français d'architecture, A 010 204/3). 
7. Note manuscrite anonyme traduisant un extrait d'un article de la Neue Freie Presse, «La Presse française et la presse étrangère » (Arch. nat., F/12/3265).

8. Journal de la Vienne à Poitiers, 22 avril 1877. « La Presse française et la presse étrangère » (Arch. nat., F/12/3265).

9. Seize millions de visiteurs en 1878 pour quinze millions en 1867.

10. VIEL (Edmond), Projet pour un opéra populaire, Paris, E. Dentu, 1870, p. 3.

11. Les différentes esquisses de l'Orphéon sont conservées dans le fonds Gabriel Davioud de la Bibliothèque de l'Hôtel de Ville à Paris, de même que les plans du Palais du Trocadéro.

12. GOSSET (Alphonse), Traité de la construction des théâtres, Paris, Baudry et $C^{\mathrm{ie}}, 1886$, p. 51.

13. Ibid.

14. LACHÈz (Théodore), Acoustique et optique des salles de réunion publique, Paris, imprimé par l'auteur, 1848.

15. GOSSET (Alphonse), op. cit, p. 51.

16. Aménagement de la salle du Trocadéro en vue d'y donner des concerts populaires, Séance au Sénat du 26 décembre 1903 (Arch. nat., F/21/6145).

17. De nombreux détails techniques sont donnés dans l'ouvrage de l'exposition universelle, Le Palais du Trocadéro, le coteau de Chaillot, le nouveau palais, les dix-huit mois de travaux, renseignements techniques, Paris, A. Morel et $\mathrm{C}^{\mathrm{ie}}, 1878$.

18. Le dossier concernant les appareils de chauffage et ventilation est conservé aux Archives nationales sous la cote $\mathrm{F} / 12 / 347$.

19. Ces éléments sont conservés aux Archives nationales sous la cote $F / 21 / 6145$. Voir également Dubouily (Sandrine), "Chaillot 1937. Ambitions et réalités d'une recherche architecturale et acoustique » dans MERVANT-Roux (Marie-Madeleine), Bovet (Jeanne) [dir.], L'Echo du théâtre 2 La Scène parle, Voix, acoustiques et auralités (seconde moitié du $\mathrm{XX}^{e}$ siècle), Revue Sciences/Lettres [En ligne], 6 | 2019, mis en ligne le 10 décembre 2018. URL : http://journals.openedition.org/rsl/1986 20. GÉMIER (Firmin), M. Gémier et le théâtre populaire, exposé adressé à M. Paul Léon, directeur des Beaux-Arts, 24 décembre 1921, p. 1 (Arch. nat., F/21/5269).

21. Firmin Gémier, Compte-rendu de l'année 1929, p. 9 (Arch. nat., F/21/3984).

22. Théâtre national populaire, Compte-rendu de l'année 1930, p. 10 (Arch. nat., F/21/3984).

23. Théâtre national populaire, Compte-rendu de l'année 1922, p. 21 (Arch. nat., F/21/3984).

24. Les éléments relatifs à cette polémique sont consultables aux Archives nationales sous la cote 19960511/3. Plusieurs personnalités sont ainsi signataires d'une pétition contre la reconstruction du Trocadéro. La revue L'Architecture d'aujourd'hui est également désignée responsable en partie de cette opposition au projet de Carlu, Boileau et Azéma.

25. Ce recensement est conservé à l'Institut français d'architecture sous la cote CARJA-D-35-3 IFA $203 / 3$.

26. Citons par exemple GournAY (Isabelle), Le Nouveau Trocadéro, Liège, IFA-Mardaga, 1985 ou ORY (Pascal), Le Palais de Chaillot, Arles, Actes Sud, 2006.

27. Arch. nat., 19960511/1.

28. BRILLOUIN (Jacques), «La salle de spectacle du nouveau Trocadéro », dans La Revue musicale, juin-juillet 1937. Dossier « L'exposition et la musique ».

29. Les documents relatifs aux transformations de l'orgue sont conservés sous la cote $19960511 / 3$.

30. Un bâtiment éphémère en $U$ vient en effet se greffer au Trocadéro perpendiculairement à celui-ci côté jardin. 500 bureaux y sont aménagés.

31. «Un palais éphémère se greffe au palais de Chaillot sans rien coûter au budget de la France ", dans Le Figaro littéraire, 12 mars 1951.

32. «Relevé des locaux annexes» (Arch. nat., 19900195/104). 
33. Document conservé dans une pochette intitulée "Théâtre du Trocadéro » (Arch. nat., F/ 12/12150).

34. Document anonyme conservé dans la pochette "Trocadéro, cahier des charges du Théâtre Populaire» (Arch. nat., F/21/6148).

35. Ministère de l'Instruction publique et des Beaux-Arts, Cahier des charges du Théâtre populaire, 14 mars 1922, p. 4 (Arch. nat., F/21/6148).

36. Ibid., p. 3.

37. Courrier du 2 décembre 1921, conservé dans la pochette «Trocadéro. Cahier des charges du Théâtre Populaire » (Arch. nat., F/21/6148).

38. Annotation manuscrite de Paul Abram sur le texte dactylographié « Salle de théâtre du Palais de Chaillot. Programme des travaux d'aménagement établi en accord avec M. Paul Abram ",

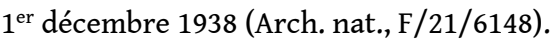

39. On pourra citer ici par exemple un étonnant projet breveté de Paul Castan et Roger Souchère, conçu précisément pour cette exposition universelle de 1937 et proposant une scène annulaire avec un cylindre portant parterre et balcons, pivotant au centre de celle-ci. Combiné à une scène traditionnelle mais aussi des cintres et tournettes disposés sur toute la surface de l'anneau de scène, ce projet était supposé réaliser 38 tableaux minimum par spectacles. CASTAN (Paul) et SOUCHère (Roger), Le Théâtre à salle tournante, 1933, Société d'Impression industrielle, $11 \mathrm{p}$. 40. Anonyme, Courrier conservé dans le dossier "4-Salle de théâtre, correspondances etc.", datant probablement de 1935 (Arch. nat., F/12/12184).

41. Voir DUPUY-OLIVIER (Anaïs), «Le scénographe Jacques Le Marquet et le Palais de Chaillot", publié dans ce volume.

42. "Le Trocadéro. Note d'écoute d'Henri Weitzmann», document dactylographié anonyme conservé dans les archives de Jacques Carlu (CARJA-D-35-3. IFA 203/3).

43. Ibidem.

\section{RÉSUMÉS}

En visite à Krefeld, Jean Vilar admire les vastes espaces de ce théâtre fraichement reconstruit : "Le jour inonde les couloirs, les loges, les bureaux et le sous-marinier que je suis (U-boat "Chaillot" immobilisé par 25 mètres de fond) se promène avec délice dans cette lumière ". Lorsque le TNP s'installe au Trocadéro en 1953, la salle inaugurée en 1938 a déjà connu d'importantes transformations, certes provisoires, pour accueillir l'ONU. Jean Vilar fera réaliser des travaux immédiats pour adapter la salle de spectacle au théâtre. Dès lors, le Palais de Chaillot abrite une activité pour laquelle il n'était pas prévu, celle d'un lieu de création pour une troupe permanente. Un changement d'usage que l'activité du TNP, pourtant installé au Trocadéro depuis 1920, n'avait pas anticipé. En recoupant les archives de la commande, des architectes et des divers commentateurs, nous nous efforcerons de comprendre les raisons qui ont prévalu à la réalisation de cette grande salle de 2800 places, sous la dalle du Trocadéro, ses innovations mais aussi ses limites pour une exploitation régulière comme théâtre. 
INDEX

Mots-clés : Théâtre national populaire, Trocadéro, Palais de Chaillot, Vilar (Jean), Davioud (Gabriel), Bourdais (Jules), Gémier (Firmin), Niermans (Édouard), Niermans (Jean), exposition universelle 1937, ONU

\section{AUTEUR}

\section{SANDRINE DUBOUILH}

Architecte DPLG, professeure des universités EA CLARE 4593 - Université Bordeaux Montaigne, membre du projet ANR ECHO 


\title{
Le scénographe Jacques Le Marquet et le Palais de Chaillot
}

\author{
Anaïs Dupuy-Olivier
}

1 Jacques Le Marquet (1927-2017) a travaillé vingt ans au sein du Palais de Chaillot en tant que membre du Théâtre national populaire (TNP). Entré au TNP en 1952 comme second adjoint constructeur, il succède à Camille Demangeat à la tête de la régie construction en 1954 et assure cette fonction jusqu'à son départ en octobre 1972. De par cette fonction, il a réuni une documentation importante sur le TNP et le Palais de Chaillot de cette période, complémentaire des autres sources d'archives existantes à la Bibliothèque nationale de France, aux Archives nationales et aux Archives départementales des Hauts-de-Seine. En effet, au moment du départ de Jean Vilar de la direction du TNP, puis de celui de Georges Wilson, J. Le Marquet a dû classer les archives de la régie construction en vue de leur versement aux Archives nationales. Lors de ce tri, il a gardé certains documents soit parce qu'ils lui appartenaient (journaux de bord, correspondances, photographies), soit parce que c'étaient des doubles des archives remises (programmes, plans de théâtre), soit enfin parce que « le document s'était égaré là »" Ces archives sur le TNP, conservées au département des Arts du spectacle de la BnF, constituent 165 pochettes classées par ordre chronologique des spectacles montés et des tournées.

2 En croisant ces différentes sources d'archives², il nous a été possible d'appréhender la façon dont le TNP a composé avec les espaces du Palais de Chaillot et la façon dont un de ses membres a travaillé dans et avec ces espaces. Au travers de l'exemple de J. Le Marquet, nous souhaiterions rendre compte de ces recherches sur les espaces de travail et le fonctionnement du TNP. Notre article s'articulera autour de deux axes: nous étudierons d'abord l'activité de régisseur de J. Le Marquet, puis son rôle dans les évolutions architecturales des espaces occupés par le TNP.

\section{Jacques Le Marquet, régisseur au TNP}

Après une formation au dessin et à la sculpture, Jacques Le Marquet aurait souhaité devenir sculpteur, mais il comprend rapidement qu'il ne pourra pas en vivre C'est ainsi 
qu'en 1951, il entre comme assistant technique dans des théâtres parisiens. En février 1952, il est engagé au TNP comme " petit régisseur $»^{3}$, selon les mots de Jean Vilar.

4 Le TNP comprend trois entités, un service administratif, une troupe et un service technique. Ce dernier s'organise en régies prenant chacune en charge un aspect du spectacle. Sous la régie générale, travaillent trois régies techniques, la régie construction, la régie des éclairages et la régie du son. Elles ont à leur tête un régisseur et plusieurs techniciens en font partie. En 1952, c'est Camille Demangeat ${ }^{4}$, ancien chefconstructeur de Louis Jouvet, qui dirige la régie construction. J. Le Marquet, second adjoint constructeur, se forme à son contact. Lorsque deux ans plus tard Camille Demangeat quitte le TNP, Jean Vilar nomme J. Le Marquet pour le remplacer.

5 À ce titre, Le Marquet est l'intermédiaire entre le scénographe, concepteur de décors, et les équipes chargées de réaliser décors et accessoires (constructeurs, peintres, accessoiristes, tapissiers, serruriers). Il est amené à dresser des plans, à surveiller la construction des décors et leur installation sur scène. Il est également chargé d'organiser les tournées et le Festival d'Avignon pour ce qui incombe à son service : construction des décors et du matériel nécessaire à la modification ou à la construction de scènes, transport, montage.

6 Un atelier est placé sous la tutelle de la régie construction. Il est divisé en plusieurs services, spécialisés chacun dans un corps de métier :

- le service de construction à proprement parler réalise les structures porteuses des scènes et des décors (châssis, fermes ${ }^{5}$ ), les praticables ${ }^{6}$ et le gros mobilier ;

- le service des accessoires construit, loue ou achète les objets de plus petites dimensions ; les décorateurs lui fournissent des dessins et des plans pour leur réalisation ;

- le service de tapisserie s'occupe des rideaux et de tous les revêtements scéniques (sols, murs et mobilier). 


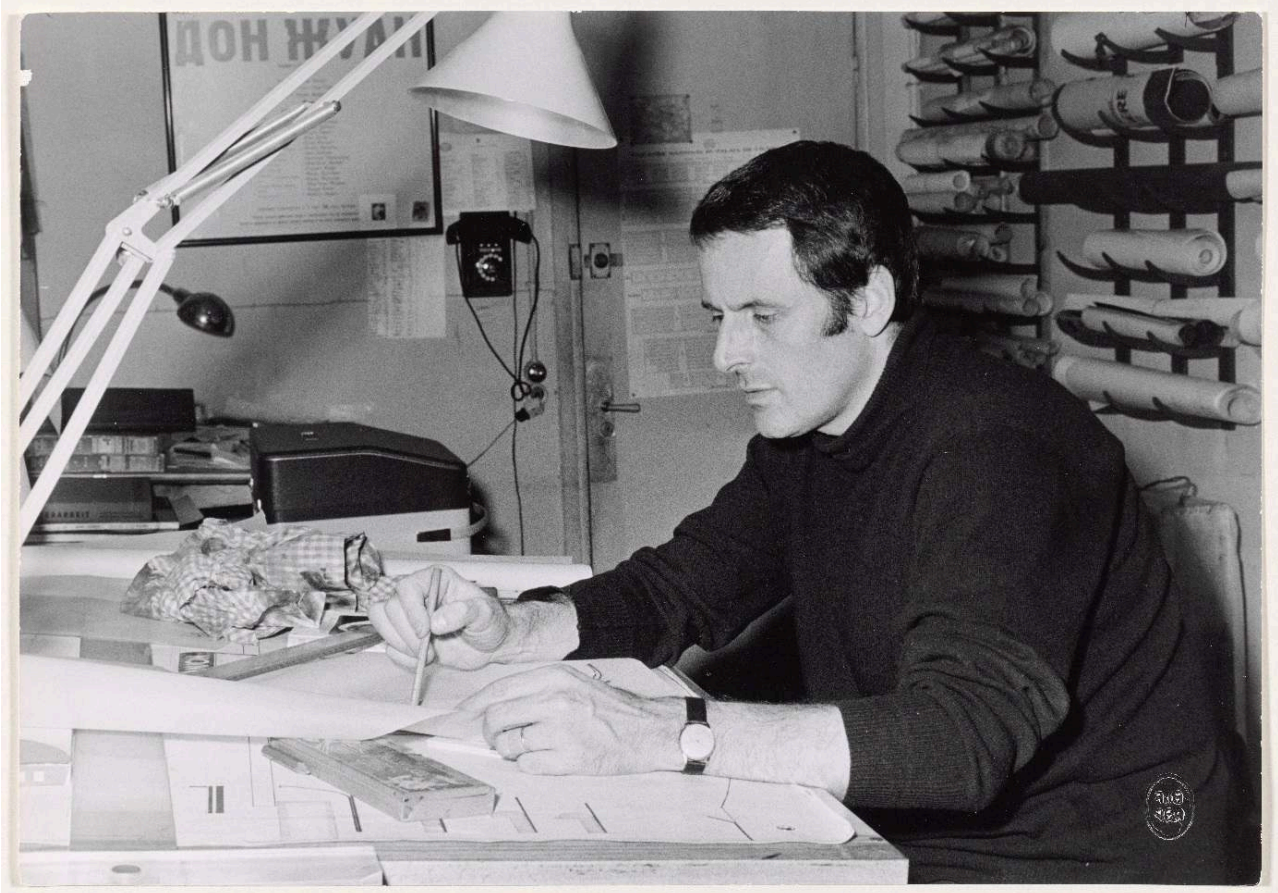

BnF, ASP, 4-COL-COL-53 (381), Jacques Le Marquet devant son bureau à Chaillot.

(photographie de Roger Pic)

7 Ces services, ainsi que les bureaux du personnel, sont installés en sous-sol, autour ou à proximité de la grande salle. Ainsi, le bureau de J. Le Marquet et de son adjoint André Bataille se trouve au deuxième niveau du sous-sol, sur le côté cour de la scène ce qui correspond à l'aile du palais orientée vers Paris ${ }^{7}$; au même niveau, en passant de l'autre côté de la scène, côté jardin, on accède au bureau de la régisseuse des costumes, Huguette Gresse, et à l'atelier de retouches des costumes. L'atelier des accessoiristes et le foyer des machinistes sont installés au niveau de la scène, le premier côté jardin, le second côté cour ${ }^{8}$. L'atelier de menuiserie quant à lui est plus éloigné de la salle, installé au niveau du balcon de la grande salle, sur le palier à proximité de l'aile Passy. Après la construction de la salle Gémier qui prend la place du bar-fumoir, ce dernier est installé à l'emplacement de l'atelier de menuiserie, élargi de la sortie de secours. Les membres $\mathrm{du}$ TNP se demandent alors où déplacer leur atelier. Les archives de Le Marquet nous apprennent que deux emplacements ont été envisagés: un plan de 1965 montre l'atelier à côté de la salle Gémier, le long des jardins ; un plan de 1966 propose un avantprojet d'atelier de menuiserie du côté de l'aile Passy. Nous pensons que l'atelier n'a pas été déplacé car, dans une note rétrospective, Le Marquet indique qu'à partir des années 1960, il fait appel au constructeur Claude Jouen et à sa société « Les artisans et ouvriers réunis » (A.O.R.) pour la construction des décors9.

Le TNP ne dispose pas d'atelier de peinture dans le Palais de Chaillot. La régie construction travaille jusqu'en 1972 avec le peintre de décor Raymond Jousselin. Ce peintre indépendant possède un atelier, d'abord installé rue du Moulin Joli, puis rue des Pyrénées dans le $\mathrm{xx}^{\mathrm{e}}$ arrondissement. Lorsqu'il travaille pour le TNP, il vient la plupart du temps peindre à Chaillot, notamment pour les éléments de décors de taille moyenne. Il s'installe « un peu partout dans Chaillot $»^{10}$, et en particulier dans le grand escalier 
condamné côté Passy débouchant sur le péristyle du musée de la Marine dont il recouvre le marbre avec de vieilles affiches pour ne pas le salir ${ }^{11}$.

9 De manière générale, le TNP manque d'espaces adaptés pour fonctionner de manière optimale : il n'y pas de salle de réunion ou de lecture pour plus de cinq personnes, il existe une seule salle de répétitions " mal chauffée et mal aérée ${ }^{12}$, il manque des loges pour les comédiens, il n'y a pas de magasins pour stocker décors, accessoires et costumes. Ces derniers sont entreposés dans les couloirs, les escaliers, les accès, les dégagements du palais, contrevenant aux règles de sécurité et se détériorant. Ainsi, lors de l'inventaire du matériel scénique en 1971, sur le palier Passy étaient entreposés un certain nombre de décors : ceux de Luther, Hamlet, du Roi Lear, de Chêne et Lapins angora, du Diable et le Bon Dieu ou encore de La Danse de Mort ${ }^{13}$. Notons qu'à cette époque, le TNP dispose également d'un magasin en banlieue, à Herblay, où sont stockés les décors des Enfants du soleil, de Romulus le Grand et de Maître Puntila et son valet Matti ${ }^{14}$.

10 Le travail de régisseur de J. Le Marquet nécessite donc de s'adapter aux espaces du Palais de Chaillot qui, malgré sa grande superficie, n'est pas fait pour accueillir les activités techniques d'un théâtre. C'est dans ce sens qu'en mars 1961, il conçoit ce qu'il appelle un «projet utopique de ce que serait une grande salle moderne correspondant aux besoins du TNP $»^{15}$. Comme il le précise dans une note plus tardive, «il ne s'agit pas de la salle elle-même, mais de tout ce qui la sert, autour ${ }^{16}$. Le Marquet remet onze plans à l'administrateur du TNP, représentant les différents services utiles à la bonne marche d'un théâtre, tant dans ses aspects administratifs que techniques et artistiques : le « bloc » administratif (direction, secrétariat, comptabilité) ; le « bloc » du personnel de la salle; le «bloc» du personnel du plateau; le «bloc» des régies éclairages, son et cinéma ; le «bloc » construction et magasins; le «bloc» costumes et perruques ; le foyer technique ; les loges pour les comédiens; la cantine ; les sanitaires et les vestiaires.

11 J. Le Marquet pense à tout et notamment aux espaces qui font défaut à son travail quotidien et à celui de ses collègues. Il propose, pour le "bloc » construction et magasins, un espace de $2230 \mathrm{~m}^{2}$ composé de sept ateliers, de deux magasins de décors, d'un magasin pour les costumes et d'un magasin pour les accessoires, meubles et matériel électrique. Ces locaux, installés dans les sous-sols, seraient complétés, dans les étages, près des loges des comédiens, par un espace dédié à la confection des costumes et des perruques. Ces espaces de travail possèdent toutes les structures nécessaires à l'élaboration et à la conservation des décors et des costumes.

12 Ce projet n'aura pas de suite vraisemblablement car trop ambitieux et impossible en l'état du Palais de Chaillot et des moyens dont dispose le théâtre pour fonctionner. Malgré tout, cette réflexion sur les espaces du Palais de Chaillot ainsi que les compétences développées par J. Le Marquet dans le domaine de la scénographie d'équipement, l'amènent tout naturellement à participer aux projets de réaménagement et de construction relatifs aux espaces occupés par le TNP.

\section{La salle Gémier (1963-1967)}

13 Le premier projet qui va mobiliser Jacques Le Marquet est celui de la construction d'une "petite salle» ou "petit théâtre " en complément de la salle existante qui se 
caractérise par ses vastes dimensions tant en termes de surfaces et de distances que de jauge.

\section{Les prémices du projet}

14 Il faut remonter en 1951. Si Jean Vilar a été nommé à la tête du Théâtre national populaire sis dans le Palais de Chaillot, c'est du fait de deux expériences: sa participation aux mouvements de démocratisation et de décentralisation culturelles après la guerre l'amenant à créer un festival de théâtre à Avignon, et « son aisance à maîtriser l'espace de la cour d'honneur du Palais des Papes ${ }^{17}$ dont les dimensions sont similaires à celles de la salle de Chaillot. Mais, bien que capable d'adapter son répertoire, le jeu de ses acteurs, ses décors et ses costumes à ces vastes dimensions, le TNP perçoit rapidement les contraintes qu'elles imposent. Les tournées en banlieue parisienne, en province ou à l'étranger confortent Vilar dans l'idée que le théâtre se joue le mieux dans un cadre de jeu plus restreint, d'environ 12 mètres de large sur 6 à 7 mètres de hauteur par 6 mètres de profondeur. C'est dans cette perspective et dans celle de renouveler le répertoire du TNP qu'en 1959, Vilar obtient d'André Malraux, ministre d'État chargé des Affaires culturelles, la concession du théâtre Récamier pour y présenter les œuvres d'auteurs contemporains. Dans ce théâtre d'essai, Vilar monte des pièces d'Armand Gatti, Boris Vian, Samuel Beckett, Roger Pinget et René de Obaldia, mais aussi d'auteurs moins contemporains comme Brecht ou Ibsen. Ce théâtre ne dure que deux saisons et s'arrête en 1961 faute de fréquentation et de rentabilité financière. 18

\section{Le projet de salle provisoire, 1963-1964}

15 En février 1963, avant même le départ de Jean Vilar et la nomination officielle de Georges Wilson à la tête du TNP (7 juillet 1963), des discussions s'engagent au sein de l'équipe de direction, "une recherche est lancée, des besoins sont formulés »" J. Le Marquet les traduit en croquis : le 20 mai, il remet à l'administrateur du TNP, Lucien Fresnac, un premier ensemble de plans et de notes étudiant l'aménagement d'un petit théâtre. L. Fresnac lui répond favorablement le 27 mai, jugeant qu'après quelques modifications, «ce dossier doit être remis aux Autorités de Tutelle $»^{20}$. Entretemps, Georges Wilson prend contact avec Jean de Mailly, architecte en chef, conservateur du Palais de Chaillot, pour lui exposer leur projet. Le 29 mai 1963, a lieu une première réunion rassemblant, du côté du TNP, Wilson, Le Marquet, le régisseur des éclairages Pierre Saveron et Pierre Sonrel ${ }^{21}$, du côté des "autorités de tutelle ", Émile Biasini, directeur du Théâtre, de la Musique et de l'Action culturelle au ministère des Affaires culturelles, Jean Mailly et son second, Louis Blanchet. Premier contact "glacial, désagréable ${ }^{22}$ aux dires de Le Marquet. Les membres du TNP ont l'impression de ne pas être compris. Leur projet serait, dans un premier temps, de construire une petite salle provisoire dans le bar-fumoir (aile Paris) qui pourrait être réalisée rapidement (d'ici janvier 1964) dans l'attente d'une salle définitive sous les emmarchements de l'aile Passy, qui ouvrirait deux ans plus tard; le bar serait temporairement installé ailleurs, peut-être à l'extrémité du grand foyer. 


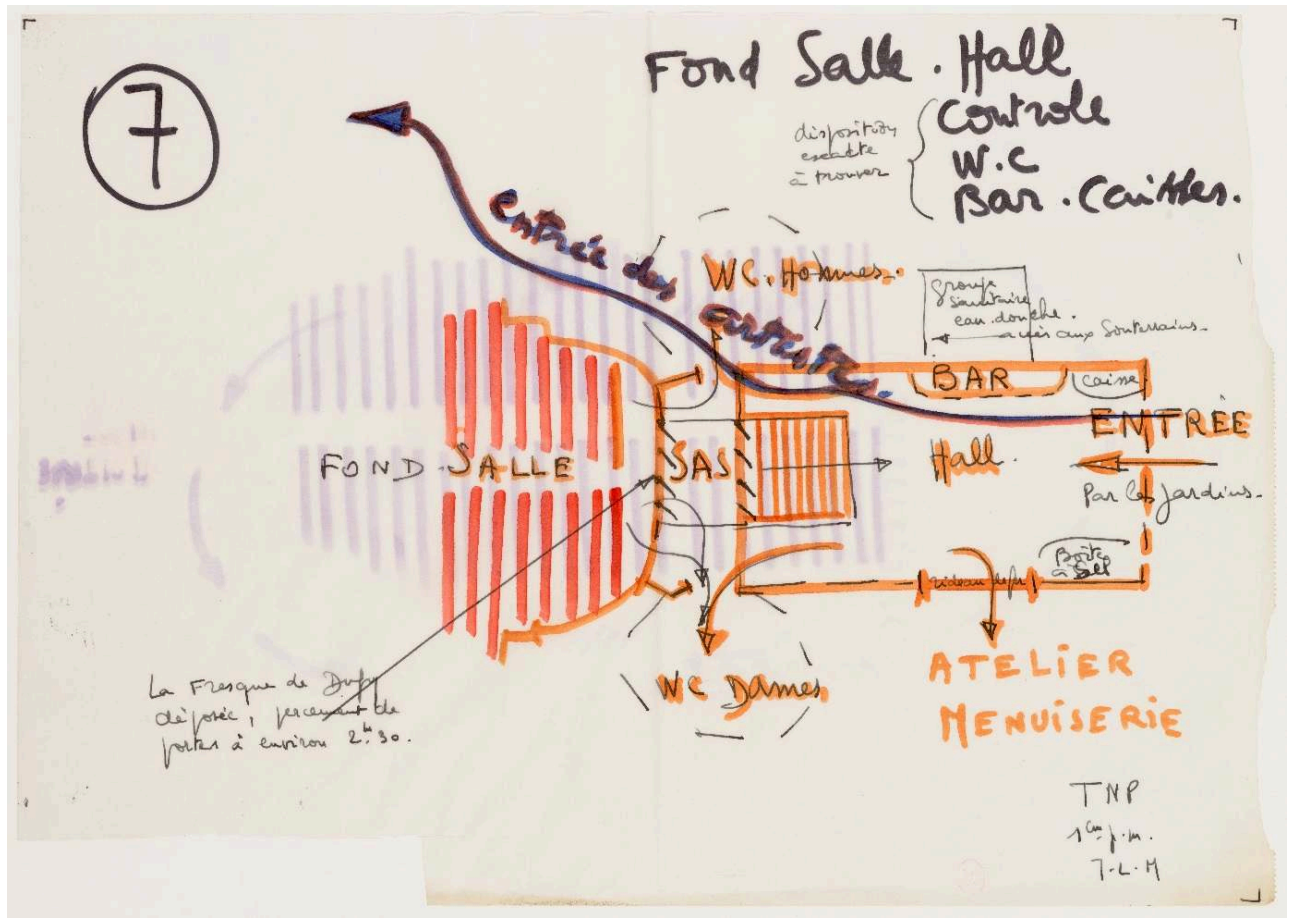

BnF, ASP, 4-COL-53 $(134,4)$, plan sur calque de Jacques Le Marquet pour la petite salle provisoire, 29 mai 1963

16 Ils souhaitent une salle " dont le ton soit l'essai, mais pas seulement l'essai, et [leur] expérience commune de dix ans un peu partout [leur] a prouvé qu'un théâtre trop typé devient inutilisable ${ }^{23}$. Ils veulent « qu'on y puisse recevoir des troupes n'ayant pas les mêmes conceptions, qu'elles soient classiques ou d'extrême avant-garde. Pour cela, il faut que cet instrument, si modeste soit-il, contente "presque" tout le monde, car le T.N.P. lui-même, en définitive, n'y montera pas uniquement des pièces d'avant-garde ; le penserait-on au départ, que cela se démentirait ensuite ${ }^{24}$.

«Va alors s'engager une négociation difficile dont [J. Le Marquet sera] le scribe. [...] Et pour beaucoup l'auteur... $»^{25}$ Les réunions, régulières jusqu'à l'automne 1963, se raréfient en 1964. On hésite longtemps sur l'emplacement de la salle, sur son caractère provisoire ou définitif. Dans l'année 1964, le caractère définitif de la salle et son emplacement au niveau du bar-fumoir sont actés sans que l'on puisse dater précisément ces décisions. Le Marquet continue à fournir plans, notes et programme d'équipement technique à Jean de Mailly qui les annote et les transmet aux entreprises.

\section{La construction de la salle, 1965-1966}

Au sein des archives consultées, on voit apparaître les premiers plans de Jean de Mailly et des entreprises travaillant pour lui, en 1965. Ces plans permettent d'appréhender les différentes composantes de la salle: dessous, combles, ventilation, implantations lumineuses, escalier de service, détails du garde-corps du balcon... La construction débute dans le courant de l'année. Les fresques de Raoul Dufy et Othon Friesz sont déposées, et après restauration, vont l'une dans un musée, l'autre dans une maison de la culture ${ }^{26}$. Le plafond est élevé de 2 mètres. On creuse pour installer la machinerie du 
plateau tournant et la fosse d'orchestre, pour surbaisser les premiers rangs de fauteuils et disposer les autres en gradins.

Les équipes du TNP, et au premier chef l'administrateur du TNP Jean Ruaud ${ }^{27}$ et Le Marquet, suivent de près l'avancée de la construction : précisions sur le matériel d'équipement ; références des cycloramas ${ }^{28}$ et des fonds de scène ; émission de réserves (insonorisation insuffisante entre la salle et ses alentours) et de recommandations (assurer l'étanchéité à la lumière de la porte d'entrée; installer des lignes téléphoniques entre la régie générale, le contrôle et le service habillement) ${ }^{29}$. Ils sont conviés aux réunions avec les architectes.

La pré-réception des travaux a lieu le 29 septembre 1966, la réception provisoire le 17 octobre, une note de service du 24 novembre annonce au personnel le nom de la salle : «T.N.P. Petite salle (salle Gémier) ». Jean Ruaud ajoute : «Par la suite, quand tous se seront habitués, elle prendra le nom de "Salle Gémier" ${ }^{30}$.

21 Bien que quelques aménagements prévus ne soient pas encore achevés (panneau d'affichage extérieur, local destiné à la direction, local pour les machinistes et les accessoiristes dans le souterrain d'accès, manœuvre à la main du rideau d'avant-scène, rehaussement des cinq premiers rangs de fauteuils) ${ }^{31}$, la salle ouvre le 19 janvier 1967 avec deux pièces en un acte, Les ancêtres redoublent de férocité de Kateb Yacine et La grande imprécation devant les murs de la ville de Tankred Dorst. La réception définitive de la salle aura lieu le 7 février 1968 sous la présidence du Contrôleur général des travaux des bâtiments civils et des palais nationaux.

\section{Caractéristiques de la salle}

La salle et ses annexes sont construites dans l'emprise de l'ancien bar-fumoir conçu par Jacques Carlu, c'est-à-dire dans un espace limité et contraint qui empêche toute extension ainsi que la construction de certains équipements scéniques ${ }^{32}$. Comme le dit Jean de Mailly dans une interview en 1967, « l'architecte devait [...] d'abord "inventer" un espace possible dans la surface attribuée au T.N.P. [...] Dans l'impossibilité de caréner les équipements techniques, il fut décidé de les montrer et de tirer parti de cette suggestion, en affirmant l'esprit "théâtre d'essai" et en traitant l'ensemble en "laboratoire" comme le souhaitaient les scénographes et les régisseurs [...] $»^{33}$.

D'une surface de $980 \mathrm{~m}^{2}{ }^{34}$, la salle est un ovoïde de 22 mètres de large et de 30 mètres de long organisé sur trois niveaux: un orchestre, un balcon avec cabines techniques derrière, des locaux techniques. Elle peut accueillir 531 spectateurs (402 à l'orchestre et 129 au balcon). Les fauteuils sont noirs, les passerelles noires ou gris métallique, les tapis et les murs couleur tabac. Les poteaux du bar sont gardés mais habillés autrement. Cette salle ramassée et d'aspect austère a pourtant toutes les caractéristiques d'une salle moderne et fonctionnelle.

La scène ne dispose pas de proscenium ${ }^{35}$. Elle comprend une fosse d'orchestre pouvant accueillir six musiciens. Le cadre de scène, d'une hauteur maximale de 5,80 mètres et d'une largeur maximale de 10,60 mètres, peut être obturé par un diaphragme métallique noir, glissant sur les murs de la salle, tel celui des appareils photographiques: "Vous imaginez l'effet, avec ce diaphragme laissant apparaître d'abord un personnage seul puis, organisé autour de lui, tout un décor $»^{36}$. 
Figure 3

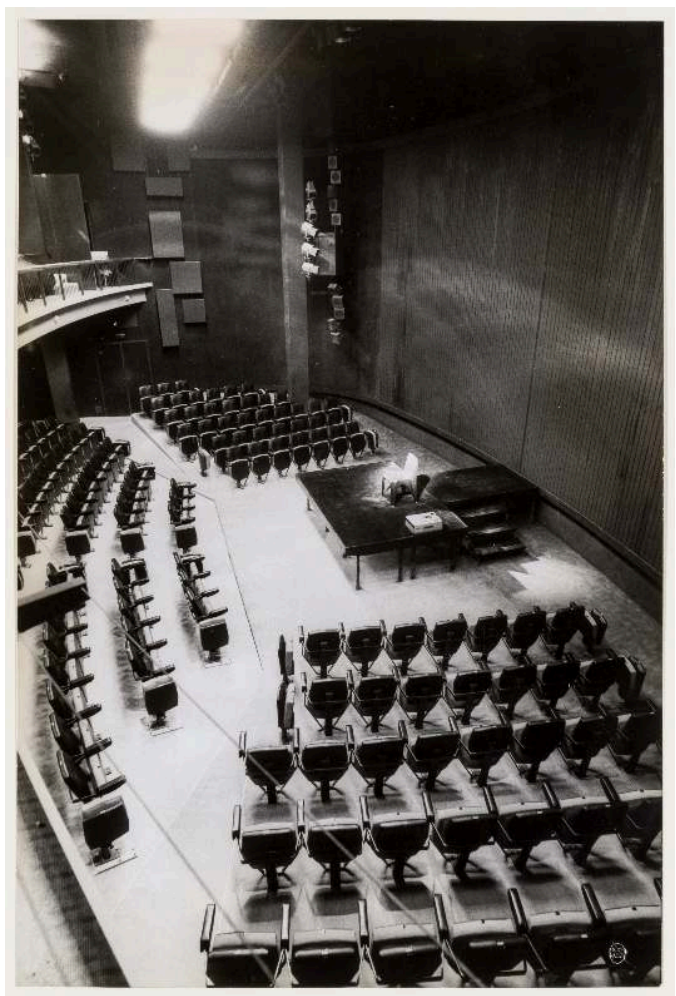

BnF, ASP, FOL-COL-53 (44, 4), dispositif de Jacques Le Marquet pour L'Amante anglaise, 1968 (photographie de Roger Pic)

26 La scène est équipée d'un rideau d'avant-scène, d'un rideau de fer, de trois cycloramas au lointain ${ }^{37}$ (noir, gris, bleu-ciel). Elle n'a ni rampes d'éclairage ${ }^{38}$, ni cintres ${ }^{39}$, mais un gril ${ }^{40}$ d'une hauteur de 8 mètres. La scène est tournante sur 8 mètres de diamètre et comprend une trappe d'apparition. On communique de cour à jardin par un passage sous la scène, l'accès du matériel scénique se fait par le fond de la salle.

Le système d'éclairage et l'installation électro-acoustique sont très perfectionnés. Des projecteurs et des haut-parleurs sont répartis sur plusieurs passerelles dans la salle et sur scène. L'installation sonore offre des possibilités d'enregistrement et de mixage tout en permettant la diffusion d'effets sonores dans la salle et sur scène. La salle dispose également d'un équipement pour projections cinématographiques. Tous les appareillages techniques sont apparents.

Enfin, autour de la salle, sont installés neuf loges pouvant accueillir vingt comédiens, ainsi qu'une loge pour le personnel.

Les régisseurs de la grande salle - Pierre Saveron pour l'éclairage et M. Bérard pour le son - ainsi que les chefs machiniste et accessoiriste, Pierre Musette et Jean-Claude Lecourt, assurent le suivi technique et logistique de la petite salle. Ils sont secondés par des équipes d'électriciens, de machinistes et d'accessoiristes. Quant au personnel d'habillement, il se compose de deux habilleuses encadrées par la régisseuse des costumes Huguette Gresse.

Aucune communication n'est prévue entre les deux salles du Palais de Chaillot. Les issues de secours de la petite salle donnent dans le grand foyer. 
31 L'accès principal à la salle Gémier se fait par les jardins, mais on peut également y accéder par la place du Trocadéro. Le couloir de l'accès principal est bordé de part et d'autre par les caisses, les vestiaires, des sanitaires et un bar.

On se rappelle que la construction de la salle Gémier à l'emplacement du bar-fumoir a pour incidence le déplacement de celui-ci sur le palier opposé, le palier Passy. Le nouveau bar qui ouvre le 12 novembre 1965 est plus spacieux que le précédent : $500 \mathrm{~m}^{2}$, 70 tables, 280 sièges.

\section{Bilan sur les premières années de fonctionnement de la salle Gémier}

33 Jusqu'en 1972, la programmation de la salle Gémier est fidèle à ce que Georges Wilson déclare au journal Bref au moment de son ouverture : «[...] Je ne veux pas en faire un théâtre de répertoire, je monterai des pièces d'auteurs inconnus, des spectacles expérimentaux, je ferai appel à de jeunes metteurs en scène. Sans me préoccuper de la sécurité. Le T.N.P. doit devenir aussi un centre de recherches et ne pas se figer. Sinon, ça ne vaut pas la peine $\star^{41}$. On y joue des auteurs comme Fernando Arrabal, Edward Bond, Tankred Dorst, Marguerite Duras, Jean Vauthier, Martin Walser ou encore Kateb Yacine, montés par des metteurs en scène de la nouvelle génération, Patrice Chéreau, Jorge Lavelli, Claude Régy, Jacques Rosner, Jean-Marie Serreau et bien d'autres.

34 Les retours du public sont positifs, tant sur la salle que sur sa programmation : "Une surprise agréable. Très belle en sa sobriété sévère: couleurs sombres qui donnent l'impression de profondeur, de salle obscure qui invite à la concentration de l'attention, fauteuils très confortables; bonne pente de la salle, bonne disposition des fauteuils pour voir de loin, heureuses proportions de la salle. Excellente acoustique $! \gg^{42}$ D'autres spectateurs soulignent "la distinction $»^{43}$ et le confort de la salle, ainsi que les proportions de la scène, "assez grande et vaste pour une salle à petites dimensions ${ }^{44}$. Les spectateurs reconnaissent que cette salle est davantage appropriée au théâtre expérimental, à la "présentation de pièces "d'avant-garde" ou tout simplement nouvelles, inconnues du grand public $»^{45}$. Ils sentent que «dans ce lieu quelque chose peut naître $»^{46}$ et qu'en offrant des « spectacles d'un style différent, [cette salle] ne peut qu'enrichir l'expérience fructueuse du T.N.P. $»^{47}$.

35 J. Le Marquet aura donc eu un rôle important dans la conception de la salle Gémier, en ayant «fourni [...] l'essentiel des données spatiales et techniques, mais aussi l'emplacement possible d'un théâtre de 550 places dans Chaillot même. [Il a] veillé à tout. Pas à pas, [il a] corrigé toutes les générations de plans de de Mailly $»^{48}$. Précisons en outre qu'il n'a pas été rétribué pour ce travail « s'estimant suffisamment payé pour ce que [il] faisait par ailleurs (direction technique) et notamment par ce [lui] rapportaient les scénographies au T.N.P. $»^{49}$.

\section{Les projets de refonte de la grande salle (1963-1972)}

36 Lors de la construction du Palais de Chaillot ${ }^{50}$, sa salle a été conçue comme un auditorium, convenant bien aux manifestations musicales, chorégraphiques et 
cinématographiques, mais beaucoup moins aux représentations théâtrales. En effet, ses vastes dimensions induisent plusieurs contraintes.

Tout d'abord, le cadre de scène panoramique, difficile à maîtriser par les acteurs, peut entraîner une dispersion de l'image et donc de l'attention des spectateurs si on ne le réduit pas. La deuxième limite de cette salle est la distance entre la scène et le dernier rang de spectateurs s'élevant à 35 mètres alors que « les normes établies depuis plus de 25 ans par les architectes spécialistes fixent cette distance à 25 mètres maximum $[\ldots] \aleph^{51}$. Cette distance est un obstacle à la visibilité et empêche qu'existe une véritable communication entre la scène et la salle. La jauge élevée de la salle (2774 places $\left.{ }^{52}\right)$ est remise en cause par la pratique, mais aussi par ces mêmes normes qui définissent la jauge idéale entre 1300 et 1500 spectateurs. Quant à l'acoustique, il faut, pour l'améliorer, recourir à des appoints sonores. Enfin, cette salle se caractérise par l'inexistence d'équipements techniques devant le cadre de scène, sur les murs et le plafond du fait de sa décoration.

La salle montre également, dès les années 1960, des signes de vétusté: chauffage insuffisant, ventilation défectueuse, installation électrique dangereuse, installation électro-acoustique usée, affaissement d'une partie du balcon, sièges à remplacer, peinture à refaire... ${ }^{53}$ Cette situation nécessite donc d'intervenir.

En parallèle des réflexions menées pour une petite salle au sein du Palais de Chaillot, des travaux sont prévus dans la grande salle afin d'améliorer son état. Les courriers échangés entre 1963 et 1972 par l'administrateur du TNP et sa tutelle en attestent. En novembre 1964, une note liste les aménagements techniques qui ont eu lieu dans la salle durant la fermeture de l'été: la réfection du jeu d'orgues qui datait de la construction du théâtre; la modernisation de la cabine cinéma; l'installation d'une nouvelle console d'enregistrement comportant trois magnétophones professionnels dans la cabine son ; l'aménagement d'une nouvelle fosse d'orchestre sous le proscenium qui pourra être utilisée sans réduire la surface de jeu ${ }^{54}$.

Des projets de plus grande envergure au sein de la grande salle sont envisagés durant la direction de Georges Wilson auxquels Jacques Le Marquet participe activement. Ils sont chaque fois un peu plus ambitieux.

D’avril 1963 à décembre 1964, il étudie pour Georges Wilson la possibilité de réduire la jauge de la salle: une avancée du proscenium permettrait de supprimer 300 sièges, 120 devant la scène et 90 de chaque côté du parterre. La jauge atteindrait alors 2350 spectateurs. ${ }^{55}$

42 La seconde étude date d'avril 1967. La proposition de J. Le Marquet est plus précise et concrète, se matérialisant par un document de synthèse intitulé « Reconsidération de la Grande salle de Chaillot» daté du 22 avril 1967. Ce document de cinq pages mêlant notes explicatives et croquis, propose de reconsidérer, à titre provisoire, certaines proportions salle-scène (largeur du cadre de scène, profondeur du balcon) dans l'attente d'une refonte architecturale complète de la grande salle. "L'affaire sera traitée comme un décor de théâtre, ou comme le prolongement d'un décor dans la salle. Construction légère, peu durable quoique conforme aux règles de la sécurité publique [...], les sorties seraient intégralement respectées et l'ensemble "accroché" aux murs de la salle sans le moindre préjudice $»^{56}$, écrit Le Marquet.

43 La largeur de la salle serait réduite à 15 ou 16 mètres à l'aide de deux faux murs de 8 ou 9 mètres de haut qui partiraient des coins du balcon et rejoindraient le bord de la 
scène. « 90 places sauteraient de chaque côté " ${ }^{57}$. Les sorties latérales de la salle seraient maintenues, "mais légèrement allongées en profondeur $"^{58}$. Les faux murs seraient dans un matériau sombre, rigide et ininflammable. La profondeur du balcon serait quant à elle réduite de cinq rangs par un dispositif mobile de 3 ou 4 mètres de haut, s'étendant sur toute la largeur de la salle. Ce dispositif serait dans le même matériau que celui des faux murs. D'après Le Marquet - et ses archives le confirment -, ce dispositif est " fidèle ${ }^{59}$ à ce que Camille Demangeat avait proposé pour le Petit Festival de Suresnes en novembre $1951^{60}$.

Une troisième proposition, non datée, s'ensuit, « variante réorientant les blocs de côté du parterre autour d'un proscenium avancé sur les cinq premiers rangs $[. ..] \rrbracket^{61}$.

En juin 1968, J. Le Marquet propose un quatrième projet visant à insérer une salle audessus de la salle actuelle, une "scénographie en prothèse " $^{62}$ selon ses termes. Les principes directeurs du projet sont de ramener le dernier rang de 33 à 23 mètres du bord de scène, d'abaisser la jauge à 1600 places, de supprimer l'appoint sonore, devenu alors inutile. "Ainsi », selon Jean Ruaud, "disposerait-on d'une salle aux dimensions plus humaines et le problème du répertoire se trouverait de beaucoup simplifié, la démesure actuelle étant éliminée $»^{63}$.

La scène serait peu modifiée: le cadre de scène serait supprimé au moyen d'une décoration simple; il y aurait une avancée de deux mètres par rapport au bord du plateau. On construirait une salle autonome à l'intérieur de la grande salle, une salle " différente » marquant « une volonté de rupture " $^{64}$. "La salle actuelle est immense et sombre, celle-ci est ramassée et claire $»^{65}$. Cette salle en hémicycle partiel comprendrait deux niveaux desservis par le fond de la salle; le second niveau serait en porte-à-faux au-dessus du premier. Ces travaux de remodelage sont envisagés comme provisoires de façon à ne pas supprimer le vaste auditorium qu'offre la salle. On pourrait, si besoin, démonter ce dispositif pour retrouver l'ancienne salle. Une maquette en volume et de nombreux plans illustrent ce projet. 
Figure 4

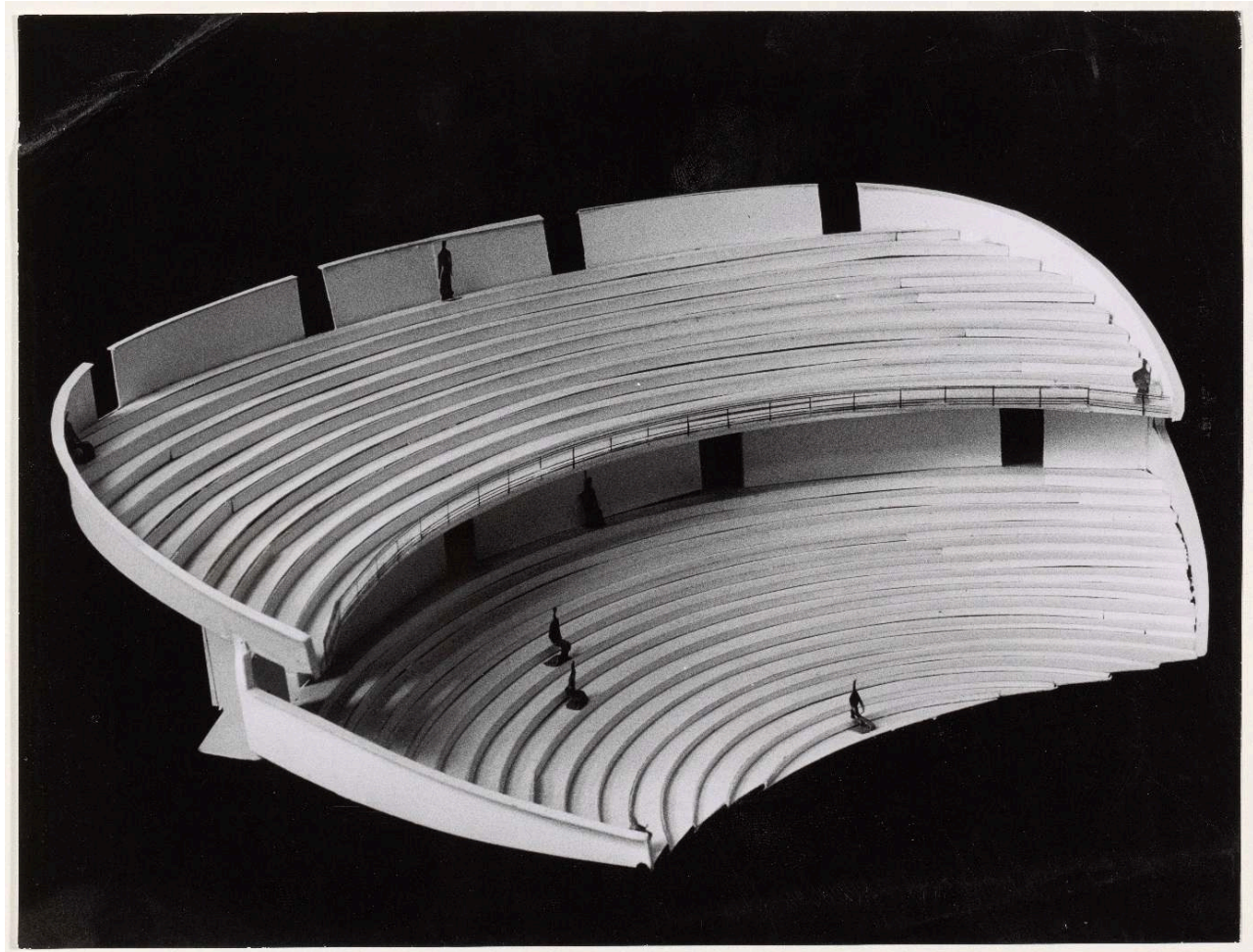

BnF, ASP, 4-COL-53 (148, 7), maquette en volume de Jacques Le Marquet (photographie de Roger Pic)

47 Ce projet, validé par Georges Wilson et Jean Ruaud, est exposé à Jean de Mailly et ses équipes. Une réunion les rassemble le 25 juillet 1968 pour mettre au point de façon définitive «la réforme de la grande salle du TNP pour la saison 1968/69 " ${ }^{66}$. Le compte rendu de cette réunion dressé par Le Marquet décrit précisément la salle dans sa structure et dans ses composantes (dimensions, matériaux et revêtements utilisés, accès, sièges, éclairage, chauffage, ventilation, décoration), ainsi que les travaux à effectuer. Le projet est inscrit par Jean Ruaud comme l'un des travaux importants à réaliser lors de la fermeture estivale qu'il prévoit de financer avec les crédits alloués par le ministère pour les travaux d'équipement du TNP (1 $250000 \mathrm{~F})$ : $150000 \mathrm{~F}$ seraient attribués aux travaux indispensables, $1100000 \mathrm{~F}$ à la grande salle. À l'été 1968, ce projet semble donc en bonne voie de concrétisation jusqu'à une note de J. Ruaud adressée à François Miermont, sous-directeur du Théâtre et des Maisons de la Culture, à la date du 26 septembre 1968 : le projet de remodelage de la grande salle a dû être abandonné ${ }^{67}$. Aucune explication n'est donnée ni dans les archives de J. Le Marquet, ni dans celles du TNP, ni dans celles de l'agence Jean de Mailly.

Deux ans plus tard, en 1970, J. Le Marquet participe à l'élaboration du programme architectural qui sera inscrit dans le $\mathrm{VI}^{\mathrm{e}}$ plan. Outre la refonte complète de la grande salle, solution "bâtarde $»^{68}$ qui aboutirait à un "compromis entre l'auditorium et le véritable théâtre moderne $»^{69}$, ce programme prévoit la construction d'une troisième salle enfouie dans les jardins du Trocadéro devant l'aile droite du palais (aile Passy). Cette salle aurait une audience de 500 à 1500 places. On pourrait y donner « toutes les représentations possibles, de toutes les formes de spectacles possibles et dans toutes les variantes d'organisations de public possibles. [...] Pour cela, sans notion distincte de salle et de scène, et autour d'un seul volume sans points porteurs, il faudrait créer un 
environnement technique "modelable" dans les trois dimensions : murs, sols, plafond [...] $\gg^{70}$. Ce projet ne fait l'objet d'aucun plan conservé dans les archives de Le Marquet. Sans avoir de suite, il annonce néanmoins le projet de salle modulable lancé par Jack Lang $^{71}$ en mai 1972 auquel il associe Le Marquet dont il connait le projet d'hémicycle pour la grande salle. Le Marquet accepte car le projet de Jack Lang l'intéresse " pour la raison qu'il correspond exactement à [ses] conceptions de la scénographie architecturale contemporaine $\aleph^{72}$. Aux côtés des architectes Valentin Fabre (Atelier d'urbanisme et d'architecture) et Christian du Pavillon, de Noël Napo (directeur technique de Jack Lang), Le Marquet participe à la phase d'élaboration du projet, secondé par Pierre Saveron (pour l'équipement électro-acoustique) et André Bataille. Entre le 31 mai et le 8 juin, il élabore plusieurs séries de plans qu'il soumet à Lang. Le 7 juin, il lui remet dix-sept plans sur calque qui sont essentiels pour l'établissement du cahier collectif du programme. Ces plans simulent, en plans et en coupes, les différentes possibilités d'organisation de l'espace scène-salle que permettent scène mobile, tribunes mobiles et diaphragmes ${ }^{73}$. À ce stade, le travail de Le Marquet est «terminé. Les autres mettent au point le petit cahier anonyme avant le lundi 12 juin, jour où Jack Lang doit le remettre aux Arts et Lettres $»^{74}$.

Figure 5

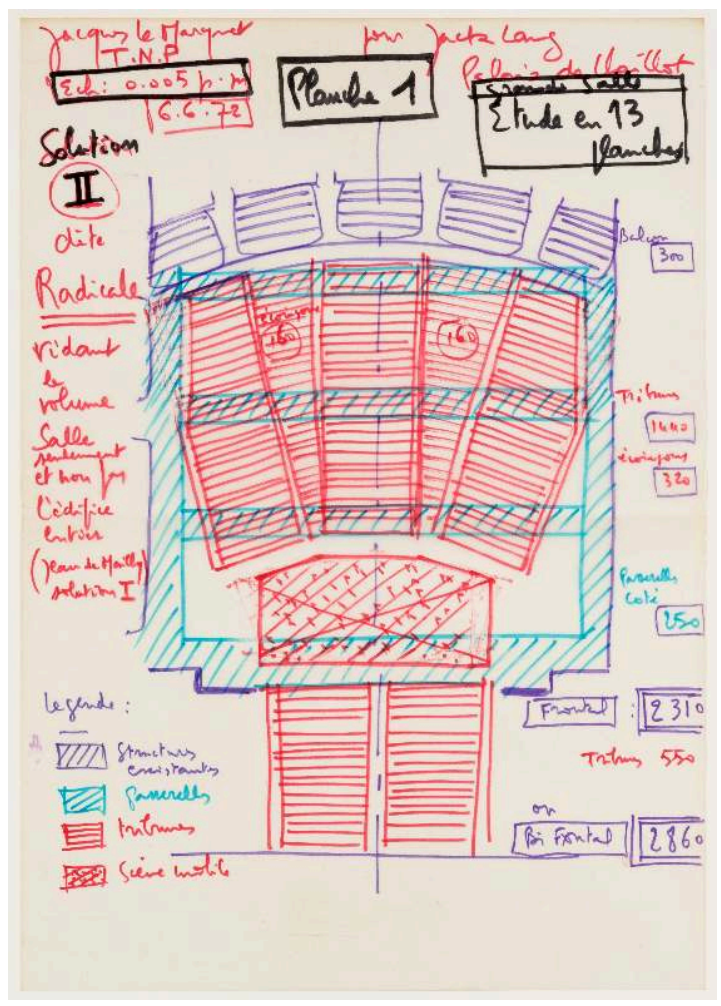

BnF, ASP, 4-COL-53 (162, 4), recherches sur calque de Jacques Le Marquet, 6 juin 1972

Durant les mois qui suivent, Le Marquet essaie d'avoir des renseignements sur l'avancée du projet, mais sans succès. À l'automne, il apprend que l'AUA travaille, que le projet est chiffré à 4 milliards de francs et vingt-six mois de travaux. À cette époque, le licenciement des agents du TNP commence. Le 19 octobre, Jack Lang met fin aux fonctions de régisseur de Le Marquet mais souhaiterait qu'il travaille pour lui sur un projet de salle provisoire. Le Marquet accepte et commence à faire des propositions de 
plans. Le 6 novembre, Noël Napo lui dit que finalement ses propositions ne les intéressent pas. La collaboration s'arrête. À l'issue du licenciement des cadres du TNP (22 novembre 1972), les plans des travaux de la grande salle sont affichés et rendus publics : ils sont tirés du cahier élaboré en juin à la conception duquel Le Marquet a participé, sans être cité, ni rétribué. Il gardera de cet épisode une «certaine irritation $»^{75}$.

Le Marquet a donc joué un rôle essentiel dans les projets affectant les espaces de jeu du TNP. Il a été l'intermédiaire technique des architectes, sans que sa participation soit officiellement reconnue. Ces activités annoncent sa collaboration avec des architectes dans la construction de salles de spectacles, et notamment sa collaboration avec Jean Nouvel pour lequel il travaillera de 1975 à 2008.

\section{Conclusion}

51 Au-travers de l'exemple de Jacques Le Marquet, nous avons pu appréhender comment un membre de l'équipe du TNP a travaillé dans les espaces du Palais de Chaillot et comment il les a utilisés. J. Le Marquet et ses collègues ont rapidement perçu les potentialités et les limites de ces espaces et ont essayé, à chaque occasion, d'en tirer le meilleur parti. Leur ingéniosité et leur talent ont permis de pallier les obstacles rencontrés et d'offrir au public des soirées inoubliables ${ }^{76}$.

Les archives conservées à la Bibliothèque nationale et aux Archives nationales documentent ce qui est resté dans la mémoire collective à la fois comme une très grande aventure théâtrale - le TNP - et comme un cadre difficile, sinon impossible à exploiter - le Palais de Chaillot.

\section{NOTES}

1. Jacques Le Marquet, Introduction à la rubrique Théâtre, (BnF, Arts du spectacle (ASP), FOLCOL-53 $(248,1)$, p. 6 . Ces documents sont des maquettes originales de peintres ou de scénographes qui lui ont été confiées pour la réalisation de leur(s) décor(s) [Léon Gischia, Yves Saint-Laurent, Mario Prassinos], des dessins de Jean Vilar, Georges Wilson et Camille Demangeat. 2. Fonds Théâtre national populaire - Direction Georges Wilson, 1963-1972 (BnF, Arts du spectacle (ASP), 4-COL-44) ; archives de Jacques Le Marquet (BnF, ASP, 4-COL-53) ; archives de l'agence Jean de Mailly (Archives départementales des Hauts-de-Seine).

3. BnF, ASP, 4-COL-53 $(1,1)$, Jacques Le Marquet, croquis-portrait $n^{\circ} 2$ consacré à Jean Vilar, 1999.

4. Camille Demangeat (1905-1985) entre au service de Louis Jouvet au théâtre de l'Athénée avant la guerre. De 1941 à 1945, il le suit en tournées comme chef-constructeur. En 1947, il rejoint la troupe de Jean Vilar et en crée tous les dispositifs scéniques, puis ceux du TNP jusqu'en 1954. Après son départ du TNP, il fonde la première agence spécialisée en scénographie d'équipement [BnF, ASP, 4-COL-53 (6)].

5. Pan de bois constitué de piliers verticaux et de poutres horizontales. [...] Dans les théâtres à l'italienne on rencontre trois utilisations essentielles de fermes, les fermes des dessous, les 
fermes de décor et les fermes de praticable. Cette définition et les autres définitions de termes techniques sont tirées du Dictionnaire raisonné et illustré du théâtre à l'italienne d'Alain Roy, Actes Sud, Arles, 2001, $134 \mathrm{p}$.

6. Fermes en battants qui supportent le plancher des praticables construits spécifiquement pour un décor.

7. On désigne communément les deux ailes incurvées du Palais de Chaillot descendant vers les jardins du Trocadéro, aile Paris et aile Passy, la première allant en direction de Paris et la seconde en direction du quartier de Passy.

8. Les informations relatives à l'emplacement de ces bureaux et espaces nous ont été données par Nicolle Daviot qui a été habilleuse-couturière pour le théâtre de Chaillot de 1967 à 2008 (entretien du 28 juin 2018).

9. BnF, ASP, 4-COL-53 $(2,6)$, Jacques Le Marquet, croquis-portrait $n^{\circ} 14$ consacré à Claude Jouen.

10. Ibid., croquis-portrait $n^{\circ} 13$ consacré à Raymond Jousselin.

11. Ibid.

12. BnF, ASP, 4-COL-53 $(162,6)$, «Propositions de travaux à effectuer au T.N.P. dans le cadre du $\mathrm{VI}^{\mathrm{e}}$ plan »,1970, p. 2. Ce texte n'est pas signé mais nous pensons qu'il émane de la direction du TNP et que J. Le Marquet a participé à sa rédaction (un texte de lui y est cité).

13. BnF, ASP, 4-COL-53 (160, 3), note de service de Jacques Le Marquet, 5 mai 1971.

14. Ibid.

15. BnF, ASP, 4-COL-53 (148, 1), note de Jacques Le Marquet, 6 octobre 1999.

16. Ibid.

17. BnF, ASP, 4-COL-53 (134, 1), note rétrospective de Jacques Le Marquet, 3 mars 1999, p. 1.

18. Les informations sur le Théâtre Récamier sont tirées de l'ouvrage d'Emmanuelle LOYER, Le Théâtre citoyen de Jean Vilar. Une utopie d'après-guerre, Paris, P.U.F., 1997, p. 223-225.

19. BnF, ASP, 4-COL-53 (134, 1), note rétrospective de Jacques Le Marquet, 3 mars 1999, p. 2.

20. BnF, ASP, 4-COL-53 (134, 3), note de Lucien Fresnac à Jacques Le Marquet, 27 mai 1963, p. 1.

21. Georges Wilson l'a contacté pour ses connaissances en scénographie d'équipement.

22. BnF, ASP, 4-COL-53 (134, 1), note rétrospective de Jacques Le Marquet, 3 mars 1999, p. 2.

23. BnF, ASP, 4-COL-53 (134, 6), lettre de Jacques Le Marquet à Louis Blanchet, 28 juin 1963, p. 2.

24. Ibid., p. 1.

25. BnF, ASP, 4-COL-53 $(134,1)$, note rétrospective de Jacques Le Marquet, 3 mars 1999, p. 3

26. Cette information est tirée de l'interview de Jean de Mailly par J.-C. Jaubert dans la revue Bref, n 91, décembre 1965, p. 22-23. La fresque de Raoul Dufy fut déposée au musée national d'Art moderne et est exposée aujourd'hui au musée des Beaux-Arts de Lyon. La fresque d'Othon Friesz est aujourd'hui conservée par la Médiathèque de l'architecture et du patrimoine à Charenton-lePont ; nous ignorons dans quelle maison de la culture elle a été conservée avant la création de cet établissement (1996).

27. Il remplace Lucien Fresnac, mort le 19 septembre 1963, qui avait suivi le début du projet.

28. Rideau tendu qui est circulaire, ou plutôt semi-circulaire. Il se développe entre les murs cour, lointain et jardin.

29. BnF, ASP, 4-COL-53 (143, 2), lettre de Jean Ruaud à Jean de Mailly, 12 novembre 1966.

30. Ibid., note de service de Jean Ruaud, 24 novembre 1966.

31. BnF, ASP, 4-COL-44 (81, 7), note de Jean Ruaud, 9 avril 1968.

32. Toutes les informations sur la salle Gémier qui suivent, hormis les citations de Jean de Mailly, sont tirées de la «Fiche technique. Rapport de scénographie ", conservée dans le fonds Théâtre national populaire - Direction Georges Wilson (1963-1972), 4-COL-44 (81, 2).

33. BnF, ASP, 4-COL-53 $(143,1)$, Jean de Mailly, «Paris. Palais de Chaillot. Petite salle du T.N.P.» dans Techniques et Architecture, n 6, mars 1967.

34. Archives départementales des Hauts-de-Seine, fonds de l'Agence Jean de Mailly, $28 \mathrm{~J}$, boîte 1, document non daté sur les surfaces des salles de spectacle du Palais de Chaillot. 
35. Partie avancée de la scène qui envahit le parterre, de telle sorte qu'il se trouve entouré de spectateurs ce qui est plus spécifiquement italien que français. En Italie, ce proscenium est solidaire de l'ensemble de la scène, tandis qu'en France il est généralement amovible [...]. Ce proscenium occupe, quand il est monté, l'emplacement des deux à cinq premiers rangs.

36. Jean de Mailly, propos recueillis par J.-C. Jaubert pour l'article «Demain, la petite salle du T.N.P. » dans Bref, nº 91, décembre 1965, p. 22.

37. Ce terme évoque, par opposition à la face, le fond de la scène et surtout du décor.

38. Dispositif d'éclairage placé sur le plancher de l'avant-scène, tout le long du nez-de-scène.

39. Partie aérienne de la cage de scène. Dans cet espace se meuvent les fils, les perches et les porteuses. Les cintres sont entourés d'une ou plusieurs passerelles de service et sont traversés par un ou plusieurs ponts volants.

40. Plancher à claire-voie qui doit son nom à cette particularité le faisant ressembler à un gril, instrument culinaire. Il est suspendu au-dessus et juste à l'aplomb du plancher de la cage de scène [...] et a pour fonction de supporter tous les éléments suspendus du décor.

41. «Janvier 1967 : une petite salle au T.N.P.» dans le dossier "Brève histoire de Bref », textes commentés par Jean-Claude Marrey, dans Bref, n 101, décembre 1966, p. 31.

42. Propos de la directrice du C.E.G., 56 ans recueillis pour l'article « La salle Gémier vue par les premiers spectateurs. Son rôle et son aspect », dans Bref, n 103, février-mars 1967, p. 16.

43. Ibid., propos d'un graphiste de 34 ans.

44. Ibid.

45. Ibib., propos d'un professeur de 25 ans.

46. Ibid., propos d'un journaliste de 26 ans.

47. Ibid., propos d'un élève-professeur de 24 ans.

48. BnF, ASP, 4-COL-53 $(143,1)$, note rétrospective de Jacques Le Marquet, "La salle Gémier ", 29 septembre 1999.

49. Ibid.

50. L'article de Sandrine Dubouilh, «Les archives d'architecture : comprendre ce que fut l'outil du TNP à Chaillot ", qui précède le nôtre, donne davantage d'informations sur la grande salle (construction, implantation et caractéristiques).

51. BnF, ASP, 4-COL-53 $(162,6)$, « Propositions de travaux à effectuer au T.N.P. dans le cadre du $\mathrm{VI}^{\mathrm{e}}$ plan $»(1970), \mathrm{p} .1$.

52. Cette jauge peut être réduite à 2650 places pour les représentations du TNP en recouvrant les quatre premiers rangs par un proscenium volant.

53. BnF, ASP, 4-COL-53 (162, 6), « Théâtre du Palais de Chaillot. Aménagement. Juin 1972 », p. 4.

54. BnF, ASP, 4-COL-44 $(81,5)$, note de novembre 1964. Cette note n'est pas signée mais quelques notes manuscrites y figurent qui semblent être de la main de Jean Ruaud.

55. BnF, ASP, 4-COL-53 $(148,2)$, plans sur calque de Jacques Le Marquet.

56. BnF, ASP, 4-COL-53 (148, 3), « Reconsidération de la Grande salle de Chaillot », 22 avril 1967,

p. 1.

57. Ibid., p. 2.

58. Ibid., p. 3.

59. Ibid., note rétrospective en rouge de Jacques Le Marquet, p. 1.

60. Dans les archives de Jacques Le Marquet $(4-\mathrm{COL}-53(8,1)$ et $(8,2)$, Tournée en France : Petit Festival de Suresnes, automne 1951), un article de Camille Demangeat illustré d'un croquis présente ce dispositif et une aquarelle le donne à voir.

61. BnF, ASP, 4-COL-53 $(148,4)$, note de Jacques Le Marquet non datée.

62. BnF, ASP, 4-COL-53 (162, 1), Jacques Le Marquet, «Exercices de la scénographie des espaces de jeu malléables », p. 2.

63. BnF, ASP, 4-COL-44 (81, 7), note de Jean Ruaud, 1968, p. 1. 
64. BnF, ASP, 4-COL-53 $(148,5)$, compte rendu de la réunion de travail du 25 juillet 1968 fait par Jacques Le Marquet, p. 2. Cette référence vaut également pour l'adjectif « différente ».

65. Ibid.

66. Ibid., p. 1.

67. La plupart des informations de ce paragraphe sont tirées du fonds Théâtre national populaire - Direction Georges Wilson (1963-1972), 4-COL-44 (81, 7).

68. BnF, ASP, 4-COL-53 $(162,6)$, « Propositions de travaux à effectuer au T.N.P. dans le cadre du VI ${ }^{\mathrm{e}}$ plan $", 1970$, p. 4.

69. Ibid.

70. Ibid., p. 5. Les principes de cette troisième salle sont tirés du sujet soumis par J. Le Marquet aux étudiants de l'École nationale supérieure des arts décoratifs pour l'obtention de leur diplôme de fin d'année en 1969.

71. Jack Lang est nommé directeur du Théâtre national de Chaillot en avril 1972, mais ne prend ses fonctions qu'en octobre.

72. BnF, ASP , 4-COL-53 $(163,1)$, lettre de Jacques Le Marquet au délégué des cadres et agents de maîtrise du Palais de Chaillot concernant l'indemnité de licenciement, 6 novembre 1972, p. 2. Il ajoute: "Dans ce sens, cette matière que j'enseigne aux Arts décoratifs depuis quatre ans a constitué le matériel essentiel des diplômes de fin d'études ".

73. BnF, ASP, 4-COL-53 (162, 4), recherches sur calque de Jacques Le Marquet, 23 mai-6 juin 1972, 13 planches.

74. BnF, ASP, 4-COL-53 (162, 1), «Chronologie des événements » par Jacques Le Marquet, p. 4.

75. BnF, ASP, 4-COL-53 (162, 1), note rétrospective de Jacques Le Marquet, 3 mars 1999.

76. Lors de notre communication, ainsi que dans cet article qui la reprend en l'étoffant, nous n'avons pas évoqué l'activité de scénographe de Jacques Le Marquet qui, entre 1961 et 1972, a conçu vingt-deux scénographies pour les salles du Palais de Chaillot, dix-sept pour la grande salle et cinq pour la salle Gémier. Par ses scénographies, il a participé à rendre les soirées du TNP inoubliables. Pour plus d'informations sur l'activité de scénographe de théâtre de J. Le Marquet, voir notre thèse de l'École des chartes, Jacques Le Marquet, scénographe aux multiples talents (1953-1990), 2007 et notre thèse de doctorat thèse en « Communication, arts et spectacles ", Jacques Le Marquet (1927-2017), une conjonction de talents et de pratiques au service de la scénographie, menée sous la direction de Mme Sandrine Dubouilh Frechet, université de Bordeaux Montaigne, 2019. https://www.theses.fr/s182735

\section{RÉSUMÉS}

Jacques Le Marquet (1927-2017) a travaillé vingt ans dans le Palais de Chaillot au sein du Théâtre National Populaire (TNP) : au travers de son exemple, il nous est possible d'appréhender la façon dont le TNP a composé avec les espaces du Palais de Chaillot et la façon dont un de ses membres a travaillé dans et avec ces espaces.

Entré au TNP en 1952 comme second adjoint constructeur, J. Le Marquet succède à Camille Demangeat à la tête de la régie Construction en 1954, et assure cette fonction jusqu'à son départ en octobre 1972. À ce titre, il est l'intermédiaire entre le scénographe - concepteur de décors et les équipes chargées de réaliser décors et accessoires. Il est amené à dresser des plans de décors et, une fois ces derniers construits, à en surveiller l'installation sur scène. Il est également chargé 
d'organiser les tournées et le Festival d'Avignon pour ce qui incombe à son service. Du fait de ces activités et des compétences qu'il a développées dans le domaine de la scénographie d'équipement, J. Le Marquet est associé aux projets de réaménagement et de construction relatifs aux espaces occupés par le TNP. Il va notamment jouer un rôle essentiel dans l'élaboration de la salle Gémier (1963-1966) et, va faire, entre 1963 et 1972, plusieurs propositions de réaménagements de la grande salle, dont les dimensions sont un obstacle quotidien au bon fonctionnement du théâtre.

\section{INDEX}

Mots-clés : Théâtre national populaire, Palais de Chaillot, Le Marquet (Jacques), Vilar (Jean), Wilson (Georges)

\section{AUTEUR}

\section{ANAÏS DUPUY-OLIVIER}

Conservatrice au département des Manuscrits de la BnF, docteur en Études théâtrales, université de Bordeaux 


\title{
Jeanne d'Arc au bûcher au Palais de Chaillot de 1939 à 1942 : une simple reprise?
}

\author{
Pascal Lécroart
}

1 La riche histoire du théâtre du Palais de Chaillot, au moins depuis sa reconstruction en 1937 selon les plans des frères Niermans, a souvent rencontré l'Histoire, parfois pour le meilleur - la signature de la Déclaration universelle des droits de l'homme le 10 décembre 1948 -, et parfois pour le pire - un rassemblement du parti nazi en octobre $1942^{1}$. Officiellement inauguré le 24 février 1939, le Théâtre du Palais de Chaillot a été placé sous la direction de Paul Abram, seulement six mois avant que n'éclate la Seconde Guerre mondiale. L'Occupation le marque d'emblée de son empreinte : son directeur est révoqué par Vichy comme juif et il sera obligé de vivre caché pendant toute cette période ${ }^{2}$. Il est remplacé par Pierre Aldebert (1889-1966) qui, cas singulier par rapport aux théâtres nationaux, n'aura pas à démissionner à la Libération malgré l'utilisation de la salle pour des activités collaborationnistes. Il restera directeur jusqu'à la nomination de Jean Vilar en 1951. Le fonctionnement propre de Chaillot explique vraisemblablement cette exception : à l'inverse de l'Opéra, de la Comédie-Française et de l'Odéon, la salle n'est pas soumise de manière globale à une programmation établie pas son directeur qui en porterait la responsabilité. Le Théâtre du Palais de Chaillot, aux moyens financiers totalement insuffisants, donne, pendant l'Occupation, quelques spectacles originaux, presque toujours dans des mises en scène de Pierre Aldebert. Outre de nombreux concerts, Chaillot accueille, de manière impersonnelle, des spectacles montés ailleurs qui trouvent, dans ce lieu, une destination plus ouverte et plus populaire, dans l'héritage de la politique menée depuis 1920 avec la direction de Firmin Gémier. Après la Libération, la salle propose différents galas de soutien au bénéfice des combattants, des résistants et des alliés.

2 Pendant l'Occupation, Chaillot est surtout un grand lieu de la vie musicale parisienne. Malgré son acoustique jugée d'emblée peu valorisante, la salle, avec ses 2800 places, offre une jauge supérieure à celle de la Salle Pleyel, limitée à 2500 places environ ${ }^{3}$, dont l'acoustique fait également l'objet de critiques. Chaillot a ainsi régulièrement accueilli 
de grands concerts d'orchestres allemands, en tournées de propagande, sur des programmes entièrement germaniques. Dès juin 1941, le jeune chef d'orchestre Herbert von Karajan y dirige un concert de l'orchestre de l'Opéra de Berlin dans un programme Wagner, en présence de Winifried Wagner, veuve de Siegfried Wagner, fils de Richard, et sympathisante nazie de la première heure; l'événement fait l'objet d'un reportage pour le journal cinématographique ${ }^{4}$. C'est le début d'une longue série : le 17 juillet 1944, l'Orchestre philharmonique de Berlin, placé sous la direction de Hans Knappertsbusch dans un programme Beethoven, Brahms et Wagner, fait encore salle comble ${ }^{5}$. Parallèlement, Chaillot accueille aussi les grandes manifestations de l'Orchestre de la Société des concerts du Conservatoire, la vieille salle du Conservatoire, rue de Madrid, ayant une jauge désormais trop limitée (moins de 1000 places). Son directeur, Charles Münch, y défend vigoureusement le répertoire français et tente, sur le fil, malgré son exposition, d'éviter toute compromission collaborationniste. Membre du Front national - mouvement de la Résistance -, il recevra la Légion d'honneur en 1945 pour ses activités clandestines en faveur des musiciens. De ce point de vue, Chaillot offre un visage contrasté et s'oppose au Théâtre des Champs-Élysées, lieu d'exercice de l'Orchestre de Radio-Paris, son principal concurrent, à l'inscription collaborationniste nettement plus uniforme.

On connaît les débats multiples et les polémiques suscités par tous les travaux historiques concernant l'Occupation allemande et les arts. Depuis presque vingt ans, de nombreuses études ont paru concernant la musique et les musiciens pendant cette période. Cet article tentera de s'inscrire dans ce vaste panorama à travers la présentation et l'analyse d'un cas précis: celui des deux exécutions de Jeanne d'Arc au bûcher, oratorio-dramatique de Claudel et Honegger, d'emblée perçu comme une réalisation capitale à l'époque. Chaillot a en effet accueilli sa création parisienne le 13 juin 1939, puis sa reprise importante presque trois ans plus tard, le 25 juin 1942, en pleine Occupation. Quel sens a-t-on alors donné à cet événement? Après avoir retracé le contexte général qui a présidé à la naissance de cette œuvre et à cette création parisienne de 1939, j'étudierai, à partir de différents documents d'époque, le positionnement ambigu et complexe de cette apparente reprise ${ }^{6}$.

\section{Le contexte de la création parisienne au Palais de Chaillot}

4 La création parisienne de Jeanne d'Arc au bûcher de Claudel et Honegger ${ }^{7}$ au Palais de Chaillot, le 13 juin 1939, tient presque du hasard. L'œuvre avait été originellement commandée par la mécène, danseuse et comédienne Ida Rubinstein ${ }^{8}$ en 1934 à l'écrivaine Jeanne d'Orliac et au compositeur Arthur Honegger. Elle devait s'inspirer des représentations de théâtre médiéval proposées par les étudiants de la Sorbonne, sous l'égide du médiéviste Gustave Cohen, avec la participation de Jacques Chailley qui venait de fonder le chœur de la Psalette Notre-Dame. Mais le travail de l'écrivaine ne convient pas : Ida Rubinstein se tourne alors vers Paul Claudel qui écrivait pour elle une œuvre d'inspiration biblique, La Sagesse ou la parabole du festin, dont Darius Milhaud composait la musique. Le texte de Jeanne au bûcher est rédigé à la fin de 1934, tandis qu'Honegger construit sa partition entre mars et décembre 1935. Les représentations des deux œuvres auraient dû avoir lieu à l'Opéra de Paris, mais se trouvent sans cesse repoussées par la mécène, au grand dam des auteurs. Finalement, l'œuvre est créée en 
Suisse alémanique, à Bâle, le 11 mai 1938, sous la direction de Paul Sacher, avec Ida Rubinstein dans le rôle-titre. À la suite du succès de cette création, la mécène accepte que l'œuvre soit donnée en création française à Orléans, le 8 mai 1939, lors des Fêtes Jeanne d'Arc, à l'initiative de l'évêque d'Orléans, $\mathrm{M}^{\mathrm{gr}}$ Courcoux, en présence du nonce Valerio Valeri et de Jean Zay, ministre de l'Éducation nationale. L'Orchestre philharmonique de Paris assure cette création française sous la direction de Louis Fourestier avec les chœurs de Félix Raugel.

5 Le succès et le soutien officiel des représentants de l'État a permis une reprise très rapide à Paris, le 13 juin 1939, dans la salle du Palais de Chaillot alors disponible, avec les mêmes interprètes dont Jean Hervé dans le rôle de Frère Dominique. La comparaison de l'en-tête des programmes montre l'évolution du cadre: en mai, il n'était question que des «Fêtes traditionnelles de Jeanne d'Arc» de la «ville d'Orléans ", là où la création parisienne mentionne que l'œuvre est donnée «Sous le haut patronage de M. le Président de la République, sous la présidence d'honneur de M. Georges Bonnet, Ministre des Affaires étrangères, et de M. Jean Zay, Ministre de l'Éducation nationale, sous la présidence de M. le Professeur Gustave Roussy, Recteur de l'Académie de Paris, et de M. Georges Huysman, directeur général des Beaux-Arts ». C'est presque une création officielle pour une œuvre écrite sur un sujet à dimension nationale. Pour l'occasion, on s'est servi, en fond de scène, des importants éléments de décor réalisés par Alexandre Benois - qui a ainsi sa place dans le programme - en vue des représentations à l'Opéra de Paris. Cela confère un prestige supplémentaire à cette première parisienne. De manière presque simultanée, Paul Sacher a dirigé une reprise de l'œuvre à Bâle puis à Zurich, les 10 et 11 mai 1939, toujours avec la mécène dans le rôle principal et Jean Hervé à son côté. En l'espace d'un mois, Ida Rubinstein a donc assuré quatre exécutions de l'ouvrage. Les comptes rendus sont nombreux et extrêmement louangeurs pour la quasi-totalité d'entre eux, en allemand pour la Suisse alémanique, en français et même en anglais'. Le rayonnement de l'œuvre s'étend en 1940: le 22 février, Ida Rubinstein redonne l'ouvrage à la salle Pleyel avec, pratiquement, la même distribution qu'en juin 1939. Enfin, entre le $1^{\text {er }}$ et le 3 mars, Ida Rubinstein et Jean Hervé assurent la création en Belgique sous la direction de Louis de Vocht, avec trois exécutions à Bruxelles et une à Anvers.

Jeanne d'Arc était toujours, dans les années trente, un sujet particulièrement à la mode. Dans le domaine musical, on compte notamment la Messe pour le 500 anniversaire de la mort de Jeanne d'Arc de Paul Paray, créée à Rouen en 1931, Paysage pour une Jeanne d'Arc à Domrémy, œuvre symphonique de Jean Rivier (1936), la suite symphonique Jeanne d'Arc de Manuel Rosenthal, en 1936, d'après le roman de Joseph Delteil dont Maurice Ravel aurait voulu tirer un opéra, la cantate Jeanne d'Arc (1937), pour chœur et orchestre, de Roland-Manuel, dans le cadre du spectacle Liberté du Front populaire, la symphonie concertante Jeanne d'Arc de Maurice Jaubert pour soli, chœur et orchestre la même année. Cependant, dès sa création, Jeanne d'Arc au bûcher s'impose de manière particulièrement forte et, déjà, dans des cadres de réception très différents selon les lieux et les moments. La création en Belgique dans le contexte de guerre a, par exemple, donné lieu à l'expression d'une intense ferveur francophile naturellement inexistante lors de la création à Bâle. 


\section{La reprise à Chaillot en 1942}

7 La période de l'Occupation, qui constitue, à nos yeux et aux yeux des contemporains, une rupture profonde, n'empêche pas, sur le plan artistique, une forme de continuité : Jeanne d'Arc fait cette fois l'objet d'un véritable culte par Pétain et le régime de Vichy mais immédiatement disputé par la Résistance. L'héroïne nationale est, plus que jamais, à la mode, tout en étant l'objet d'un culte ambigu. En mai 1941, plusieurs villes de la zone non-occupée montent un immense spectacle collectif, Portique pour une fille de France, de Pierre Schaeffer et Pierre Barbier et, l'année suivante, sept écrivains et sept musiciens collaborent à une Jeanne d'Arc radiodiffusée ${ }^{10}$. Dans ce contexte, Jeanne d'Arc au bûcher se trouve récupérée par le régime de Vichy. Avec le soutien du Groupe Jeune France et à l'instigation de Pierre Schaeffer, l'œuvre est donnée à Lyon dans une mise en scène luxueuse de Pierre Barbier en juillet 1941, avant une tournée d'un mois par train dans 26 villes de la zone non-occupée. Pétain assiste à la première, à distance de Claudel qui est présent mais s'est brouillé avec le metteur en scène. Honegger, en revanche, qui n'a pas la moindre sympathie pour le régime de Vichy, n'a pas fait le déplacement. La presse salue, dans sa presque totalité, le choix de l'œuvre, symbolisant une forme de survivance et de renaissance possible de la grandeur de la France. Mais L'Action française se distingue : tout en louant la musique, le journal se moque du texte de Claudel et rappelle, de manière satirique, la création à Orléans "où Mme Ida Rubinstein innait [sic]... Jeanne d'Arc! Signe des temps! $»^{11}$.

8 La reprise dans le Paris occupé de 1942 offre un nouveau cadre d'interprétation. L'œuvre y est redonnée pour la première fois depuis les deux exécutions de 1939 et 1940 dans le cadre de la "Semaine Honegger »: un ensemble de quatre concerts consacrés au compositeur pour fêter son cinquantième anniversaire, allant de la musique de chambre à des œuvres symphoniques et chorales. Le premier est celui organisé au Palais de Chaillot le jeudi 25 juin 1942. Jeanne au bûcher y est précédée de la création de la Symphonie $n^{\circ} 2$ pour cordes, commande de Paul Sacher créée à Bâle un mois auparavant. Il s'agit, en même temps, du quatrième des concerts de gala qui font la clôture de la saison de l'Orchestre de la Société des concerts du Conservatoire, ouverte en octobre. Le programme en avait été particulièrement chargé :

9 - Jeudi 4 juin 1942 : festival Fauré-Debussy,

10 - Jeudi 11 juin 1942 : festival Bach-Françaix, proposant la création de son oratorio L'Apocalyse de saint Jean,

11 - Jeudi 18 juin 1942 : festival Beethoven, imposé à Charles Munch, avec le pianiste Wilhelm Kempt ${ }^{12}$.

12 Ces quatre concerts ont bénéficié d'un même cadre pour leur organisation. Dans l'entête du programme de la répétition générale ouverte aux étudiants ${ }^{13}$, il est clairement précisé : 


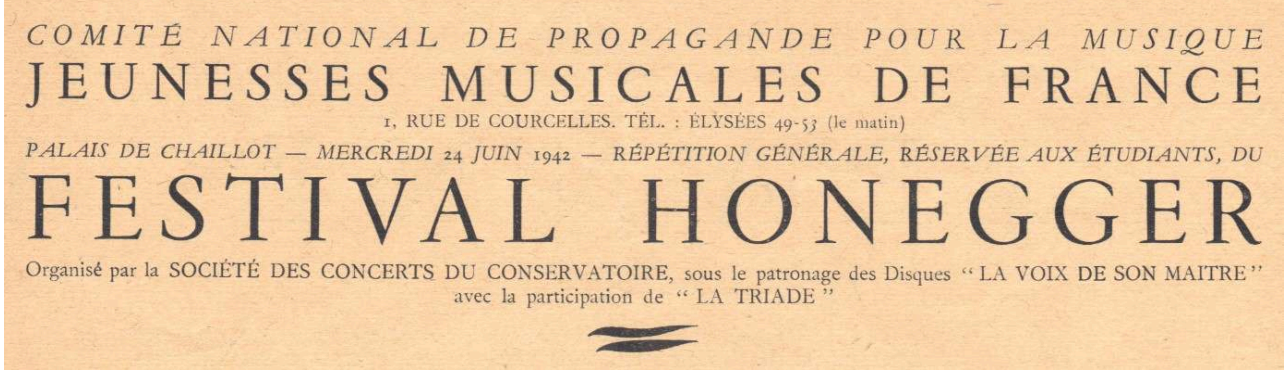

En-tête du programme du Festival Honegger

Collection privée

Le «Comité national de propagande pour la musique » avait été fondé en janvier 1931 avec cet objectif :

Engager les chefs de famille à cultiver chez leurs enfants le goût de la musique ; faire l'éducation musicale du public; donner à l'enseignement et à la culture de la musique parmi la jeunesse un caractère obligatoire sans qu'on puisse dire qu'il y ait contrainte, en ce sens que la musique doit procurer à l'enfant comme à l'homme adulte la détente indispensable à tout labeur intellectuel ou manuel ${ }^{14}$.

Vient ensuite la mention des "Jeunesses musicales de France » qui, fondées par René Nicoly, ont trouvé, dans ces quatre concerts, l'occasion de leur première manifestation : être membre de l'organisation permettait aux jeunes lycéens ou étudiants d'assister à la répétition générale pour un prix modique ${ }^{15}$.

Si les mentions précédentes étaient sans doute spécifiques au programme de la répétition générale, il n'en est pas de même des suivantes. La firme de disques « La voix de son maître " était très active en cette période: placée sous la direction de Jean Bérard, elle avait notamment assurée, en 1941, l'enregistrement mythique de Pelléas et Mélisande, dirigé par Roger Désormière et celui de La Danse des morts, oratorio également signé par Claudel et Honegger, avant celui de Jeanne d'Arc au bûcher en 1943. "La Triade », enfin, est une association fondée par la cantatrice Mona Péchenart en 1942, destinée à favoriser les relations entre les artistes ${ }^{16}$.

\section{Le programme de 1942}

Le contenu du programme est dû à Jacques Chailley. Il se compose d'une présentation de la Symphonie $n^{\circ} 2$ et de Jeanne d'Arc au bûcher. Chailley rappelle son implication dans la genèse de cet ouvrage et présente ensuite une courte analyse, scène par scène. Le contexte de l'Occupation parait d'abord totalement absent, mais on relève néanmoins quelques éléments qu'on pourra juger révélateur :

17 - la volonté d'insérer l'œuvre dans son époque antérieure, au cours des années 1930. Le programme rappelle les circonstances de sa composition depuis les représentations de théâtre médiéval par la troupe des "Théophiliens », formée d'étudiants de lettres de la Sorbonne, sans que le nom du professeur Gustave Cohen, médiéviste qui avait été son principal garant et son animateur, alors exilé aux États-Unis, n'apparaisse,

18 - la mention explicite, en revanche, de la commanditrice, Ida Rubinstein, qui était alors réfugiée en Angleterre et apportait son soutien à la Résistance gaulliste; on note au passage que le rôle de Frère Dominique est interprété, comme en 1939 et 1940, par Jean 
Hervé qui avait été mis à la retraite de la Comédie-Française quelques mois auparavant, manière d'évincer un acteur qu'on accusait d'être franc-maçon,

19 - quelques reformulations assez étonnantes dans le résumé de l'œuvre. La scène 5 donne lieu à cette présentation : “ 'C'est la politique, dit frère Dominique, qui l'a menée là". Et la détestable Politique apparaît, symbolisée par le Jeu de cartes.» Or, jamais Frère Dominique n'emploie le terme de "politique ». Pour la scène 8 , Chailley évoque : « les deux moitiés de la France, jadis séparées, et heureuses de se trouver enfin réunies; heureuses d'échapper à l'influence étrangère des Anglais et de pouvoir enfin "penser Français" ». Si le début de la phrase reflète assez fidèlement le texte, jamais il n'est question explicitement dans le texte de la joie d'échapper à une influence étrangère ou de « penser français ».

20 Est-il possible de proposer une interprétation idéologique claire de ces reformulations? Jacques Chailley est une personnalité aujourd'hui disputée à qui on reproche son action rapide dans le fichage des élèves juifs du conservatoire, comme secrétaire général, dès octobre 1940, par ailleurs très impliqué dans la vie musicale de cette époque, ce qui ne l'empêchera pas de rejoindre, semble-t-il en 1943, le Front national, mouvement clandestin de la Résistance, et de soutenir l'action de Claude Delvincourt, directeur du Conservatoire, qui sut protéger avec efficacité les élèves du conservatoire contre le STO et lutter contre les pressions de l'Occupant. Les éléments pointés montrent que Chailley n'est nullement dans un discours collaborationniste. Le fait de mentionner explicitement Ida Rubinstein n'était pas neutre, on y reviendra. Une dimension nationaliste est, par ailleurs, indéniable, mais faut-il prendre au premier degré la joie des contemporains de Jeanne d'Arc d'échapper à "l'influence étrangère des Anglais ", ou y voir, en miroir actualisé, au second degré, une image de l'occupation allemande à laquelle on rêverait de se soustraire?

\section{La réception dans la presse}

21 Dans les journaux, ce premier concert, parfois seul, parfois en lien avec l'ensemble de la semaine Honegger, a donné lieu à différents comptes rendus. L'événement étant parisien, il ne concerne qu'une presse sous contrôle allemand - et une petite partie seulement ; quand paraîtra l'enregistrement sur neuf disques de Jeanne d'Arc au bûcher, l'année suivante, on trouvera davantage d'échos dans la presse ${ }^{17}$. Notre étude focalisera son attention sur cinq articles :

- Marcel Delannoy, «La musique - La semaine Honegger », Les Nouveaux temps, 12 juillet 1942.

23 - Arthur Hoérée, «La semaine Honegger - Première et deuxième journée ", Comoedia, 4 juillet 1942, p. 5.

24 - Serge Moreux, « La musique - La semaine Honegger », La Gerbe, 30 juillet 1942, p. 8.

25 - Georges Pioch, « La musique - Festival Honegger », L'CEuvre, 1er juillet 1942, p. 2.

26 - Lucien Rebatet, «La Musique - Le festival Honegger », Je suis partout, 3 juillet 1942, p. 7.

Face à cette exécution, dans la continuité de la première réception critique de l'ouvrage antérieure à la guerre, tous les comptes rendus se révèlent louangeurs, voire 
dithyrambiques vis-à-vis du compositeur ; cela n'empêche pas néanmoins une diversité d'approches.

On peut s'amuser tout d'abord de la diversité de jugements sur la qualité de l'exécution: Georges Pioch se montre sans réserve :

M. Charles Munch, chef d'un orchestre impeccable et fervent, a prodigué à servir Honegger une puissance précise, une ardeur exaltante qui sont autant d'un démiurge que du plus enthousiaste des musiciens. Il a été parfaitement entendu par la chorale Yvonne Gouverné, la Schola de Sainte-Jeanne Chantal et les Petits Chanteurs d'Avon.

Il exprime pareillement "une louange unanime » aux différents solistes. En revanche, Arthur Hoérée évoque une " exécution insuffisamment mise au point ", ce qui ne paraitt pas étonnant, vu le programme des quatre concerts à assurer avec deux premières pour l'orchestre : L'Apocalypse de saint Jean le 11 juin et cette Jeanne qui, initialement, devait être dirigée par Honegger lui-même. Sans doute a-t-il paru plus sûr de laisser la direction de l'orchestre à son chef habituel. De son côté, Delannoy évoque les problèmes de déséquilibre entre l'orchestre et les voix: "Les récitants soutiennent contre la masse instrumentale et chorale un combat disproportionné ». Il rappelle que l'œuvre a été conçue pour l'Opéra - donc avec l'orchestre dans la fosse. Mais cela ne l'empêche pas de féliciter tous les interprètes.

On pourrait s'attendre, dans le contexte, à une valorisation idéologique de l'ouvrage qui a pour centre la figure de Jeanne d'Arc. Or les comptes rendus s'attardent très peu sur l'interprétation littéraire, voire idéologique, de l'ouvrage et sur le texte de Claudel. À titre d'exemple, Georges Pioch parle d'abord de Claudel comme «un grand poète, volontiers puéril... ", puis résume :

Les dix épisodes de Jeanne au bûcher composent un étonnant mystère dans le style médiéval. Le ciel et la terre s'y répondent : celui-là, angélique ; celui-ci, élémentaire, voire bestiale. Peuple et Foi, Prodige et candeur.

La candeur, une candeur noble, exalte le poème de M. Paul Claudel. Le prodige est surtout dans l'œuvre d'Honegger.

Marcel Delannoy consacre un petit paragraphe à Claudel dont il cite, semble-t-il de mémoire, une conférence ${ }^{18}$ :

« Jeanne d'Arc, explique Paul Claudel, m'est apparue en cet instant suprême où ceux qui vont mourir remontent en pensée le cours achevé de leur trop courte vie. » D'où ce bouleversement simple du déroulement chronologique - et ce symbolisme constant: la Cour des Bêtes présidée par Porcus (l'évêque Cauchon). Le jeu de Cartes : cette détestable politique internationale, où les rois gagnent des deux côtés. Heurtebise : le Blé du Nord. La Mère aux tonneaux : le Vin du Midi. Et le supplice lui-même devient l'Espoir, victorieux du Doute et de la Désolation.

Bien sûr, la «détestable politique internationale », dans sa formulation anachronique, renvoie à la situation contemporaine, mais sans parti pris explicite. Hoérée, de son côté, ne consacre qu'une phrase à la portée nationaliste du texte de Claudel : "On sait ce que la pastourelle de Domrémy représente pour nous et combien le mysticisme de Paul Claudel a su vivifier Jeanne, cette "réunisseuse" de "toutes les mains de la France en une seule main, une telle main qu'elle ne sera plus divisée !" ", avant de qualifier de «chef d'œuvre » l'« œuvre d'Honegger ». Quant à Lucien Rebatet, fasciste convaincu et figure engagée de la collaboration, il ne s'attarde absolument pas sur l'argument. Tout en prenant ses distances avec « ce genre assez hybride que l'on pourrait appeler l'oratorio moderne, où la récitation alterne 
avec le chant, peut se superposer avec l'orchestre ", il termine par des piques : «Dans Jeanne au bûcher, il ne faut pas moins de cinq récitants, appartenant autant que possible aux scènes subventionnées, et s'exerçant sur un texte assez abscons de Paul Claudel qui était déjà fort déclinant lorsqu'il l'écrivit, en 1934. » Outre la pique contre les théâtres subventionnés - sans doute vise-t-elle Jean Hervé au premier chef -, ce texte renvoie clairement à la haine des milieux collaborationnistes pour Claudel, le diplomate d'une Troisième République honnie, haine qui se manifestera encore davantage, dans Je suis partout, lors des représentations du Soulier de satin en 1943 que Barrault, Claudel et Honegger commençaient précisément à préparer au même moment.

Paradoxalement, la création à Chaillot en 1939, pourtant sous la même forme, avait davantage suscité des commentaires à dimension nationale. André George écrivait ainsi :

Claudel entend nous donner ici une Jeanne d'Arc préservée des Beaux-Arts et des Académies; elle ne connaît que la terre de France et le ciel - fille de Lorraine et fille de Dieu. C'est une bergère, une "Jeanne de chez nous » et qui s'épanouit dans l'héroïsme, la sainteté, le sublime parce que rien, justement, ne vient s'interposer entre la grandeur éternelle et sa divine simplicité. Les mots ont donc la saveur terrienne, le parfum des champs et l'allure des conversations vives, agrestes, que tiennent nos villageois depuis toujours ${ }^{19}$.

De même, la réception de la tournée de 1941 en zone non occupée valorisait l'œuvre " consacrée à l'idée de renaissance française authentique ", expression de "l'âme de la France en 1941, consciente de son histoire, ouverte vers l'espérance de l'avenir $»^{20}$, comme l'écrivait André Fabre dans La Croix. L'enregistrement de 1943 donnera lieu à ce même type de lecture, Robert Desnos exaltant cette " œuvre nationale ", " œuvre de circonstance, au plus beau sens du mot, qui demande à être entendue par les subtiles oreilles du cœur. $»^{21}$

Le fait que ce concert entre dans la "Semaine Honegger» focalise manifestement l'attention des critiques sur le compositeur. Un point est presque toujours interrogé qu'on peut mettre en relation avec le contexte : son inscription nationale. Honegger est de nationalité suisse, mais il est né au Havre et a presque toujours résidé à Paris. En même temps, sa formation musicale et son esthétique doivent beaucoup, comme cela a toujours été relevé, à la musique allemande. Les critiques évoquent tous cette situation. Serge Moreux, dans La Gerbe, pose directement la question mais tente de dépasser les enjeux nationaux :

Est-il Suisse ? Est-il Francais ? Il est, et cela nous suffit. Son art doit-il d'être profond à sa source germanique, d'être séduisant au climat qui l'accueille? Peu importe : son épanouissement s'explique par la chimie de tendances multiples! Arbre robuste et bien greffé, ses fruits résistent à la morsure du vent des montagnes comme à la flétrissure de celui des plaines d'île-de-France. Sa musique n'est ni agressive ni fade : elle est une synthèse.

Hoérée reprend le débat, mais l'organise en fonction des deux œuvres du programme :

Essentiellement honeggérienne par sa solide construction, son souci formel, sa force rythmique, sa frénésie contenue, elle s'oppose pourtant nettement à Jeanne au bûcher. Ici, on sent ce que le musicien né, élevé en France, doit à son pays d'adoption. Rien de plus français que ce clair langage aux sonnailles pastorales, que ces thèmes populaires non accommodés à la sauce savante du chromatique et $d u$ développement intempestif, que cette bonne humeur, où perce un peu de gouaille, cette truculence d'aujourd'hui, rien de plus latin que cette forme précise, ce tour direct qui dit ce qu'il a à dire sans réticences, ni repentir, ni introspection superflue et philosophaillante. À l'opposé, ce sont les apports alémaniques (les parents 
d'Honegger sont d'origine zurichoise) qui, selon moi, donnent à la Symphonie leur accent particulier.

Delannoy n'évoque pas cette question, qui n'apparait que de manière incidente en rassemblant les deux ouvrages : «Confusément, dans la sensibilité du public, Honegger joue le rôle wagnérien, remueur de tripes. Je persiste à voir en lui un grand musicien dramatique, très maître de l'effet ». Faut-il y lire un sous-entendu ? Là encore, c'est un lieu commun de la critique et, à l'occasion de la création à Chaillot, Gustave Samazeuilh écrivait que «Honegger est de ceux qui savent comprendre que la leçon de la Colline Sacrée, si on l'entend selon son véritable esprit, est une leçon non de sujétion mais d'indépendance ${ }^{22}$."

Rebatet reprend la question en la posant sur un plan polémique :

Il est arrivé que d'excellents nationaux me reprochent de considérer Honegger comme un compositeur honorant la musique française. Je pense que des concerts comme ceux de cette semaine devraient clore définitivement cette assez sotte querelle. Honegger, Suisse alémanique d'origine et d'ascendance, ne serait pas Honegger s'il était né à Zurich et non au Havre. Il fait le trait d'union entre la solidité, la carrure de la musique germanique et le pittoresque, la verve de la musique française. L'influence de notre culture, de notre esprit, prédominent [sic] manifestement chez lui. Ce serait être bien rabougri que de vouloir rejeter des artistes dont toute la carrière démontre justement la force d'attraction qu'a conservée notre pays.

Georges Pioch, sur ce point, est d'accord. Il salue un «musicien glorieux pour le génie français autant que pour son art, qu'il abonde à signer d'œuvres impérissables... ». Dans l'article qui annonçait les célébrations des 50 ans d'Honegger, il allait néanmoins nettement plus loin en se «réjoui[ssant] ici que l'on s'avise enfin, chez nous, de reconnaître que la musique française n'est égalée aujourd'hui par aucune autre en créateurs comme en œuvres et que, parmi ceux qui en font prospérer la primauté, Arthur Honegger est un des plus fertiles comme des plus grands ${ }^{23}$.» Cette fois, il s'agissait bien de faire de l'art musical français le vainqueur d'un conflit symbolique implicite avec la musique allemande qui, depuis l'Occupation, était très clairement mis en scène. Les nazis affirmaient en effet la suprématie de la musique germanique à l'échelle européenne et il était alors interdit, par exemple, de jouer des compositeurs français sur le territoire du Reich. Honegger, pourtant suisse, était victime de cette même interdiction, à l'inverse de ses compatriotes.

On voit donc que le contexte historique marque ces critiques tout en laissant subsister une vraie diversité. Il est cependant un point implicitement davantage chargé qui mérite l'attention. On sait que l'Occupation allemande a très vite mis en action, avec l'accord et même l'anticipation de Vichy, sa politique antisémite et ses persécutions. Le milieu musical n'y échappait naturellement pas: Darius Milhaud, compagnon du Groupe des Six, est parti aux États-Unis, et Ida Rubinstein, on l'a dit, vivait en exil à Londres. Les Concerts Colonne avaient dû être rebaptisés Concerts Pierné dès 1940. Or, le 7 juin, une des mesures les plus emblématiques de cet antisémitisme d'État était entrée en vigueur : le port de l'étoile jaune. En juillet, ce sera la rafle du Vél d'Hiv.

Dans ce contexte, un élément prenait évidemment sens : le nom de la commanditrice de l'ouvrage, Ida Rubinstein, unique interprète, on l'a vu, avant la guerre. Le fait de mentionner son nom dans le programme n'était pas sans sous-entendu. Delannoy reprend assez platement l'information: "Aussi bien, n'oublions pas que Jeanne au bûcher se présente à l'origine comme un oratorio spectaculaire, destiné aux 
représentations de Mme Ida Rubinstein ». Un article, en revanche, appuie l'information: celui de Georges Pioch. "Grâce aux libéralités hardies de Mme Ida Rubinstein, auxquelles poésie et musique doivent beaucoup, cette œuvre hautement inspirée, mystique, populaire, et, par la musique qui l'exalte, extraordinairement puissante, nous avait été révélée en 1936. » On passera sur l'erreur de date - veut-elle renvoyer de manière consciente à l'époque du Front populaire? - mais cette formulation dans le journal vigoureusement collaborationniste de Marcel Déat, fondateur du Rassemblement national populaire étonne.

Georges Pioch (1873-1953), homme de lettres à ses débuts, critique littéraire, musicologue, journaliste et homme politique, a un itinéraire assez singulier : pacifiste, membre de la SFIO puis du jeune Parti communiste, il doit quitter L'Humanité où il écrit en 1922 et se trouve exclu du parti en 1923, par opposition avec Trotski, notamment à cause de son appartenance à la Ligue des droits de l'homme. Ce pacifisme, qui le fait aussi bien condamner le fascisme que le communisme, reste sa ligne de conduite jusqu'à la guerre, ce qui ne l'empêche pas de collaborer, comme critique, à L'Euvre de Déat jusqu'en 1942 où il se retire totalement: «j'avoue mon renoncement, c'est fini, que les hommes accomplissent sans moi leur destinée ${ }^{24}$, écrit-il en 1943.

Dans ses Mémoires politiques, ouvrage paru chez Denoël en 1989, Marcel Déat écrit, parlant de son journal :

Nous retrouvions pareillement Georges Pioch, mieux disant que jamais, sonore et encore bedonnant, malgré une légère déflation, et qui allait continuer son culte de la musique. Je ne sais pas combien de fois, avant sa retraite, il fallut le défendre auprès des Allemands, sans cesse alertés par les propos énormes que le bon Pioch tonitruait à travers Paris. Il ne se passait pas de semaine, à partir de 1941, où il ne fût dénoncé comme juif ou franc-maçon. Et toujours par d'excellents confrères bien entendu. Il est rentré dans l'ombre à temps, j'espère pour prendre sans dommage le tournant historique ${ }^{25}$.

Si l'on ajoute que le commentaire de Pioch, concernant la création de la seconde symphonie, utilise les termes d'《oppression » et de "délivrance ${ }^{26}$, il semble donc aller de soi que ce commentaire de Pioch sur Ida Rubinstein contribuait à ces attaques contre lui et affirmait, au sein même d'un journal collaborationniste, un positionnement tout autre. Rien n'est simple lorsqu'on approfondit la connaissance de cette époque.

L'article de Rebatet, tout en tenant un discours inverse, conforte paradoxalement cette interprétation. Dans son article, il écrit :

Autre affaire : il n'était vraiment pas opportun de rappeler dans le programme du festival de Chaillot que Jeanne au bûcher dut le jour à une commande de la richissime et youdissime cabotine. Ida Rubinstein, et de nous évoquer du même coup ces extravagants concerts d'Orléans, où Jeanne d'Arc était célébrée par ladite Rubinstein, en présence du ministre Zay, du maire Lévy, du général Bloch.

Cela témoigne, dans le contexte de l'époque, du poids de la moindre allusion. Cela dit aussi - puisque ce n'est pas dans le programme - le souvenir sous-jacent du souvenir de la création de l'œuvre à Orléans, en 1939. À cette occasion, La France enchaînée, journal antisémite de Darquier de Pellepoix, avait édité une affiche sur laquelle on pouvait lire :

Après la conquête d'Orléans par les Juifs, celle de Jeanne d'Arc par la Juive Ida Rubinstein. [...] Le "clou" de ces réjouissances est la représentation de la Jeanne d'Arc de Paul Claudel, avec la participation de la Juive Ida Rubinstein, du franc-maçon Jean Hervé, et avec la composition musicale du Juif Arthur Honegger ${ }^{27}$. 
L'ensemble de l'article de Rebatet porte le poids de cette situation : il rappelle en effet la situation d'Honegger qui fut un des principaux compositeurs des spectacles du Front populaire :

Ceci dit, doit-on faire un grief éternel à Honegger d'avoir, avec tant d'autres, accepté des commandes du Front populaire? Pour ma part, je crois qu'en politique, une distinction est indispensable entre les gens de lettres ou d'enseignement et les artistes. Un vieux cafard démocratique comme Georges Duhamel, maints professeurs d'une judéolâtrie active sont à éliminer impitoyablement et totalement de notre vie intellectuelle. Les opinions, politiques du peintre, du musicien, du comédien n'ont par contre aucune importance lorsqu'elles ne se manifestent pas publiquement. Nous savons de reste que, tout au long de l'Histoire, les plus grands créateurs, ont servi avec une égale docilité les maitres les plus divers. Ce qu'ils demandent avant tout à un régime, c'est de leur permettre de travailler.

Si nous avons vu en France tant d'excellents artistes incliner assez naïvement vers l'extrémisme marxiste, il faut bien dire que la faute en est d'abord à la République modérée, à la bourgeoisie conservatrice, qui n'ont cessé de manifester les goûts les plus détestables, qui se sont toujours détournées stupidement de ce qui était neuf et hardi. Fasciste cent pour cent, je rêve d'un régime où l'autorité servirait, après avoir d'abord déjudaïsé à fond les arts, à distinguer, à encourager les vrais talents. Il ne devrait plus y avoir, pour les artistes, d'autre dogme politique que celui-ci : « À bas les bourgeois! » Le jour où un ministre des Beaux-Arts poussera chez nous ce cri avec eux, la France redeviendra une nouvelle Athènes.

Rebatet, condamné à mort à la Libération, sera gracié et restera emprisonné jusqu'en 1952.

Le titre de cet article portait une question : « une simple reprise? » La réponse est, bien évidemment, non.

Le regard qu'on porte aujourd'hui sur la vie artistique qui s'est poursuivie pendant toute cette période est, généralement, critique. On donne raison à Jean Guéhenno, affirmant dans son Journal des années noires :

Il fallait que Paris fût éteint, puisque les traîtres et les ennemis l'avaient éteint. Il fallait que le monde entier sentît qu'à la place où brillait d'ordinaire tant de lumière il n'y avait plus qu'un grand trou noir, d'où ne jaillissait plus aucune parole, aucune pensée, et que ce trou noir fît honte au monde ${ }^{28}$.

Or, on le sait, la vie artistique pendant cette période fut au contraire très riche, attirant un large public particulièrement avide d'art, sans doute pour tenter d'échapper à une existence étouffante marquée par les privations, mais aussi pour tenter d'espérer et de trouver des raisons de vivre. Une œuvre comme Jeanne d'Arc au bûcher participait de cette expérience. À l'instar de la dernière réplique du Soulier de satin, «Délivrance aux âmes captives ", que la Comédie-Française fera résonner l'année suivante, la fin de l'ouvrage offrait une sublimation dont les auditeurs étaient avides. Comme le notait Chailley dans le programme, $\mathrm{y}$ « retentit une dernière fois le chant du rossignol, qui, au début de l'œuvre, opposait dans les voix-mêmes de la nature les forces de l'Espoir aux hurlements de la Désespérance vaincue ».

53 On est surtout sensible aujourd'hui à la compromission que manifeste cette vie artistique parisienne avec l'occupant qui la contrôle et en profite largement. Que vaut d'ailleurs cette audition alors que la rafle du Vél d'Hiv est en préparation? Les articles évoqués montrent cependant que le contexte est présent de manière sous-jacente. Selon des degrés divers, l'œuvre est toujours mise en relation avec les années trente et avec sa mécène et interprète. On est sans doute loin d'actes de Résistance, mais la 
réaction de Rebatet montre que cette attitude était sans aucun doute volontaire, y compris dans le cas de Marcel Delannoy qui, membre du Groupe Collaboration et critique aux Nouveaux Temps de Jean Luchaire, aura à rendre des comptes à la Libération.

En août 1944, alors que les combats pour la Libération de la France s'intensifiaient, Claudel a rédigé un prologue pour Jeanne d'Arc au bûcher faisant explicitement résonner l'ouvrage dans son lien avec l'actualité par le biais de citations bibliques. Il était prévu des représentations de gala à l'Opéra, mais elles ne purent avoir lieu, la musique d'Honegger se trouvant alors exclue des programmes. On reprochait notamment à Honegger d'avoir fait le voyage à Vienne en 1941 pour le $150^{\mathrm{e}}$ anniversaire de la mort de Mozart et d'avoir été trop visible et trop joué en cette période. 1945 marque cependant un coup d'arrêt, très temporaire: Ida Rubinstein redonne l'œuvre à Strasbourg en 1946 et 1947 pour ses dernières apparitions sur scène; le chef d'orchestre Louis de Vocht la dirige à Paris, après Bruxelles, en 1947 et sa renommée s'étend très vite au monde entier où elle fait toujours partie des rares œuvres lyriques $\mathrm{du} \mathrm{Xx}^{\mathrm{e}}$ siècle à être jouées. Cette reprise de Jeanne d'Arc au bûcher à Chaillot en 1942 peut paraître appartenir à une face sombre de son histoire, mais l'examen attentif des documents montre aussi que cette audition était bien saisie dans le souvenir et la continuité d'une époque passée contre le moment présent, comme un signe d'espérance aussi, loin de toute compromission avec l'occupant.

\section{NOTES}

1. Voir la reproduction d'une photographie de ce rassemblement dans Colette Godard, Chaillot. Histoire d'un théâtre populaire, Paris, Le Seuil, 2000, p. 18.

2. GODARD (Colette), Chaillot, Un théâtre national et populaire, Paris, Norma Éditions, 1998, p. 15.

3. Voir ORY (Pascal), Le Palais de Chaillot, p. 96 et GODARD (Colette), Chaillot, Un théâtre national..., op. cit., p. 19. Karine Le Bail, dans La Musique au pas. Être musicien sous l'Occupation, Paris, CNRS Éditions, 2016, évoque "presque 3300 spectateurs", p. 42, ce qui permet de grossir les foules se pressant aux concerts collaborationnistes. Alan Riding, dans Et la fête continue: La vie culturelle à Paris sous l'Occupation Paris, Plon, 2012, p. 189, avance le chiffre de 3500 places.

4. Voir http://www.ina.fr/video/AFE85000549

5. Voir LE BAIL (Karine), op. cit., p. 42.

6. Cet article revient, de manière plus restreinte et plus précise, sur une étude de réception générale qui a fait l'objet d'un article, "La réception de Jeanne d'Arc au bûcher de 1940 à 1945 ", écrit en 2003 et publié dans LÉCROART (Pascal) dir., Claudel politique, Lons-le-Saunier, Aéropage, «Penser!», 2009. Les nombreux travaux publiés depuis sur cette époque ont, en partie, fait évoluer mon point de vue.

7. Pour une approche générale de cet ouvrage, voir CALMEL (Huguette) et LÉCROART (Pascal), Jeanne d'Arc au bûcher de Paul Claudel et Arthur Honegger, Genève, Éditions Papillon, « $7^{\mathrm{e}}$ note ", 2004.

8. Sur Ida Rubinstein, à qui l'on doit Le Martyre de saint Sébastien de Gabriele d'Annunzio et Claude Debussy, ou le Boléro de Ravel, voir DEPAULIs (Jacques), Ida Rubinstein. Une inconnue jadis célèbre, Paris, Champion, « Dimension », 1995. 
9. Voir CALMEl (Huguette) et LÉCROART (Pascal), op. cit., p. 28-34.

10. Chaque couple était chargé d'un épisode: Maurice Fombeure et Louis Beydts pour l'enfance, Jean de Beer et Georges Dandelot pour l'épisode de Vaucouleurs, Raymond Loucheur et José Bruyr pour la rencontre avec le dauphin, Exbrayat et Tony Aubin pour Orléans, Henri Ghéon et Jacques Chailley pour le sacre, Gilles Gérard et Pierre Capdevielle pour le procès, Claude Vermorel et André Jolivet pour le bûcher.

11. BARBARIN (Maurice), « “Jeanne d'Arc au bûcher" à Lyon ", L'Action française, 7 août 1941, p. 4.

12. Voir LE BAIL (Karine), op cit., p. 211-212.

13. Ce programme, consulté à partir d'une collection privée, se présente sur une simple feuille en format A3 pliée en deux. On peut légitimement penser que le contenu était identique à celui du concert du soir.

14. "Un comité de propagande pour la musique », Le Figaro, 21 janvier 1931, p. 5.

15. Sur les Jeunesses musicales de France dans le cadre de l'Occupation, voir simon (Yannick), «Les Jeunesses musicales de France », dans CHIMÈNEs (Myriam) dir., La Vie musicale sous Vichy, Éditions complexe, 2001, p. 203-215.

16. Voir CHIMÈNEs (Myriam) et SIMON (Yannick) dir., La Musique à Paris sous l'Occupation, Paris, Fayard/Cité de la musique, note 1, p. 21.

17. Voir notamment le dossier de presse (1940-1943) établi par la BnF et consultable à l'adresse : https://catalogue.bnf.fr/ark:/12148/cb42674163c.

18. Dès 1935, Claudel a rédigé le texte d'une conférence sur Jeanne d'Arc au bûcher, publiée dans Conferencia, le $1^{\mathrm{er}}$ octobre 1935. Par la suite, il a repris ce texte pour des conférences avant les auditions de l'oratorio dramatique, notamment à Bâle et à Orléans. Ce n'était pas le cas en 1942. La citation de Delannoy ne se retrouve pas à l'identique dans la version originale de la conférence.

19. GEORGE (André), « Jeanne au bûcher », Les Nouvelles littéraires, 17 juin 1939.

20. FABRE (André), "Jeanne d'Arc au bûcher de Paul Claudel et Arthur Honegger ", La Croix, 13-14 juillet 1941.

21. DESNOS (Robert), « Disques - Jeanne d'Arc au bûcher », Aujourd'hui, 21 juillet 1943.

22. SAMAZEUilH (Gustave), «La musique - Jeanne au bûcher de P. Claudel et A. Honegger au Palais de Chaillot », Les Annales politiques et littéraires, 25 juin 1939, p. 633.

23. Ріосн (Georges), «Un grand musicien français - Les 50 ans d'Arthur Honegger », L'Euvre, 26 juin 1942.

24. Lettre à Jeanne Hubert du 28 septembre 1943, citée dans la notice Georges Pioch, rédigée par Nicolas Offenstadt, du Dictionnaire biographique du mouvement ouvrier français, Jean Maitron (dir.), accessible à l'adresse : http://maitron-en-ligne.univ-paris1.fr/spip.php?article24396.

25. DÉAT (Marcel), Mémoires politiques, Paris, Denoël, 1989, p. 566.

26. «Cette symphonie est tout un drame. Dramatique, le premier mouvement, obsédant, lancinant par la mélancolie de sa plainte obstinément répétée. Dramatique aussi, l'adagio, dont la douleur tourmentée s'apaise peu à peu, pour se rasséréner dans une cantilène expressive de paix. Dramatique enfin, le troisième mouvement, qui libère magnifiquement la symphonie de l'oppression où elle se débattait. L'irruption des trompettes sonne la délivrance. Dès lors, et jusqu'à la fin, c'est une liesse de l'homme jeune, sur laquelle plane, éclatant, cordial, irrésistible, un choral où Honegger témoigne pour sa filiation à Jean-Sébastien Bach. Mais celui-ci n'est-il pas l'alpe où prennent leur source et de laquelle abondent les plus grands fleuves de la musique ?»

27. Texte cité par Michel Winock, «Jeanne d'Arc», dans NORA (Pierre) dir., Les Lieux de mémoire, t. 3, Paris, Gallimard, « Quarto », 1997, p. 4464.

28. GUÉHENNo (Jean), Journal des années noires, Paris, Gallimard, « Folio », 2002 [1947], p. 10. 


\section{RÉSUMÉS}

Le 13 juin 1939, le Palais de Chaillot accueille la création parisienne de Jeanne d'Arc au bûcher, oratorio dramatique de Paul Claudel et Arthur Honegger commandé par Ida Rubinstein qui interprète le rôle-titre. Trois ans plus tard, le 25 juin 1942, l'œuvre est reprise dans le même lieu, avec le même incontestable succès, mais dans un tout autre contexte. À partir de la réception critique dans la presse et de documents d'époque, l'article s'interrogera sur les interprétations différentes qu'on est susceptible de construire et de reconstruire de l'œuvre et de son exécution, son autonomie esthétique étant naturellement contestée par les significations idéologiques sousjacentes, fondamentalement ambiguës, portées par les deux époques, si proches et si lointaines.

INDEX

Mots-clés : Palais de Chaillot, Gémier (Firmin), Aldebert (Pierre), Jeanne d'Arc au bûcher, Claudel (Paul), Honegger (Arthur), Rubinstein (Ida)

\section{AUTEUR}

PASCAL LÉCROART

Professeur, université de Franche-Comté, membre du projet ANR ECHO 


\title{
Aller à Chaillot : des spectateurs d'aujourd'hui parlent et se souviennent
}

\author{
Hélène Bouvier
}

1 Le Théâtre de Chaillot constitue l'un des lieux d'étude privilégié du projet ANR ECHO [ECrire l'Histoire de l'Oral. L'émergence d'une oralité et d'une auralité modernes. Mouvements du phonique dans l'image scénique (1950-2000)], consacré à l'étude de l'oralité et de l'auralité du théâtre français de la seconde moitié du $\mathrm{xx}^{\mathrm{e}}$ siècle. Chargée du volet de recherche portant sur la mémoire des spectateurs, c'est l'animation de ces souvenirs livrés et partagés lors de plus d'une vingtaine d'entretiens depuis 2014 que je vais tenter de transcrire, au plus près des mots qui les ont exprimés, tout en déplaçant le focus vers ce lieu unique de Chaillot et sans y appliquer le filtre de l'approche phonique, sonore ou acoustique, initialement choisi pour le programme ECHO.

2 En questionnant l'adoption ou le rejet d'un lieu, la fidélité à ce lieu ou son abandon, c'est, au-delà, ce qui mobilise littéralement un spectateur de théâtre dont il est question : tenter de saisir, finalement, pourquoi plusieurs de ces témoins ont fait le déplacement pour les journées d'étude de juin 2018 consacrées à Chaillot, mus à nouveau par leur relation particulière à ce lieu ${ }^{1}$.

3 La relation est à la fois personnelle, puisqu'elle suit le fil de vie de chacun et ses aléas, collective puisque partagée le temps d'une représentation tout en perdurant bien audelà, et évolutive au gré des directions successives, des transformations architecturales du lieu ou de sa réorientation, mais aussi des goûts et des pratiques du spectateur vers d'autres arts vivants comme la danse, par exemple.

Ces spectateurs d'aujourd'hui parlent et se souviennent :

5 d'un lieu qui a changé depuis, et plus précisément de la grande salle de chaillot ;

6 d'une direction et d'une troupe parmi tant d'autres là ou ailleurs, principalement celle du TNP de Jean Vilar, puis de Georges Wilson;

7 du théâtre parmi les autres arts vivants qu'ils fréquentent ou pratiquent aussi, comme la danse ou la musique, qui seront seulement évoqués à la fin de cette étude. 


\section{Un lieu et une architecture, une empreinte et une emprise}

8 À la question, "qu'est-ce qui vous revient en premier de Chaillot? ", c'est ce lieu physique investi par ses hôtes, dans le double sens du français, de ceux qui accueillent (le directeur et sa troupe) et de ceux qui sont accueillis (les spectateurs), qui surgit d'abord.

Ainsi : «En premier, le souvenir de la salle, en deuxième, des pièces que je découvrais, Brecht, Sartre, une ouverture extraordinaire, un souvenir très fort, une sorte de célébration, avec joie et sérieux ». Ou encore, avec le rappel des matinées scolaires: «D'abord une ambiance, quand vous arriviez à l'époque sur la place du Trocadéro, on avait l'impression qu'il y avait une manifestation, tellement il y avait de monde! Des jeunes, il y en avait partout, c'était très très animé et c'était une image qui m'a dès le départ énormément surprise de voir cette foule, et en fait effectivement, la salle était très grande, elle tenait au moins 2000 places ».

10 Tous les spectateurs parlent d'emblée d'un lieu immense qui les a impressionnés, qui englobe dans un continuum hall, salle et scène, si différent des théâtres alors habituels, et presque tous mentionnent l'escalier d'accès : « ce qui revient à l'esprit c'est le hall. Je suis toujours impressionné par cette volée de marches. C'est très différent des théâtres à l'italienne ou théâtres de boulevard, tout riquiquis : je suis impressionné par le lieu »; " un escalier monumental»; un " escalier qui n'en finissait plus, une grande salle avec des vitres, très ample, sans arc de cercle »; «l'impression de rentrer dans une boîte », "seuil, salle immense, scène en bas, grand volume ", avec le souvenir d'un «très très grand plateau ", « un plateau très ouvert avant Vitez ».

11 La jauge de la salle, de plus de 2000 places, n'est pas ressentie comme une gêne, si ce n'est une sensation de lointain parfois évoquée dans un «souvenir de foule, d'espace, de lointain [...] je me sentais toujours trop loin, un souvenir d'espace, l'envie de m'approcher "; " quand on était au balcon, on était vraiment loin mais il n'y avait pas de problème pour entendre, ils devaient parler fort et avoir une très bonne diction ».

Une spectatrice englobe sa perception et son expérience de ce lieu jusqu'à nos jours : « il fallait descendre toutes ces marches, on descendait comme dans une crypte, comme dans une cathédrale. Pas du tout comme maintenant comme on va n'importe où : il y avait une espèce de gravité. C'était une vraie sortie. On s'attendait à quelque chose, il y avait vraiment une atmosphère dans cette immense salle du TNP avant les travaux ».

Justement ces travaux, tous ceux qui ont connu les lieux avant les transformations de 1973 en ont le regret: "c'était un beau lieu»; "je reste inconsolable de la transformation de la salle [...] un lieu très contraignant où on était excessivement heureux »; «je ne suis retournée à Chaillot qu'en 1994, je me suis dit 'formidable, ça fait tellement longtemps que je n'y suis pas allée', cet escalier, formidable et tout, et quand je suis rentrée dans la salle, horreur! J'avais gardé le souvenir de cette salle immense, très large, et là je me retrouve dans une salle que je trouve toute riquiqui, toute moche, toute rouge. Alors ça, ça a été le choc. Je me suis dit, c'est un théâtre ordinaire ». 
Le lieu est parfois qualifié de "froid et peu accueillant", comparé à "d'autres salles plus chaleureuses, comme Renaud-Barrault », mais dans ce cas il s'agit de la salle transformée.

Enfin, un autre élément constitutif de cette perception et de cette mémoire doit être pris en compte : l'hôte de ces lieux qui voient se succéder, pour cette période d'avant les travaux, Jean Vilar puis Georges Wilson.

\section{Direction, troupe, répertoire et scénographie : une cohérence perçue et remémorée}

6 Les choix de répertoire faits par la direction du théâtre sont clairement perçus par les spectateurs. Pour la période Vilar, en plus des pièces du répertoire classique, ils ont été marqués par celles choisies pour leur actualité sociale et politique, leur résonance avec la société ou les vertus humaines, un répertoire servi par le jeu des acteurs pour une "découverte ", une " ouverture sur un monde que je ne connaissais pas ", une "prise de conscience ", une "révélation de pièces et de mises en scène " selon les mots de chacun. Une spectatrice déclare avec enthousiasme : «j'étais motivée par les thèmes, les idées ». Plusieurs exemples de pièces restent vivaces. Ainsi : «Dans La Guerre de Troie n'aura pas lieu, le discours aux morts dit par Pierre Vaneck et les imprécations de Cassandre par Judith Magre, ça fait réfléchir à 14 ans »; " et aussi L'Alcade de Zalaméa, alors que les problèmes politiques débordaient la classe politique [...] comme quand il a joué Thomas More; la justice, l'équité sont des vertus humaines qu'il ne faut pas bousculer sinon, c'est une société qui en meurt, c'est actuel, quotidien, sincère ».

Le contexte de la guerre d'Algérie surgit de plusieurs façons : «avec Arturo Ui pendant la guerre d'Algérie et la crainte d'une montée fasciste, le public vibrait», ou «le message politique dans Antigone de Sophocle ou Arturo Ui, pièces où Vilar dit 'non' ». Après Vilar, un spectateur a le souvenir vivace d'un « Nicomède avec Laurent Terzieff, en pleine guerre d'Algérie, la mise en question du colonialisme romain ».

8 La modernité du propos est aussi évoquée et mise en relation avec la jeunesse de la troupe : « une pièce comme L'Agression, en 1967, le début du problème de la société de consommation, c'était donc très moderne, dans l'air du temps, prémonitoire, une pièce très très engagée. Une troupe avec des comédiens qui donnaient une impression de grande jeunesse ».

Sur un autre thème, «Le Diable et le Bon Dieu, de Sartre, ça compte », le continuum entre texte, jeu, perception et compréhension est remémoré comme un "extraordinaire débat, je me souviens de la salle, des acteurs, de l'autorité qui se dégageait : un moment où on devient intelligent, une ouverture extraordinaire ». Ce dernier mot revient pour la période Wilson, «l'ouverture d'esprit, un lieu qui nourrissait, avec Maître Puntila et son valet, En attendant Godot, Le Cercle de craie caucasien: des pièces modernes ». Et cet ancrage autobiographique souvent vérifié: «j'étais jeune, donc tout ce qui était moderne, c'était plus facile, contemporain ».

Au-delà de la modernité, les spectateurs du TNP soulignent la nouveauté du répertoire par rapport à d'autres théâtres, tout en les associant à des contextes sociaux contrastés, notamment avec la Comédie-Française et le théâtre de boulevard. «Le TNP c'était l'avenir, c'était demain, c'était aujourd'hui, et le Français c'était le passé, les classiques, la famille [...] Partout, sur scène et dans la salle, c'est deux mondes différents, et ça, 
c'est sociopolitique »; "c'était différent de la Comédie-Française d'une classe plus élevée "; "Le TNP et le théâtre de boulevard, je voyais bien que ce n'était pas pareil, deux expériences complètement opposées. Moi j'avais l'impression que Chaillot c'était le théâtre du peuple, le Palais que l'on avait offert au peuple, c'était magnifique, c'était des grands espaces, c'était ce grand escalier et en même temps, c'était populaire [...] On était chez soi, chez soi dans un Palais et on allait voir des œuvres, il y avait quand même un petit côté d'éducation, d'ouverture, on allait comprendre des choses ".

Quant à la scénographie de cette période TNP, et en particulier celle de Vilar, tous ses éléments - mise en scène, décors, costumes, lumières - suivent une même ligne directrice: "dénuement, efficacité, sans fioriture avec pourtant la capacité de mouvements de foule sur le plateau, et d'enthousiasmer le public ». On se remémore une " lumière travaillée, qui définit des groupes, des mouvements, lumière de l'ombre, du noir et blanc, noir et marron, pas jaune plate partout comme celle du théâtre de boulevard", un "théâtre contemporain avec des mises en scène différentes de la Comédie-Française, beaucoup plus dépouillées », un " décor minimum, une pureté, une sobriété des costumes, un éclairage proche des visages des acteurs", avec des " costumes qui comptaient beaucoup, des scènes en clair-obscur, des effets de lumières, parfois sombres ", "ce qui était frappant, c'était les costumes et les lumières ", ou encore « les costumes, on les voyait». Et ce constat unanime : «les décors et costumes synthétiques de Gischia, l'unité de style de Vilar, rompue ensuite ».

Un spectateur se souvient avoir vu deux spectacles dans la grande salle de chaillot d'avant Vilar, sous la direction de Pierre Aldebert, qui réduisait alors le cadre pour réaliser ses décors, tout en laissant une impression de salle glaciale. En revanche, " Vilar, c'était saisissant: une utilisation de la scène en pleine largeur, souvent sans rideau de scène. [...] un grand espace noir, une absence quasi totale d'accessoires. [...] La carriole de Mère Courage dans cette immensité, ça avait une sacrée allure. Ce n'était pas un jouet! Il y avait de la grandeur dans tout ça, et de la grandeur familière ». Il a ainsi le souvenir vivace de «Vilar tout seul, dans sa prison, assis sur son tabouret avec juste une cruche à côté, dans l'immensité noire : ces moments de monologue dans cette salle immense étaient des moments-clé des spectacles. Et ça, ça a toujours eu une force extraordinaire qui était due au rapport de 1 pour 2000. Un rapport d'échelle. Et une certaine grandeur aussi dans le fait qu'il y ait un protagoniste seul face à lui-même, aux dieux et au public ».

\section{Les effets conjugués de l'accueil du public et de la programmation du TNP}

Les entretiens menés de manière ouverte, peu dirigée ${ }^{2}$, ont laissé l'occasion aux spectateurs de se remémorer des éléments souvent négligés ou considérés comme périphériques à l'expérience strictement théâtrale: presque tous se souviennent de l'accueil du public au TNP et de l'atmosphère qui régnait dans la salle, et en tout premier lieu, $\mathrm{du}$ «bouleversement d'habitude considérable avec Vilar concernant les horaires, les tarifs ». "On voyait: 'pourboires interdits', et 'vestiaires gratuits' »; ou encore : «le théâtre, c'est un monde un peu luxueux, et là, une expérience de théâtre différente, abordable». Expérience que l'on veut goûter le plus longtemps possible: «j'arrivais avant l'ouverture des portes pour voir la salle se remplir», et qui est 
partagée en foule : « les salles étaient pleines, je me souviens de plein plein de monde qui descendait, et au départ, pareil ».

Les témoignages mêlent ce souvenir d'un accueil inhabituel, d'une attente et même d'une anticipation partagée, avec une qualité d'attention, de concentration, allant jusqu'à une «adhésion » ou même une « communion ». On se souvient de la «foule des matinées scolaires, silencieuse, écoutant, une attention tendue, une attention haletante, désirante, pas de papiers froissés, de sièges qui grincent", de ce public " réceptif, discipliné » dont convient facilement aussi ce spectateur, même s'il fut un jour " témoin d'un chahut scolaire avec Gérard Philipe et Maria Casarès dans Le Cid ". On entend encore le «brouhaha de la descente des escaliers, dehors, et dès que le spectacle commençait, il y avait un silence religieux, il n'y avait pas de papiers de bonbons comme maintenant ", et "même quand on était des camarades, on n'aurait pas chuchoté, on n'aurait rien dit ». Et pourtant, un " public extrêmement chaleureux, qui applaudissait ». Une spectatrice exprime ainsi avoir « toujours senti la présence des autres, la communion au TNP, assez profonde ».

En cherchant les mots pour qualifier ce lieu si particulier et qui a laissé des traces durables chez les spectateurs de cette période, Chaillot est ainsi appelé « le temple de la connaissance " ou «la maison de la connaissance moderne». On explique que «ce n'était pas une sortie de spectacle, c'était un émerveillement", ou encore "une somptuosité démocratique». Ce dernier registre est souvent évoqué, comme cette spectatrice de théâtre chevronnée qui déclare avoir «beaucoup aimé le TNP parce que c'était vaste, libre, une première impression aussi sensible d'un palais élevé pour le théâtre. Ce qu'il y avait de mieux, avec un plateau très dégagé pour voir dans les meilleures conditions, et aussi pour l'acteur, sinon il n'a pas sa liberté. On se sentait à sa place, comme public, pas le côté guindé de s'habiller, etc. ». Un spectateur déplie ainsi son expérience de son entrée à sa sortie de la grande salle : c'était "comme si on rentrait dans un temple, dans un bâtiment de la culture [...], le fait de rentrer dans la salle, je n'étais plus la même personne, je changeais de peau. Et quand j'en sortais aussi, la rue, je la trouvais laide, décevante, tout à coup je voyais cette agitation oh combien inutile! Après le spectacle, je sentais une agitation inutile; une fois que je me trouvais sur le parvis du Trocadéro tout cela me paraissait bien misérable [...] Quand on sortait du TNP, on était différent : on sentait qu'on avait assisté à un don... L'après n'était plus du tout comme l'avant. Et cela m'a d'autant plus touché que l'architecture était massive, sans fioritures, et c'était le théâtre de la parole. On allait plus à l'essentiel, en tout cas l'essentiel apparaissait, quelque chose de très fort " .

Des qualités humaines sont même attribuées à ce lieu physique : «c'était un lieu pas mesquin, petit, étriqué, on peut y aller, c'est flamboyant », « l'allégorie, la générosité, le grandiose, et je pense qu'un acteur comme Gérard Philipe le comprenait à merveille ", " ça respirait large, il n'y a qu'à voir la différence entre TNP et Comédie-Française pour Le Cid, par exemple». Finalement, ce lieu était littéralement animé, il «avait du souffle » selon l'expression de plusieurs spectateurs.

\section{Une expérience personnelle indélébile}

" Ça a marqué des âmes fraîches, les âmes de l'adolescence ", remarque justement une spectatrice. "Découvrir Brecht à 10 ans, c'est violent! J'avais pressenti que cette enfance était un peu protégée, c'était une phase de la vie, mais ce n'était pas la vie, 
donc la confrontation avec ce que je ne connais pas, avec Mère Courage et Montero, c'est mon premier rapport avec la violence et : ' il n'y a pas que des choses agréables dans la vie' ", analyse rétrospectivement un spectateur. Pour certains, en effet, " aller au TNP c'était sortir du carcan", mais à l'inverse, pour d'autres, "c'était retrouver ce que j'entendais à la maison ", à la différence de ce qui se jouait dans d'autres salles. Une spectatrice associe sa "découverte du théâtre avec le TNP de Vilar, à 15 ans. J'ai été frappée par l'émotion qui se dégageait du spectacle d'Antigone de Sophocle ", et une autre englobe sa passion de toute une vie pour le théâtre dans ces mots : " ma grande entrée dans le théâtre, c'est le TNP ».

Le caractère unique et indélébile de cette expérience est réitéré au fil des entretiens, de manière unanime, mais diversement exprimée dans le parcours de chacun : « le TNP ça me reste comme si c'était hier »; «c'était pas juste une soirée et on oublie. Ce souvenir de théatre est à part, il n'a pas de lien avec la suite [de mon expérience de spectatrice de théâtre]»; "Vilar, suivi par Wilson, l'ambiance d'une troupe bien soudée, bien dirigée, après, je ne suis plus jamais retournée [à Chaillot] »; «j'ai cherché des épigones de Vilar en banlieue, puis j'ai basculé sur la danse ».

En effet, d'autres expériences artistiques à Chaillot sont incidemment rappelées, en dehors du théâtre, comme la musique et la danse. Un spectateur rappelle notamment que la grande salle était "aussi salle de concert, avec l'orchestre Pasdeloup». Une spectatrice se souvient « des concerts gratuits pour les abonnés de Bref, pour les 10 ans du TNP », une autre indique : «j'allais aussi aux concerts du dimanche soir ». Plusieurs spectateurs ont continué d'aller à Chaillot une fois sa mission principale affichée sur la danse, une personne décrivant ainsi être « allée récemment à des fêtes de la danse. Il y avait cinq ou six chorégraphes qui occupaient chacun une salle parce qu'il y a beaucoup de petites salles et même d'autres espaces, et qui vous faisaient danser. Et c'était très intéressant, parce que c'était des chorégraphes de cultures très différentes et d'approches du corps très différentes. Ça amenait à une compréhension de la danse contemporaine très différente de [celle qu'on peut avoir seulement] quand on regarde ». Fidèle au lieu, un autre spectateur confie en souriant «j'y vais maintenant pour la danse ; je suis toujours ébahi par la Tour Eiffel ».

\section{Conclusion}

Deux pôles paraissent habiter les souvenirs des spectateurs de la grande salle de Chaillot, l'un tendant vers le cérémonial, l'intangible, et l'autre vers le moderne, le contemporain. Il me semble qu'ils renvoient précisément à l'architecture du lieu et aux choix d'une direction et de sa troupe concernant le répertoire, la scénographie et les conditions d'accueil du public.

31 Les spectateurs parlent aussi explicitement de la dualité entre l'individu et le collectif sur cette période particulière, pour le théâtre et dans leur parcours de vie. L'étonnement initial peut marquer, accompagner ou même déterminer une vie. L'adhésion est emportée par la perception d'un travail de troupe attaché à ce lieu précis, sans que l'on sache toujours l'éclaircir. Dans cette quête de compréhension et d'analyse réflexive, les personnes les plus âgées peuvent dire qu'« il y avait un grand sérieux, c'était l'idée qu'on allait au TNP : est-ce que c'était le respect que tout le monde avait pour Vilar, ou bien mon âge, beaucoup plus jeune, beaucoup plus sensible? »; ou bien «Chaillot, la plus belle partie de mon histoire de spectatrice de théâtre », ou de 
manière encore plus explicite :«je suis la génération du TNP, c'est une expérience globale ».

De nombreux spectateurs de la période du TNP ont fait le pas du théâtre vers la danse, si l'on peut dire. Une personne résume son parcours "Vilar au TNP puis Béjart découvert à Avignon en 1966, [que j'ai] suivi ensuite ailleurs. Le choc de la danse, Béjart c'était du théâtre ». Pour une autre : «je suis ressortie changée » après la découverte de Béjart, expérience parfois précisée ainsi : «il y a eu aussi Béjart avec sa Messe pour un temps présent ».

Dans ce glissement du théâtre vers la danse, s'agit-il d'un simple changement de goût ou de l'adaptabilité des spectateurs aux contextes changeants, ou encore d'une perméabilité entre cette pratique théâtrale et cette danse contemporaine qui émerge? Certes, on peut rappeler à ce sujet la proximité entre Vilar et Béjart, et la concomitance des bouleversements en théâtre et en danse qu'ils ont portés. À ces éléments factuels, il faut ajouter, pour notre propos, la transformation de l'architecture d'un lieu qu'on avait adopté, la disparition du maître de maison, Jean Vilar, et enfin le déplacement de ces pratiques théâtrales vers d'autres lieux, accompagnant l'évolution de la mission de Chaillot vers la danse. Ainsi s'agrègent finalement beaucoup de raisons pour reconnaître l'empreinte indélébile d'« être allé à Chaillot », il y a parfois longtemps, parmi les incessantes découvertes liées à la passion d'être spectateur.

\section{BIBLIOGRAPHIE}

La Matière des émotions. Les arts du temps et du spectacle dans la société madouraise (Indonésie), Paris, École française d'Extrême-Orient, 1994.

Theatre Research International. Special Issue: Anthropology and Theatre (éd. invité), vol. 19, $\mathrm{n}^{\circ} 1$, Oxford University Press, 1994.

Théâtres d'Asie à l'œuvre. Circulation, expression, politique, avec Gérard Toffin (dir.), Paris, École française d'Extrême-Orient, 2012.

L'Art du pathétique en Asie du Sud-Est insulaire. Le choix des larmes, (dir.), Paris, L'Harmattan, 2013.

L'Écho du théâtre. Dynamiques et construction de la mémoire phonique, $\mathrm{XIX}^{e}-\mathrm{XX}{ }^{e}$ siècle, avec Marion Chénetier-Alev (dir.), Revue Sciences/Lettres [En ligne], n 5/2017, Éditions Rue d'Ulm, mis en ligne le 2 octobre 2017, http://rsl.revues.org.

\section{NOTES}

1. Je tiens à remercier chaleureusement pour leur temps et leur confiance toutes les personnes qui ont accepté de partager leur mémoire de spectateur au cours de ces entretiens, moteurs enthousiastes de cette recherche au long cours. En dehors de ceux qui ont souhaité rester anonymes, ces "coauteurs" de mon étude sont Isabelle Bloch, Marie-Louise Bloch, Simone Drouin, Geneviève Dubois-Kervran, Françoise Gondal, Lionel Héligoin ( $\dagger$ ), Françoise Hubert- 
Guillou, Jacques Kraemer, Patrick Liegibel, Pierrette Lombès, Christian Lot, Maud Rayer, Annick Roussel, Jean Roux, Yves Sartiaux, Catherine Sfez, Françoise Viatte, Frédérique Victoire, JeanMarie Villégier.

2. Je me permets de renvoyer à deux de mes articles portant sur la méthodologie et les conditions de ces entretiens: "Expérience auditive et mémoire phonique dans les entretiens avec des spectateurs de théâtre ", dans L'Écho du théâtre, Revue Sciences/Lettres [en ligne], 5/2017, Éditions Rue d'Ulm, mis en ligne le 2 octobre 2017, http://rsl.revues.org/1200, et «De l'usage expérimental des archives audio dans la recherche sur la mémoire des spectateurs ", L'Écho du théâtre 2, Revue Sciences/Lettres [En ligne], 6|2019, Éditions Rue d'Ulm, mis en ligne le 10 décembre 2018. URL : http://journals.openedition.org/rsl/1743.

\section{RÉSUMÉS}

Aller au spectacle, au théâtre, au TNP, à Chaillot : autant de manières d'exprimer la relation entre un spectateur et un lieu particulier, unique puisqu'indissociable des aléas de chaque vie, et pourtant ressentie comme collective, dans le temps d'une représentation et au fil des directions et des transformations, de la grande salle en particulier. Une vingtaine d'entretiens fonde l'animation de ces mémoires qui remontent jusqu'à Vilar, questionnant l'adoption, le rejet ou l'abandon d'un lieu, et au-delà, ce qui mobilise littéralement un spectateur de théâtre.

\section{INDEX}

Mots-clés : Théâtre national populaire, Théâtre national de Chaillot, Vilar (Jean), mémoire théâtrale, mémoire du spectateur, entretiens

\section{AUTEUR}

\section{HÉLĖNE BOUVIER}

Directeur de recherche au CNRS, anthropologue, HDR en études théâtrales, unité mixte de recherche THALIM « Théorie et histoire des arts et des littératures de la modernité $\mathrm{XIX}^{\mathrm{e}}-\mathrm{XXI}^{\mathrm{e}}$ siècle » (UMR7172, CNRS, École normale supérieure, université Sorbonne nouvelle-Paris 3, membre du projet ANR ECHO 


\title{
Béjart à Chaillot : une anti-histoire de la danse
}

\author{
Guillaume Sintès
}

1 L'exposition Chaillot, une mémoire de la danse', qui s'est tenue pendant l'été 2018 à Paris, à la Bibliothèque nationale de France, fut l'occasion de documenter l'histoire de la présence de la danse depuis la fin du $\mathrm{XIX}^{\mathrm{e}}$ siècle et tout au long $\mathrm{du} \mathrm{Xx}^{\mathrm{e}}$ siècle, dans un théâtre qui ne lui était pas initialement dédié, jusqu'à ce que Chaillot devienne, en 2016, Théâtre national de la danse. Dans ce panorama couvrant presque 130 ans d'activités artistiques, un chorégraphe occupe une place importante: Maurice Béjart. Il serait possible, en s'appuyant sur les archives, de retracer les principales dates de la venue du chorégraphe à Chaillot ou de rendre compte de la programmation de telle ou telle œuvre au cours des dernières décennies. Mais plutôt que de proposer une histoire de la présence de Béjart à Chaillot, nous prenons le contre-pied d'élaborer celle de son absence : une anti-histoire, en quelque sorte. Toutefois, notre anti-histoire ne vient pas contredire ou s'opposer à l'histoire de la danse à Chaillot telle que construite par cette exposition. Au contraire, elle la complète comme le ferait, pour reprendre la célèbre formule de Claude Lévi-Strauss ${ }^{2}$, " une histoire faible » : c'est-à-dire une microhistoire qui, relevant du local, du biographique, voire de l'anecdotique, permettrait de révéler « les individus dans leurs particularités ». Aussi, nous appliquerons-nous à rappeler les enjeux d'un projet qui n'a jamais abouti - celui de l'installation d'une école de danse moderne qui aurait dû être confiée à Maurice Béjart, mais qui ne le fut pas parce qu'elle ne vit simplement pas le jour - et, ce faisant, à construire le récit de l'histoire qui n'eut pas lieu de Béjart à Chaillot.

2 Maurice Béjart ${ }^{3}$ commence la danse, adolescent, à l'Opéra de Marseille, puis se forme à l'Opéra de Paris au sortir de la Seconde Guerre mondiale, auprès de Janine Charrat, Yvette Chauviré et Roland Petit. Il fonde sa première compagnie, les Ballets de l'Étoile, en 1954. À cette époque, Béjart s'inscrit dans un paysage chorégraphique qui a été sculpté pendant près de trois décennies par Serge Lifar - maitre de ballet de l'Opéra de Paris, de 1930 à 1958 -, régnant de manière omniprésente et autocratique sur la danse en France. Les Ballets de l'Étoile font alors partie d'une petite constellation de compagnies néoclassiques qui s'épanouissent tant bien que mal au cours des années 
cinquante. Il s'agit pour l'essentiel de danseurs et danseuses transfuges de l'Opéra de Paris ${ }^{4}$ qui s'essaient à la chorégraphie, s'inscrivant dans la filiation ou en dissidence de Lifar. S'agissant plus particulièrement de Maurice Béjart, le chorégraphe décide en 1959, faute de moyens financiers - à cette époque, l'État français ne soutenait (en dehors de l'opéra de Paris et de quelques ballets de maisons d'opéra en province) que très marginalement la danse -, de s'établir à Bruxelles. Cette expatriation, même nuancée par une proximité géographique évidente, suscite presque immédiatement l'émoi du nouveau ministère des Affaires culturelles, créé cette même année 1959. S'ouvre alors une longue séquence de plusieurs décennies pendant laquelle se succèdent atermoiements politiques et essais infructueux des différents ministères et secrétariats d'État à la Culture qui échouent tous à faire revenir le chorégraphe en France, tandis que sa notoriété internationale va grandissante. Nous nous concentrerons sur l'un de ces échecs: le projet de l'installation de l'école de Maurice Béjart à Chaillot qui occupa les services du ministère de la Culture (ainsi que la presse) de 1975 à 1981.

3 Après la tentative ratée de confier à Maurice Béjart la direction de la danse à l'Opéra de Paris, au sein d'un triumvirat qui aurait placé aux côtés du chorégraphe, Jean Vilar et Pierre Boulez - laquelle échoua dans les remous de Mai $68^{5}$-, un projet d'envergure pour encourager le retour du chorégraphe est porté fin 1975 par Michel Guy ${ }^{6}$, secrétaire d'État à la Culture alors en poste. Amateur d'art contemporain et balletomane averti, Michel Guy a été conseiller artistique au Festival international de danse de Paris de 1964 à 1971, puis a assuré la direction du Festival d'Automne que Georges Pompidou lui a confié en $1972^{7}$, avant d'arriver rue de Valois en juin 1974.

4 Le mandat de Michel Guy est marqué par son américanophilie : ses préférences en matière de danse vont à Paul Taylor, Merce Cunningham, Alwin Nikolais. Ce goût personnel est manifeste dans la politique culturelle qu'il met en place en faveur du développement de la danse et se caractérise notamment dans l'édification d'un système économique de tournées des compagnies de danse, lequel bénéficiera prioritairement, dans un premier temps du moins - via le budget mis à disposition par l'office national de la diffusion artistique (ONDA) que Michel Guy vient de créer cette même année 1975 -, aux troupes américaines qui se produisent en France. Dans le domaine pédagogique, le modèle de la modern dance américaine fait également loi.

5 Pour s'en convaincre, il suffit de lire la conférence de presse que Michel Guy et Igor Eisner, inspecteur général de la danse (poste créé par le secrétaire d'État quelques mois plus tôt), tiennent en décembre 1975. Eisner y annonce deux mesures dont le développement important marquera les années qui vont suivre: la mise en place régulière de stages de danse fondés sur l'invitation de chorégraphes de renommée internationale, essentiellement, pour ne pas dire exclusivement, américains, et le projet d'un centre de formation et de recherche de danse contemporaine. À ces deux mesures, s'ajoute un autre point majeur, celui de l'installation de l'école de danse de Maurice Béjart à Paris :

«Maurice Béjart a quitté la France en 1959 pour la Belgique. Ce n'est pas loin mais il est assez désagréable qu'un Français célèbre vienne à Paris, une ou deux fois par an, en étranger. Béjart a créé, il y a trois ans à Bruxelles, une école de perfectionnement destinée à de jeunes danseurs et danseuses qui veulent se familiariser avec les autres disciplines du spectacle. C'est Mudra.

Maurice Béjart est disposé à diriger en France chaque été un stage de trois mois en appliquant les méthodes de travail qui ont fait le succès de Mudra pour une 
quarantaine de danseurs. Nous avons accepté cette proposition [...]. Le premier stage est prévu pour l'été 1977. Là aussi se pose un problème de locaux mais je pense qu'avec le concours d'une ou plusieurs collectivités, nous trouverons ce dont nous avons besoin. $»^{8}$

6 L'invitation ainsi faite à Maurice Béjart permet, d'une part, d'engager un processus dont l'objectif in fine est son installation durable en France, mais elle s'inscrit, d'autre part, dans la logique d'un plan de formation des danseurs français qui passe notamment par le biais de stages de danse. Mudra s'impose aussi, dans cette conférence de presse, comme un modèle pédagogique alternatif à celui de l'école américaine : créer "une école française moderne comme il y a une école française classique " ${ }^{9}$ et faire revenir en France Maurice Béjart, se mêlent ici dans un même but. Ces deux projets dont seul le premier se réalisera avec l'ouverture à l'automne $1978 \mathrm{du}$ Centre national de danse contemporaine (CNDC) d'Angers, confié à un chorégraphe américain, Alwin Nikolais - occupent une place singulière dans la politique pédagogique menée par l'État, dans cette seconde moitié des années soixante-dix. Il s'agit d'ériger, ou plutôt de réaffirmer, la figure du chorégraphe en pédagogue.

7 Cette politique culturelle chorégraphique s'inscrit dans un contexte particulier : celui de l'impossibilité d'appliquer une loi votée dix ans plus tôt, mais dont les décrets d'application tardent à venir ${ }^{10}$ (ils ne verront d'ailleurs jamais le jour). Cette loi, c'est celle du $1^{\text {er }}$ décembre 1965 qui visait à encadrer l'enseignement de la danse à destination des danseurs-interprètes amateurs et professionnels. En faisant appel à des chorégraphes de renommée internationale, le secrétariat d'État à la Culture tente ainsi d'institutionnaliser la relation maître-élève qui demeure un modèle dominant dans l'enseignement de la danse moderne (allemande comme américaine).

8 La récente nomination de Carolyn Carlson à l'Opéra de Paris, comme l'organisation des stages d'été à la Chartreuse de Villeneuve-lès-Avignon - qui accueille Cunningham en 1976, Carlson en 1977 et Nikolais en 1978 - relèvent d'actions qui visent autant la pédagogie que le prestige. Il en est de même pour les «Deux projets pour l'enseignement $\|^{11}$ que prépare le secrétariat d'État à la Culture depuis l'annonce faite en décembre 1975. À propos du projet d'une école béjartienne, une note interne indique en effet que :

«Maurice Béjart est disposé à diriger, sur le modèle de Mudra Bruxelles, une école pluridisciplinaire qui fonctionnerait en France pendant les trois mois de l'été avec quarante stagiaires. Deux emplacements sont envisagés : l'Abbaye des Prémontrés à Pont-à-Mousson et l'Abbaye de Fontevraud dans le Maine et Loire. D'abord prévu pour 1977 ce projet [...] doit être réalisé à la fin de 1976 ou au début de 1977. »

L'obstacle principal auquel l'administration est confrontée, pendant les sept années que durera le projet d'installation de l'école de Maurice Béjart, relève d'un aspect essentiellement pratique: l'incapacité de trouver un lieu d'accueil pour une école Mudra.

10 Mené parallèlement au projet du CNDC d'Angers ${ }^{12}$, le retour de Maurice Béjart en France est d'abord envisagé sous la forme de stages d'été, ainsi qu'en témoigne une note d'Igor Eisner intitulée "Installation en France de l'école MUDRA » datée de fin septembre 1976 :

« Pour différentes raisons - l'emploi du temps de Maurice Béjart, les contraintes financières, la nécessité de ne pas faire une concurrence flagrante à Bruxelles - il a été entendu que cette école serait ouverte pendant trois mois d'été, du $1^{\mathrm{er}}$ juillet au 30 septembre. 
Cependant, l'enseignement serait dispensé par les professeurs de MUDRA pendant leurs propres vacances et aurait le caractère pluridisciplinaire (danse, diction, chant, mime) qui en fait la célébrité de MUDRA. L'école devrait être conçue pour quarante stagiaires, filles et garçons recrutés sur audition sans critère de nationalité. Maurice Béjart a suggéré que le stage de trois mois pourrait s'ouvrir sur un spectacle donné par MUDRA Bruxelles et se terminer par un spectacle préparé par les quarante stagiaires de MUDRA France. Ce serait l'aspect public de l'opération. " $^{13}$

11 Ainsi qu'il en est question dans la conférence de presse de décembre 1975, plusieurs sites sont pressentis pour accueillir l'école estivale de Maurice Béjart: les abbayes de Saint-Maximin (dans le Var), de Fontevraud (dans le Maine-et-Loire) et des Prémontrés (en Meurthe-et-Moselle). Le premier site recueille l'opposition des élus locaux parmi lesquels le maire de Marseille et député des Bouches-du-Rhône, Gaston Defferre, qui juge que l'installation de Maurice Béjart risquerait de concurrencer le Ballet de Marseille dirigé depuis 1972 par Roland Petit. Le deuxième site nécessite des travaux importants de rénovation. De plus, la proximité géographique du Ballet-Théâtre contemporain (encore installé à Angers en 1975) est également avancée (selon le même argument que le Ballet de Marseille). Enfin, bien que proche de la Belgique, l'abbaye des Prémontrés pose un double problème: elle abrite depuis deux ans un concours chorégraphique international qui est le prolongement du concours de Bagnolet, célèbre concours de danse qui a permis l'émergence et la mise en lumière des chorégraphes de la Nouvelle Danse française. Cette manifestation, bien vue par la profession, n'a cependant pas encore acquis le rayonnement international souhaité et la mettre en concurrence avec Maurice Béjart pourrait lui être préjudiciable. D'autre part, les Ballets de Nancy, dirigés par Gigi Caciuleanu ${ }^{14}$, ont eux aussi le projet de s'installer à l'abbaye des Prémontrés pour des sessions de travail et de représentations chorégraphiques publiques. La note d'Igor Eisner se conclut sur le constat suivant:

«On peut penser, d'ores et déjà, que M. Béjart, s’il vient aux Prémontrés, voudra avoir un œil sur toutes les autres activités chorégraphiques réparties dans l'année et même s'il ne fait pas connaître franchement son opinion, il risque de bouder les Prémontrés après avoir donné une acceptation de principe. $»^{15}$

12 L'installation de Maurice Béjart semble se heurter aux premiers effets de la décentralisation chorégraphique : où qu'on lui propose d'aller, une compagnie de danse (bien souvent néoclassique) y est déjà installée. Cette situation étrange oblige à s'interroger: le nom du chorégraphe fonctionne-t-il comme un repoussoir? Ou le ministère de la Culture fait-il preuve de malchance avec ces choix peu judicieux? Pourtant, le retour de Maurice Béjart est une priorité ministérielle.

Les archives du ministère de la Culture, conservées aux Archives nationales, font état de nombreuses notes internes, comme celles précédemment citées, concernant tout ou partie du projet d'installation du chorégraphe en France tel qu'envisagé par Michel Guy, puis poursuivi par ses successeurs ${ }^{16}$ (notamment par Jean-Philippe Lecat), jusqu'à qu'il soit définitivement abandonné par Jack Lang en 1981. Ces documents rendent compte le plus souvent des entrevues que les agents et responsables du ministère de la Culture ont eues avec Maurice Béjart, soit à Bruxelles, soit lors de la venue du chorégraphe à Paris pour telle ou telle représentation.

Il en ressort que de nombreux efforts ont été régulièrement consentis par les services administratifs pour faciliter l'activité professionnelle de Béjart en France, à titre d'exemples : l'accueil du Ballet du $\mathrm{xx}^{\mathrm{e}}$ siècle dans la Cour carrée du Louvre, la captation 
de spectacles par la télévision publique, le financement de divers projets chorégraphiques, l'attribution de bourses en faveur de danseurs de Mudra Bruxelles... Certaines notes font le point sur les divers projets de Maurice Béjart tant en France qu'en Belgique ou à l'étranger. Et l'on peut ainsi suivre le parcours du chorégraphe sur l'ensemble de cette période, jusqu'à (presque) en recomposer l'agenda. Cette démarche témoigne, s'il en est, de l'intérêt très fortement marqué du ministère de la Culture pour le chorégraphe : «il est nécessaire de ramener Béjart en France », peut-on lire sur une note manuscrite de 1977. L'enjeu est tel que d'un projet de politique culturelle, le retour de Maurice Béjart va devenir une affaire politique.

Le projet de stages Mudra l'été devient assez rapidement celui d'un centre chorégraphique permanent, dirigé par Maurice Béjart. Une note ${ }^{17}$ de Claude Gallant, qui est alors responsable du bureau de l'Art lyrique et de la Danse, datée de 1979, en précise les modalités.

16 Le projet est celui de réunir l'école et la compagnie de Maurice Béjart en un lieu unique à Paris dès 1981. Évalué à 5,7 millions de francs ${ }^{18}$ par l'administration, sur la base d'un budget prévisionnel établi par le chorégraphe lui-même, le coût de l'opération semble démesuré au regard du reste du budget « enseignement » du ministère de la Culture ${ }^{19}$. De plus, bien que dans son projet de centre chorégraphique Béjart ne «mentionne aucun des renseignements indispensables permettant de caractériser son mode de fonctionnement (nombre d'élèves, conditions de travail des professeurs, des danseurs et des élèves, maintien éventuel d'avantages acquis au profit d'anciens salariés de Mudra, etc.) », le projet est néanmoins accepté en l'état par le ministère de la Culture.

17 L'obstacle à la réalisation de ce projet n'est donc pas financier, mais réside, une nouvelle fois, dans le lieu choisi pour accueillir le centre chorégraphique de Béjart à Paris : Chaillot.

Au printemps 1979 débute une polémique concernant l'installation de l'école du chorégraphe au Palais de Chaillot. Le 11 avril 1979, le journaliste Bernard Lartigue publie, face à face, deux articles dans L'Humanité. Le premier est titré «Misère de la danse » et le second se présente sous la forme interrogative : «Une école de danse au musée des monuments français? ». Dans le premier article, le journaliste déplore le peu d'aide du gouvernement en place pour les compagnies de danse françaises qu'il juge "en situation d'infériorité par rapport aux troupes étrangères ", il regrette aussi l'anarchie de l'enseignement de la danse, l'absence de statut pour les danseurs, le "manque de crédits attribués aux compagnies [duquel] découlent les salaires les plus bas de tous les artistes interprètes et un fort chômage ( $80 \%)$; souvent même les danseurs ne sont pas déclarés et ne peuvent toucher l'allocation. Les retraites sont ridicules (400 frs par mois) quand la carrière se déroule de vingt à quarante-cinq ans environ ». Le budget prévisionnel de l'école de Maurice Béjart ( 6 millions de francs, en légère augmentation, donc) dévoilé dans le second article ne provoque que l'accueil bienveillant du journaliste, sans même être mis en perspective de la situation «miséreuse » générale de la danse en France qu'il a analysé précédemment. Le seul problème observé par Bernard Lartigue est celui du choix du lieu d'implantation de l'école béjartienne.

19 Le palais de Chaillot construit pour l'exposition universelle de 1937, sur l'ancien palais du Trocadéro, abrite en 1979 le musée des Monuments français ${ }^{20}$, le Théâtre national de Chaillot (labélisé comme tel en 1975) et les collections de la Cinémathèque française ${ }^{21}$. Jean-Philippe Lecat, alors ministre de la Culture (mandat qu'il occupe d'avril 1978 à 
mars 1981), souhaite redynamiser le site et en faire un centre d'art contemporain destiné aux arts vivants. Devant l'échec de l'installation de Maurice Béjart en province, il fait visiter le musée tombé en désuétude au chorégraphe. Mudra occuperait ainsi l'espace du musée et bénéficierait des nouveaux aménagements du théâtre pour y produire ses ballets. L'idée enchante le chorégraphe, mais beaucoup moins la presse.

Dans un article ${ }^{22}$ paru dans le journal L'Aurore, Irina de Chikoff résume ainsi la situation:

«Béjart. Chaillot. Lecat. Un chorégraphe. Un palais. Un ministre. L'équation est anodine, mais le résultat détonnant; car il suppose l'amputation, le démantèlement, voire la suppression, du musée des Monuments français, sis dans l'aile gauche du palais de Chaillot. Ce qu'on peut d'ores et déjà appeler l'affaire du musée fait aujourd'hui des remous, mais elle date presque d'un an.

C'est, en effet, en automne 1978 que Jean-Philippe Lecat, ministre de la Culture et des Communications, rencontre pour la première fois Maurice Béjart [...]. Le ministre, tout comme ses prédécesseurs depuis Malraux, voudrait bien faire revenir à Paris le Maître, à la fois contesté et incontestable, des ballets modernes. [...]

Quelques mois plus tard, Béjart vient visiter Chaillot en tant que conseiller d'on ne sait trop quoi. [...] Il a le coup de foudre. [...] Le 3 avril dernier, toujours en catimini, une nouvelle entrevue réunit le ministre et le danseur. Rien ne devait filtrer. Rien ne devait se savoir. Mais les bruits vont vite et la menace qui pèse sur le musée conçu par Viollet-le-Duc semble se préciser. [...] Chacun connaît le peu de goût de Béjart pour les musées où il ne voit que poussière et qu'il aime comparer à des tombeaux. [...] Pas plus que vous, sans doute, je ne connaissais ce musée. J'y suis allée et j'ai été aussitôt séduite. [...]

Nul ne songe à contester le talent de Béjart. Nul ne songe à contester que son retour à Paris serait une opération de prestige rentable. Ces arguments sont-ils suffisants pour justifier la disparition, ou au "moins pire", la mutilation du musée imaginaire ? [...]

Outre les millions nécessaires à l'éventuelle installation de l'école de danse de Béjart et aux trois millions nécessaires au fonctionnement annuel de cette école, il faudrait donc ajouter les millions, nécessaires eux aussi, au déménagement des moulages du musée des Monuments. [...] D'un côté, nous avons un musée unique au monde dont le prestige pourrait être exploité et ne l'est pas. Un musée imaginaire méconnu et par conséquent peu rentable. De l'autre côté, nous avons Béjart dont le nom à lui seul attire les foules. La facilité consiste évidemment à remplacer le non rentable par le brillant. Le sérieux par le clinquant.»

Plusieurs arguments (de la protection du patrimoine à la démesure financière du projet) se mêlent dans cette démonstration à charge, mais tous concourent à s'opposer au projet d'implantation de l'école de Béjart. Une pétition est lancée par le prix Nobel de médecine André Lwoff, qui recueille plus de dix mille signatures ${ }^{23}$. L'Académie des beaux-arts s'associe à l'Union nationale de la sculpture française pour désigner un comité de sauvegarde d'un musée qui, de l'aveu de tous, n'intéressait personne jusqu'à ce qu'on décide de le déménager.

Il conviendrait sans doute d'analyser davantage la réception en France des œuvres et de la personnalité de Maurice Béjart, sans doute aussi la situation générale de la politique culturelle menée par l'État dans les autres domaines (l'architecture et le patrimoine en particulier), mais d'ores et déjà nous pouvons constater l'opposition farouche que suscite celui qui fait déplacer les foules à chacune de ses visites en France. Devant le tollé général et pour mettre un terme à la polémique, Jean-Philippe Lecat, sans totalement faire machine arrière, réplique que l'implantation de l'école de Maurice Béjart aura bien lieu à Chaillot, mais que celle-ci sera logée dans les combles du 
bâtiment qui seront, pour l'occasion, surélevés selon les plans originaux du palais ${ }^{24}$. Très vite, le ministère de la Culture doit faire face à une nouvelle opposition : celle de la Commission des Monuments historiques qui a classé fin 1980, en toute hâte, le bâtiment et rejette le projet architectural, bien que conforme, au nom d'une «question de principe $»^{25}$.

23 Ni le changement de présidence, ni la nomination de Jack Lang au ministère de la Culture ne permettront de faire aboutir le projet. Béjart renonce au projet d'un Mudra à Chaillot. Là encore, l'annulation crée une polémique dont s'empare la presse à l'automne 1981. Le ministère de la Culture et le chorégraphe se rejetant chacun la faute de cet échec ${ }^{26}$. Il est d'ailleurs assez ironique que l'abandon de l'école soit l'objet de regrets des mêmes journalistes qui s'étaient initialement et vigoureusement opposés au projet $^{27}$.

L'échec d'un Mudra à Paris bénéficiera aux autres actions de soutien en faveur de l'enseignement (dont l'essor de celui de la danse moderne) et obligera le ministère de la Culture à réactiver la refonte de la loi du $1^{\text {er }}$ décembre 1965. Plusieurs projets législatifs seront ainsi envisagés (en 1981 et $1982^{28}$ ) jusqu'au vote de la loi de 1989 (à partir d'un projet amorcé en $1987^{29}$ ). Dans un autre ordre d'idée, l'abandon de Maurice Béjart a semblé profiter à Roland Petit, dans la concurrence symbolique que se livraient les deux chorégraphes à la renommée internationale. Elle aura ainsi permis au Ballet de Marseille de voir sa dotation annuelle quadrupler entre 1972 et 1979 (passant de 300000 frs à $1242000 \mathrm{frs}^{30}$ ). Mais, cet effort d'une politique pédagogique du ministère de la Culture reste encore très largement anecdotique au regard du budget global du ministère : l'ensemble du budget, en 1978, consacré à la danse représente tout au plus $10 \%$ (y compris le Ballet de l'Opéra de Paris) ${ }^{31}$.

Sans doute parce qu'elle n'eut pas lieu, cette anti-histoire de Béjart à Chaillot permet de mieux comprendre certains des enjeux d'une politique culturelle en faveur de la danse et la réalité économique d'un domaine artistique qui, jusqu'aux premières années du ministère Lang, peine à se hisser au niveau dont bénéficient, dans les arts du spectacle, le théâtre et la musique.

\section{NOTES}

1. Du 3 mai au 26 août 2018, BnF-site François-Mitterrand, allée Julien Cain. Commissariat d'exposition : Joël Huthwohl et Valérie Nonnenmacher.

2. «L'histoire biographique et anecdotique [...] est une histoire faible [...]; mais elle est [...] riche du point de vue de l'information, puisqu'elle considère les individus dans leur particularité, et qu'elle détaille, pour chacun d'eux, les nuances de caractère, les détours de leurs motifs, les phases de leurs délibérations. Cette information se schématise, puis s'efface, puis s'abolit, quand on passe à des histoires de plus en plus 'fortes'. [...] On vérifie alors que chaque histoire faible d'un domaine inscrit est complémentaire de l'histoire forte du domaine circonscrit [...]. Chaque histoire s'accompagne donc d'un nombre indéterminé d'anti-histoires, dont chacune est complémentaire des autres : à une histoire de rang I correspond une anti-histoire de rang II, 
etc. » LÉVI-STRAuss (Claude), La Pensée sauvage, Paris, Plon, 1962, p. 346. Idem pour les citations suivantes.

3. Maurice Béjart (1927-2007), de son vrai nom Maurice-Jean Berger, français naturalisé suisse, fils du philosophe Gaston Berger, fut danseur, chorégraphe, directeur de compagnie et pédagogue.

4. C'est notamment le cas dès 1945 des Ballets des Champs-Élysées de Roland Petit - compagnie rebaptisée, trois ans plus tard, Ballets de Paris -, des Ballets Janine Charrat créés en 1951 ou encore des Ballets de la Tour Eiffel fondés par Pierre Lacotte en 1955.

5. Voir à ce sujet RAISON (Francis), «Un directeur dans la tourmente », dans ABIRACHED (Robert) [dir.], La Décentralisation théâtrale. 1968, le tournant, t. III, Arles, Actes Sud, coll. Actes Sud/Papiers, 2005 [1994], p. 61 ; SINTÈs (Guillaume), « Michel Descombey à l'Opéra de Paris : un rendez-vous manqué ? ", dans LAUNAY (Isabelle), PAGÈs (Sylviane), PAPIN (Mélanie) et SINTÈs (Guillaume) [dir.], Danser en 68. Perspectives internationales, Montpellier, Deuxième Époque, 2018, p. 230-244.

6. Michel Guy (1927-1990) fut secrétaire d'État à la Culture de juin 1974 à août 1976.

7. Voir à ce sujet DARDY-CRETIN (Michèle), Michel Guy, secrétaire d'État à la Culture (1974-1976). Un innovateur méconnu, Paris, Comité d'histoire du ministère de la Culture, 2007; ainsi que les travaux de Patrick Germain-Thomas, notamment : «La politique de la danse contemporaine en France: une construction conjointe des pouvoirs publics et des lieux de programmation", Quaderni [en ligne], $\mathrm{n}^{\circ}$ 83, hiver 2013-2014. <http://journals.openedition.org/quaderni/761>. Consulté le 6 novembre 2018.

8. EISNER (Igor), «Conférence de presse sur la Danse du Secrétariat d'État à la Culture » du 9 décembre 1975.

9. Ibidem.

10. Il serait trop long d'exposer dans ces lignes les nombreuses causes et l'ensemble des enjeux qui se sont cristallisés autour de cette loi du $1^{\text {er }}$ décembre 1965. Nous renvoyons le lecteur aux analyses de Luc ALLAIRE, «La Loi sur la danse : préhistoire », Marsyas, revue de pédagogie musicale et chorégraphique, hors-série : «1967-1997. Trente ans d'enseignement de la musique et de la danse en France ", décembre 1997 ; et Guillaume sinTès, Préfiguration, structuration et enjeux esthétiques du métier de chorégraphe (France, 1957-1984). Une histoire administrative, réglementaire et politique de la danse, thèse sous la dir. d'Isabelle Launay, Université Paris 8, 2015.

11. Ce point figure aux côtés du projet de CAP de danseur dans une note interne datée de septembre 1976 : «La Danse. Actions en cours et perspectives », document non signé.

12. Un temps, les deux projets sont même associés. Dans ses « Mesures nouvelles 77 », Igor Eisner écrit à l'article $\mathrm{n}^{\circ} 145$ : «Maurice Béjart souhaite retrouver une activité permanente en France. Le Secrétariat d'État lui a annoncé le report de l'implantation d'un Mudra d'été en 1978. L'abbaye des Prémontrés (Pont-à-Mousson) semblait le meilleur choix possible. Cependant si le Secrétariat d'État à la Culture fait l'acquisition de l'immeuble de la rue de Trévise pour le Centre Chorégraphique National, M. Béjart acceptera peut-être que le stage ait lieu à Paris. »

13. "Installation en France de l'école MUDRA », note d'Igor Eisner à l'attention de Monsieur le Directeur (sans autre précision, sans doute s'agit-il du directeur du cabinet du ministre), datée du 29 septembre 1976.

14. Gigi-Gheorghe Caciuleanu (1947-), chorégraphe d'origine roumaine arrivé en France en 1973, fonde en 1974 le Studio de danse contemporaine à Nancy. Il fut par la suite directeur du Centre chorégraphique national de Rennes de 1978 à 1993.

15. « Installation en France de l'école MUDRA », note d'Igor Eisner, 29 septembre 1976.

16. À savoir : Françoise Giroud (1916-2003) qui fut secrétaire d'État à la Culture d'août 1976 à mars 1977, Michel d'Ornano (1924-1991) qui occupa les fonctions de ministre de la Culture et de l'Environnement de mars 1977 à mars 1978, puis de nouveau par intérim de mars 1981 à mai 1981, 
ainsi que Jean-Philippe Lecat (1935-2011) qui fut ministre de la Culture et de la Communication entre les deux mandats de Michel d'Ornano, d'avril 1978 à mars 1981.

17. "Création d'un Centre chorégraphique dirigé par M. Béjart ", note de Claude Gallant à l'attention de Monsieur le Directeur du Cabinet (bien que non datée, les éléments présents dans la note nous permettent de la situer en 1979).

18. Équivalant à près de $2730000 €$ (année de conversion : 2017), selon le convertisseur franceuro disponible sur le site de l'INSEE <http://www.insee.fr/fr/themes/calcul-pouvoir-achat.asp>. Consulté le 6 novembre 2018.

19. Claude Gallant écrit dans sa note: «Par rapport à l'École de danse de l'Opéra, le centre chorégraphique de M. Béjart présente un budget presque deux fois plus élevé. Or l'effectif de l'École de Danse dépasse les 100 élèves. En revanche, elle ne dispose pas de troupe permanente et donne, en conséquence, un seul spectacle de fin d'année. La comparaison avec le centre chorégraphique d'Angers, dirigé par A. Nikolais, est plus significative puisque ce dernier dispose d'une compagnie de 10 danseurs et d'une école de 23 stagiaires. Son coût sera de 2 MF en 1979 pour les collectivités publiques qui le subventionnent. Soit une proportion de 1 à 3 au détriment $\mathrm{du}$ Centre parisien qui, il est vrai, dispense un enseignement pluridisciplinaire et donc plus coûteux. [...] Ce budget paraît à la fois incomplet et surévalué. [...] En effet, les rémunérations du directeur adjoint et du directeur technique se situent aux niveaux maximum généralement admis. Celle du régisseur et des professeurs de danse dépasse ces niveaux qui sont de l'ordre de 8000 frs mensuels pour le premier et de 5000 à 6000 frs pour les seconds (référence Opéra de Paris). Les rémunérations des danseurs sont également avantageuses. Une réduction moyenne de 1000 frs par mois peut parfaitement être pratiquée ».

20. Créé en 1879, sous le nom de musée des Sculptures, par l'architecte et défenseur du patrimoine historique français Eugène Viollet-le-Duc (1814-1879), ses collections sont réunies aujourd'hui au sein de la Cité de l'architecture et du patrimoine, toujours sise au Palais de Chaillot.

21. Créée en 1936 par Henri Langlois (1914-1977), la Cinémathèque française s'installe au Palais de Chaillot en juin 1963 grâce au soutien d'André Malraux. Elle y restera jusqu'en juillet 1997, date à laquelle elle est transférée provisoirement, à la suite d'un incendie de la toiture du Palais de Chaillot, au Palais de Tokyo puis dans une salle sur les Grands Boulevards, avant d'être installée sur l'ancien Centre culturel américain rue de Bercy dans le XII ${ }^{\mathrm{e}}$ arrondissement de Paris. 22. DE CHIKOFF (Irina), «Cette affaire du musée des Monuments français qui agite le monde des arts. Non, M. Jean-Philippe Lecat, vous n'avez pas le droit de sacrifier un musée unique au monde pour récupérer Maurice Béjart ", L’Aurore, 19 avril 1979.

23. MAZARS (Pierre), « Béjart à Chaillot : une solution en vue », Le Figaro, juin 1979.

24. Voir à ce sujet les notes internes de Jérôme Clément à Jean-Philippe Lecat datées des 14 et 20 mai 1980, dans lesquelles est décrit l'ensemble des travaux.

25. Ainsi que le rapporte Le Monde dans un article non signé du 26 juillet 1981, titré « Le projet d'école de danse à Chaillot remis en cause par la Commission des Monuments historiques » : «La Commission supérieure des monuments historiques n'a pas approuvé, lors de sa réunion du 20 juillet, le projet de transformation du palais de Chaillot destiné à accueillir l'école de danse de Maurice Béjart. [...] Aujourd'hui, la commission, tout en rendant hommage à la qualité du travail de l'architecte, ne souhaite pas que le palais de Chaillot, classé monument historique à la fin de l'année 1980, soit modifié. La surélévation, qui serait masquée en partie par des arbres, ne serait réellement visible que de l'avenue d'Eylau. Mais, pour la commission, il s'agit d'une question de principe. »

26. Voir à ce sujet : les déclarations des deux parties à l'Agence France-Presse le 6 octobre 1981, le communiqué de presse du ministère à cette même date, ainsi que l'entretien que Maurice Béjart accorde à l'écrivain François Weyergans dans Le Monde du 7 octobre 1981 (" Un entretien avec Maurice Béjart. Le saltimbanque et les ministres»), ainsi que le droit de réponse de Jack 
Lang dans ce même journal, le 8 octobre 1981 ("Après la démission de Maurice Béjart, "Le gouvernement n'a pas deux paroles" déclare M. Jack Lang »).

27. À titre d'exemple, Le Figaro publie le 6 octobre un article signé C.B., sous le titre «Polémique. Mudra à Chaillot, Béjart renonce » : « Et voilà. Maurice Béjart a envoyé hier à Jack Lang une lettre lui indiquant qu'il renonçait à créer l'école Mudra qui aurait dû s'installer sous sa direction au Palais de Chaillot l'automne prochain. [...] Il y a quelque chose de déshonorant dans cette incapacité où s'est trouvé le ministère de la Culture de rapatrier la gloire la plus évidente de l'art chorégraphique contemporain. Et cette impuissance ne date pas d'aujourd'hui. Quand voici trois ans déjà, Jean-Philippe Lecat, alors régnant rue de Valois, décida de fonder à Chaillot l'École Mudra dans des locaux aménagés au-dessus du Musée des monuments français, il se heurta à l'opposition des commissions d'architecture qui stigmatisaient toute modification aux structures, même internes, d'un Palais qui rappelons-le était inachevé en 1936. L'affaire, sans être abandonnée, fut dès lors considérablement ralentie. Le "changement" de mai dernier n'entama en rien l'inertie calculée des bureaux responsables. »

28. Il s'agit respectivement des projets de loi d'Ornano et de Lang. Voir à ce sujet ALLAIRE (Luc), "La loi sur la danse: préhistoire", art. cit., p. 275-276. Concernant plus particulièrement le projet de loi Lang de 1983, se référer au « Projet de loi sur l'enseignement de la danse présenté au nom de M. Pierre Mauroy, Premier ministre, par M. Jack Lang, Ministre de la Culture ", rapport $\mathrm{n}^{\circ}$ 1376, Journal officiel, 20 janvier 1983.

29. ALLAIRE (Luc), « La loi sur la danse : préhistoire », art. cit., p. 276-279.

30. Équivalant respectivement à près de $285000 €$ et $587000 €$. Les comptes de résultat des bilans financiers du Ballet de Marseille pour les années 1972 à 1979 sont conservés aux Archives nationales.

31. Note interne de la direction de la Musique, de l'Art lyrique et de la Danse (non signée) à l'attention de M. le Ministre, intitulée « Une politique pour la danse », datée du 17 avril 1978.

\section{RÉSUMÉS}

Faire revenir en France Maurice Béjart, tel est le credo des secrétariats d'État et ministères de la Culture depuis que le chorégraphe s'est installé en Belgique. À partir de 1975 et pendant presque 7 ans, le projet de l'installation d'une antenne de MUDRA, l'école bruxelloise de Béjart, dans une aile du Palais de Chaillot, va occuper les services du ministère de la Culture et passionner tout autant la presse et le public.

INDEX

Mots-clés : Théâtre national de Chaillot, Palais de Chaillot, Béjart (Maurice), MUDRA, danse

\section{AUTEUR}

\section{GUILLAUME SINTÈS}

Maître de conférences en danse, université de Strasbourg 


\title{
Débriefer l'assemblée théâtrale
}

\author{
Daniel Deshays
}

1 À l'instar de l'armée qui débriefe ses cadres à l'occasion de leur départ à la retraite, le théâtre, au-delà même de penser à nourrir ses archives, aurait dû écouter et sauvegarder régulièrement la parole de ceux qui, des années durant, ont conçu et assuré la qualité de chacune de ses entreprises sans en laisser de trace autres que dans leur mémoire, et ce dans toutes les disciplines qui le composent. Les entretiens que nous avons pu effectuer ont produit un matériau nouveau, il révèle la part, toujours obscure et pourtant si spécifique, de l'expérience de la construction des spectacles.

D'une manière générale, la recherche établit des relations entre présent et passé, elle considère l'archive à partir du présent des pratiques, même si les pratiques actuelles du son et la nouveauté technologique lui restent largement inconnues; la vétusté des supports d'enregistrement suffit à énoncer clairement les différences. Ce présent du théâtre en tant qu'espace de recherche a toujours été un lieu d'incertitude de formes qui s'esquissent encore et que l'archivage va, malgré lui, bientôt fixer comme écriture d'une époque. Mais les documents archivés sont fragilisés par des vides dans la connaissance de leur propre réalisation, qui serait pourtant si utile de combler pour parvenir à une meilleure lecture et écoute. Il est encore possible de mieux les documenter en collectant auprès des différents régisseurs (régie générale, régies son et lumière) les données complémentaires pour agrandir la réserve de matériaux disponibles aux futurs chercheurs.

3 L'« entretien " constitue l'un des moyens qui permet d'inscrire plus précisément une histoire de ces pratiques, à la lueur de la mémoire de ceux qui y agissent peut-être encore. Les informations recueillies permettent de mieux comprendre également les archives récoltées par le passé. L'incomplétude, les trous entre les données persisteront néanmoins, le sonore lui-même se tenant dans cette paradoxale évidence, celle d'être un objet qui, même durant son écoute, demeure toujours absent et conséquemment, le souvenir déjà incertain d'un événement qui vient juste de se produire.

4 L'archive offre à celui ou celle qui l'aborde moins de données que d'interrogations. Ce « reste », qui constitue l'archive ne contient hélas que les résiduelles figées de ce qui a existé et que l'on relie entre elles de manière incertaine. Cette incertitude a toute 
raison d'exister tant ce qui est devenu l'archive a été lui aussi tenu de son vivant dans le flux quotidien des ajouts ou des repentirs du travail théâtral.

5 Programmes, brochures, dessins, photographies, maquettes, partitions, conduites de régie ou bandes-son : les objets déposés disent tant de choses, ils disent tout et pourtant si peu de ce que l'on y recherche : le vivant du théâtre. Car le théatre est un corps amibien dont la construction avance de manières parallèles et parfois opposées, entre son, scénographie, lumière et jeu d'acteur. Il se constitue devant nous mais travaille encore derrière sans que rien de ce tangible ne représente une certitude d'existence finale. Il faudrait considérer ces « restes » que sont les archives comme autant de traces métaphoriques de cet imaginaire collectif bâti devant nos yeux et nos oreilles. Tout dans l'archive est métaphore, non seulement parce qu'elle se tient hors du vivant, mais parce que le théâtre est mémoire interprétative du monde.

6 L'objet archivé parle de lui-même bien sûr, mais s'il parle, c'est de tout autre chose que de ce qu'il apportait dans la représentation. Pour simple exemple : la bande son d'un spectacle, relue comme archive, dit tout autre chose que ce que le spectacle lui permettait de dire. Ce que ses sons évoquent n'ont valu, et ce strictement, que dans l'expression variable et fragile d'une diffusion théâtrale, dans une localisation scénique précise, confronté aux corps et surtout à ces «voix étalon» de l'échelle sonore humaine, en regard desquelles ces effets prennent sens dans des infinies nuances (de niveau, de localisation, etc.).

7 Ce que l'archive permet de consulter, c'est un matériau que nous reconstruisons, chacun à l'aide de sa propre mémoire, quelle qu'elle soit : écouter c'est se souvenir de ce dont on ne se souvenait plus. Consulter l'archive, aussi vivante soit-elle, c'est se souvenir de ce que l'on n'a pas connu ou déjà quasi oublié et reconstruit, au point que ce qui apparait ne semble plus en relation avec ce reste de sensations qui demeure pourtant profondément ancré en nous. La réécoute de mes propres archives m'en dit plus sur leur origine sonore - l'instant de leur prise de son - que sur leur emploi théâtral. C'est un autre objet, que l'on maintient en soi, mais qui porte encore pourtant toute la chaleur ressentie de son vivant. L'archive est donc un nouvel objet, celui qui, comme tout objet regardé par un historien, permet de construire une «vision » autant qu'une "écoute ", nouvelle, renouvelée d'ailleurs à chaque génération, une fois oubliés les codes de vie d'une époque qui furent pourtant alors si puissants et communs à tous, et qui furent surtout l'essence de leur raison d'être.

8 La recherche offre le sentiment de confrontation à un présent foisonnant, dans le vertige de tant d'époques à revivre. Là se trouble la lucidité du manque qui se constitue à ses côtés. C'est un manque de présent, un présent qui toujours fait défaut, car augmenté du fait de la nature même du sonore qui toujours apparait en tant qu'absence.

9 Les travaux de ceux qui, chaque soir ont régi le son, la lumière, les costumes ou le plateau des théâtres, nous demeurent parfaitement inconnus. Non seulement ils sont toujours inconnus des spectateurs, mais ils le sont tout autant de la majeure partie de ceux qui collaborent à un même spectacle. Les relations que chacun entretient avec ses propres outils, comme avec les procédures que ces outils lui imposent, représentent pourtant une part essentielle des données qui, de manière souterraine, organisent l'évolution des conditions d'existence du théâtre. Il est de ce fait facile de comprendre combien il importe que la parole des praticiens advienne et qu'elle soit entendue et 
recueillie. Chacun, tentant de dire ce qu'il ou ce qu'elle a produit dans son propre travail, révèle à soi-même ce qu'il n'a jamais eu le temps de conceptualiser.

Débriefer le collectif théâtral depuis le porteur de projet jusqu'au dernier spectateur n'est pas chose facile.

11 Recueillir cette parole nécessite une écoute particulière, une écoute complice, qui alimente la parole de l'autre, devance et débusque les oublis. C'est une écoute déjà savante, riche de la connaissance des pratiques qu'il est nécessaire de tenir pour parvenir à pénétrer ce qui, dans le flou des souvenirs, tente de s'énoncer.

12 Le matériau obtenu a besoin d'être rapporté à l'histoire des pratiques. Aussi étanches soient-elles entre elles, celles-ci font toujours appel, malgré tout, à une transversalité, car le théâtre ne se construit que dans son interdépendance, sous des synchronismes flottants reliant les organes de son corps en jeu.

13 Collecter les mémoires des expériences du théâtre est ce qui manque. Cet objet si absent des archives est ce que nous livrent les entretiens réalisés avec ceux qui, des années durant, ont créé, dans ce collectif de mémoires que représente l'assemblée théâtrale.

14 Je veux vous présenter deux extraits des matériaux recueillis. Ils n'apparaissent pas comme le résultat d'une enquête, ils sont simplement riches de l'inattendu qu'une question " approximative » fait apparaître chez celui que l'on écoute. C'est finalement dans la conduite incertaine de l'entretien que le moindre objet recherché révèle sa tentaculaire dimension, qui délivre autant de pistes nouvelles dont certaines, hélas, ont été définitivement closes par la maladie de l'oubli propre à la vieillesse ou par la mort.

Le premier entretien, je l'ai effectué avec Raymond Burger, en mars 2016. Raymond Burger, qui a reçu une formation en électromécanique et électronique, a sans doute été le premier régisseur son dans l'histoire du théâtre français. Il est arrivé au CDE (Centre dramatique de l'Est) à Strasbourg en 1963 et a travaillé dans cette institution, devenue le TNS (Théâtre national de Strasbourg), toute sa carrière, jusqu'en 2003 : engagé au service Éclairage (de 1963 à 1968), responsable du service Son Cinéma (de 1968 à 1995), responsable de la formation des régisseurs à l'École du TNS (de 1995 à fin 2002) - et comédien avec les metteurs en scène Jean-Louis Martinelli (en 1995) et Stéphane Braunschweig (en 2002).

16 L'entretien porte, entre autres sujets importants (comme la formation et son activité de formateur), sur l'équipement du théâtre de Strasbourg en matériel son et la situation exceptionnelle qui est la sienne, de ce point de vue, dans les années 1970.

Il est évident qu'à partir du moment où un théâtre était équipé d'un matériel conséquent il fallait une personne sachant s'en servir. J'ai eu la chance d'être là au bon moment, le théâtre et sa technique m'intéressaient au plus haut point. Je me suis préparé à ce poste en dévorant livres et documentations sur les techniques du son, en assistant à plusieurs prises de son à Radio Alsace qui, tous les dimanches, diffusait un concert avec son orchestre philarmonique. Puis des stages en Acoustique architecturale à l'INA.

Au début du début, que ce soit pour le son ou la lumière, c'était le metteur en scène qui décidait de tout : la nature du son, le niveau, la spatialisation, si on disposait du matériel pour le faire. Très souvent, je l'ai constaté en tournée, on avait mis en place un régisseur son sans qualification spécifique, les metteurs en scène ou directeurs techniques pensant que du moment qu'on avait une chaîne audio ou une cassette chez soi on pouvait maitriser l'installation sonore... Petit à petit, il a fallu convaincre en proposant des effets ou des ambiances sonores qui pouvaient amener 
un plus à la perception de la scène. Cela a mis un peu de temps, certains metteurs en scène se sentaient démunis ou dépossédés. Mais rapidement, ils ont compris ce que la technique pouvait leur apporter. Le magnétophone portable professionnel nous a permis d'aller enregistrer des sons de la nature ou dans des endroits spécifiques. Il m'est arrivé fréquemment de passer une bonne partie de la nuit dans une forêt, ou bien au bord du Rhin, enregistrant les passages de péniches, ou bien encore dans une casse automobile, tapant à plusieurs sur une carcasse de voiture...

Souvent il fallait travailler le son avant diffusion. Le son théâtre n'a rien à voir avec le son cinéma. Pour vous en convaincre, enregistrez la bande-son d'un western par exemple ; écoutez la bande deux jours après avoir vu le film, vous ne serez pas déçu du résultat. Au cinéma, on voit la pluie, au théâtre non. Au théâtre, si vous diffusez le son de la pluie vous aurez l'impression d'entendre une friture. Au théâtre, on ne voit pas, alors il faut que l'imaginaire fasse le travail. Souvent, avec un léger ralenti, on arrive à " grossir » légèrement ou bien on rajoute un léger son d'écoulement pour de suite penser à de l'eau... C'est pareil pour tout ce qui est sons d'ambiance, il faut rajouter un petit élément qui dirige notre pensée. Le travail du son au théâtre, c'est comme de la bonne cuisine. Il y a un fond puis des ingrédients pour relever le tout. La multidiffusion permet de donner du relief au paysage sonore. Imaginez un orage violent qui commence par un vent violent qui sera diffusé partout, salle et plateau, un bruissement de feuilles dans les arbres, puis la pluie arrive - imaginons qu'elle arrive du lointain vers la salle - enfin le tonnerre sera diffusé à des endroits précis d'où la nécessité de la spatialisation [...].

17 Le second entretien, je l'ai effectué avec Didier Monfajon en décembre 2016, dans son bureau de la Comédie-Française. Il occupa durant trente années, de 1978 à 2008, les postes de régisseur de scène, régisseur général, puis directeur technique dès 1982 au Théâtre de Chaillot puis au Français. On y apprit qu'il avait suivi en 1973-1974 les cours de l'École de la rue Blanche, dans laquelle n'existait encore ni enseignement technique de la lumière, ni enseignement technique du son...

DD : Il y avait des créations de bandes-son à chaillot?

DM: Non. Avec Savary tout était en direct, c'était surtout de la sono, de l'amplification des voix des comédiens.

DD : Avec Vitez et Aperghis, il y avait de la musique de scène?

DM : Non pas tant que ça, parce que les Hugo [Hernani et Lucrèce Borgia, 1985], c'était de la création de bandes et avec Aperghis c'était de la musique enregistrée en studio. En direct, on n'a fait que le Monteverdi [Orfeo, 1982], avec un orchestre en scène à Gémier. Sur Le soulier de satin [1987] on avait un harmonium. On lui avait motorisé les pédales. Il fallait trouver la bonne vitesse pour ne pas trop gonfler le soufflet ! Il avait fait toute la tournée et on n'employait pas d'amplification pour lui. DD : Vous faisiez l'archivage de tous les spectacles?

DM : Pas systématiquement. Il y a eu l'archivage des bandes de spectacle. Mais la captation, ça n'était pas forcément suivi. J'ai commencé l'archivage vidéo en 1979 sur un spectacle de Victor Garcia avec une caméra N \& B. On a dû commencer à enregistrer les spectacles d'Antoine [Vitez] l'année après Faust, en 1982, et après, c'était selon les moyens financiers dont on disposait, mais ça se faisait en « démonté » : on amenait les caméras qu'on louait le soir et on les rendait vers cinq heures du matin. Nous n'avons pu louer un car régie vidéo qu'en 2000. Une partie des archives sonores d'Antoine est allé à la BnF dans les années 90 et pour Jérôme [Savary] on ne l'a pas fait.

DD : La prise de son d'archivage était faite comment ? Où se trouvaient les micros? DM : Ils étaient en fond de salle, à une distance infernale. Après on les a placés de chaque côté du cadre de scène sur des passerelles. Des micros canon. Pour Savary, on avait des PZM placés au sol sur des mousses pour reprendre les voix en comédie musicale. Mais pas de HF. On a eu juste des HF à main sur Ubu [1985] puis on a acheté des micros cravate et des émetteurs Sennheiser Diversity vers 87-88.

$\mathrm{DD}: \mathrm{Tu}$ as quitté Chaillot en quelle année? 
DM : en 2008, après presque trente années!

\section{BIBLIOGRAPHIE}

Pour une écriture du son, Éditions Klincksieck, Paris, 2006

Entendre le son, Éditions Klincksieck, Paris, 2010

Sous l'avidité de mon oreille, Éditions Klincksieck, Paris, 2018

\section{RÉSUMÉS}

À l'instar de l'armée qui débriefe ses cadres à l'occasion de leur départ à la retraite, le théâtre, audelà même de penser à nourrir ses archives, aurait dû écouter et sauvegarder régulièrement la parole de ceux qui, des années durant, ont conçu la qualité de chacune de ses entreprises sans en laisser de traces autres que mémorielles, et ce dans toutes les disciplines qui le composent. Les entretiens que nous avons pu effectuer au Palais de Chaillot ont produit un matériau nouveau qui révèle la part toujours obscure et pourtant si spécifique de l'expérience de la construction des spectacles.

\section{INDEX}

Mots-clés : Théâtre national de Chaillot, Palais de Chaillot, mémoire théâtrale, mémoire du spectateur, entretiens

\section{AUTEUR}

DANIEL DESHAYS

Études et pratiques du sonore, membre du projet ANR ECHO 


\title{
L'utopie d'une cité du tout-théâtre
}

\author{
Brigitte Joinnault
}

1 Dans l'histoire des rapports entre les lieux et les pratiques scéniques, le Palais de Chaillot fait en France figure de cas exemplaire. Comment dans un bâtiment aussi démesuré, à l'architecture monumentale et à l'entretien onéreux, conduire un projet théâtral d'intérêt national? Comment y vivre, comment y travailler, comment y recevoir le public? Les multiples problèmes posés par l'édifice lui-même ont provoqué l'imagination et l'inventivité de ceux qui y ont été confrontés. Les uns, convaincus que le lieu devait être transformé, ont tenté de persuader l'État de la nécessité d'entreprendre de lourds travaux visant à faire évoluer l'outil en le rendant plus adéquat au développement des projets artistiques qu'ils étaient missionnés pour mettre en œuvre. Certains y sont parvenus: Georges Wilson a obtenu l'édification d'une nouvelle salle de 531 places essentiellement dédiée à la création de textes contemporains, la salle Gémier inaugurée en 1967, Jack Lang, l'octroi de moyens financiers suffisants pour remanier radicalement la grande salle dont la jauge a été réduite à 1250 places (inauguration en 1974), et Didier Deschamps, la reconstruction complète de la salle Gémier qui compte maintenant 390 places (inauguration en septembre 2017) ainsi que le réaménagement de la salle de répétition, devenue studio Maurice Béjart, et essentiellement dévolue à l'accueil de projets de recherche et d'expérimentation. Les autres, plutôt que de chercher à obtenir des remaniements architecturaux majeurs, ont opté pour une politique du «faire avec", cherchant à concevoir et à mettre en œuvre des projets d'occupation compatibles avec les lieux qui leur étaient proposés, intégrant les contraintes d'espace comme des données avec lesquelles inventer.

2 Vitez, comme directeur, fait partie de ceux qui ont tenté de concevoir leur projet en fonction de l'outil dont ils héritaient et de se servir au mieux des espaces préexistants. En 1978, doutant des choix architecturaux entrepris à Ivry-sur-Seine pour doter la ville d'un théâtre, il repensait aux grands travaux entrepris par Jack Lang quelques années plus tôt à Chaillot: "Avec le recul du temps, je me dis que, dans la rénovation si controversée de Chaillot, le seul aspect critiquable, au fond, c'est d'avoir transformé cet édifice en abri. C'était aussi avouer qu'il n'y avait pas de metteur en scène. Du coup, on dut prévoir à l'avance le maximum de possibles dans l'occupation de l'espace ${ }^{1} . .$. » 
Quelques mois plus tard, c'est justement en metteur en scène « au sens noble du mot, qui implique la synthèse de l'artiste et du savant ${ }^{2}{ }^{2}$ qu'il projetait d'utiliser le lieu selon ce qu'il avait identifié comme les deux fonctions possibles d'un bâtiment: l'abri ou l'édifice.

3 Nous proposons ici de faire un point, inévitablement partiel et incomplet, sur la politique d'Antoine Vitez à Chaillot envisagée sous l'angle du rapport entre les lieux et les pratiques. Après un bref rappel de l'histoire de ses trois « passages » au Trocadéro, nous nous fonderons sur ses écrits pour revenir sur ce que furent les grandes orientations de son projet de direction. Puis, prenant appui sur des témoignages, nous pointerons l'importance du changement d'échelle dans le passage d'Ivry-sur-Seine à Chaillot, pour enfin, à partir d'un examen des programmes publiés dans les journaux du théâtre, proposer de considérer les sept saisons de sa direction comme une tentative de mettre en œuvre l'utopie d'une cité du tout-théâtre.

\section{Trois fois Chaillot}

Plagiant le titre Trois fois Électre, rappelons qu'Antoine Vitez, comme artiste salarié, est passé à trois reprises à Chaillot : dans les années 1950, comme acteur dans la troupe du TNP de Jean Vilar, dans les années 1970, comme codirecteur artistique aux côtés de Jack Lang, et dans les années 1980, comme directeur, nommé par le ministre Jean-Philippe Lecat sous la présidence de Valéry Giscard d'Estaing.

$5 \quad$ L'histoire commence par un malentendu. Selon les souvenirs de Roland Monod au sujet des liens d'Antoine Vitez avec le courant du théâtre populaire et de la décentralisation théâtrale ${ }^{3}$, alors que Jean Vilar, sans avoir de rôle à lui confier dans l'immédiat, engage Antoine Vitez en mars 1957 dans la troupe du TNP 4 parce qu'il le sait en difficulté, le jeune acteur, qui a alors 27 ans, s'attend à jouer aussitôt, et, déçu, quitte la troupe quatre mois plus tard ${ }^{5}$ sans avoir participé à aucun de ses spectacles.

Quinze ans plus tard, il revient pour travailler au Palais comme directeur artistique du nouveau Théâtre national de Chaillot dont Jacques Duhamel confie la direction à Jack Lang, après avoir transféré en mars 1972 le sigle du TNP au théâtre de la cité de Villeurbanne dirigé par Roger Planchon. L'aventure est interrompue en juin 1974 lorsque Michel $\mathrm{Guy}^{6}$, tout juste nommé secrétaire d'État à la Culture du premier gouvernement Jacques Chirac formé après l'élection de Valéry Giscard d'Estaing, décide de remplacer Jack Lang par André-Louis Perinetti. Pendant ce deuxième passage à Chaillot, Antoine Vitez participe à la création de l'éphémère Théâtre national des Enfants et met en scène deux spectacles à partir de textes non dramatiques. Le premier en mai 1973, cosigné avec Michel Raffaëlli, à partir des deux romans de Michel Tournier, Vendredi ou les limbes du Pacifique et Vendredi ou la vie sauvage, est créé sous un chapiteau installé devant le château de Vincennes pendant que le palais est en travaux. Le second, Les Miracles d'après L'Évangile de Jean, est présenté la saison suivante dans la salle Gémier, malgré la désapprobation du ministre Maurice Druon qui a tenté de s'opposer au projet de mettre en scène la Bible dans un établissement public.

7 Le troisième volet de l'histoire d'Antoine Vitez à Chaillot commence pendant l'été 1979, lorsque Jean-Pierre Angremy le sollicite pour prendre la direction du théâtre. Il réunit alors un ensemble de réflexions dans une note, «Positions et propositions pour le Théâtre national de Chaillot ${ }^{7}$ », qui sert de base pour une mission d'étude que lui confie Jean-Philippe Lecat, ministre de la Culture et de la Communication du troisième 
gouvernement Raymond Barre. La décision ministérielle est annoncée en octobre 1979 et le décret, qui prévoit une prise de fonction au $1^{\text {er }}$ juillet 1981, paraît le 18 mars 1981. La nomination d'Antoine Vitez à la direction du Théâtre national de Chaillot n'est donc nullement le fait de Jack Lang, ministre de la Culture du premier gouvernement Pierre Mauroy formé par François Mitterrand après son élection de mai 1981.

Les premières idées que formule Antoine Vitez quant à une éventuelle nomination à Chaillot sont de trois ordres : s'inscrire dans la tradition des directeurs artistes, à la suite de Firmin Gémier, de Jean Vilar et de Georges Wilson; penser que chaque théâtre national doit avoir une vocation bien distincte et que celle de Chaillot pourrait être précisément celle de la recherche de formes nouvelles pour un rapport nouveau avec un public nouveau, autrement dit d'être un lieu où l'on pourrait rêver de donner aux hommes des modèles; projeter de « bâtir une cité du théâtre, épousant ainsi l'utopie du bâtiment et de toute la colline » en imaginant utiliser le lieu « dans l'entièreté de son volume et de ses fonctions ${ }^{8}$.»

\section{Réflexions sur le projet de cité du théâtre}

Cette utopie, ce rêve d'une cité idéale, Vitez, dès ses premières notes de l'été 1979, l'envisage selon trois axes : l'espace, les langages et l'organisation du travail.

Sur le plan de l'espace, «tout doit être utilisé, animé, par l'esprit du théâtre : la petite salle, les foyers, les escaliers, la grande salle, l'esplanade au-dehors ou le jardin » (É5 138) écrit-il, et pour la grande salle, il imagine une utilisation en carré ou en couloir afin d'éviter le rapport frontal, qui lui semble rappeler les temps d'avant des travaux réalisés par Jack Lang et risquer d'éveiller une nostalgie des spectacles de Jean Vilar.

11 Sur le plan des langages, le projet proposé passe par une volonté de diversité, pas une diversité linéairement ordonnée dans le temps, les pratiques se succédant les unes aux autres, mais une "diversité simultanée", les pratiques cohabitant et se fécondant mutuellement : "plusieurs catégories de théâtre seront à la fois pratiquées : grand théâtre de répertoire, théâtre de recherche, théâtre pour les enfants, théâtre de marionnettes, théâtre lyrique, étude" (É5 138). Marie Étienne, dans En compagnie d'Antoine Vitez, cite des notes préparatoires datées du 15 août 1979 dans lesquelles sont inventoriées quatre-vingt-deux idées de spectacles ${ }^{9}$ parmi lesquels des tragédies, drames et comédies d'auteurs dramatiques du passé (Molière, Racine, Marivaux, Hugo, Feydeau, Courteline, Shakespeare, Goethe, Gogol, Maïakovski, Boulgakov, SouhovoKobiline), des œuvres de poètes dramatiques vivants (Césaire, Planchon, Vinaver, Pommeret, Lepoutre, Gazzah, Beckett, Kundera, Ben Jelloun, Aragon, Tsirkas, Ristos), des projets pour les marionnettes (reprendre le répertoire classique du théâtre de marionnettes, constituer un répertoire des classiques du monde - Don Juan, Hamlet, La Mort de Danton -, faire écrire des opéras contemporains), des clowneries, des satires, des films, des œuvres musicales. «Positions et propositions » définit également cinq traits caractéristiques destinés à être des constantes directrices des programmes de Chaillot: "l'idée philosophique» (selon laquelle "le théâtre est un des lieux de la philosophie»); «le portrait » (le fait de concevoir le théâtre comme « un art votif ») ; «la recherche des cultures lointaines » et l'effort de charité dans la représentation d'autrui ; « la morale du théâtre " (peu de différences de salaire et une politique de répertoire et d'alternance, autrement dit une forme de fidélité dans l'engagement des artistes), "la fonction pédagogique d'un grand théâtre » (l'idée que le théâtre rassemble et « fait école ») ${ }^{10}$. 
Sur le plan de l'organisation du travail, les réflexions d'Antoine Vitez concernent aussi bien le personnel administratif et technique, que le personnel artistique, et l'équipe des tout-proches avec qui il désire penser/réfléchir/partager les doutes et les questions sur les grandes orientations du projet. Pour le personnel administratif et technique, Pierre Vial lui conseille dès 1979 de se rapprocher de Georges Goubert, directeur administratif d'André-Louis Perinetti depuis 1976. Antoine Vitez demande à Bernard Coutant, administrateur du Théâtre des Quartiers d'Ivry (qui sera par la suite administrateur de Chaillot, Georges Goubert devenant responsable des programmes), de recueillir des informations sur les statuts, le fonctionnement, tandis que lui-même s'informe de la question du personnel, des effectifs, des rôles. Très vite, le 10 septembre 1979, il écrit à Jean-Pierre Angremy: «je ne veux pas de mise à pied, retrait d'emploi, disgrâce ; paraître un candidat sans loyauté ${ }^{11}$ ", faisant de cette exigence une condition de son projet. Pendant l'année 1980, il rencontre plusieurs membres de l'équipe technique. Dans des notes un peu plus tardives, entre la sollicitation de l'été 1979 et l'annonce de la nomination en octobre 1979 (mais peut-être existe-t-il des notes antérieures), il décrit en trois cercles la manière dont il souhaite s'entourer pour penser et mettre en œuvre son projet: un cercle exécutif constitué par une secrétaire générale, Marie Étienne, un administrateur, Bernard Coutant, un directeur technique, Gérard Poli, un cercle de ministres, « le conseil de Chaillot», avec Pierre Vial pour les relations avec la troupe, Georges Aperghis pour la musique, Yannis Kokkos pour l'image et Agnès Van Molder pour les marionnettes, puis le cercle des conseillers avec notamment Danielle Sallenave, Georges Banu et François Regnault ${ }^{12}$.

\section{L'arrivée à Chaillot, un changement d'échelle}

Le $1^{\mathrm{er}}$ janvier 1972, quelques mois après la mort de Jean Vilar (mai 1971) et avant d'être appelé à Chaillot par Jack Lang, Vitez qui a obtenu l'accord de la municipalité d'Ivrysur-Seine pour y implanter et y développer une activité théâtrale, crée le Théâtre des Quartiers d'Ivry, avec six proches collaborateurs réunis en coopérative ${ }^{13}$. Il n'y a alors aucun bâtiment dédié équipé pour le théâtre dans la ville. Quelques espaces de bureaux sont aménagés dans une maison de la rue Raspail, les ateliers de farce et de tragédie et les répétitions ont lieu dans l'ancien salon-salle à manger. Puis des préfabriqués situés dans la rue Marat hébergent l'Atelier d'Ivry et servent de local de répétition (notamment pour les 4 Molièr $^{14}$ et pour Bérénice ${ }^{15}$ ). Les spectacles, quant à eux, sont, jusqu'à l'hiver 1980, présentés dans divers lieux: salles de réunion, halls, réfectoires, préaux, gymnases, bains-douches, salles des fêtes. Plus tard une salle est louée, baptisée le Studio, au 21 rue Ledru-Rollin, elle sert notamment au festival du Printemps à Ivry créé en 1979.

14 En 1976 la municipalité d'Ivry-sur-Seine décide de construire un "vrai » théâtre de 400 places dans une grange horticole de la rue Simon-Dereure. L'inauguration a lieu en mars 1980 avec la mise en scène du Revizor. Au moment où Vitez quitte la ville, son équipe qui dispose depuis à peine plus d'un an du nouveau théâtre, n'a pas encore pris l'habitude de travailler dans un lieu unique et centré. Pour elle, l'aventure du TQI demeure celle du théâtre dans des espaces dispersés, bâtis à d'autres fins. C'est dans cet esprit qu'elle arrive en août au Palais de Chaillot pour finir les répétitions de la trilogie sur l'enfer ${ }^{16}$, entamées en juillet au Studio d'Ivry ${ }^{17}$. 
Le passage du TQI au TNC est un considérable changement d'échelle, aussi bien dans la taille de l'équipe administrative et technique, de quelques salariés seulement à Ivrysur-Seine à une centaine à Chaillot (environ pour moitié à l'administration, pour moitié à la technique), que dans le volume des espaces. Marie Étienne décrit à quel point le bâtiment paraît immense et mal entretenu. Les couloirs et les espaces de circulation sont encombrés, les câbles électriques en complet désordre ${ }^{18}$. Les quelques souvenirs que j'ai recueillis in situ auprès de compagnons d'Antoine Vitez qui ont vécu cette " arrivée à Chaillot » parlent de la mauvaise fréquentation du lieu, des vols, des chats sauvages, du bruit incessant des skateboards, et surtout des odeurs: celle de la moquette, celle de l'humidité, celle du manque d'aération, celles qui passent sous des portes du bâtiment qui servent de petits coins aux passants, celles de la cuisine qui montent des galeries souterraines ${ }^{19} \ldots$

\section{Le tout-théâtre}

Un avant-numéro de Chaillot, le journal du Théâtre national de Chaillot, paru en mai 1981, présente un préprogramme lacunaire qui met l'accent sur l'occupation des lieux et distingue, pour le hors salle, deux espaces : le Grand Foyer, associé aux marionnettes, et les lieux insolites, associés aux formes brèves et expérimentales (figure 1).

Le premier calendrier de la saison, publié le $1^{\text {er }}$ juillet 1981 dans le numéro un de Chaillot dont le rédacteur en chef est Michel Cardoze, présente également la programmation par lieu en distinguant quatre ensembles : "Grand théâtre », « Théâtre Gémier ", "Grand Foyer - Marionnettes » et "Autres lieux - Formes brèves » (figure 2). Dans la grande salle sont annoncées trois mises en scène d'Antoine Vitez présentées en alternance dans une même scénographie de Yannis Kokkos (la trilogie de l'enfer) et deux spectacles invités : Penthésilée de Kleist mis en scène par André Engel et Les Géants de la montagne de Pirandello mis en scène par Georges Lavaudant. Dans la salle Gémier sont programmées deux mises en scène d'Antoine Vitez, également présentées dans une même scénographie, signée cette fois par Claude Lemaire, Hippolyte de Joseph Garnier et L'Orfeo de Claudio Monteverdi, une création de Jérôme Deschamps, En avant !, et une mise en scène de Stuart Seide du Songe d'une nuit d'été. Les spectacles présentés hors salle sont quant à eux répartis en deux groupes. L'ensemble "Grand Foyer Marionnettes" réunit "Faust en marionnettes ", "Le Théâtre du Petit Miroir (marionnettes chinoises) », et "Nacer Khémir raconte Mille et Une Nuits " tandis que l'ensemble "Autres lieux - Formes brèves» regroupe un spectacle de Dominique Valadié et Élisabeth Catroux, Sous le lustre, une mise en scène d'Antoine Vitez présentée comme la première d'une série de portraits, Entretien avec Saïd Hammadi d'après Tahar Ben Jelloun, des concerts de luth de Hamid Meshabi, Les Petites Filles modèles d'après la Comtesse de Ségur, La Voix humaine de Jean Cocteau et Francis Poulenc mis en scène par Antoine Vitez avec Anne Béranger et Setrak, et de nouveau « Le théâtre du Petit Miroir (marionnettes chinoises) » et « Nacer Khémir raconte Mille et Une Nuits ». Le fait que les séances de conte de Nacer Khemir et de marionnettes du Théâtre du Petit Miroir, qui se donnent tantôt pour les jeunes spectateurs, tantôt pour le tout public, se retrouvent annoncés à la fois dans le grand foyer et dans les autres lieux, à la fois comme formes brèves et comme spectacles de marionnettes, fait apparaitre les difficultés engendrées par la volonté de mettre au premier plan la logique de localisation, et de systématiser l'association des pratiques et des lieux. 

traduisent assez précisément la conception du rapport au lieu théâtral exposée dans «L'abri ou l'édifice» en annonçant des localisations pour le travail en salle («Grand théâtre » et "Théâtre Gémier ») et des catégories de pratiques pour le travail hors-salle ("Formes brèves» et «Marionnettes»). À partir du numéro 4, la logique spatiale réapparaît au premier plan avec, pour le hors-salle, deux catégories, «Grand Foyer • Formes brèves » et " Grand Foyer • Théâtre pour les jeunes spectateurs », la désignation «Grand Foyer » devenant dès lors une expression unique pour nommer le hors-salle (figure 3). La distinction des pratiques évolue également et tient compte de l'âge du public avec, d'un côté, les «formes brèves » qui réunissent du théâtre-document, des marionnettes, du conte et des concerts, de l'autre, les spectacles "pour les jeunes spectateurs ». Dans l'annonce des spectacles de marionnettes de la compagnie Théâtre du Petit Miroir ce n'est plus, comme dans les journaux précédents, le nom de la compagnie suivi d'une parenthèse précisant marionnettes chinoises qui est mis en avant, mais le titre spécifique de chacun des spectacles, Le Sac du Palais du Ciel, présenté comme une forme brève jouée en chinois, et Souen Wu Kong, roi des Singes, présenté comme un spectacle pour les jeunes spectateurs, joué en français. Pour les contes de Nacer Khemir le problème des précédentes présentations a disparu et l'on comprend bien que, sous le même titre, le conteur raconte alternativement pour des publics différents. En revanche, probablement à la suite d'une erreur sur le corps du texte, une incohérence demeure sur la présentation des «Lectures de poésie » qui se retrouvent visuellement dans l'ensemble «Théâtre pour les jeunes spectateurs ${ }^{20}$. Cette incohérence disparaît dans le numéro suivant par une simple augmentation de la taille des caractères. Foyer • Formes brèves" se confirme, «Grand Foyer • Théâtre pour les jeunes spectateurs " redevient "Grand Foyer - Marionnettes » et «Lectures de poésie » apparaît comme une catégorie en soi qui n'est rattachée à aucun lieu spécifique, ce qui traduit bien le caractère nomade d'une activité errante qui s'installe en différents endroits (figure 4). Cette fois, la distinction des pratiques domine, au détriment de celle des publics. Un certain déséquilibre demeure néanmoins entre les ensembles « poésie » et "marionnettes", qui renvoient à des types de pratiques, et celui, plus flou, des « formes brèves », qui regroupe La Voix humaine et Les Petites filles modèles.

L'instabilité de la présentation se poursuit dans les journaux de la saison suivante, 1982-1983. Le fait que des spectacles de marionnettes soient programmés dans la salle Gémier et que tous les spectacles pour les jeunes spectateurs ne soient pas de marionnettes conduit à revenir sur les logiques antérieures. Le numéro 7, du mois de juin 1982, reprend la distinction " Grand Foyer • Théâtre pour les jeunes spectateurs » et "Grand Foyer - Formes brèves ", du numéro 4, tandis que le numéro 8 , du mois d'octobre, adopte la même présentation que le numéro 5. Du numéro 9 (décembre 1982) au numéro 12 (juin 1983), l'affichage de la localisation disparaît de nouveau pour le hors-salle et la présentation se fait par familles de pratiques. C'était déjà le cas dans les numéros 2 et 3 de la saison 1981-82, mais ces numéros ne distinguaient que deux ensembles, alors que ceux-ci en font apparaître beaucoup plus (" musique », "formes brèves ", " théâtre pour les jeunes spectateurs ", " spectacles traditionnels du Japon », " poésie », " exposition », " débats », « tournées »), traduisant ainsi, sans hiérarchie, la diversité et l'éclatement d'une constellation de pratiques (figure 5). 
21 La programmation de la troisième saison (83-84), dans Le Journal de Chaillot qui succède à Chaillot et dont Georges Banu est également le rédacteur en chef, est présentée de façon bimestrielle, sur deux pages. La page de gauche propose une liste sobre, faite de titres et de dates, sans aucun regroupement complémentaire, ni par lieu ni par type de pratiques. Tandis que la page de droite présente un tableau, avec, pour chaque mois, une ligne par jour et quatre colonnes : « Jeunes spectateurs », " Grand Foyer », «Grand Théâtre ", "Théâtre Gémier ", la tarification correspondant aux distinctions des colonnes (un prix jeunes spectateurs, un prix grand foyer et un prix salles). Il ne semble plus nécessaire de mettre au premier plan la présence, dans la programmation, des marionnettes, des formes brèves et de la poésie. Que des spectacles relèvent ou non de catégories minoritaires et souvent infériorisées ne change rien à leur présentation. La diversité dans les faits existe mais elle n'est plus l'objet d'un affichage aussi insistant (figures $6 a$ et $6 b$ ).

22 Pour présenter la quatrième saison (84-85) les premiers numéros du Journal, dont JeanPierre Jourdain est devenu rédacteur en chef, conservent le principe de la liste des titres tout en réintroduisant une organisation surplombante par lieux, fortement atténuée néanmoins par le fait que le corps des titres des spectacles et des événements est nettement plus grand que celui des noms des lieux. Les regroupements par lieux disparaissent dans les numéros suivants de la saison, pour réapparaître dans les présentations des programmations des trois dernières saisons.

23 Ces observations sommaires, réalisées exclusivement à partir des journaux du théâtre ${ }^{21}$, montrent la double volonté d'ouvrir un établissement national à un large ensemble de pratiques et celle d'utiliser les lieux le plus possible, le tout dans une optique de décloisonnement, mais manifestent aussi la difficulté de présenter, avec les catégories habituelles, ce désordre voulu, cette diversité dans l'unité. Si de Faust ou de Tombeau pour cinq cent mille soldats en 1981 au Soulier de satin ou à Anacaona ${ }^{22}$ en 1988, le grand théâtre et le théâtre Gémier ont permis de mettre en scène des œuvres littéraires complexes et monumentales, le Grand Foyer de Chaillot a, quant à lui, été, de 1981 à 1988, un abri essentiel pour des formes en recherche qui souffraient d'un manque de lieux d'expression et d'une médiocrité de considération, notamment pour les arts de la marionnette.

Dans la chemise "Exposition de marionnettes» de la saison 1981-82 conservée aux Archives nationales se trouve un rapport de onze pages d'Agnès Van Molder (« ministre de la marionnette » pendant le mandat d'Antoine Vitez) intitulé « Réflexions sur les marionnettes depuis le mois de mai $1981^{23}$ ». Le document comprend des notes sur des spectacles présentés lors de festivals de marionnettes ou de façon isolée. Chaque spectacle répertorié est assorti d'un commentaire, de quelques mots à une dizaine de lignes, accompagné parfois de croquis. Une page "conclusions au 11 novembre 1981 » estime que la majorité de la cinquantaine de spectacles vus sur une période d'environ six mois est médiocre et suggère d'inviter le Centre national de Charleville-Mézières ${ }^{24}$ à proposer de dix à quinze spectacles qui seraient programmés dans un temps de la marionnette à Chaillot. Le rapport propose également de consacrer un mois par an le Théâtre Gémier aux enfants.

Certaines idées de Vitez et de "ses ministres » sont bien sûr restées au stade du rêve, soit parce que certaines commandes, incitations ou invitations n'ont pas été suivies d'effet (en particulier la plupart des commandes d'écritures contemporaines pour la marionnette), soit parce que des interdictions ont limité les possibilités d'utilisation des 
espaces pour des raisons de sécurité (notamment dans les escaliers et dans les espaces de circulation), mais un grand nombre d'idées ont néanmoins été mises en œuvre en sept saisons et le Chaillot des années 1981-1988 a véritablement été un lieu tout bruissant de voix ${ }^{25}$.

Édouard Glissant, dans les années 1990, a forgé le concept du Tout-monde pour parler de la mise en relation accélérée des peuples et des cultures et des inter-rétroactions qui en découlent et font qu'à l'intérieur de nous nous avons la totalité du monde. « Pour la première fois, les cultures humaines en leur semi-totalité sont entièrement et simultanément mises en contact et en effervescence de réaction les unes avec les autres $^{26} »$. De la forme brève à la forme longue, des écritures en français (français d'ici, français d'ailleurs) aux écritures en d'autres langues, du répertoire aux nouveautés, de l'adresse aux jeunes spectateurs à l'adresse aux spectateurs adultes, de la très grande jauge (plus de 1000 places) à la toute petite jauge (moins de 100 places), du répertoire classique aux écritures contemporaines, la cité utopique de Vitez à Chaillot me semble avoir été une cité du tout-théâtre en cela qu'elle a mis en relation une diversité de langages et, ce faisant, encouragé une forme de créolisation ${ }^{27}$ généralisée ${ }^{28}$.

Figure 1

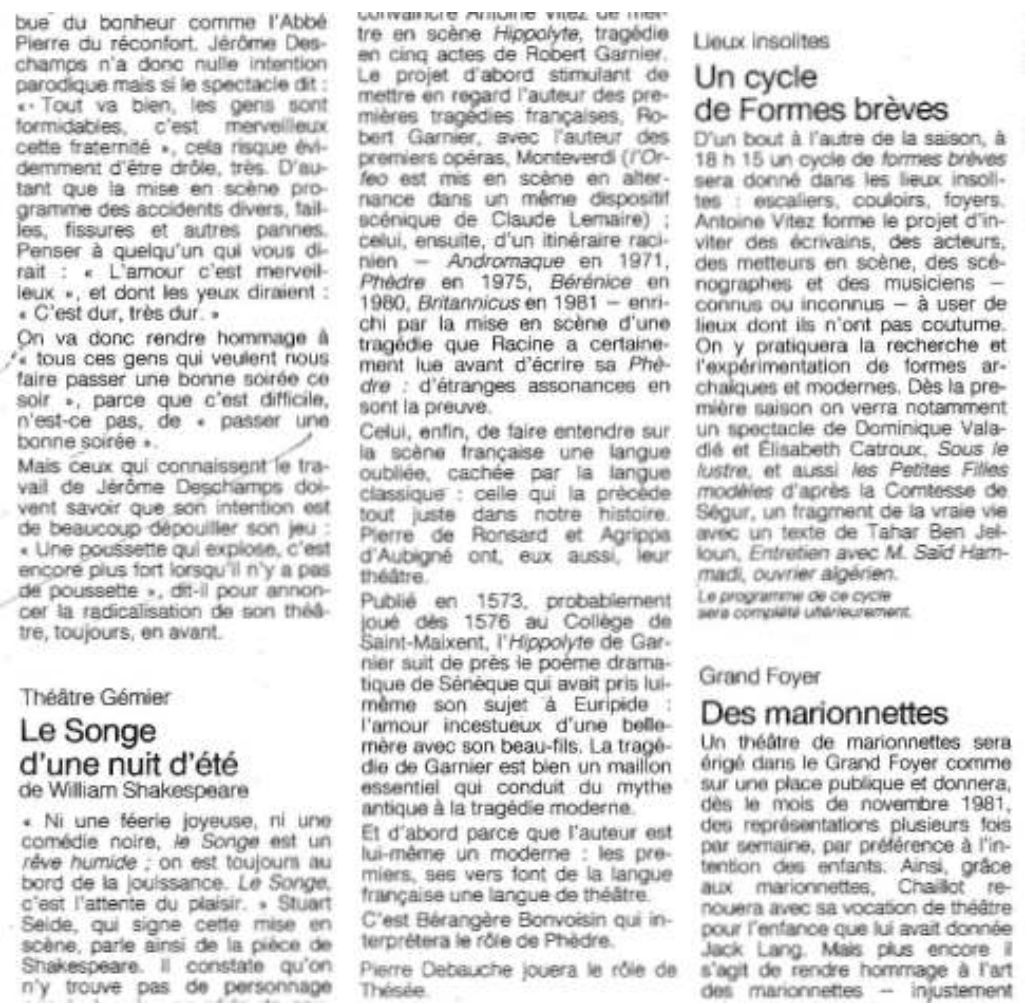

Chaillot, avant-numéro, mai 1981, p.3. Archives personnelles. 
Figure 2

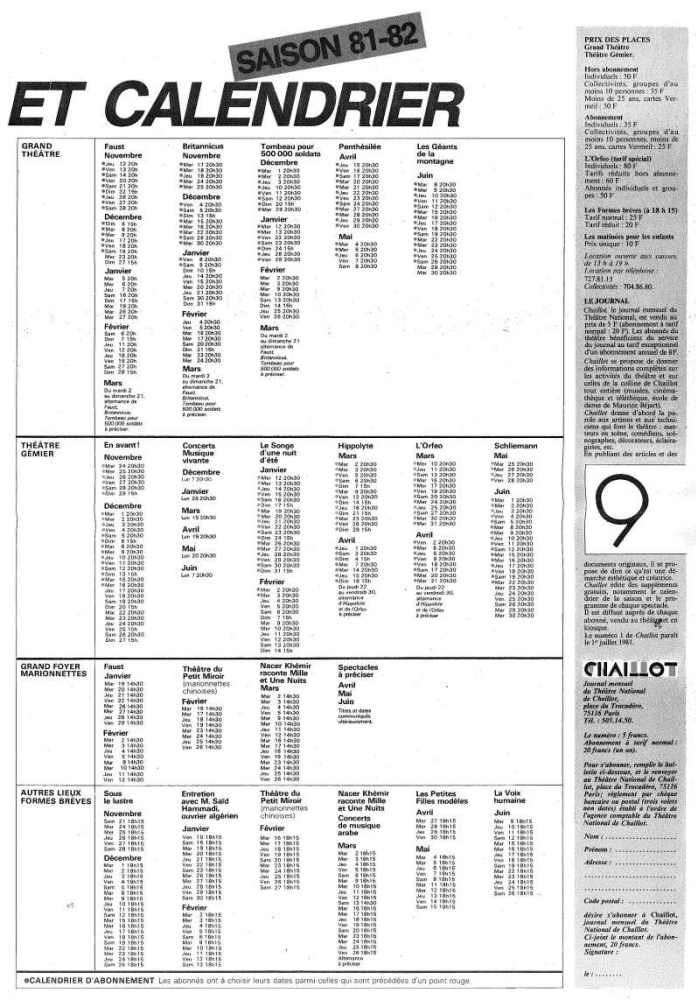

28 Chaillot, $\mathrm{n}^{\circ} 1$, juillet 1981, p. 9. Archives personnelles.

Figure 3

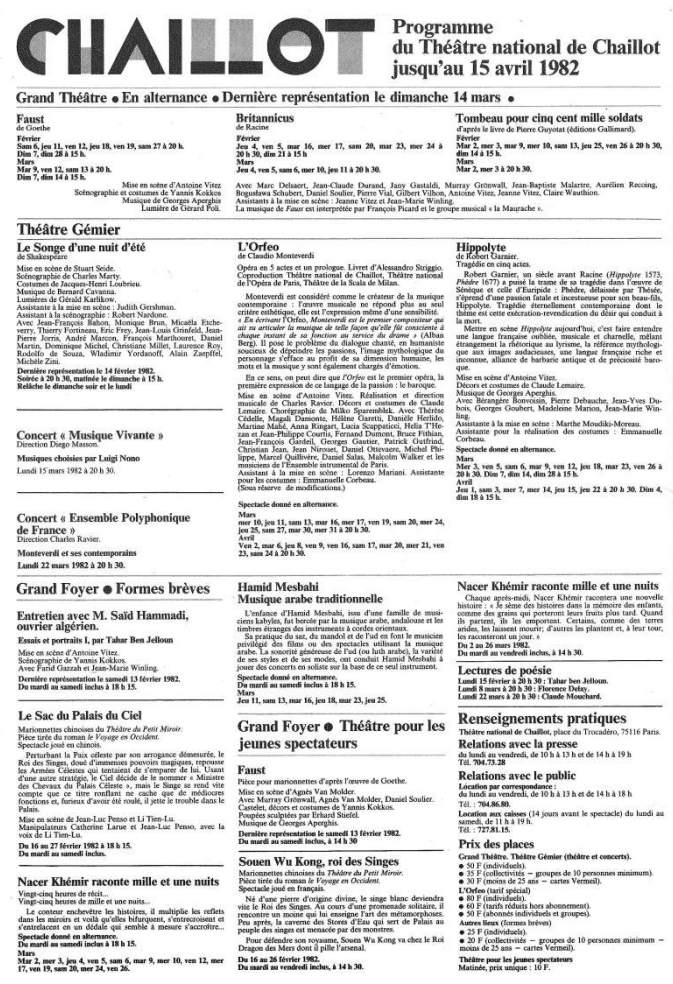

29 Chaillot, $\mathrm{n}^{\circ} 4$, avril 1982, p. 8. Archives personnelles. 
Figure 4

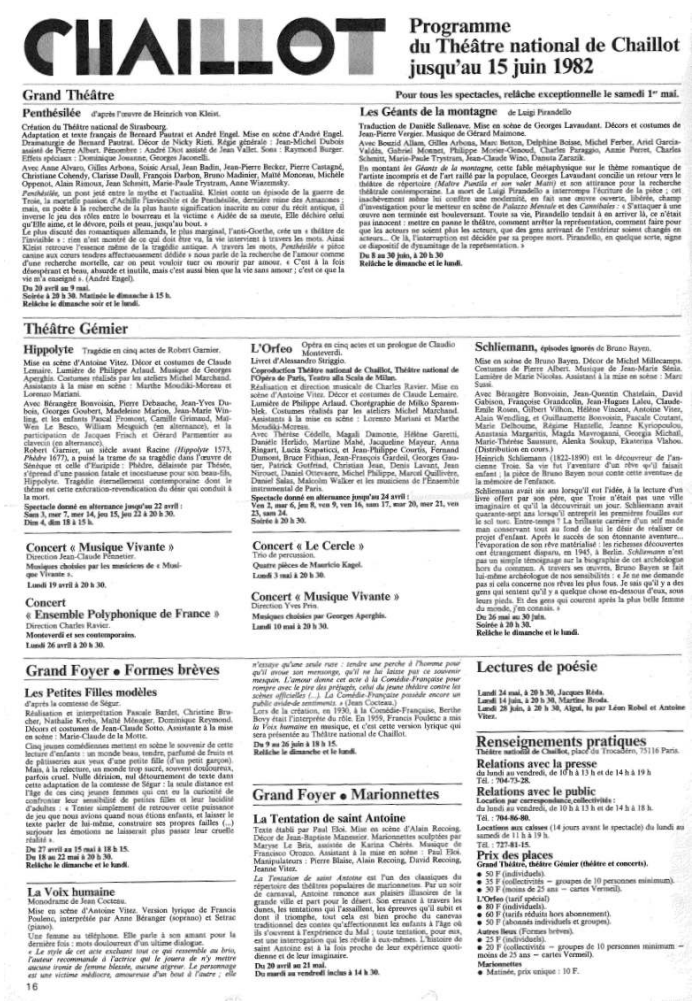

30 Chaillot, $\mathrm{n}^{\circ} 5$, avril 1982, p. 16. Archives personnelles.

Figure 5

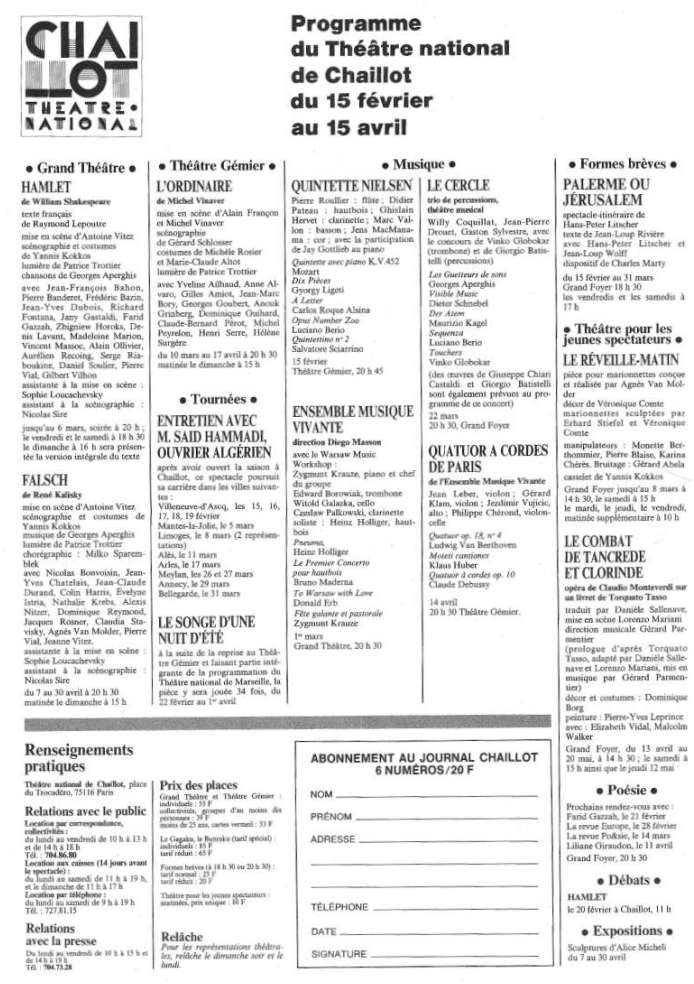


31

Chaillot, $\mathrm{n}^{\circ} 10$, février 1983, p. 16. Archives personnelles.

Figure $6(a, b)$
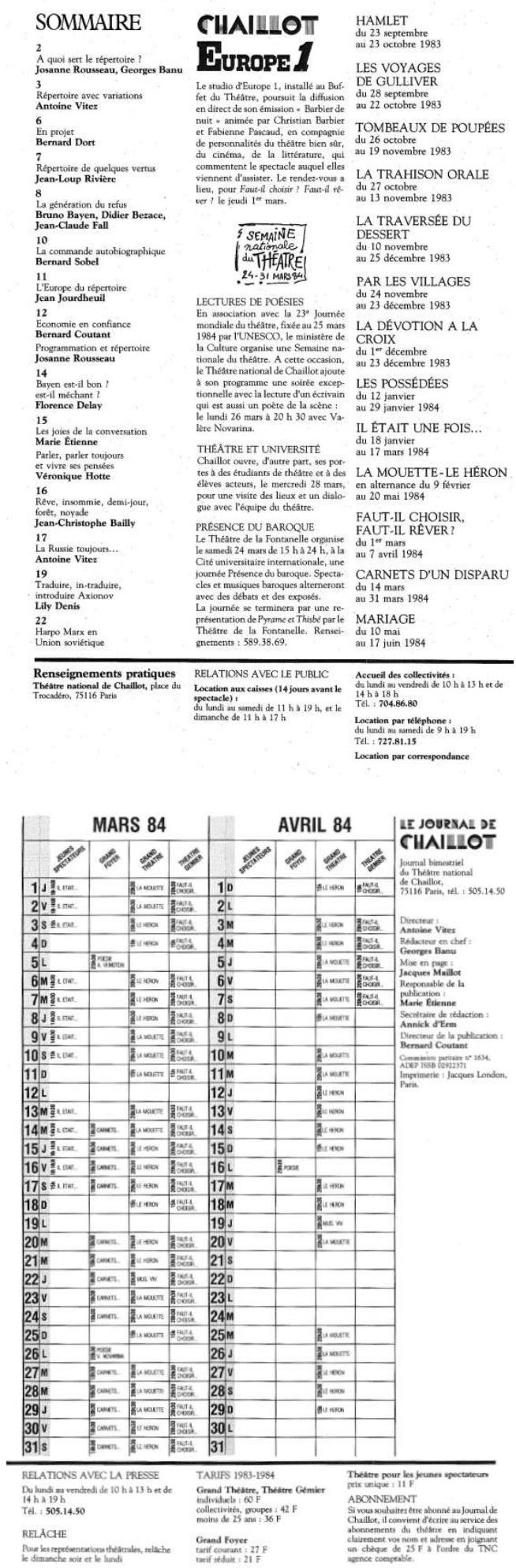

Le journal de Chaillot, $\mathrm{n}^{\circ} 16$, mars 1984, p. 26-27. Archives personnelles. 


\section{NOTES}

1. VITEZ (Antoine), «L'abri ou l'édifice», propos recueillis par Jean-Pierre Léonardini, L'Architecture d'aujourd'hui, n 199 : "Les lieux du spectacle », octobre 1978, réédité dans Antoine Vitez, Le Théâtre des idées, anthologie proposée par Danièle Sallenave et Georges Banu, Paris, Gallimard, collection « Pratiques du théâtre », [1991] 2015, par la suite désigné TDI, p. 91.

2. TDI, «L'abri ou l'édifice », p. 90.

3. J'ai mené plusieurs entretiens très éclairants avec Roland Monod, la plupart dans le cadre de mes travaux sur les relations théâtre/réel dans le théâtre français des années 1960 (au sujet de l'adaptation et de la mise en scène du Vicaire de Rolf Hochhut par Peter Brook et Georges Dabron, au sujet de l'écriture et de la mise en scène du Dossier Oppenheimer par Jean Vilar, au sujet des lectures et de sa mise en scène de Pour des raisons de cœur de Xavier-Agnan Pommeret). Les souvenirs dont je fais état ici m'ont été confiés dans le cadre d'une enquête que j'ai réalisée pendant l'été 2009 sur les liens d'Antoine Vitez avec Jean Vilar et avec les équipes engagées dans la politique de la décentralisation théâtrale.

4. Jean Vilar dirige le TNP de 1951 à 1963. La première saison est programmée à Suresnes. Après le déménagement de l'ONU, le TNP s'installe au Palais de Chaillot et y reste pendant la direction de Georges Wilson, de 1963 à 1972.

5. Jeune homme, Antoine Vitez est admiratif du travail d'acteur et de metteur en scène de son aîné auquel, par la suite, il se réfère souvent, mais, jamais, sur scène, il ne joue pour lui ni avec lui, jamais, non plus, il ne le dirige. Leurs premières relations professionnelles se font par le truchement de cette autre scène du théâtre que sont les revues. Théâtre populaire tout d'abord, qui, dans son numéro quatre (1953), publie le premier article d'Antoine Vitez sur le théâtre, "La méthode des actions physiques de Stanislavski ». Les Conversations avec Émile Copfermann, dont le texte a été relu par Antoine Vitez avant leur première édition (1981), raconte qu'un jour Vilar croisant Vitez dans l'immense édifice aurait écorché son nom en lui disant bonjour et que ce dernier lui aurait répondu "je suis un comédien que vous avez engagé ». Le commentaire d'Émile Copfermann, «Vilar l'a oublié » (Copfermann, 25), donne au récit $\mathrm{du}$ malentendu une coloration plus amère et piquante que ne le font les souvenirs que m'a confié Roland Monod. Dans Les Conversations, Vitez ne parle d'ailleurs que de façon très générale du travail artistique de Vilar, précisant qu'à partir de la mise en scène d'Arturo Ui de Brecht (1960), qui a marqué à ses yeux le « commencement d'un déclin » (Copfermann, 24), il a commencé à moins s'y intéresser, mais ne mentionnant pas l'admiration spontanément et unanimement mentionnée par ses proches lors des entretiens que j'ai réalisés en juin et juillet 2009. Voir JoInNAULt (Brigitte), "'Élitaire pour tous': oxymore ou pléonasme?", L'Annuaire théâtral $n^{\circ} 49$, printemps 2011, p. 77-92. https://www.erudit.org/fr/revues/annuaire/2011-n49annuaire0114/1009303ar/ consulté le 19 novembre 2018. Cette parenthèse ne vise pas à établir la supériorité d'une version sur une autre sur un point anecdotique au regard des parcours des deux hommes, mais à souligner l'importance de garder à l'esprit la fragilité et subjectivité inhérente au témoignage. Les archives du fonds Jean Vilar conservé par les Archives nationales nous permettraient peut-être d'établir avec plus de précision factuelle l'histoire de ce bref passage d'Antoine Vitez dans la troupe du TNP. Voir LACOUSSE (Magali), « Le fonds du Tnp, sous la direction Jean Vilar », La Gazette 
des Archives, $\mathrm{n}^{\circ}$ 229, 2013, p. 41-49, https://www.persee.fr/doc/ gazar_0016-5522_2013_num_229_1_5188 consulté le 19 novembre 2018.

6. En février 1973 Jacques Duhamel est remplacé par Maurice Druon lui-même remplacé par Alain Peyrefitte. Le Président Georges Pompidou meurt le 2 avril 1974, Alain Poher assure l'intérim jusqu'à l'élection de Valéry Giscard d'Estaing le 27 mai. Michel Guy, alors nommé secrétaire d'État à la Culture du premier gouvernement Jacques Chirac, redistribue immédiatement les directions de plusieurs théâtres.

7. VITEZ (Antoine), «Positions et propositions pour le Théâtre national de Chaillot », dans VITEZ (Antoine), Écrits sur le théâtre, $\mathrm{n}^{\circ}$ 5, anthologie de textes réunis par Nathalie Léger, Paris, P.O.L, 1998, p. 136-145, la désignation É5 sera utilisée pour les prochaines citations de ce volume.

8. É5, p. 138.

9. ÉTIENNE (Marie), En compagnie d'Antoine Vitez. 1977-1984, Paris, Hermann, 2017, p. 68-69

10. É5, p. 143-144.

11. Antoine Vitez, cité par Marie Étienne, op. cit., p. 71.

12. ÉTIENNE (Marie), op. cit.,p. 116-117. Les premiers numéros du journal du théâtre mentionnent «Conseillers du Directeur du Théâtre national de Chaillot: Georges Aperghis, Yannis Kokkos, Agnès Van Molder, Pierre Vial ». Voir par exemple Chaillot, n 1, juillet 1981, p. 16.

13. Par ordre alphabétique : Arlette Bonnard, Claude Engelbach, Gonzalo Estrada, Xavier-Agnan Pommeret, Alain Recoing et Salah Teskouk.

14. Les 4 Molière désigne la mise en scène de quatre pièces de Molière comme s'il s'agissait d'une tétralogie, L'École des femmes, Le Tartuffe, Don Juan, Le Misanthrope, répétées pendant la même période et jouées en alternance dans les mêmes lieux, avec une unique scénographie de Claude Lemaire et un même groupe d'acteurs. La création a eu lieu en juillet 1978 au festival d'Avignon.

15. Création en mai 1980 au Théâtre des Amandiers de Nanterre et donné au TQI en juin 1980.

16. La trilogie sur l'enfer désigne la mise en scène dans une scénographie unique avec une même équipe de trois textes Faust de Goethe, Britannicus de Racine, et Tombeau pour cinq cent mille soldats de Pierre Guyotat par lesquels s'est ouverte la saison 1981-82 à Chaillot.

17. Voir Dizier (Anna) et Jacques (René), Antoine Vitez: «Faust», "Britannicus », "Tombeau pour 500000 soldats ", Paris, Solin, 1982.

18. Le livre de Marie Étienne (op. cit.) décrit l'état du TNC en 1981.

19. Didier Deschamps et son équipe m'ont permis de réaliser au printemps 2017 une expérience de conduite d'entretiens déambulatoires in situ, à Chaillot, essentiellement dans les espaces hors salles, mais également parfois dans la grande salle, en quête des souvenirs sonores de proches du cercle exécutif et des ministres d'Antoine Vitez. Le centrage des entretiens sur la mémoire sensorielle a fait spontanément surgir des souvenirs olfactifs.

20. Dans les faits, au cours des sept saisons que nous avons examinées, les rendez-vous de poésie, comme les concerts (exception faite de la programmation du Bar Bleue attachée à un lieu spécifique), ont été nomades dans Chaillot, s'installant parfois en salle, parfois hors salle.

21. Les numéros 0 (mai 1981) à 27 (mars 1998) sont notamment conservés aux Archives nationales sous la cote 20170180/119.

22. À propos d'Anacaona on pourra consulter JoInNAUlt (Brigitte), «Vitez entre les langues. De Phèdre (1975) à Anacaona (1988) », Revue Sciences/Lettres [En ligne], 6 | 2019, mis en ligne le 10 décembre 2018. URL : http://journals.openedition.org/rsl/2547; DOI : https://doi.org/10.4000/ rsl.2547

23. Arch. nat., 20170180/121, "Exposition de marionnettes de janvier à février 1982 : note d'Agnès Van Molder sur les festivals de marionnettes, notes, correspondance (1981-1982)". Document consulté au Théâtre national de Chaillot avant son versement aux Archives nationales.

24. L'Institut international de la marionnette a été fondé en 1981 à Charleville Mézières. 
25. «Chaillot tout bruissant de voix » est le nom d'une séquence du site multimédia Entendre le théâtre qui permet d'entendre des extraits sonores de spectacles et de les localiser sur un plan du palais de Chaillot. Voir JoInNault (Brigitte), "Chaillot tout bruissant de voix », site Entendre le théâtre, un voyage sonore dans le théâtre français du XXe siècle, HUTHWOHL (Joël) et MERVANT-ROUX (Marie-Madeleine) [dir.], éditions multimédia de la BnF, mis en ligne en février 2020. URL: http://classes.bnf.fr/echo/chaillot/.

26. GLISSANT (Édouard), Le Cri du monde dans Le Traité du Tout-monde, Poétique IV, Paris, Gallimard, 1997, p. 23.

27. Je me réfère ici encore à Édouard Glissant lorsqu'il estime que le «fait » de la créolisation « est d'entretenir relation entre deux ou plusieurs 'zones' culturelles, convoquées en un lieu de rencontre, tout comme une langue créole joue à partir de 'zones' linguistiques différenciées, pour en tirer sa matière inédite ", op. cit., p. 25.

28. Sur les circulations entre les univers théâtraux fréquentés par Antoine Vitez l'on pourra se reporter à la conclusion de JoINNAUlT (Brigitte), Antoine Vitez: La mise en scène des textes non dramatiques. Théâtre-document, théâtre-récit, théâtre-musique, Montpellier et Paris, Entretemps et Max Milo, coll. « Champ théâtral », 2019, p. 347-376.

\section{RÉSUMÉS}

Lorsqu'en 1980 le gouvernement français propose à Antoine Vitez de réfléchir à un projet pour le théâtre de Chaillot, ce dernier, alors directeur du Théâtre des Quartiers d'Ivry, rêve de pouvoir déployer un travail théâtral de grande envergure et de profiter de l'immensité de l'édifice pour en faire un abri accueillant des formes d'expression qui souffraient alors d'un manque de considération et peinaient à trouver une écoute digne et honorable dans le paysage artistique français. Cet article donnera idée de la manière dont, entouré d'artistes partenaires, Marie Étienne pour la poésie, Agnès Van Molder pour les marionnettes, Georges Aperghis pour la musique, Yannis Kokkos pour les scénographies, il entreprit de faire du palais une cité du toutthéâtre vouée à l'écoute des langages et des artistes vivants.

\section{INDEX}

Mots-clés : Théâtre national de Chaillot, Palais de Chaillot, mémoire théâtrale, mémoire du spectateur, entretiens

\section{AUTEUR}

\section{BRIGITTE JOINNAULT}

Maîtresse de conférences, université Côte d'Azur, chercheuse du Centre Transdisciplinaire de la Littérature et des Arts Vivants (CTEL), associée à l'UMR THALIM, membre du projet ANR ECHO 